\title{
BEARING ESTIMATION IN THE PRESENCE OF SENSOR POSITIONING ERRORS
}

\begin{abstract}
A thesis submitted to the Faculty of science at the University of Edinburgh for the degree of Doctor of Philosophy
\end{abstract}

by

L.P.H.R.SEYMOUR, B.SC.

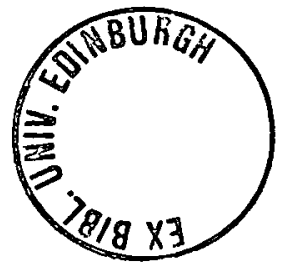




\section{DECLARATION OF ORIGINALITY}

This thesis, which was written entirely by me, describes research which I conducted on my own in the Department of Electrical Engineering at the University of Edinburgh.

L.P.H.K. Seymour

14 th November 1988 


\section{ACRNOWLEDGEMENTS}

I would like to thank Professor P.M.Grant and Dr.C.F.N.Cowan for supervising this project, particularly for their infinite patience during the writing of this thesis. I am also indebted to Dr.B.Mulgrew for his interest in the array calibration algorithm described in Chapter 4 and Mr.S.Mclaughlin for his advice on the statistical analysis presented in Chapter 3.

Finally, I would like to thank the science and Engineering Research Council and Racal Communications Equipment Limited for funding this work. 


\section{CONTENTS}

Title Page i

Abstract ii

Declaration of Originality ii

Acknowledgements iv

Contents $\quad v$

CHAPTER 1 INTRODUCTION 1

1.1 Bearing Estimation using a Phased Array 1

1.2 Research Topics in Bearing Estimation 7

1.3 scope of Thesis 9

1.4 Layout of Thesis 12

CHAPTER 2 BEARING ESTIMATION TECHNIQUES

2.1 Introduction 14

2.2 Sensor Positions 15

2.3 Received Signals 18

$\begin{array}{ll}2.4 \text { Signal Vectors } & 20\end{array}$

2.5 The Data Model 24

2.6 Properties of the Signal Vectors 27 
2.7 Covariance Matrices 28

2.8 Properties of the Noise Covariance Matrix 30

2.9 Properties of the Signal Covariance Matrix 31

2.10 Properties of the matrix product $\underset{\sim}{\mathrm{H}} \underset{\sim}{\underset{\sim}{H}} \underset{\sim}{H}$

2.11 Properties of the Covariance Matrix 35

2.12 Signal and Noise Subspaces 40

2.13 The MUSIC Algorithm $4 \mathbf{4 2}$

2.14 Conventional Beamforming $\quad 47$

2.15 The Minimum Energy Method 50

2.16 Computer simulation of Bearing Estimation 56 Techniques

2.17 summary

CHAPTER 3 THE EFFECTS OF SENSOR POSITIONING ERRORS

3.1 Introduction $\quad 69$

3.2 Modelling of Sensor Positioning Errors 73

3.3 Parameters of the Bearing spectrum 80

3.4 Computer Simulation 83

3.5 A Geometric View of Bearing Estimation 89

3.6 The Mean of the Bearing Error 100

3.7 The Variance of the Bearing Error 112

3.8 A General Quadratic Form for the spectrum 130 Value at the True Bearing

3.9 The Quadratic Form for Conventional 134 Beamforming 
3.10 The Quadratic Form for Minimum Energy

3.11 The Quadratic Form for MUSIC

3.12 The Mean of the Cosine of the Phase Error

3.13 The Mean of the General Quadratic Form

3.14 The Mean of the spectrum Value at the True 150 Bearing

3.15 The Variance of the General Quadratic Form 154

3.16 The Variance of the spectrum Value at the 160 True Bearing

3.17. The Mean and Variance of the Peak Height

4.1 Introduction 170

4.2 Array Calibration and Bearing Estimation 172

4.3 Sources of Error in Array Calibration 176

4.4 An Iterative Approach to Array Calibration 179

4.5 The Array Calibration Algorithm 190

4.6 Computer Simulation of the Array Calibration 198 Algorithm

4.7 summary

CHAPTER 5 CONCLUSIONS 
5.2 Specific Achievements

$\begin{array}{ll}5.3 \text { Limitations } & 226\end{array}$

5.4 Suggestions for Further Research

MUSIC - Multiple Signal Classification

\begin{tabular}{|c|c|}
\hline $\mathbf{N}$ & - number of sensors in array \\
\hline M & - number of signals being received \\
\hline$\vec{u}_{n}$ & - intended position of $n^{\text {th }}$ sensor \\
\hline$\stackrel{\sim}{n}_{n}$ & - true positions of $n^{t h}$ sensor \\
\hline$\Delta u_{n}$ & - error in position of $n^{\text {th }}$ sensor \\
\hline$\theta_{\mathrm{m}}$ & - bearing of $m^{\text {th }}$ signal \\
\hline $\mathbf{z}_{\mathrm{m}}$ & - unit vector pointing at $\mathrm{m}^{\text {th }}$ transmitter \\
\hline$\hat{\mathrm{q}}(\theta)$ & - signal vector for bearing $\theta$ \\
\hline$\underset{\sim}{\mathbf{R}}$ & - covariance matrix \\
\hline$\lambda_{n}$ & - nth eigenvalue of covariance matrix \\
\hline in & - $n^{\text {th }}$ eigenvector of covariance matrix \\
\hline
\end{tabular}




\section{CHAPTER 1 INTRODUCTION}

\subsection{Bearing Estimation using a Phased Array}

There are many applications for systems that can determine the direction from which a signal is being received, particularly in the fields of radio [1]-[7] and sonar [1], [8], [9]. If the bearing is measured from different locations, the position of the transmitter can be estimated using triangulation.

The task of finding the bearing, and possibly power, of a received signal is known as bearing estimation or direction finding. The simplest technique is to use a single highly directional sensor which is rotated mechanically to explore different bearings.

However, an alternative approach, and the one of interest here, is to use an array of several sensors, which are often arranged in a straight line or around the circumference of a circle [10]. If the distances between the sensors are comparable with the wavelength of the signal being received, the bearing can be computed from the phase shifts between the signals from different sensors. The work reported in this thesis concerns the 
effects of errors in the sensor positions on this process.

The basic principle of bearing estimation is illustrated in Figure 1.1, which shows an array of five sensors in a straight line with a spacing of half a wavelength between adjacent sensors. The array is receiving a single sinusoidal plane wave transmission with a bearing of approximately 13 degrees and the diagram shows the wavefronts (peaks) passing over the sensors.

The wavefronts arrive at sensor 1 first then pass over each of the other sensors in turn and the output signals from the sensors are therefore time-delayed, or phase-shifted, versions of each other. Since the phase angle at the transmitter is unknown, the signal from sensor 1 is arbitrarily chosen to act as a reference and the phases at the other sensors are measured with respect to sensor 1 as illustrated.

It should be clear that the phase shifts depend on the bearing of the signal. In particular, if the wavefronts were parallel with the line of the array, the sensor outputs would all be identical and there would be no phase differences.

In the example illustrated, it would be straightforward to work backwards from the measured phase angles to the 
3

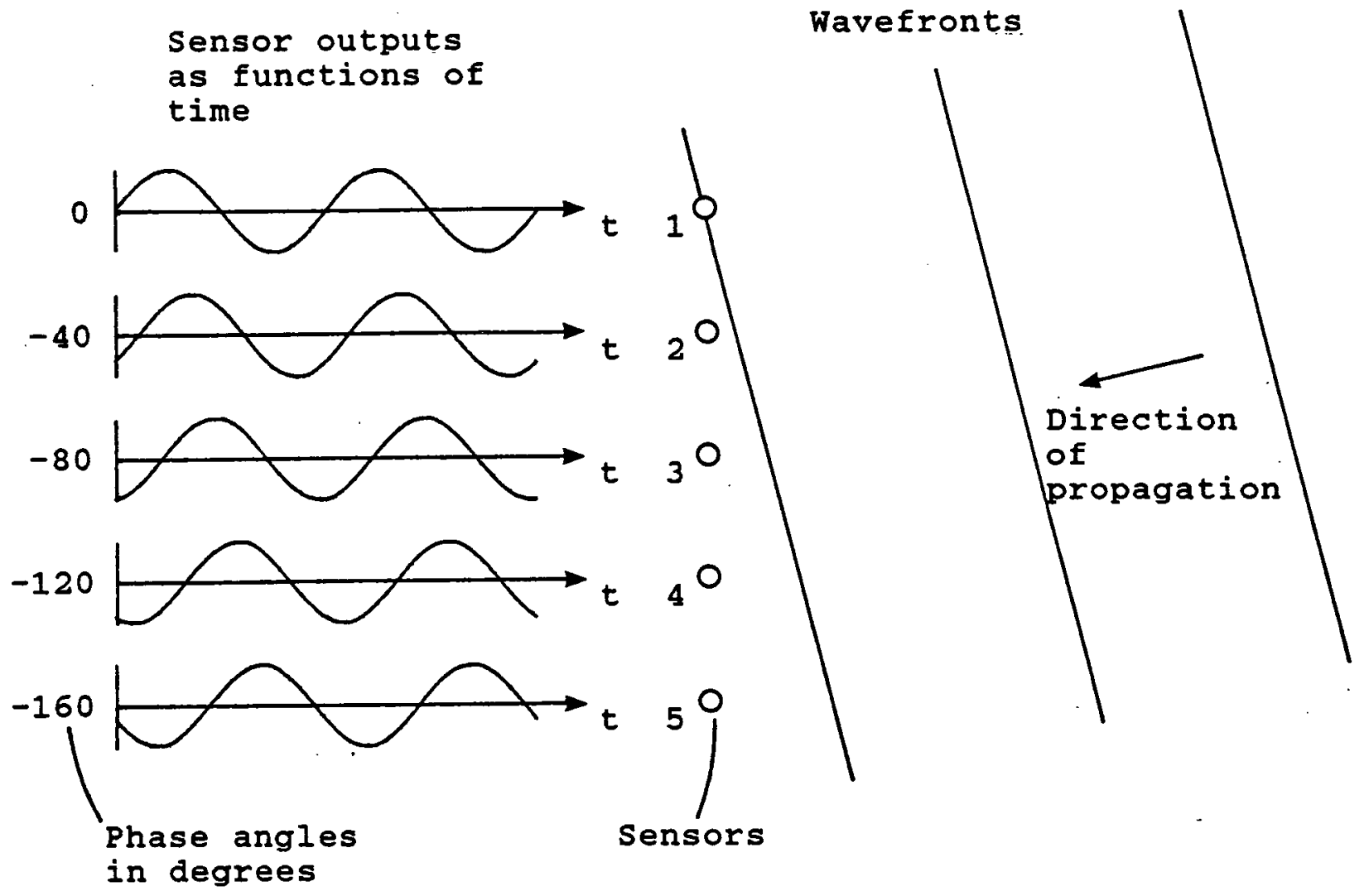

Figure 1.1 Sensor array 
bearing of the signal using trigonometry. In practice, however, the following factors tend to complicate the situation.

In view of the plane wave assumption, individual cycles of the waveform are indistinguishable and the observed phase angles are all folded into the range $-\pi$ to $\pi$ radians. For example, $5 \pi / 2$ would be measured as $\pi / 2$, any complete cycles of $2 \pi$ radians being lost. It follows that the phase difference observed between a particular pair of sensors can often be explained by bearings other than the true one. Bearing estimation algorithms resolve these ambiguities by combining the information from all of the pairs of sensors in the array.

Another problem is noise, which may be received with the signal or generated in the receivers themselves. Noise corrupts the measured phase angles, causing errors in the bearing estimation. It is common practice to record a large number of samples from each sensor then apply an averaging process in an attempt to reduce the effects of noise.

Further complications arise when there is more than one signal present. In this case, the output from each sensor is a sum of sinusoids with different phase angles. Even in situations where only one signal is expected, the 
desired signal may be accompanied by reflections from the atmosphere or the surrounding terrain, producing an effect known as multipath [11]-[15]. It is very difficult to separate the direct and reflected signals if there is a constant phase relationship between them and this is a severe practical problem in many applications.

Bearing estimation is essentially a form of spectral analysis which operates in a spatial domain rather than the more common frequency domain [16]. In time series analysis, the aim is to produce a spectrum of power against frequency. Similarly, bearing estimation algorithms generate a spectrum of power against bearing. Peaks in the spectrum are assumed to indicate signals which are being received, as illustrated in Figure 1.2.

Most bearing estimation techniques are closely related to algorithms used for time series analysis, and methods such as the Fourier transform [17]-[19], autoregressive analysis [17]-[20], the minimum energy method [17], [18], [21], and eigenvector methods [11], [17], [18], [22], [23] have all been adapted for use in bearing estimation [16] . 


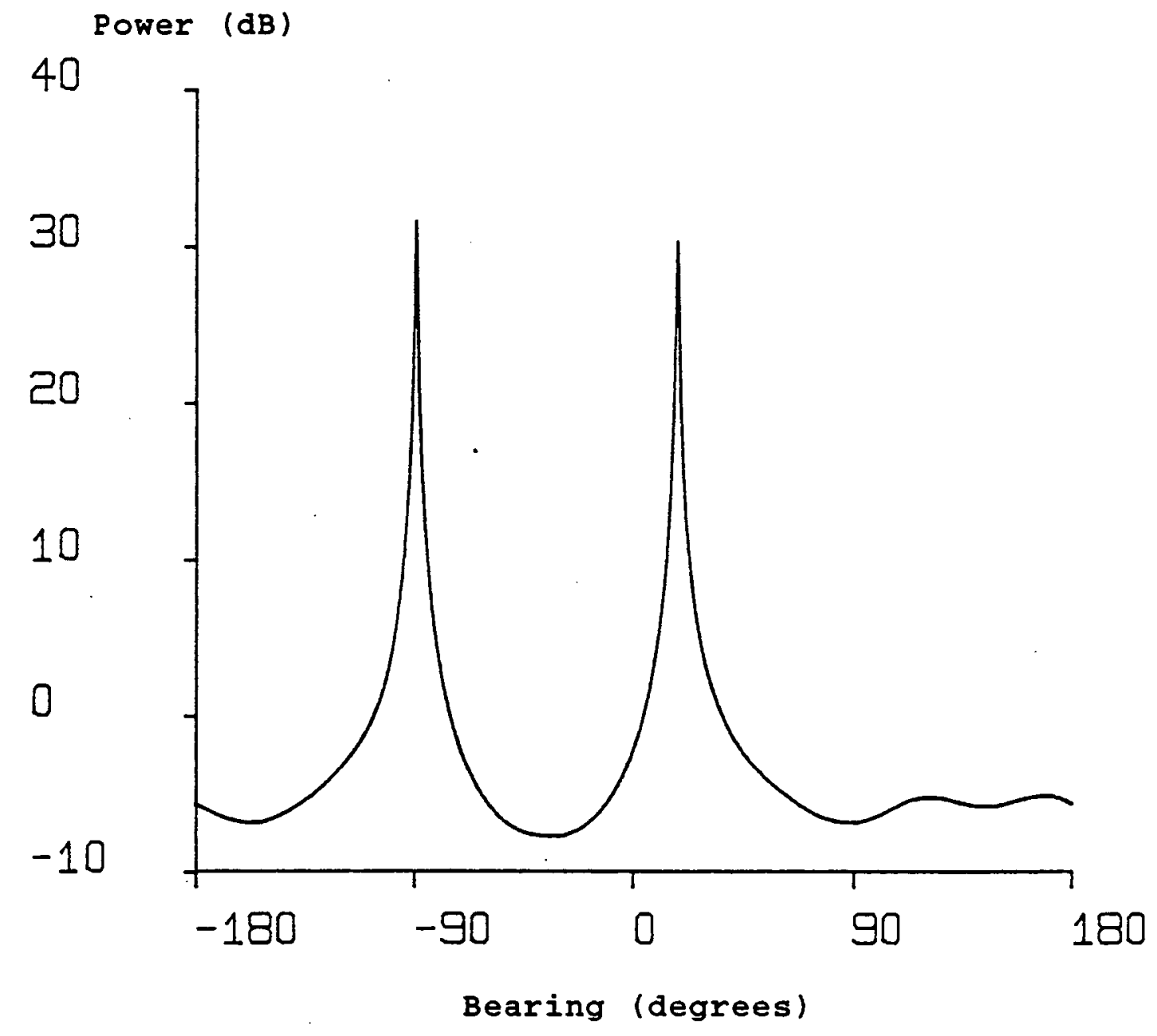

Figure 1.2 Bearing spectrum 


\subsection{Research Topics in Bearing Estimation}

In view of the close relationship between bearing estimation and time series analysis, it is not surprising that research topics in the two fields tend to be fairly similar. In both areas, there are a number of algorithms which perform the same task with different compromises between performance, computational cost, and robustness. Researchers have devoted themselves to understanding the basic properties of the algorithms, comparing the techniques with each other, and modifiying them so as to cope better with practical problems such as high noise levels.

Some material has been published clarifying the link between bearing estimation and time series analysis and presenting a common methodology for the various techniques [16]. The algorithms most commonly discussed are conventional beamforming [12], [16], [24]-[27], minimum energy (sometimes referred to as maximum likelihood) [12], [16], [24]-[26], [28]-[34], autoregressive analysis (linear prediction) [12], [16], [24], [28], [35], [36], eigenvector methods (particularly MUSIC) [11], [16], [22], $[25]-[27],[29],[37]-[43]$, and maximum entropy [16] , [28]- [32]. 
One of the most important properties of a bearing estimation algorithm is its resolution, which is the ability of the technique to distinguish two signals with similar bearings and produce two distinct peaks in the bearing spectrum. A number of authors have compared the resolution properties of different techniques [12], $[24]-[27],[29],[30],[37],[44]$. Some researchers have produced super-resolution algorithms which are claimed to have exceptionally good resolution properties [30], [37], $[45]$.

Some effort has been devoted to improving the performance of the algorithms at poor signal to noise ratios [45]-[48] and a great deal of research has been aimed at developing algorithms which cope with the highly correlated signals encountered when multipath is present [11], [13-15], [49]-[59]. There has also been work on the synthesis of optimal array geometries [60]-[62] and the development of bearing estimation algorithms with low computational requirements [63]-[65]. Some authors have described completely new algorithms, such as ESPRIT [66], [67].

One area which appears to have received little attention is the effect of errors in the positions of the sensors. Since the phase difference between two sensors is determined by both the bearing of the signal and positions of the sensors, errors in the positions cause errors in 
the phase measurement, which in turn lead to errors in the bearing spectrum.

The study of sensor positioning errors was selected as the main area for the research described in this thesis and the precise aims of this work are discussed in the next section. While the thesis was being prepared, a number of references on the same topic as Chapter 4 were discovered [49], [68]-[81]. Indeed, the subject appears to be becoming quite popular as evidenced by the number of papers on array calibration algorithms presented at the International Conference on Acoustics, speech, and signal Processing in 1988 [49], [68]-[70].

\subsection{Scope of Thesis}

The work presented in this thesis concerns the sensitivity of phased array bearing estimation systems to errors in the positions of the sensors. The research is specifically aimed at portable radio direction finding systems operating at narrow aperture in the HF frequency band (i.e. $3 \mathrm{MHz}$ to $30 \mathrm{MHz}$ ). The main application for such systems is the interception of radio transmissions in a battlefield environment. It is assumed that the transmitters are within approximately 60 miles of the 
direction finding array, meaning that propagation is entirely by ground wave [82], [83] and an azimuth-only system is sufficient.

Within the HF band, frequencies of between $6 \mathrm{MHz}$ and $10 \mathrm{MHz}$ are particularly common, resulting in wavelengths of between 30 and 50 metres. At these frequencies, a direction finding array with a spacing of, say, half a wavelength between adjacent sensors is clearly too large for all of the sensors to be mounted on a common structure. The result is that the sensors must be positioned individually and small errors in the positions of the sensors are likely to occur.

The research is divided into two main sections, the first of which aims to produce theoretical expressions relating factors such as the average error in an estimated bearing to the tolerance on the sensor positions and this is repeated for various bearing estimation algorithms. The second objective is to develop a calibration procedure to improve the performance of the algorithms when sensor positioning errors are present.

The analysis of the effects of the errors is approached by considering the $X$ and $Y$ coordinates of the sensor positions to be normally-distributed random variables. The mean of each variable is the correct value of the 
corresponding coordinate, while the (very small) variance expresses the magnitude of the errors likely to be produced when setting up the array.

Having made this definition, all of the quantities which are influenced by the sensor positions become random variables with means and variances determined by the variance of the sensor positions. Thus the analysis proceeds by observing the way in which this variance propagates through the bearing estimation algorithms.

The algebra required to do this is not straightforward and a number of approximations are required to make the problem manageable. However, computer simulations confirm the theoretical results over a range of variances.

The second piece of work develops a calibration algorithm to compensate for sensor positioning errors. The algorithm detects and measures errors in the sensor positions by analyzing the sensor outputs while signals with known bearings are being received. Clearly, this limits the application of the method to situations where it is practical to provide such signals.

The algorithm uses the idea of triangulation in a novel way. Rather than using two or more direction finding arrays to locate the position of a single transmitter, two 
or more transmitters are used to estimate the positions of the sensors in a single direction finding array. Once the true sensor positions have been discovered in this way, they can be taken into account subsequently when performing bearing estimation on signals with unknown bearings.

\subsection{Layout of Thesis}

Chapter 2 presents a detailed mathematical description of a number of bearing estimation techniques, the aim being to establish the concepts and notation required for the original work described in the later chapters. The discussion concentrates on the MUSIC algorithm [22], although the conventional beamforming and minimum energy methods are also described. The three techniques are compared using computer simulations.

The original research analyzing the effects of sensor positioning errors is described in Chapter 3. A statistical model is defined in which the errors are characterized by the variance of the sensor positions. Equations are derived relating high-level parameters of the bearing spectrum such as the means and variances of the peak height and peak position to the variance in the 
error model. This is repeated for different bearing estimation techniques and the results are confirmed using computer simulations.

Chapter 4 presents the original work on the array calibration algorithm. Using concepts from the MUSIC technique described in Chapter 2 , a cost function is defined which expresses the error between the (unknown) true sensor positions and the ones assumed in the bearing estimation process. The calibration algorithm attempts to discover the true positions by iteratively updating a set of estimated positions in a way which minimizes the cost function. Computer simulations confirm the effectiveness of the approach.

Chapter 5 presents conclusions on the material which has been covered. A number of suggestions for further research are included. 


\section{CHAPTER 2 BEARING ESTIMATION TECHNIQUES}

\subsection{Introduction}

This chapter provides the background material for the original research presented in Chapters 3 and 4 . The account is based on the definitive papers published by Johnson [16] and schmidt [11], [22] .

The chapter is broadly divided into three parts, the first of which develops a data model to describe the samples, or snapshots, obtained from an array of sensors. The discussion starts by introducing notation for the positions of the sensors, the bearings and frequencies of the signals being received, and the sampling rate and then an expression is derived for the outputs from the sensors as functions of time. This is expressed in matrix notation and a number of algebraic properties are highlighted.

The second part of the chapter considers the problem of bearing estimation, which involves recovering the bearings of the signals from the sequence of samples implied by the data model. The covariance matrix of the sensor outputs is introduced and its algebraic properties are considered 
in some detail. In particular, an analysis of the eigenvalues and eigenvectors of the covariance matrix leads to a discussion of signal and noise subspaces and a derivation of the MUSIC technique for bearing estimation. The conventional beamforming and minimum energy methods are also described.

The third part of the chapter presents a number of bearing spectra produced by a computer simulation system, the aim being to introduce the simulation scenario used in Chapters 3 and 4 . The results in this chapter merely confirm well-known basic properties of the MUSIC, conventional beamforming, and minimum energy methods.

\subsection{Sensor Positions}

In Figure 2.1, an array of $\mathrm{N}$ omni-directional sensors is receiving signals from different directions. The index $n$ always lies in the range 1 to $N$ and can thus be used to refer to any sensor in the array.

The processing of the sensor outputs is assumed to be narrowband with a nominal operating frequency of $\omega_{c}$ radians per second and the sensor positions are specified using a Cartesian coordinate scheme in which the units are 


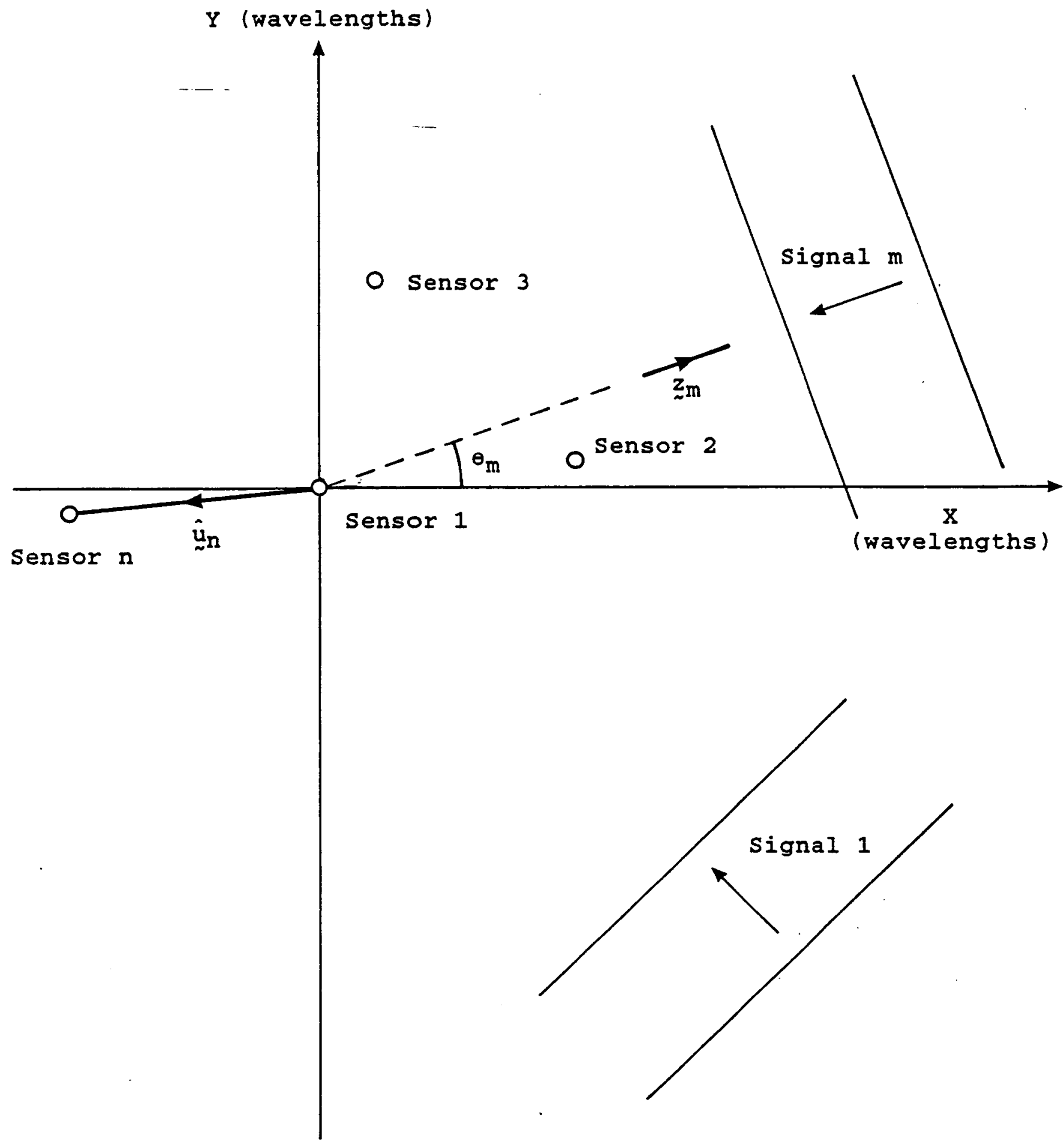

Figure 2.1 General bearing estimation problem 
wavelengths at this frequency. The use of this convention rather than units of distance provides convenient normalization and accommodates the fact that the apparent array size depends on frequency.

All of the sensors lie in the same horizontal plane and the position of sensor $n$ within this plane is described by the vector $\hat{u}_{n}$, which expresses the position relative to the origin. The origin is defined to be the position of sensor 1 .

$$
\hat{\mathrm{u}}_{1}=\underline{\sim}
$$

The vector $\hat{u}_{n}$ actually describes the intended position of sensor $n$, which may be slightly different from its true position when the array is set up. Sensor positioning errors are the subject of the original research described in Chapters 3 and 4. This chapter, however, follows the normal convention of assuming that there are no sensor positioning errors present. Under this assumption, the true sensor positions are equal to the intended ones and are therefore known precisely. 


\subsection{Received Signals}

Returning to Figure 2.1, the array of $N$ sensors is receiving a total of $M$ signals. Since the array can resolve at most $N$ - 1 signals [8], it is assumed that the number of signals is less than the number of sensors.

$$
M<N
$$

The signal index $m$ lies in the range 1 to $M$. All of the received signals are plane waves (i.e. sinusoids) and it is assumed that the transmitters are sufficiently distant for the wavefronts to be considered straight and parallel over the area covered by the array. The bearing of signal $\mathrm{m}$ is therefore the same from each sensor and is

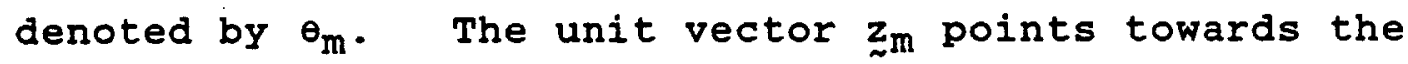
transmitter and the direction of propagation is therefore $-z_{m} \cdot$

Although the data model involves the bearing $\theta_{m}$, it does not include the distance from the array to the transmitter. Instead, the state of the transmitter is described by a phasor $a_{m}(k)$, which represents the signal that would be observed at the origin of the sensor coordinate system. $\quad k$ is the sample index, which ranges from 1 to $k$ and $a_{m}(k)$ is defined as follows. 


$$
a_{m}(k)=A_{m} \exp \left\{j\left(2 \pi(k-1) \frac{\omega_{m}}{\omega_{s}}+\alpha_{m}\right)\right\}
$$

$A_{m}, \omega_{m}$, and $\alpha_{m}$ are the amplitude, frequency and phase angle of signal $\mathrm{m}, \omega_{\mathrm{s}}$ is the sampling frequency, and $j$ is the square root of -1 . Since the system is narrowband, all of the signals should ideally have the same frequency $\omega_{c}$. However, sinusoids with identical frequencies would be mutually correlated since the same phase relationships would persist throughout the sampling period. In practice, received signals usually drift with respect to each other over a period of time and this phenomenon can be modelled by allowing each signal to have a slightly different frequency.

$$
\omega_{1} \simeq \omega_{2} \simeq \ldots \simeq \omega_{M} \simeq \omega_{C}
$$

The bearing estimation techniques described in this chapter assume that the signals are uncorrelated. Sets of two or more mutually correlated signals are encountered in practice when problems such as multipath are present and considerable research effort has been directed towards modifying the techniques so as to cope with this situation [11], [13]-[15], [50], [51]. The problem will not, however, be addressed here. 
Since the sensor positions are specified in terms of the ideal operating frequency $\omega_{C}$, the array size is distorted slightly for signals at other frequencies. It is assumed, however, that this effect is too small to be significant and that the apparent shifts in the sensor positions are much smaller than those caused by genuine sensor positioning errors.

\section{$\underline{2.4 \text { Signal Vectors }}$}

So far, the discussion has shown that the signal observed at the origin due to transmitter $m$ can be represented by the phasor $a_{m}(k)$. Since the wavefronts generally arrive at a particular sensor some time before or after passing over the origin, the signals observed by the sensors are time-shifted versions of $a_{m}(k)$. In view of the plane wave assumption, individual cycles of $a_{m}(k)$ cannot be distinguished and the time delays can therefore be expressed as phase shifts.

In Figure 2.2, $\hat{\epsilon}_{\mathrm{nm}}$ is the distance from the origin to sensor $n$ along the axis of propagation of signal $m$ and it is this distance which determines the phase shift between the origin and the sensor. Stated mathematically, $\hat{\epsilon}_{\mathrm{nm}}$ is the length of the component of the sensor position $\hat{u}_{n}$ 


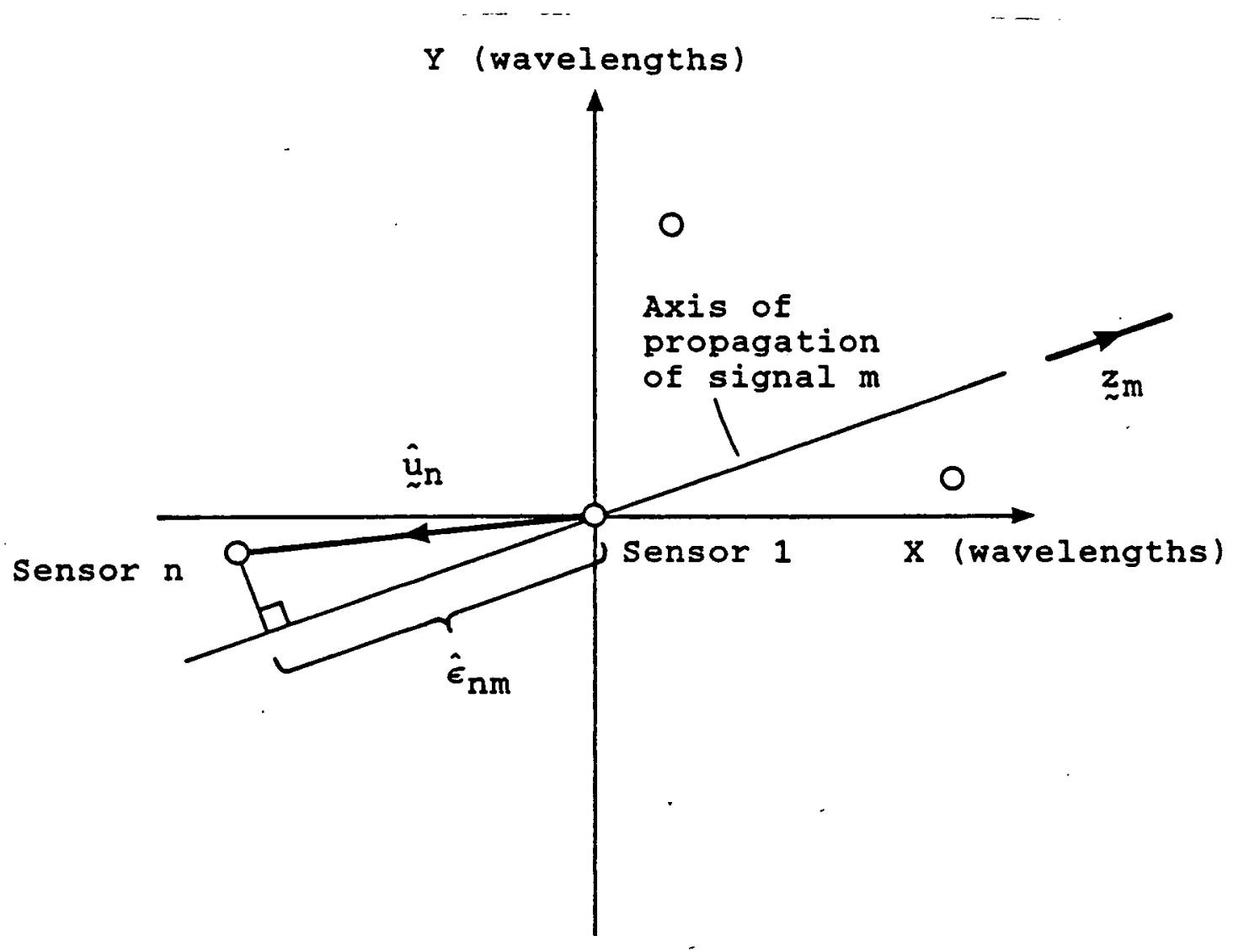

Figure 2.2 Distances along axis of propagation 
along the bearing of the transmitter $z_{\sim} m$ and can be calculated as the scalar product of the two vectors.

$$
\hat{\epsilon}_{\mathrm{nm}}=\underline{\sim}_{\mathrm{n}} \cdot \mathbf{z}_{\mathrm{m}}
$$

The phase shift from the origin to the position of sensor $\mathrm{n}$ is $\hat{\Psi}_{\mathrm{nm}}$ radians. Since the distance $\hat{\epsilon}_{\mathrm{nm}}$ is measured in wavelengths, it can be converted to a phase angle by multiplying by $2 \pi$.

$$
\hat{\Psi}_{\mathrm{nm}}=2 \pi \hat{\epsilon}_{\mathrm{nm}}
$$

The phasor describing the signal observed by sensor $\mathrm{n}$ is denoted by $s_{n m}(k)$ and is equal to $a_{m}(k)$ with a phase shift of $\hat{\Psi}_{\mathrm{nm}}$ applied. An expression for $s_{n m}(k)$ is found by modifying (2.3) to include the new phase term.

$$
\begin{aligned}
s_{n m}(k) & =A_{m} \exp \left\{j\left(2 \pi(k-1) \frac{\omega_{m}}{\omega_{s}}+\alpha_{m}+\hat{\psi}_{n m}\right)\right\} \\
& =\exp \left(j \hat{\psi}_{n m}\right) A_{m} \exp \left\{j\left(2 \pi(k-1) \frac{\omega_{m}}{\omega_{s}}+\alpha_{m}\right)\right\} \\
& =\hat{q}_{n m} a_{m}(k)
\end{aligned}
$$

where

$$
\hat{\mathrm{q}}_{\mathrm{nm}}=\exp \left\{j \hat{\psi}_{\mathrm{nm}}\right\}
$$


The phasor $\hat{q}_{n m}$ is a complex number with unit magnitude and an argument equal to $\hat{\psi}_{n m}$. It should be noted that $\hat{q}_{n m}$ contains less information than $\hat{\psi}_{\mathrm{nm}}$ since any complete cycles of $2 \pi$ radians in $\hat{\psi}_{n m}$ are lost in the process of evaluating $(2.8)$.

Since sensor 1 is always positioned at the origin, the signal observed by this sensor is $a_{m}(k)$ without any phase shift and the following rules apply to sensor 1 for any signal $\mathrm{m}$.

$$
\begin{aligned}
& \hat{\epsilon}_{1 \mathrm{~m}}=0 \\
& \hat{\psi}_{1 \mathrm{~m}}=0 \\
& \hat{q}_{1 \mathrm{~m}}=1 \\
& s_{1 \mathrm{~m}}(k)=a_{m}(k)
\end{aligned}
$$

Equations (2.8), (2.11), and (2.12) can be expressed for all $\mathrm{N}$ sensors using the following matrix notation.

$$
\mathbf{s}_{\mathfrak{m}}(k)=\hat{\mathfrak{q}}_{m} a_{m}(k)
$$

where

$$
\begin{aligned}
& \mathbf{s}_{m}(k)=\left[\begin{array}{llllll}
a_{m}(k) & s_{2 m}(k) & s_{3 m}(k) & \ldots & s_{N m}(k)
\end{array}\right]^{T} \\
& \hat{q}_{m}=\left[\begin{array}{lllll}
1 & \hat{q}_{2 m} & \hat{q}_{3 m} & \ldots & \hat{q}_{N m}
\end{array}\right]^{T}
\end{aligned}
$$


The column vector $\hat{\mathrm{q}}_{\mathrm{m}}$ is known as the signal vector for signal $\mathrm{m}$ and this vector completely characterizes the response of the array to a signal from the direction $\mathbf{z}_{m}$. If two different bearings produce identical signal vectors then transmissions from those directions cannot be distinguished. This is illustrated in Figure 2.3 using a Iinear array, which is a common configuration in which the sensors are placed in a straight line with equal spacing between adjacent sensors. An array of this type produces identical signal vectors for the bearings $\theta_{m}$ and $\pi-\theta_{m}$.

\subsection{The Data Model}

The vector $s_{m}(k)$ only accounts for the contribution of transmission $m$ to the data obtained from the array. Now, it is generally accepted that the principle of superposition applies to all common propagation media, such as those encountered in radio and sonar [11]. The sensor outputs are therefore obtained by summing $\mathbf{s}_{m}(k)$ over the M transmissions. A noise term is included to model receiver noise.

$$
\underset{\sim}{s}(k)=\sum_{m=1}^{M} s_{m}(k)+\underset{\sim}{w}(k)=\sum_{m=1}^{M} \hat{q}_{m} a_{m}(k)+\underset{\sim}{w}(k)
$$




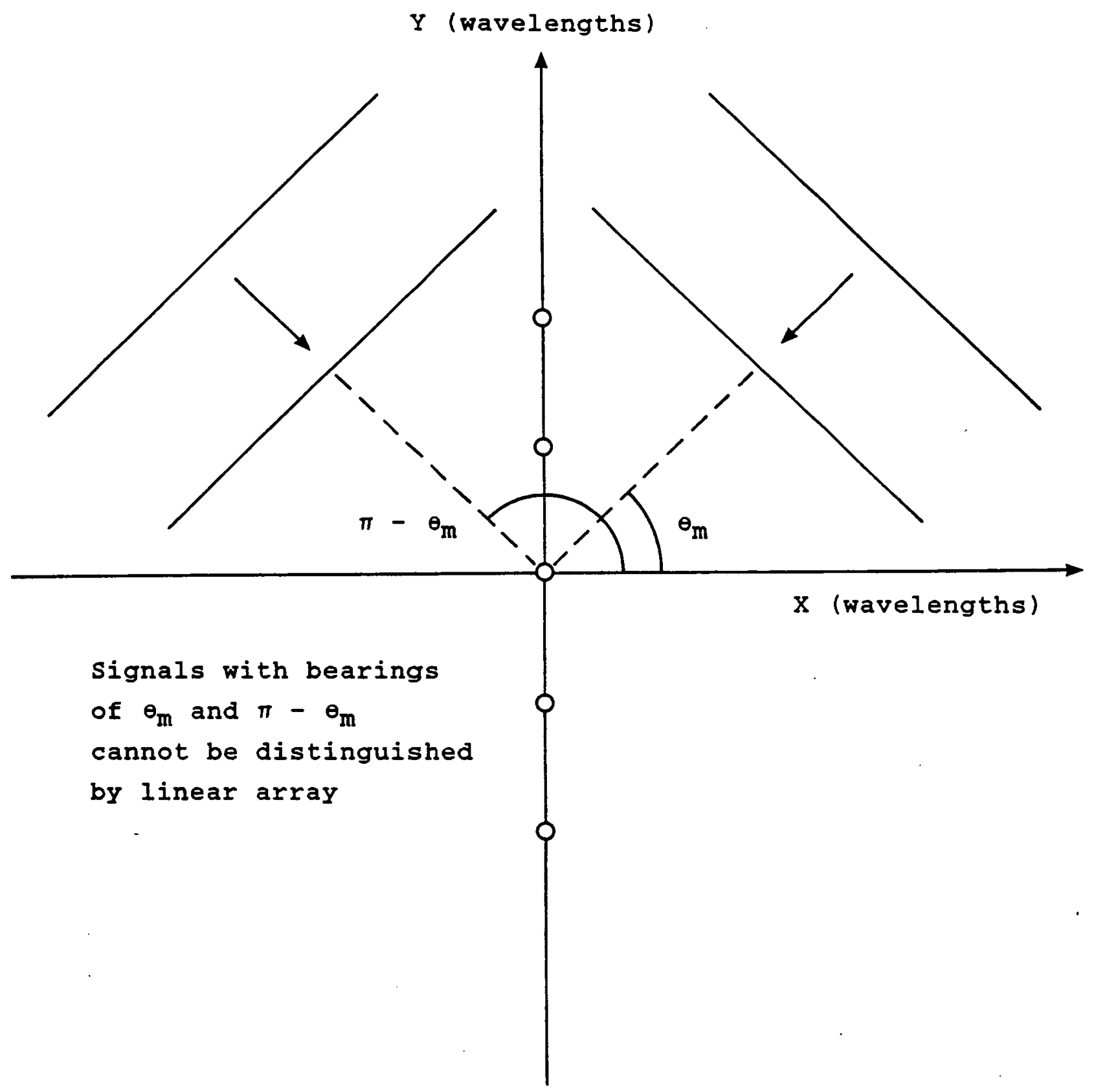

Figure 2.3 Linear array ambiguities 
where

$$
\begin{aligned}
& \underset{\sim}{s}(k)=\left[\begin{array}{llll}
s_{1}(k) & s_{2}(k) & \ldots & s_{N}(k)
\end{array}\right]^{T} \\
& \underset{\sim}{W}(k)=\left[\begin{array}{llll}
w_{1}(k) & w_{2}(k) & \ldots & w_{N}(k)
\end{array}\right]^{T}
\end{aligned}
$$

The complex number $s_{n}(k)$ is the $k^{\text {th }}$ sample from sensor $n$ and the column vector $s(k)$ contains the set of samples obtained simultaneously from the $N$ sensors. $\underset{\sim}{s}(k)$ is commonly referred to as a snapshot.

$w_{n}(k)$ is a sample of a complex Gaussian noise process with a mean of zero and a variance of $\sigma_{w}^{2}$. The noise component from a particular sensor is uncorrelated with the noise from other sensors and the signals being received. The properties of the noise are considered later in more detail during the discussion on covariance matrices.

Equation (2.16) can be expressed in matrix form as follows.

$$
\underset{\sim}{s}(k)=\underset{\sim}{\operatorname{Ha}}(k)+\underset{\sim}{\mathbf{w}}(\mathbf{k})
$$

where

$$
\begin{aligned}
& \underset{\sim}{H}=\left[\begin{array}{llll}
\hat{q}_{1} & \hat{q}_{2} & \ldots & \hat{q}_{M}
\end{array}\right] \\
& \underset{\sim}{a}(k)=\left[\begin{array}{llll}
a_{1}(k) & a_{2}(k) & \ldots & a_{M}(k)
\end{array}\right]^{T}
\end{aligned}
$$


$\stackrel{H}{ }$ is an $N$ by $M$ matrix which has the $M$ signal vectors $\hat{q}_{m}$ as its columns and $\underset{\sim}{a}(k)$ is a column vector containing the $M$ phasors $a_{m}(k)$ which describe the signals observed at the origin. Equation (2.19) is the data model for the snapshots $\underset{\sim}{s}(k)$ collected from the array.

\subsection{Properties of the Signal Vectors}

The signal vectors which form the columns of $\underset{\sim}{H}$ span a subspace within $\mathrm{C}^{\mathbf{N}}$, which denotes the $\mathrm{N}$-dimensional vector space containing all column vectors with $\mathrm{N}$ complex elements. Since $\underset{\sim}{H}$ has $M$ columns, the dimension of the subspace is at most $M$ and is normally equal to $M$. The rank of $\underset{\sim}{H}$, denoted by $r(\underset{\sim}{H})$, is equal to the dimension of this subspace.

$$
r(\underset{\sim}{H}) \leqslant M
$$

The rank is less than $M$ if there is any linear dependence within the set of signal vectors. For example, a linear array produces identical signal vectors for the bearings $\theta_{m}$ and $\pi-\theta_{m}$ as noted earlier. If transmissions were present from both of these directions, they would contribute only one dimension to the subspace instead of two. In general, it is assumed that $\underset{\sim}{\mathrm{H}}$ is full rank and 
the effect of lower ranks is discussed later where appropriate.

\subsection{Covariance Matrices}

The remainder of the chapter is devoted to a study of a number of techniques for performing bearing estimation. Given the snapshots $\underset{\sim}{s}(k)$ and the sensor positions $\hat{\sim}_{n}$, the aim of the process is to deduce the bearings of the signals being received. This may be viewed as an attempt to recover the coefficients of the underlying data model. In particular, if the matrix $\underset{\sim}{H}$ is discovered then the signal vectors become available, which in turn imply the signal bearings.

The first step in the analysis of the snapshots is to form the covariance matrix $\underset{\sim}{R}$, which is defined as follows.

$$
\underset{\sim}{R}=E[\underset{\sim}{s}(k) \underset{\sim}{s} H(k)]
$$

The operator $\mathrm{E}[\mathrm{]}$ denotes the expected value and the superscript $H$ indicates the Hermitian transpose. In practice, it is impossible to obtain the expected value precisely and $\underset{\sim}{R}$ must be approximated by averaging over a finite number of snapshots as follows. 


$$
\underset{\sim}{R} \simeq \frac{1}{\bar{k}} \sum_{k=1}^{R} \underset{\sim}{s}(k) \underset{\sim}{s}(k)
$$

Recalling the definition of the snapshot $\underset{\sim}{s}(k)$ given in (2.19), $\underset{\sim}{R}$ must depend on $\underset{\sim}{H}, \underset{\sim}{a}(k)$, and $\underset{\sim}{\mathbb{w}}(k)$ and the precise relationship can be discovered by substituting the (2.19) into (2.23). Since the signals and the noise are uncorrelated, cross terms involving $\underset{\sim}{a}(k)$ and $\underset{\sim}{w}(k)$ are assumed to vanish.

$$
\begin{aligned}
& \underset{\sim}{\mathrm{R}}=E\left[(\underset{\sim}{\mathrm{Ha}}(\mathbf{k})+\underset{\sim}{\mathbf{w}}(\mathbf{k}))(\underset{\sim}{\mathrm{Ha}}(\mathbf{k})+\underset{\sim}{\mathbf{w}}(\mathbf{k}))^{\mathrm{H}}\right] \\
& =E[\underset{\sim}{H a}(k) \underset{\sim}{a} \mathrm{H}(k) \underset{\sim}{H} \mathrm{H}+\underset{\sim}{w}(k) \underset{\sim}{\mathrm{w}} \mathrm{H}(k)] \\
& =\underset{\sim}{H} E[\underset{\sim}{a}(k) \underset{\sim}{a} \mathrm{H}(k)] \underset{\sim}{H} \mathrm{H}+E\left[\underset{\sim}{w}(k) \underset{\sim}{w^{H}}(k)\right]
\end{aligned}
$$

The two expected values in (2.25) are the covariance matrices of $\underset{\sim}{a}(k)$ and $\underset{\sim}{w}(k)$, which are denoted by $\underset{\sim}{R_{a}}$ and $\underset{\sim}{\mathbb{R}_{W}}$ respectively.

$$
\underset{\sim}{\mathrm{R}}=\operatorname{\sim }_{\sim}^{\mathrm{H}} \underset{\mathrm{a}}{\mathrm{H}^{\mathrm{H}}}+\underset{\sim}{\mathrm{R}}
$$

where

$$
\begin{aligned}
& \underset{\sim}{\mathbf{R}_{a}}=E[\underset{\sim}{a}(k) \underset{\sim}{a} \mathrm{H}(k)] \\
& \underset{\sim}{\mathbf{R}}=E[\underset{\sim}{\mathbf{w}}(k) \underset{\sim}{\mathrm{w}} \mathrm{H}(k)]
\end{aligned}
$$


The matrices $\underset{\sim}{R_{a}}$ and $\underset{\sim}{R_{w}}$ are known as the signal covariance matrix and the noise covariance matrix respectively.

The next three sections make a number of deductions about the matrices $\underset{\sim}{R_{W}}$ and $\underset{\sim}{\mathrm{R}_{a}}$ and the product $\underset{\sim}{\mathrm{R}_{a}} \mathrm{H}_{\sim}^{H}$. These observations are then combined to obtain some important properties of the covariance matrix $\underset{\sim}{R}$.

\subsection{Properties of the Noise Covariance Matrix}

$\stackrel{\sim}{W}_{W}$ is the covariance matrix of the vector $\underset{\sim}{W}(k)$ and, in common with all other covariance matrices, it is Hermitian (i.e. ${\underset{\sim}{W}}_{W}=\underset{\sim}{H}$ ) [84]. Each element $\left(\underset{\sim}{\mathbb{R}_{W}}\right)_{i j}$ expresses the degree of correlation between the additive noise signals produced by sensors $i$ and $j$. An element $\left(\mathbb{R}_{W}\right)_{n n}$ on the leading diagonal is equal to the power of the noise component from sensor $n$, which is $\sigma_{w}^{2}$ for all of the sensors. Since the noise from different sensors is uncorrelated, the off-diagonal elements are all equal to zero and $\mathrm{R}_{\mathrm{w}}$ is therefore a scaled identity matrix.

$$
\underset{\sim}{R_{W}}=\sigma_{W}^{Z} I
$$

Substituting (2.29) into (2.26), the data model can be re-written as follows. 


$$
\underset{\sim}{R}=\underset{\sim}{H} \underset{\sim}{\mathrm{R}_{\sim}}{\underset{\sim}{H}}^{H}+\sigma_{W}^{2} I
$$

Since an identity matrix is full rank [85], the rank of ${ }_{\sim} \mathrm{W}$ is guaranteed to be equal to $\mathrm{N}$ provided that there is noise present (i.e. $\left.\sigma_{\mathrm{w}}^{2}>0\right)$.

$$
r\left(\mathbb{R}_{W}\right)=N
$$

\subsection{Properties of the Signal Covariance Matrix}

As noted earlier, $\underset{\sim}{\mathrm{R}} \mathrm{a}$ is the covariance matrix of the vector $\underset{\sim}{a}(k)$ which contains the $M$ phasors $a_{m}(k)$. Like $\mathbb{\sim}_{w}$, this matrix is Hermitian. The elements on the leading diagonal of $\mathrm{R}_{\mathrm{a}}$ are the powers of the $M$ signals. Recalling the definition of $a_{m}(k)$ given in $(2.3)$, the power of signal $\mathrm{m}$ is $\mathrm{A}_{\mathrm{m}}^{2}$.

$$
\left(\stackrel{\sim}{\mathrm{a}}_{\mathrm{a}}\right)_{\mathrm{mm}}=\mathrm{A}_{\mathrm{m}}^{2}
$$

An off-diagonal element $\left(\underset{\sim}{R_{a}}\right)_{i j}$ expresses the degree of correlation between signals $i$ and $j$. If the $M$ signals are uncorrelated, the off-diagonal elements are all equal to zero and the matrix is diagonal. Non-zero off-diagonal terms indicate that some correlation is present and if $\left|\left(\sim_{a}\right)_{i j}\right|=A_{i} A_{j}$ (with $i \neq j$ ) then signals $i$ 
and $j$ are completely correlated.

This can only happen if there is fixed phase relationship throughout the sampling period, in which case the two signals must have identical frequencies (i.e. $\omega_{i}=\omega_{j}$ ). In this case, the constant phase difference is given by $\arg \left(\left(\underset{\sim}{R_{a}}\right)_{i j}\right)$, which is equal to $\alpha_{i}-\alpha_{j}$.

The rank of $\underset{\sim}{\mathrm{R}} \mathrm{a}$ is equal to the number of independent signals, which is normally M. If there are any completely correlated pairs, the rank is reduced and the matrix is singular.

$$
r\left(\boldsymbol{R}_{\mathbf{a}}\right) \leqslant M
$$

$\underset{\sim a}{\mathrm{R}}$ is assumed to be full rank unless otherwise stated. Completely correlated signals can be caused by multipath [11]-[15] as illustrated in Figure 2.4. The direct signal from the transmitter is accompanied by reflections from the ground or other objects. Refraction in the atmosphere can produce a similar effect.

Multipath creates a situation in which there are two or more sets of wavefronts arriving at the sensor array from different directions. There are time delays between the signals due to the differing path lengths. If the distances change significantly during the sampling period, 


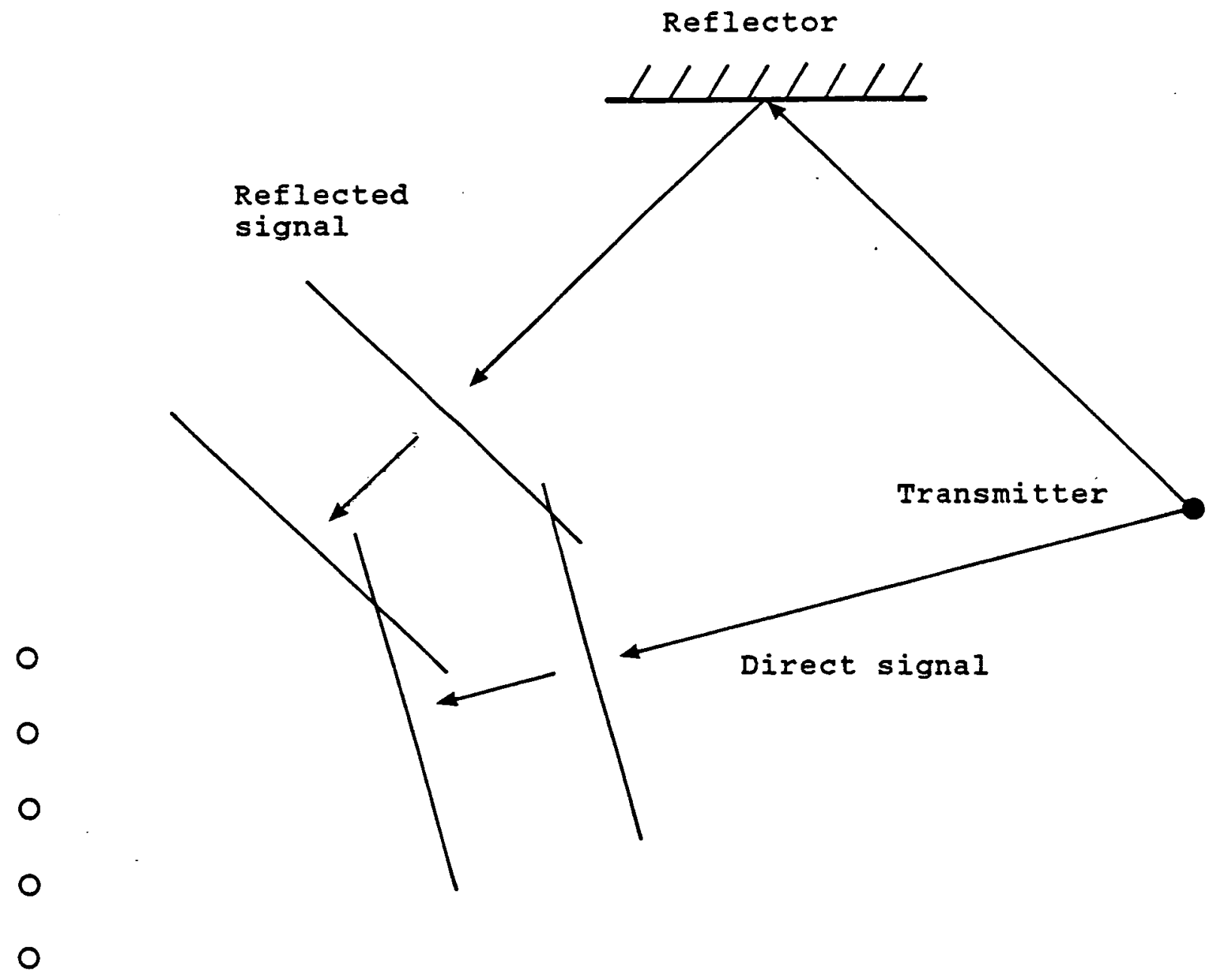

Figure 2.4 Multipath 
the signals drift with respect to each other and are perceived as separate transmissions. If, however, the path lengths remain constant, the signals arriving at the array are completely correlated.

2.10 Properties of the matrix product $\underset{\sim}{\mathrm{H}} \underset{\sim}{\mathrm{a}_{\sim}} \mathrm{H}^{\mathrm{H}}$

From (2.25), $\underset{\sim}{\mathrm{H}} \mathrm{a}_{\sim}^{\mathrm{H}} \mathrm{H}$ is the covariance matrix of the vector formed by the product $\underset{\sim}{\mathrm{Ha}}(\mathrm{k})$ and is therefore Hermitian. Using the rule that the rank of a product of matrices cannot exceed the rank of any factor [86], the rank of $\underset{\sim}{H} \mathrm{\sim}_{a} \underset{\sim}{H}$ must be limited by the rank of $\underset{\sim}{H}$ or $\underset{\sim}{\mathrm{R}}$, whichever is lowest. From (2.22) and (2.33), both $\underset{\sim}{H}$ and $\underset{\sim}{\mathrm{R}}$ have $\mathrm{a}$ rank less than or equal to $M$ and the same limit therefore applies to the rank of $\underset{\sim}{\mathrm{H}} \underset{\sim}{\mathrm{a}} \underset{\sim}{\mathrm{H}}$.

$$
r\left(\underset{\sim}{\mathrm{HR}} \mathrm{a}_{\sim}^{\mathrm{H}} \mathrm{H}\right) \leqslant \mathrm{M}
$$

The rank is normally equal to M unless the rank of $\underset{\sim}{\mathrm{H}}$ is reduced by array geometry anomalies or $\underset{\sim}{\mathrm{R}}$ fails to be full rank due to correlated signals. Since $\underset{\sim}{\mathrm{H}} \underset{\sim}{\mathrm{H}}{ }_{\sim}^{\mathrm{H}}$ is an $\mathrm{N}$ by $\mathrm{N}$ matrix and $M<N$, the matrix cannot be full rank and must therefore be singular. It follows that the determinant of $\underset{\sim}{\mathrm{HR}} \mathrm{a}_{\sim}^{H} \mathrm{H}$ is equal to zero. 


$$
\left|\underset{\sim}{\mathrm{H}} \underset{\sim}{\mathrm{H}} \mathrm{\sim}^{\mathrm{H}}\right|=0
$$

The $\mathrm{N}$ eigenvalues of $\underset{\sim}{\mathrm{H}} \mathrm{a}_{\sim}^{\mathrm{H}} \mathrm{H}^{\mathrm{H}}$ are denoted by $\lambda_{i}, \ldots, \lambda_{\mathrm{N}}$ in ascending order. Since the matrix is Hermitian, all of the eigenvalues are greater than or equal to zero [85]. Furthermore, there must be at least one eigenvalue equal to zero because the matrix is singular and it follows that $\underset{\sim}{\mathrm{HR}} \mathrm{a}_{\sim}^{\mathrm{H}}$ is positive semi-definite. The number of eigenvalues greater than zero is equal to the rank of the matrix, $M$.

$$
\begin{aligned}
& \lambda_{1}^{\prime}=\lambda_{2}^{\prime}=\ldots=\lambda_{\mathrm{N}-\mathrm{M}}^{\dot{ }}=0 \\
& 0<\lambda_{\mathrm{N}-\mathrm{M}+1}^{\dot{*}} \leqslant \lambda_{\mathrm{N}-\mathrm{M}+2} \leqslant \ldots \leqslant \lambda_{\mathrm{N}}
\end{aligned}
$$

\subsection{Properties of the Covariance Matrix}

Like $\underset{\sim}{\mathrm{R}_{w}}, \underset{\sim}{\mathrm{R}_{a}}$, and $\underset{\sim}{\mathrm{H}} \mathbb{a}_{\sim}^{H}{ }^{H}$, the covariance matrix $\underset{\sim}{\mathrm{R}}$ is Hermitian. An element $R_{n n}$ on the leading diagonal is equal to the power of the output from sensor $n$, this being the noise power plus the total power of all of the signals being received.

An off-diagonal element $R_{i j}$ is the cross-correlation between the outputs from sensors $i$ and $j$. These terms contain the phase information that is used to deduce the 
signal bearings. The phase angle $\arg \left(R_{i j}\right)$ is equal to the phase shift between the two sensors, $\hat{\psi}_{i m}-\hat{\psi}_{j m}$, summed over the M signals.

Now, the rank of a sum of matrices cannot exceed the sum of the ranks of the original matrices [86]. From (2.26),

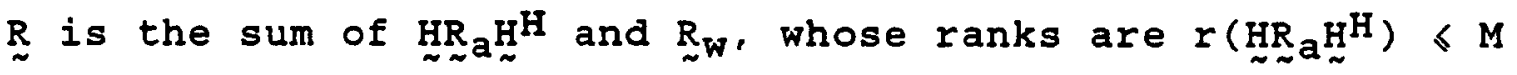
and $r\left({\underset{\sim}{W}}_{W}\right)=N$ according to $(2.34)$ and (2.31). The lowest limit on the rank of $\underset{\sim}{\mathrm{R}}$ occurs when $r\left(\underset{\sim}{\operatorname{H}} \underset{\sim}{\mathrm{R}}{\underset{\sim}{H}}^{H}\right)=1$, in which case $r(\underset{\sim}{R}) \leqslant N+1$. Notice, however, that the maximum possible rank is $N$ since $\underset{\sim}{R}$ is an $N$ by $N$ matrix. Thus the presence of noise usually ensures that $\underset{\sim}{R}$ is full rank.

$$
r(\underset{\sim}{R})=N
$$

The rules governing the eigenvalues of $\underset{\sim}{\mathrm{H}_{\sim}} \mathrm{a}_{\sim}^{\mathrm{H}}$ derived in the last section can be used to achieve some insight into the eigenvalues of $\underset{\sim}{R}$. The first step is to rearrange (2.30) so that $\underset{\sim}{\mathrm{H}} \mathrm{a}_{\sim} \mathrm{H}^{\mathrm{H}}$ is isolated.

$$
\underset{\sim}{\operatorname{Ha}} \mathbf{a}_{\sim}^{H} H=\underset{\sim}{R}-\sigma_{W}^{2} I
$$

From (2.35), the determinant of $\underset{\sim}{\mathrm{H}} \underset{\sim}{\mathrm{R}} \underset{\sim}{\mathrm{H}} \mathrm{H}$ is equal to zero. Substituting (2.39) into (2.35), the determinant of the matrix formed by the expression $\underset{\sim}{\mathrm{R}}-\sigma_{\mathrm{W}}^{2} \mathrm{I}$ must also be equal to zero. 


$$
\left|\underset{\sim}{R}-\sigma_{\mathrm{W}}^{2} \underset{\sim}{I}\right|=0
$$

Equation (2.40) defines the noise power $\sigma_{\mathrm{W}}^{2}$ to be one of the eigenvalues of $\underset{\sim}{R}$, these being $\lambda_{1}, \ldots, \lambda_{N}$ in ascending order. From (2.39), the eigenvalues of $\underset{\sim}{R}$ differ from those of $\underset{\sim}{\mathrm{HR}} \mathrm{a}_{\sim}^{\mathrm{H}}{ }^{\mathrm{H}}$ by $\sigma_{\mathrm{w}}^{2}[22]$.

$$
\lambda_{n}^{\prime}=\lambda_{n}-\sigma_{w}^{2}
$$

Since the eigenvalues of $\underset{\sim}{\mathrm{HR}}{\underset{\sim}{\mathrm{H}}}_{\sim}^{\mathrm{H}}$ are all greater than or equal to zero, (2.41) implies that the eigenvalues of $\underset{\sim}{\mathbb{R}}$ are all greater than or equal to $\sigma_{\mathrm{W}}^{2}$. Furthermore, (2.40) states that at least one of the eigenvalues of $\underset{\sim}{R}$ is actually equal to $\sigma_{\mathrm{W}}^{2}$. Combining these two observations, the smallest eigenvalue of $\underset{\sim}{R}$ must be equal to $\sigma_{\mathrm{W}}^{2}$.

$$
\lambda_{1}=\sigma_{\mathrm{w}}^{2}
$$

A set of rules for the eigenvalues of $\underset{\sim}{R}$ can be obtained by substituting (2.41) and (2.42) into (2.36) and (2.37).

$$
\begin{aligned}
& \lambda_{1}=\lambda_{2}=\ldots=\lambda_{N-M}=\sigma_{\mathrm{W}}^{2} \\
& \sigma_{\mathrm{W}}^{2}<\lambda_{\mathrm{N}-\mathrm{M}+1} \leqslant \lambda_{\mathrm{N}-\mathrm{M}+2} \leqslant \ldots \leqslant \lambda_{\mathrm{N}}
\end{aligned}
$$

From (2.43), the multiplicity of the smallest eigenvalue is $N$ - $M$ and this provides a means of determining $M$ from the covariance matrix $\underset{\sim}{R}$. When $\underset{\sim}{R}$ is computed by averaging 
a finite number of snapshots using (2.24), the $N-M$ smallest eigenvalues form a cluster as illustrated in Figure 2.5 rather than being precisely equal. The diagram shows the eigenvalues of a covariance matrix formed from 500 snapshots using the computer simulation system described later in this chapter.

It is not always straightforward to determine the number of eigenvalues in the cluster, particularly at poor signal to noise ratios or when partially correlated signals are present. No reliable method is known at present although statistical tests are emerging [87]-[91]. The problem has a similar flavour to that of model order determination in autoregressive spectral analysis [17], [18], [20], $[92]-[94]$.

In view of the relationship between the rank of $\underset{\sim}{\mathrm{HR}} \mathrm{a}_{\sim}^{\mathrm{H}} \mathrm{H}$ and the eigenvalues of $\underset{\sim}{R}$, the estimated value of $M$ is, at best, equal to the rank of $\underset{\sim}{\mathrm{HR}} \mathrm{a}_{\sim}^{\mathrm{H}}{ }^{\mathrm{H}}$. AnY of the problems discussed earlier which reduce the rank of $\underset{\sim}{\mathrm{H}} \underset{\sim}{\mathrm{a}_{\sim}}{ }_{\sim}^{\mathrm{H}}$ cause $M$ to be underestimated accordingly. In the following discussion, it is assumed that $M$ is estimated correctly. 


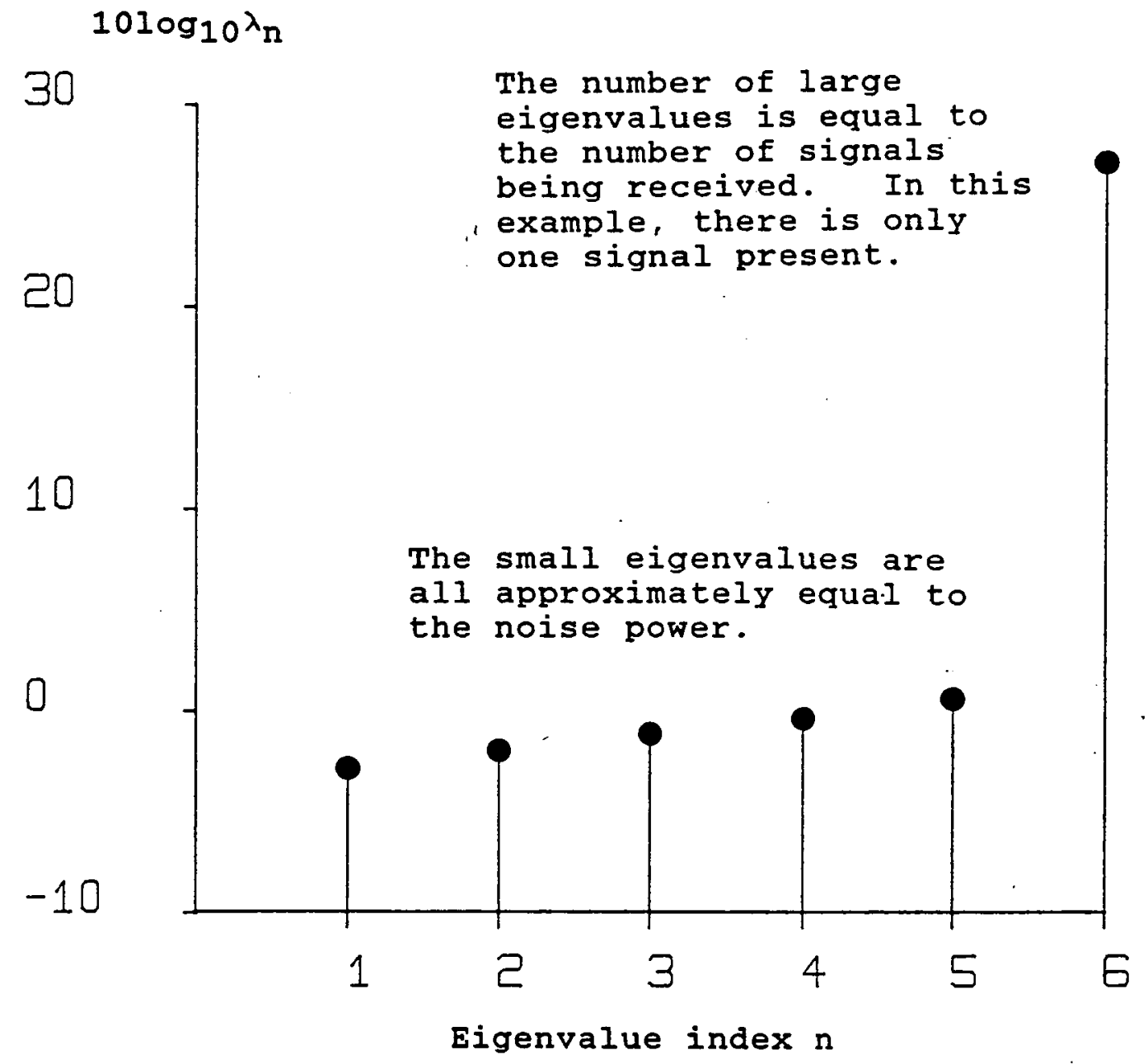

Figure 2.5 Eigenvalues of covariance matrix 


\subsection{Signal and Noise Subspaces}

The $\mathrm{N}$ eigenvectors of $\underset{\sim}{\mathrm{R}}$ are denoted by $\underset{\sim}{v_{1}}, \ldots, v_{N}$, where $\mathbb{v}_{n}$ is the eigenvector corresponding to the eigenvalue $\lambda_{n}$. By definition, the eigenvalues and eigenvectors are related as follows.

$$
\underset{\sim}{\mathrm{R}} \mathrm{n} n=\lambda_{\mathrm{n}} \mathrm{v}_{\mathrm{n}}
$$

The eigenvectors can be related to the data model by substituting $(2.30)$ into $(2.45)$.

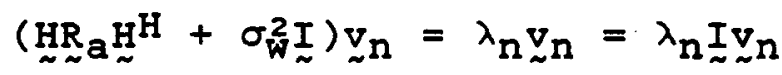

$$
\begin{aligned}
& \Rightarrow \underset{\sim}{\mathrm{H}} \underset{\sim}{\mathrm{a}} \underset{\sim}{\mathrm{H}_{\mathrm{V}}} \mathrm{V}_{\mathrm{n}}=\left(\lambda_{\mathrm{n}}-\sigma_{\mathrm{w}}^{2}\right){\underset{\sim}{\mathrm{n}}}_{\mathrm{N}}
\end{aligned}
$$

From (2.43), $\lambda_{n}-\sigma_{W}^{2}$ is equal to zero for the first $N-M$ eigenvalues since these eigenvalues are all equal to $\sigma_{\mathrm{W}}^{2}$.

$$
\underset{\sim}{\mathrm{HR}} \underset{\sim}{\mathrm{H}^{\mathrm{H}}}{ }_{\sim} \mathrm{n}=\underset{\sim}{0}, \quad 1 \leqslant \mathrm{n} \leqslant \mathrm{N}-\mathrm{M}
$$

Equation (2.47) states that the first $N$ - M eigenvectors of $\underset{\sim}{R}$ are orthogonal to the conjugates of the rows of $\underset{\sim}{\mathrm{HR}} \mathrm{a}_{\sim}^{\mathrm{H}}$. Since $\underset{\sim}{\mathrm{HR}} \mathrm{a}_{\sim}^{\mathrm{H}} \mathrm{H}$ is Hermitian, the conjugate of the $\mathrm{n}^{\text {th }}$ row is equal to the $\mathrm{n}^{\text {th }}$ column and it follows that the first N - M eigenvectors of $\underset{\sim}{R}$ are also orthogonal to the columns of $\underset{\sim}{\mathrm{HR}} \mathrm{a}_{\sim}^{\mathrm{H}}$. 
Since the columns of $\underset{\sim}{\mathrm{H}} \underset{\sim}{\mathrm{H}_{\sim}^{H}} \mathrm{H}$ are linear combinations of the columns of $\underset{\sim}{\mathrm{H}}$, they must lie in the subspace spanned by the columns of $\underset{\sim}{H}$. Assuming that the two matrices have the same rank, the columns of $\underset{\sim}{\mathrm{H}} \mathrm{\sim}_{\sim}^{\mathrm{H}}{ }^{\mathrm{H}}$ must also span that subspace. Since the first $N$ - $M$ eigenvectors of $\underset{\sim}{R}$ are orthogonal to the columns of $\underset{\sim}{\mathrm{H}} \underset{\sim}{\mathrm{R}} \underset{\sim}{\mathrm{H}}$, they must be orthogonal to any vector in the subspace, including the columns of $\underset{\sim}{\mathrm{H}}$.

$$
\underset{\sim}{\mathrm{H}_{\mathrm{V}}} \mathrm{V}_{\mathrm{n}}=\underset{\sim}{0}, \quad 1 \leqslant \mathrm{n} \leqslant \mathrm{N}-\mathrm{M}
$$

As discussed earlier, bearing estimation is essentially an attempt to recover the signal vectors $\hat{q}_{m}$ which form the columns of $\underset{\sim}{H}$. The significance of $(2.48)$ is that it relates the eigenvectors of the covariance matrix obtained from the snapshots $\underset{\sim}{\mathbf{s}}(\mathbf{k})$ to the unknown signal vectors.

Since the $N$ eigenvectors of $\underset{\sim}{R}$ are orthogonal to each other, they must span the whole of $\mathrm{CN}^{N}$. Now, the discussion has shown that the signal vectors $\hat{q}_{m}$ are orthogonal to the subspace spanned by the first $N-M$ eigenvectors. The signal vectors must therefore lie in the M-dimensional subspace spanned by the remaining $M$ eigenvectors $\mathbb{v}_{N-M+1}, \ldots, V_{N}$, which is referred to as the signal subspace. The orthogonal subspace spanned by the first $N-M$ eigenvectors $\nabla_{1}, \ldots, V_{N}-M$ is the noise subspace and has dimension $N-M$. 


\subsection{The MUSIC Algorithm}

Schmidt's MUSIC (Multiple Signal Classification) algorithm [11], [16], [22] is a bearing estimation technique which exploits the concept of signal and noise subspaces. The algorithm generates signal vectors for a range of bearings and tests each one to determine whether or not it lies in the signal subspace. If the signal vector for a particular bearing lies entirely in the signal subspace, it is assumed that a transmission is being received from that direction.

The signal vector for bearing $\theta$ is $\underset{q}{q}(\theta)$, which is calculated in the same way as the unknown signal vectors in the data model, i.e. $\hat{q}\left(\theta_{m}\right)=\hat{q}_{m}$. In Figure $2.6,{\underset{\sim}{s}}_{s}(\theta)$ and $\mathrm{p}_{\mathrm{w}}(\theta)$ are the projections of $\hat{\mathrm{q}}(\theta)$ onto the signal and noise subspaces respectively. The axes have been omitted in order to simplify the diagram.

If $\hat{q}(\theta)$ lies entirely in the signal subspace then the length (squared) of the projection onto the noise subspace, denoted by $\left\|\underline{\sim}_{w}(\theta)\right\|^{2}$, is equal to zero. Meanwhile, the length (squared) of the projection onto the signal subspace, denoted by $\left\|p_{s}(\theta)\right\|^{2}$, reaches a maximum, being equal to $\|\hat{q}(\theta)\|^{2}$. If $\theta$ is swept over a range of directions, the bearings of the signals being received are marked by peaks in $\left\|p_{s}(\theta)\right\|^{2}$ and notches in $\left\|\underline{w}_{W}(\theta)\right\|^{2}$. 


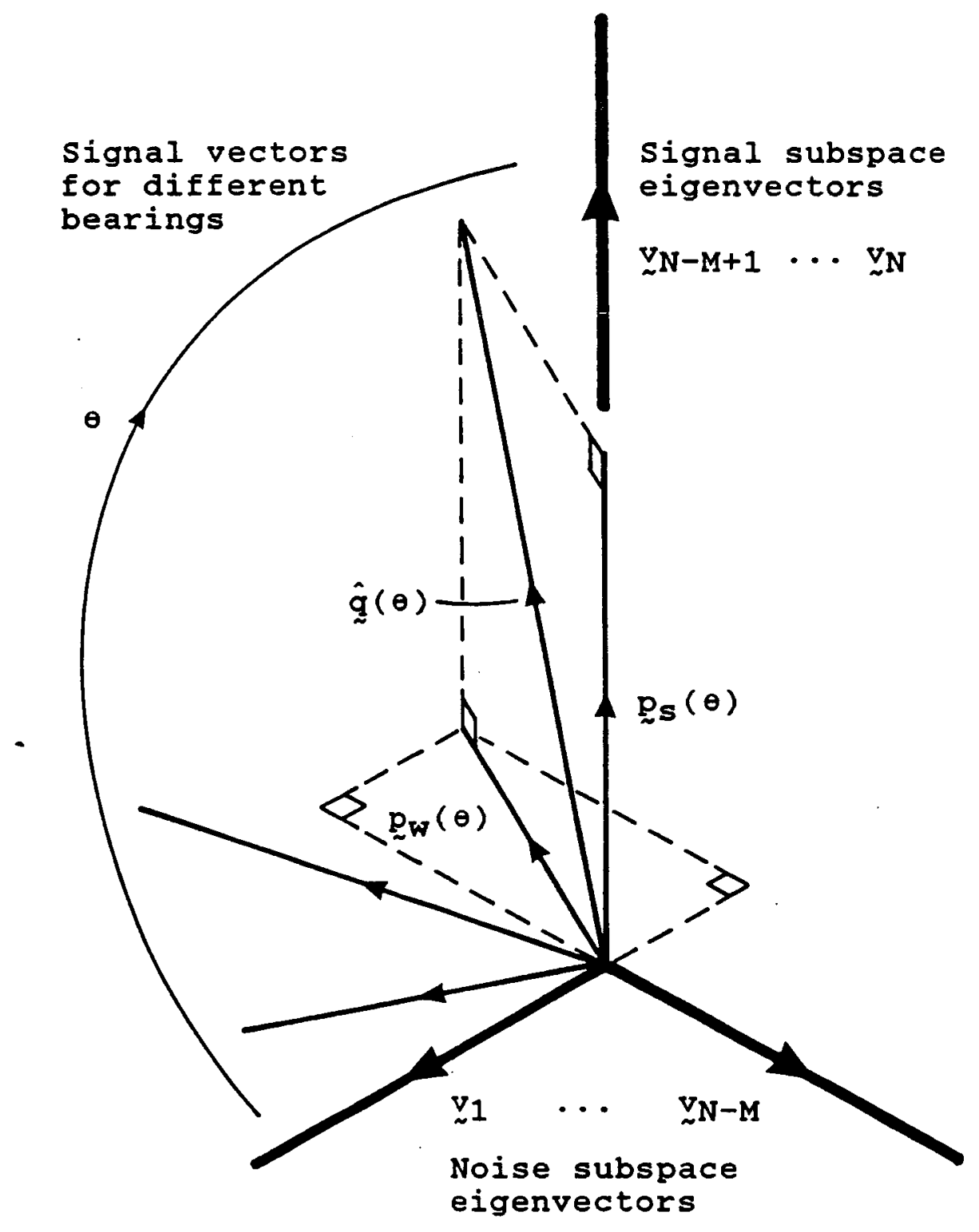

Figure 2.6 Projections onto signal and noise subspaces 
Consider the case where $\hat{q}(\theta)$ lies almost entirely in the signal subspace. If $\theta$ is varied so that $\hat{q}(\theta)$ moves even closer to the subspace, $\left\|p_{w}(\theta)\right\|^{2}$ decreases more rapidly than $\left\|p_{S}(\theta)\right\|^{2}$ increases. This happens because $\left\|p_{W}(\theta)\right\|^{2}$ is determined by the cosine of the angle between $\hat{q}(\theta)$ and the noise subspace whereas $\left\|p_{s}(\theta)\right\|^{2}$ depends on the sine. The gradient of the cosine function reaches its maximum in this area.

It follows that the notches in $\left\|\mathrm{p}_{\mathrm{W}}(\theta)\right\|^{2}$ are sharper than the peaks in $\left\|p_{s}(\theta)\right\|^{2}$, making $\left\|p_{w}(\theta)\right\|^{2}$ preferable when forming a bearing spectrum. Thus a signal vector is actually tested by measuring its orthogonality to the noise subspace rather than the extent to which it lies in the signal subspace. Another reason for this choice is that the noise subspace generally has a higher dimension, provided that the number of signals being received is small compared with the number of sensors.

The projection $\mathrm{p}_{w}(\theta)$, which is the component of $\hat{q}(\theta)$ in the noise subspace, is calculated as follows [85].

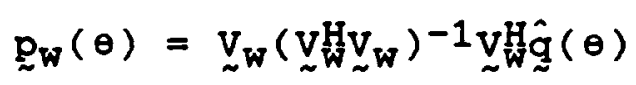

where

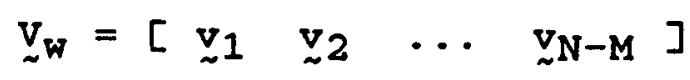


$\underline{\mathrm{V}}_{\mathrm{W}}$ is an $\mathrm{N}$ by $\mathrm{N}$ - M matrix which has the $\mathrm{N}$ - M noise subspace eigenvectors as its columns. Since the eigenvectors are vectors of unit length which are all orthogonal to each other, the columns of $\underline{v}_{\mathrm{W}}$ form an orthonormal set.

$$
\underline{V}_{W} V_{W}=\left(V_{W}^{H} V_{W}\right)^{-1}=I
$$

The expression for the projection ${\underset{\sim}{w}}_{w}(\theta)$ can be simplified by substituting $(2.51)$ into $(2.49)$.

$$
{\underset{\sim}{W}}_{W}(\theta)={\underset{\sim}{W}}_{\sim} \underset{W}{H} \hat{q}(\theta)
$$

The length of the projection, $\left\|p_{w}(\theta)\right\|^{2}$, is the inner product of $p_{w}(\theta)$ and its Hermitian transpose.

$$
\left\|p_{W}(\theta)\right\|^{2}=p_{w}^{H}(\theta) \underset{\sim}{p_{W}}(\theta)
$$

Substituting (2.52) into (2.53) and.simplifying the result using (2.51), the final expression for $\left\|p_{w}(\theta)\right\|^{2}$ is as follows.

$$
\begin{aligned}
& \left\|\underline{\sim}_{W}(\theta)\right\|^{2}=\hat{q}^{H}(\theta) V_{W} V_{\sim} V_{w}^{H} V_{\sim} V_{W}^{H} \hat{q}(\theta) \\
& =\hat{q}^{H}(\theta){\underset{\sim}{W}}_{\sim} \underset{W}{H} \hat{q}(\theta)
\end{aligned}
$$

As explained earlier, $\left\|p_{w}(\theta)\right\|^{2}$ reaches a minimum whenever $\theta$ is equal to the bearing of one of the signals being 
received. It is conventional, however, for the bearings of the signals to be indicated by peaks in the spectrum rather than notches and this is achieved by defining the MUSIC spectrum to be the reciprocal of $\left\|p_{W}(\theta)\right\|^{2}$.

$$
\mathrm{P}_{\mathrm{mu}}(\theta)=\frac{1}{\hat{\mathfrak{q}}^{\mathrm{H}}(\theta) \underset{\sim}{V_{W} \underset{W}{\mathrm{H}} \hat{q}(\theta)}}
$$

The bearing spectrum is generated by evaluating $P_{m u}(\theta)$ over a range of closely spaced values of $\theta . P_{m u}(\theta)$ is always real and is theoretically equal to infinity when $\theta$ equals one of the signal bearings $\theta_{\mathrm{m}}$.

To summarize, the first step in the MUSIC algorithm is to gather the snapshots $\underset{\sim}{\mathbf{s}(k)}$ and form the covariance matrix $\underset{\sim}{R}$. The eigenvalues $\lambda_{n}$ and eigenvectors ${\underset{\sim}{n}}_{n}$ of $\underset{\sim}{R}$ are calculated and the number of signals, $M$, is determined by inspecting the eigenvalues. The matrix $\underset{\sim}{\mathrm{W}}$ is then formed using the first $N$ - M eigenvectors and the bearing spectrum is plotted by evaluating $P_{m u}(\theta)$ over a range of bearings. For each bearing, the signal vector $\hat{q}(\theta)$ is calculated from the sensor positions $\hat{\sim}_{n}$ and the angle $\theta$. Peaks in $P_{m u}(\theta)$ are assumed to indicate the bearings of the signals being received.

The discussion has demonstrated the natural progression from the data model to the idea of signal and noise 
subspaces and from there to MUSIC. The next two sections describe two other techniques for bearing estimation known as conventional beamforming and the minimum energy method. _. Both of these operate on the covariance matrix $\underset{\sim}{\mathbb{R}}$ although the processing is somewhat different from that employed in MUSIC. Following this, the computer simulation system is introduced and bearing spectra for all three techniques are presented.

\subsection{Conventional Beamforming}

This section describes the conventional beamforming technique for bearing estimation [16], which is essentially a spatial Fourier transform similar in form to the discrete Fourier transform used in time series analysis [17], [18]. The discrete Fourier transform of the output from sensor $\mathrm{n}$ is defined as follows.

$$
\tilde{s}(\omega)=\sum_{k=1}^{R} s_{n}(k) \exp \left\{-j 2 \pi(k-1) \frac{\omega}{\omega_{s}}\right\}
$$

In the spatial Fourier transform, the sequence of samples $s_{n}(1), \ldots, s_{n}(R)$ obtained from one sensor at different times is replaced by the set of samples $s_{1}(k), \ldots, s_{N}(k)$ 
collected from the $N$ sensors at the same time.

The term $2 \pi(k-1) \omega / \omega_{s}$ in $(2.56)$ is the phase angle of a sinusoid with frequency $\omega$ observed at the time of the $k^{\text {th }}$ sample and can be regarded as a phase shift due to the time which has elapsed since the first sample was taken. In the spatial Fourier transform, the term is replaced by $\hat{\Psi}_{n}(\theta)$, which is the phase shift from the position of the reference sensor (sensor 1) to that of sensor $n$. This is defined in the same way as the phase shifts used in the data model, i.e. $\hat{\psi}_{n}\left(\theta_{m}\right)=\hat{\psi}_{n m}$. Combining these observations, the definition of the spatial Fourier transform is as follows.

$$
s(\theta)=\sum_{n=1}^{N} s_{n}(k) \exp \left\langle-j \hat{\psi}_{n}(\theta)\right\rangle
$$

Noting that $\hat{q}_{n}^{*}(\theta)=\exp \left(-j \hat{\psi}_{n}(\theta)\right)$ where * denotes complex conjugation, (2.57) can be expressed in matrix form.

$$
S(\theta)=\hat{q}^{H}(\theta) \underline{\sim}(k)
$$

The conventional beamforming bearing spectrum is obtained by taking the expected value of $|S(\theta)|^{2}$. 


$$
\begin{aligned}
P_{b f}(\theta) & =E\left[|S(\theta)|^{2}\right] \\
& =E\left[\hat{\sim}^{H}(\theta) \underset{\sim}{s}(k){\underset{\sim}{s}}^{H}(k) \underset{\sim}{q}(\theta)\right] \\
& =\hat{\sim}^{H}(\theta) E\left[\underset{\sim}{s}(k){\underset{\sim}{s}}^{H}(k)\right] \underset{\sim}{q}(\theta)
\end{aligned}
$$

Notice that (2.59) contains the expected value $E\left[s(k) \underline{\sim}^{H}(k)\right]$, which is the definition of the covariance matrix $\underset{\sim}{R}$ given earlier in (2.23). This observation allows (2.59) to be re-written as follows.

$$
\mathrm{P}_{\mathrm{bf}}(\theta)=\hat{q}^{\mathrm{H}}(\theta) \underset{\sim}{\operatorname{R}} \hat{q}(\theta)
$$

The bearing spectrum is generated by evaluating $P_{b f}(\theta)$ over a range of values of $\theta$. It can be shown that the resulting spectrum has units of power [16] and this is confirmed by the analysis presented in Chapter 3.

Conventional beamforming is computationally less expensive than MUSIC since the eigenvalues and eigenvectors of the covariance matrix $\underset{\sim}{R}$ do not have to be computed. This reduction in cost is, however, accompanied by a reduction in the quality of the bearing spectrum [16], [25] and the simulation results at the end of the chapter confirm the general superiority of the MUSIC technique. 


\subsection{The Minimum Energy Method}

Like the discrete Fourier transform, conventional beamforming produces spectra with large sidelobes [16], meaning that $P_{b f}(\theta)$ can be significantly influenced by signals with bearings other than $\theta$. The choice of $\hat{q}(\theta)$ as the steering vector in (2.60) sets the gain in the direction $\theta$ without making any attempt to suppress signals being received from other directions.

This section describes the minimum energy method [16], which attempts to minimize the received power subject to the constraint that the gain in the direction $\theta$ is equal to 1 . The result is that the influence of signals from other directions is reduced and the quality of the bearing spectrum is improved. In the minimum energy method, $\hat{q}(\theta)$ is replaced with a new steering vector $\underset{\sim}{\hat{b}}(\theta)$. By analogy with the expression for $P_{b f}(\theta)$ given in $(2.60)$, the received power is as follows.

$$
P_{\text {me }}(\theta)=\hat{\sim}^{H}(\theta) \underset{\sim}{\operatorname{Rb}} \hat{\sim}(\theta)
$$

The vector $\underset{\sim}{\hat{b}}(\theta)$, which is chosen so as to minimize $P_{m e}(\theta)$, can be regarded as a summation of signal vectors for different bearings using complex weights. Recalling the definition of the spatial Fourier transform given in (2.58), the product $\underset{\sim}{\hat{q}}(\theta) \underset{\sim}{\hat{b}}(\theta)$ expresses the magnitude and 
phase of the component of $\underset{\sim}{\hat{b}}(\theta)$ at the bearing $\theta$. If the weighting of this component is forced to be 1 , the system must have unit gain in the direction $\theta$ and the constraint is therefore expressed as follows.

$$
\hat{\sim}^{H}(\theta) \underset{\sim}{\hat{b}}(\theta)=1
$$

The minimization of $P_{m e}(\theta)$ subject to this constraint is performed using a Lagrange multiplier [16]. The aim is to minimize the following expression, which incorporates both the definition of $P_{m e}(\theta)$ given in $(2.61)$ and the constraint sepcified in (2.62).

$$
F={\underset{\sim}{b}}^{H}(\theta) \underset{\sim}{R} \hat{\sim}(\theta)+\beta\left(\underset{\sim}{\hat{q}}{ }^{H}(\theta) \underset{\sim}{\hat{b}}(\theta)-1\right)
$$

$\beta$ is the Lagrange multiplier. The value of $\hat{\mathrm{b}}(\theta)$ which minimizes $F$ is found by differentiating $F$ with respect to $\hat{\mathrm{b}}(\theta)$ and setting the derivative to zero. Since the expression contains a reference to $\hat{\mathrm{b}}^{H}(\theta)$, the derivative of $F$ with respect to the conjugate of $\underset{\sim}{\hat{b}}(\theta)$ must also be considered. The calculus can, however, be simplified by treating $\underset{\sim}{\hat{b}}(\theta)$ and its conjugate $\hat{\sim}^{*}(\theta)$ as independent variables [16]. The differentiation of $F$ with respect to $\hat{\mathrm{b}}(\theta)$ splits naturally into the following two parts.

$$
\frac{\partial}{\partial \underset{\sim}{\hat{b}}(\theta)} F=\frac{\partial}{\partial \hat{b}(\theta)}\langle\underset{\sim}{\hat{b}} H(\theta) \underset{\sim}{\operatorname{R} \hat{b}}(\theta)\rangle+\frac{\partial}{\partial \underset{\sim}{\hat{b}}(\theta)}\left\langle\beta\left({\underset{\sim}{q}}^{H}(\theta) \underset{(2.64)}{\hat{b}}(\theta)-1\right)\right\rangle
$$

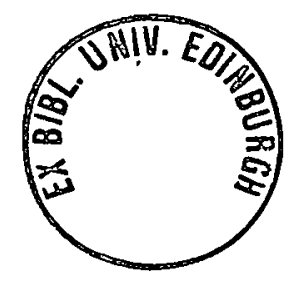


A similar expression is obtained for the derivative with respect to $\hat{\mathrm{b}}^{*}(\theta)$. The, first differentiation in the right hand side of (2.64) involves the quadratic form $\hat{\sim}^{H}(\theta) \underset{\sim}{\operatorname{Rb}} \hat{\sim}(\theta)$, which can be expanded as follows.

$$
\hat{\sim}^{H}(\theta) \underset{\sim}{\operatorname{Rb}} \hat{\sim}(\theta)=\sum_{i=1}^{N} \sum_{j=1}^{N} \hat{b}_{i}^{*}(\theta) R_{i j} \hat{b}_{j}(\theta)
$$

The next step is to find the partial derivatives of $\hat{\sim}^{\hat{b}}(\theta) \underset{\sim}{\operatorname{Rb}} \hat{\sim}(\theta)$ with respect to the elements $\hat{b}_{n}(\theta)$ and $\hat{b}_{n}^{*}(\theta)$. According to the expansion given in (2.65), this involves differentiating $\hat{b}_{n}(\theta)$ with respect to $\hat{b}_{n}^{*}(\theta)$ and vice versa. These derivatives are normally considered to be undefined, there being no analytic function which relates a complex number to its conjugate [95].

However, since $\hat{b}_{n}(\theta)$ and $\hat{b}_{n}^{*}(\theta)$ are being treated as independent variables, the derivative of one with respect to the other can be assumed to be zero. A complex gradient operator whose properties support this assumption has been reported in the adaptive array literature [95]. The partial derivatives of $\underset{\sim}{\hat{b}} H(\theta) \underset{\sim}{\operatorname{Rb}} \hat{\sim}(\theta)$ with respect to $\hat{b}_{n}(\theta)$ and $\hat{b}_{n}^{*}(\theta)$ are therefore as follows.

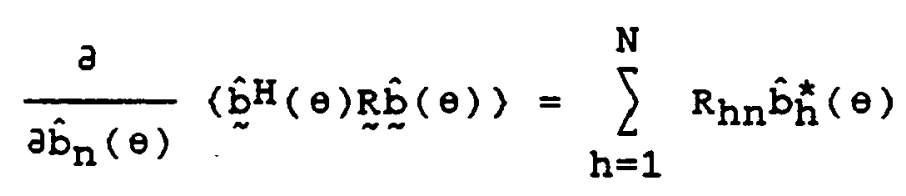




$$
\left.\frac{\partial}{\partial \hat{b}_{n}^{*}(\theta)} \mid \hat{\sim}^{\hat{b}} H(\theta) \underset{\sim}{\operatorname{Rb}} \hat{\sim}(\theta)\right)=\sum_{h=1}^{N} R_{n h} \hat{b}_{h}(\theta)
$$

Since $\underset{\sim}{R}$ is Hermitian, $R_{n h}=R_{h n}^{*}$ and it follows that the term $R_{n h} \hat{b}_{h}(\theta)$ on the right hand side of $(2.67)$ is equal to $\left\langle R_{h n} \hat{b}_{h}^{*}(\theta)\right)^{*}$, which is the complex conjugate of the corresponding term in (2.66). The derivatives of $\hat{b}^{H}(\theta) \underset{\sim}{R} \hat{b}(\theta)$ with respect to $\hat{b}_{n}(\theta)$ and $\hat{b}_{n}^{*}(\theta)$ are therefore complex conjugates and if one of them is set to zero then the other is guaranteed to be zero as well. It is thus sufficient to consider only the derivative with respect to $\hat{b}_{n}(\theta)$.

The derivative of $\hat{\sim}^{\hat{b}} H(\theta) \underset{\sim}{\operatorname{R}} \hat{\sim}(\theta)$ with respect to $\underset{\sim}{\vec{b}}(\theta)$ is a vector containing the derivatives with respect to the individual elements $\hat{b}_{n}(\theta)$. Noting that the summation in (2.66) selects the elements $R_{1 n}, R_{2 n}, \ldots, R_{N n}$, which form the $n^{t h}$ column of $\underset{\sim}{R}$, the derivative with respect to $\hat{b}(\theta)$ can be expressed as follows in matrix form.

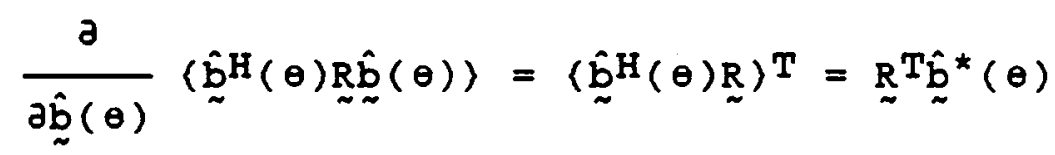

There is a popular misconception that the right hand side of $(2.68)$ should be $2 \underset{\sim}{\mathrm{T}} \hat{\mathrm{b}}^{*}(\theta)$ by analogy with the case where $\underset{\sim}{\hat{b}}(\theta)$ is real and $\underset{\sim}{R}$ is symmetric [95]. Although the 
derivation presented here is more correct, both versions produce the same final answer since the superfluous factor of two is accommodated when solving for $\beta$. [95]

The differentiation of $\beta\left({\underset{\sim}{q}}^{H}(\theta) \underset{\sim}{\hat{b}}(\theta)-1\right)$ in $(2.64)$ is more straightforward and the derivative with respect to $\hat{b}^{*}(\theta)$ is zero since the expression contains $\underset{\sim}{\hat{b}}(\theta)$ but not $\hat{\sim}^{H}(\theta)$. The derivative with respect to $\underset{\sim}{\hat{b}}(\theta)$ is as follows.

$$
\frac{\partial}{\partial \hat{\sim} \hat{b}(\theta)}\left\langle\beta\left({\underset{\sim}{\hat{q}}}^{H}(\theta) \underset{\sim}{\hat{b}}(\theta)-1\right)\right\rangle=\beta \hat{\sim}^{*}(\theta)
$$

The derivative of $F$ with respect to $\underset{\sim}{\hat{b}}(\theta)$ is found by substituting $(2.68)$ and $(2.69)$ into $(2.64)$ and the value of $\underset{\sim}{\hat{b}}(\theta)$ which minimizes $F$ and $P_{m e}(\theta)$ is sought by setting the derivative to zero.

$$
\underset{\sim}{\mathrm{R}} \mathrm{T}^{\star} \hat{\sim}^{\star}(\theta)+\beta \hat{\tilde{q}}^{\star}(\theta)=\underset{\sim}{0}
$$

Since $\underset{\sim}{R}$ is Hermitian, $\underset{\sim}{\mathbb{R} T}=\underset{\sim}{R^{*}}$ and $(2.70)$ can be solved for $\underset{\sim}{b}(\theta)$ by conjugating both sides, pre-multiplying by $\mathrm{R}^{-1}$, and rearranging.

$$
\underset{\sim}{\hat{b}}(\theta)=-\beta \underset{\sim}{R}-1 \underset{q}{q}(\theta)
$$


The Lagrange multiplier $\beta$ can now be found by substituting (2.71) into the constraint equation (2.62) and rearranging.

$$
\beta=-\frac{1}{{\underset{\sim}{q}}^{H}(\theta) \underset{\sim}{R}-1 \underset{\sim}{q}(\theta)}
$$

The following expressions for $\underset{\sim}{\hat{b}}(\theta)$ and ${\underset{\sim}{b}}^{H}(\theta)$ are obtained by substituting $(2.72)$ into $(2.71)$.

$$
\begin{aligned}
& \underset{\sim}{\hat{b}}(\theta)=\frac{\stackrel{\sim}{R}^{-1} \hat{q} \underset{\sim}{\hat{q}}(\theta)}{{\underset{\sim}{q}}^{H}(\theta) \underset{\sim}{R^{-1}} \hat{q}(\theta)}
\end{aligned}
$$

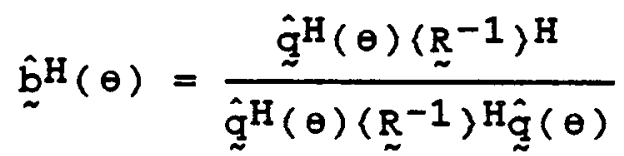

The final step in the derivation is to substitute the expressions for $\underset{\sim}{\hat{b}}(\theta)$ and $\underset{\sim}{\hat{b}} \mathrm{H}(\theta)$ obtained from $(2.73)$ and (2.74) into the definition of $P_{m e}(\theta)$ given in (2.61).

$$
P_{m e}(\theta)=\frac{1}{\hat{\underline{q}}^{H}(\theta) \underline{\sim}^{-1} \hat{q}(\theta)}
$$

The bearing spectrum is produced by evaluating $P_{m e}(\theta)$ over a range of values of $\theta$. Like conventional beamforming, the minimum energy method generates a spectrum which has units of power [16] and this is confirmed by the theoretical expressions derived in chapter 3. 
Since the inverse of the covariance matrix $R$ has to be computed, the minimum energy method is more computationally expensive than conventional beamforming although it is not as expensive as MUSIC. Not surprisingly, the minimum energy method tends to perform better than conventional beamforming but not as well as MUSIC [16], [25]. The remainder of this chapter compares the three techniques using computer simulations.

\subsection{Computer Simulation of Bearing Estimation Techniques}

A suite of computer programs was developed to allow the bearing estimation techniques described in this chapter to be evaluated over a range of operating conditions and to provide a means of checking the original work presented in Chapters 3 and 4 . This section provides a brief description of the simulation system and includes a number of bearing spectra generated using the conventional beamforming, minimum energy, and MUSIC techniques. Features of the system which are specific to the work described in chapters 3 and 4 are discussed in those chapters at the appropriate points.

The programs in the simulation suite may be broady divided into those which model the sampling process and 
those which apply the bearing estimation techniques. The simulation of the sampling process takes the sensor positions and signal parameters as its inputs and produces the covariance matrix $\underset{\sim}{\mathrm{R}}$ as its output.

The $X$ and $Y$ coordinates of each sensor are specified in wavelengths and the array is always positioned so that the reference sensor (sensor 1 ) is at the origin. The sensor positions for linear and circular arrays are generated automatically from parameters such as the number of sensors in the array and the spacing between adjacent sensors. Arbitrary array geometries may be defined by specifying the sensor positions manually.

Each of the received signals is described by specifying the bearing $\theta_{m}$ in degrees, the power $A_{m}^{2}$ in decibels, the initial phase angle $\alpha_{m}$ in degrees, and the relative frequency $\omega_{\mathrm{m}} / \omega_{\mathrm{c}}$. As noted earlier, the frequencies must all be slightly different in order to ensure that the signals drift with respect to each other. Finally, the noise power $\sigma_{\mathrm{W}}^{2}$ is specified in decibels.

The covariance matrix $\underset{\sim}{R}$ is calculated by applying the data model developed earlier in this chapter. The unit vectors $z_{m}$ are obtained directly from the bearings $\theta_{m}$. The signal vectors $\hat{q}_{m}$ are then derived by combining the vectors $z_{m}$ with the sensor positions $\hat{\sim}_{n}$ using (2.5), 
$(2.6),(2.8)$, and $(2.15)$. The matrix $\underset{\sim}{\mathrm{H}}$ is formed by placing the signal vectors $\hat{q}_{m}$ side by side as specified in $(2.20)$.

The simulation system provides two different techniques for calculating the covariance matrix $\underset{\sim}{R}$, the first of which involves averaging over a finite number of snapshots. In this case, the samping rate $\omega_{s} / \omega_{c}$ and the number of snapshots $K$ must be specified. From (2.19), the $k^{\text {th }}$ snapshot $\underset{\sim}{s}(k)$ is given by $\underset{\sim}{s}(k)=\underset{\sim}{\operatorname{Ha}}(k)+\underset{\sim}{\mathbb{W}}(k)$ where $\underset{\sim}{a}(k)$ is calculated using (2.3) and (2.21) from the signal frequecies $\omega_{m} / \omega_{C}$, the amplitudes $A_{m}$, the phase angles $\alpha_{m}$, the sampling rate $\omega_{s} / \omega_{c}$, and the sample number k.

The elements $w_{n}(k)$ of the noise vector $\underset{\sim}{\mathbb{W}}(k)$ are created using the drand48 random number generator [96] which is supplied with the Unix operating system. This function produces random. numbers with a flat distribution. Now, $w_{n}(k)$ is a complex number whose real and imaginary parts both have a normal distribution with a mean of zero and a variance of $\sigma_{\mathrm{w}}^{2}$. It follows that the phase angle $\arg \left\langle w_{n}(k)\right\rangle$ has a flat distribution over the range 0 to $2 \pi$ while the magnitude $\left|w_{n}(k)\right|$ has a Rayleigh distribution $[97]$. 
The procedure for producing $\mathrm{w}_{n}(\mathrm{k})$ using a random number generator with a flat distribution is therefore as follows. For each noise term $w_{n}(k)$, the drand48 function is called twice and the resulting values are scaled so as to produce two real numbers $P_{1}$ and $P_{2}$ such that $0 \leqslant P_{1}<1$ and $0 \leqslant e_{2}<1$. Thus $2 \pi \rho_{1}$ has a flat distribution over the range 0 to $2 \pi$ while $\checkmark\left(-\ln \left(1-\rho_{2}\right)\right\}$ has a Rayleigh distribution [98]. The noise term $w_{n}(k)$ is therefore given by $w_{n}(k)=\left\langle\cos 2 \pi e_{1}+j \sin 2 \pi e_{1}\right) \sigma_{w} v\left(-\ln \left(1-e_{2}\right)\right\rangle$.

Once the sequence of snapshots $\underset{\sim}{s}(k)$ has been obtained, the covariance matrix $\underset{\sim}{\mathrm{R}}$ is calculated by averaging the product $\mathbf{s}(k) \underset{\sim}{\mathrm{H}}(k)$ over the $\mathrm{K}$ snapshots using (2.24).

The simulation system also provides an alternative technique for calculating the covariance matrix in which the expected value $E\left[\underset{\sim}{s}(k){\underset{\sim}{s}}^{H}(k)\right]$ is obtained directly from the signal parameters and sensor positions. In practical terms, this corresponds to averaging over an infinite number of snapshots and thus provides a way of eliminating the errors caused by using a finite number.

In this case, the covariance matrix is calculated using (2.30), which states that $\underset{\sim}{R}=\underset{\sim}{\mathrm{H}} \underset{\sim}{\mathrm{R}} \underset{\sim}{\mathrm{H}}+\sigma_{\mathrm{W}}^{2} I . \quad$ From (2.32), each element $\left(\underset{\sim}{\mathbb{R}_{a}}\right)_{\mathrm{mm}}$ on the leading diagonal of $\underset{\sim}{\mathbb{R}_{a}}$ is set to the power $A_{m}^{2}$ of the corresponding signal. Normally, all of the signals have different frequencies and the 
off-diagonal element $\left(\underset{\sim}{R_{a}}\right)_{h i}$ are all set to zero. If, however, signals $h$ and $i$ are specified as having the same frequency then $\left(\underset{\sim}{R_{a}}\right)_{h i}$ is set to $A_{h} A_{i} \exp \left(j\left(\alpha_{h}-\alpha_{i}\right)\right)$.

Bearing spectra are produced by evaluating the expressions for $P_{b f}(\theta), P_{m e}(\theta)$, and $P_{m u}(\theta)$ given in $(2.60),(2.75)$, and (2.55). All of the spectra in this thesis cover the full range of bearings from 0 to 360 degrees with a spacing of 0.5 degrees between adjacent points.

The matrix inversion required by the minimum energy method is provided using a standard Gaussian elimination procedure [85] while the eigenvalue decomposition for MUSIC is performed by an LZ algorithm [99]. The MUSIC algorithm also requires an estimate of the number of signals being received and this is obtained by inspecting the eigenvalues as discussed earlier. The ratio $\lambda_{n+1} / \lambda_{n}$ is calculated for each value of $n$ in the range 1 to $N-1$ and the value of $n$ which maximises this ratio is assumed to be equal to $N-M$, thus allowing $M$ to be determined.

As noted earlier, however, the estimation of $M$ from the eigenvalues is somewhat unreliable, even when more sophisticated tests are used. The simulation system therefore allows the user to override the estimated value by stating the number of signals explicitly. All of the MUSIC spectra in this thesis were produced using this 
facility, although the estimated value was actually correct in most cases.

For the sake of consistency, all of the simulation results in this chapter and Chapters 3 and 4 are based on the same array of sensors. This array, which is shown in Figure 2.7, consists of five sensors evenly spaced around the circumference of a circle of radius 0.5 wavelengths with a sixth sensor at the centre of the circle.

Figures $2.8(a), 2.9(a)$, and $2.10(a)$ show bearing spectra generated using the conventional beamforming, minimum energy, and MUSIC techniques when the array was receiving a signal with a bearing of 18 degrees. The power $A_{1}^{2}$ of the signal was $20 \mathrm{~dB}$, its phase angle $\alpha_{1}$ was zero degrees, and its relative frequency $\omega_{1} / \omega_{c}$ was 1 . The noise power $\sigma_{\mathrm{W}}^{2}$ was $0 \mathrm{~dB}$ (giving a signal to noise ratio of $20 \mathrm{~dB}$ ) and the covariance matrix was, formed using 500 snapshots obtained at a sampling rate $\omega_{s} / \omega_{c}$ of 3.4567 snapshots per cycle.

The quantity plotted vertically in each spectrum is $\operatorname{lol}_{10} P(\theta)$ where $P(\theta)$ is $P_{b f}(\theta), P_{m e}(\theta)$ or $P_{m u}(\theta)$ as appropriate. In the case of conventional beamforming and minimum energy, this results in a vertical axis which is calibrated in power in decibels. It should be clear from the discussion earlier that MUSIC does not produce a power 


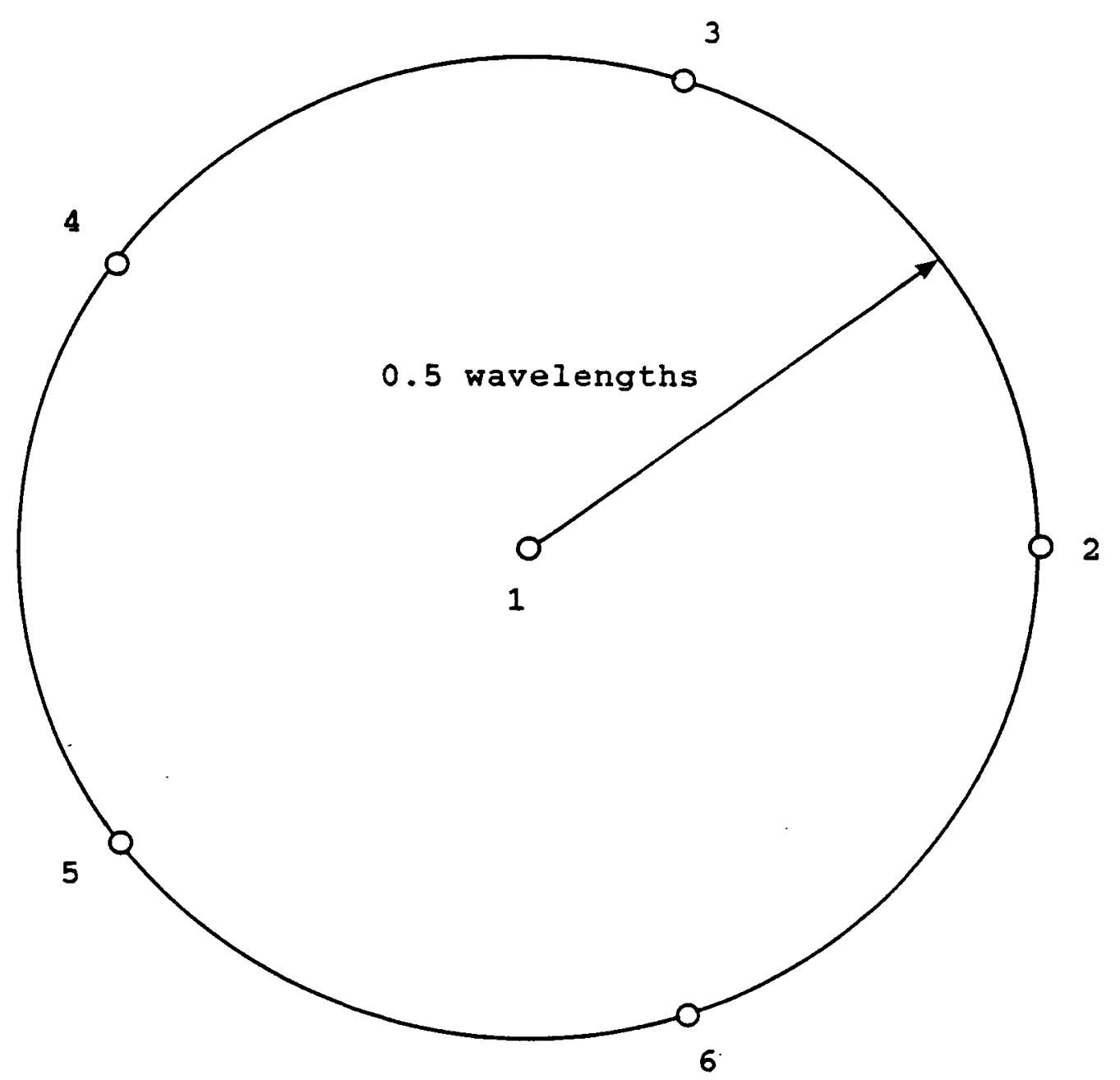

Figure 2.7 Array for computer simulations 
(a) Power (dB)

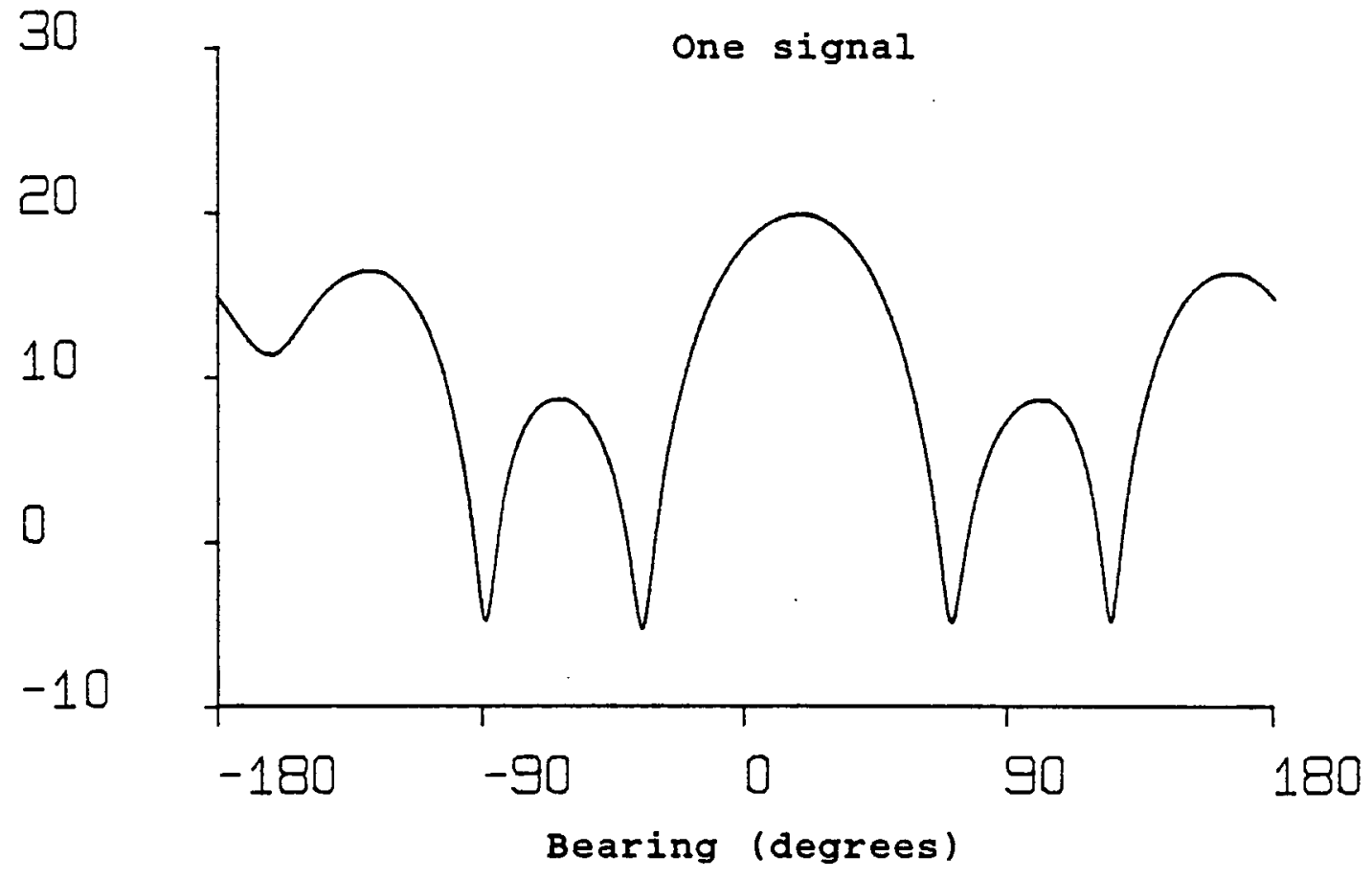

(b) Power (dB)

30

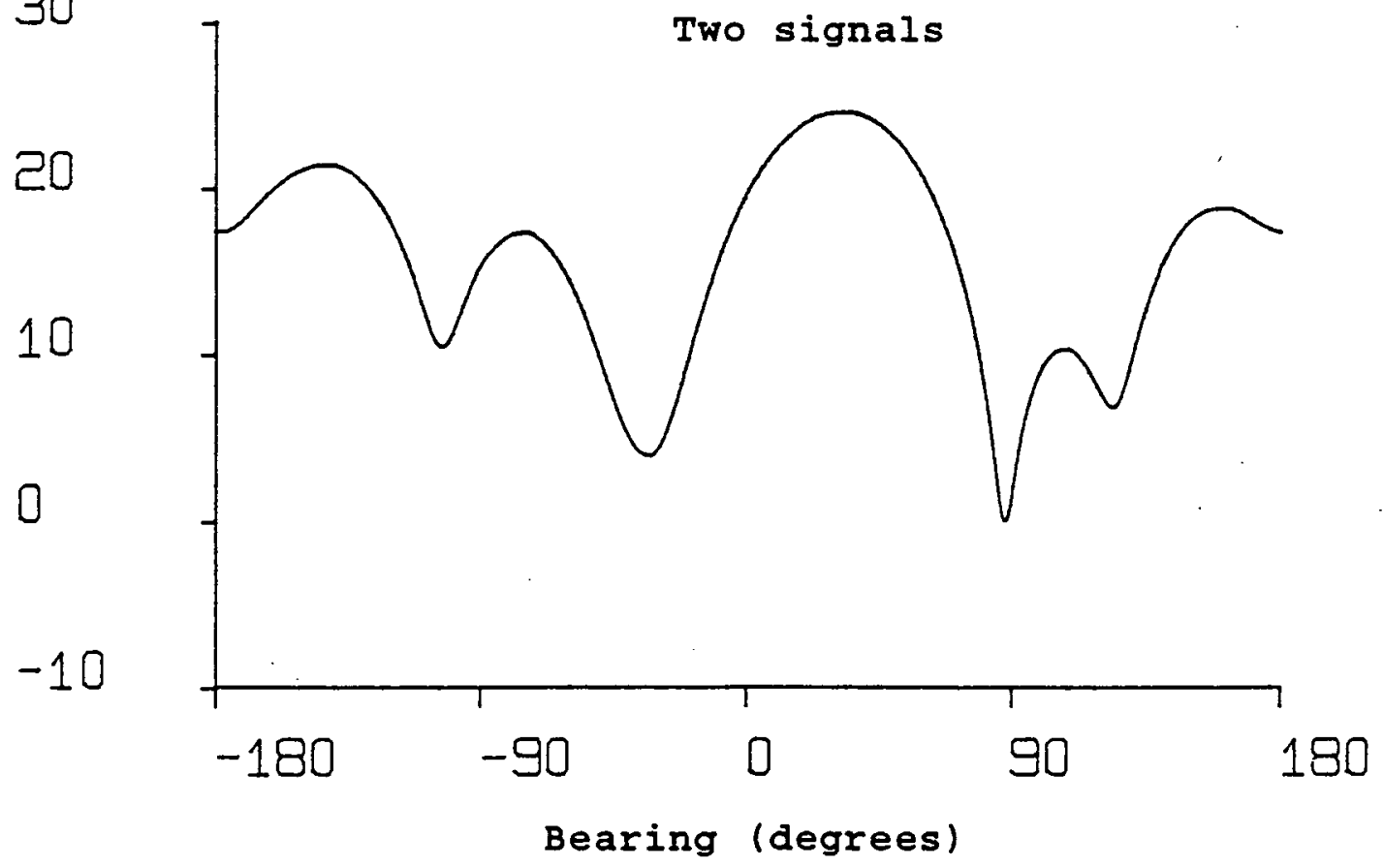

Figure 2.8 Conventional beamforming bearing spectra 

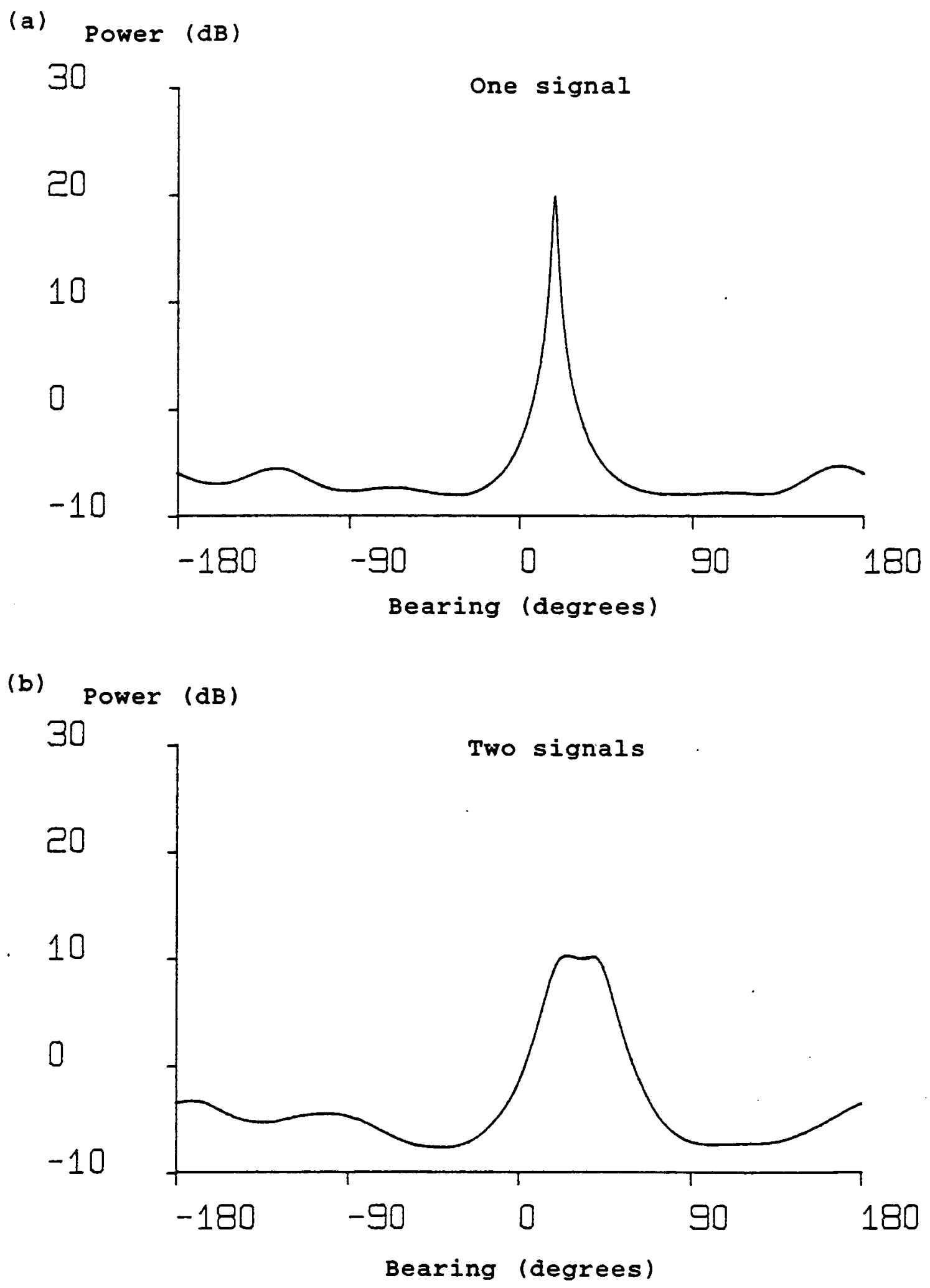

Figure 2.9 Minimum energy bearing spectra 
(a) $10 \log _{10} P_{\text {mu }}(\theta)$

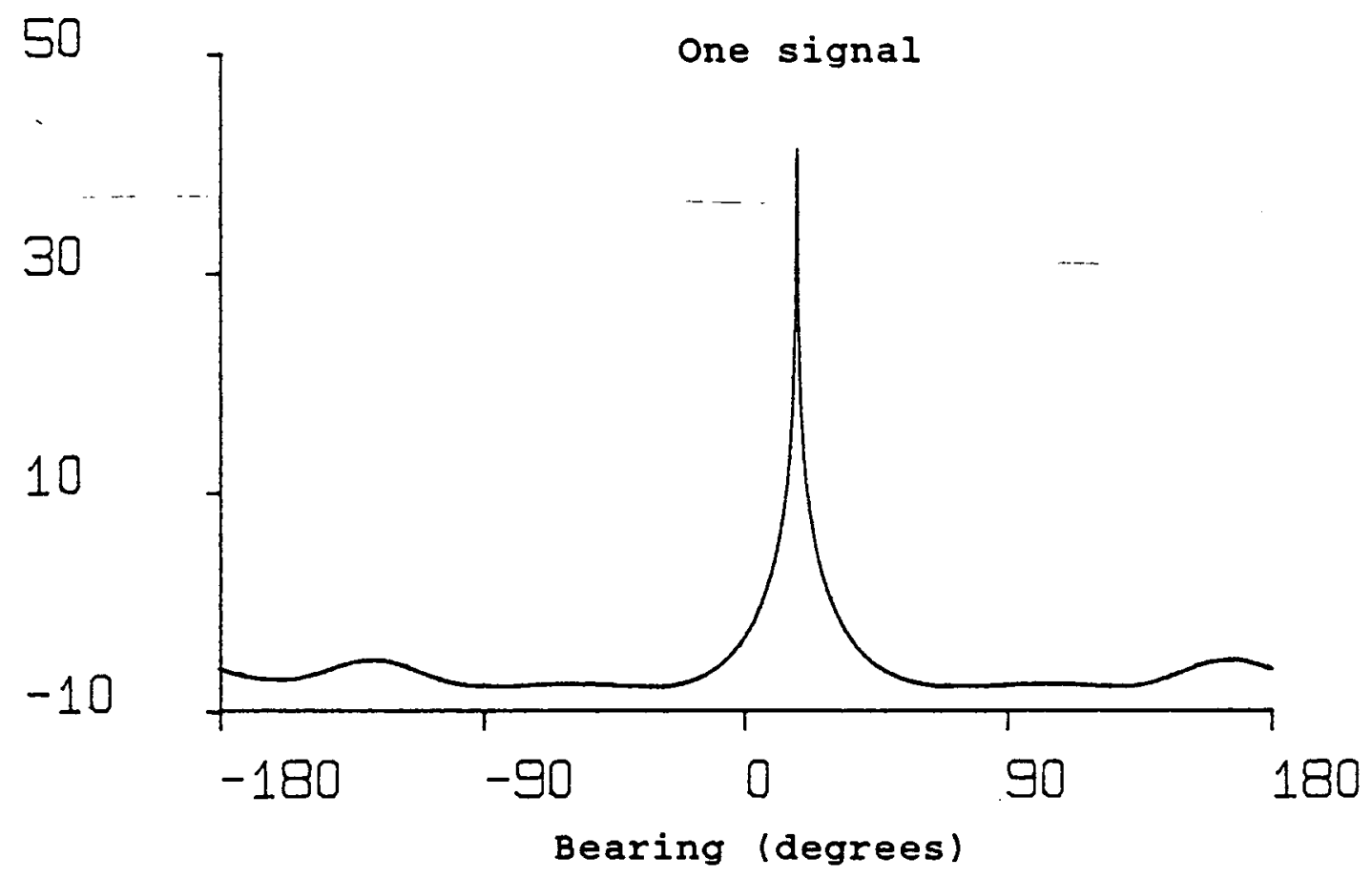

(b) $10 \log _{10} P_{\mathrm{mu}}(\theta)$

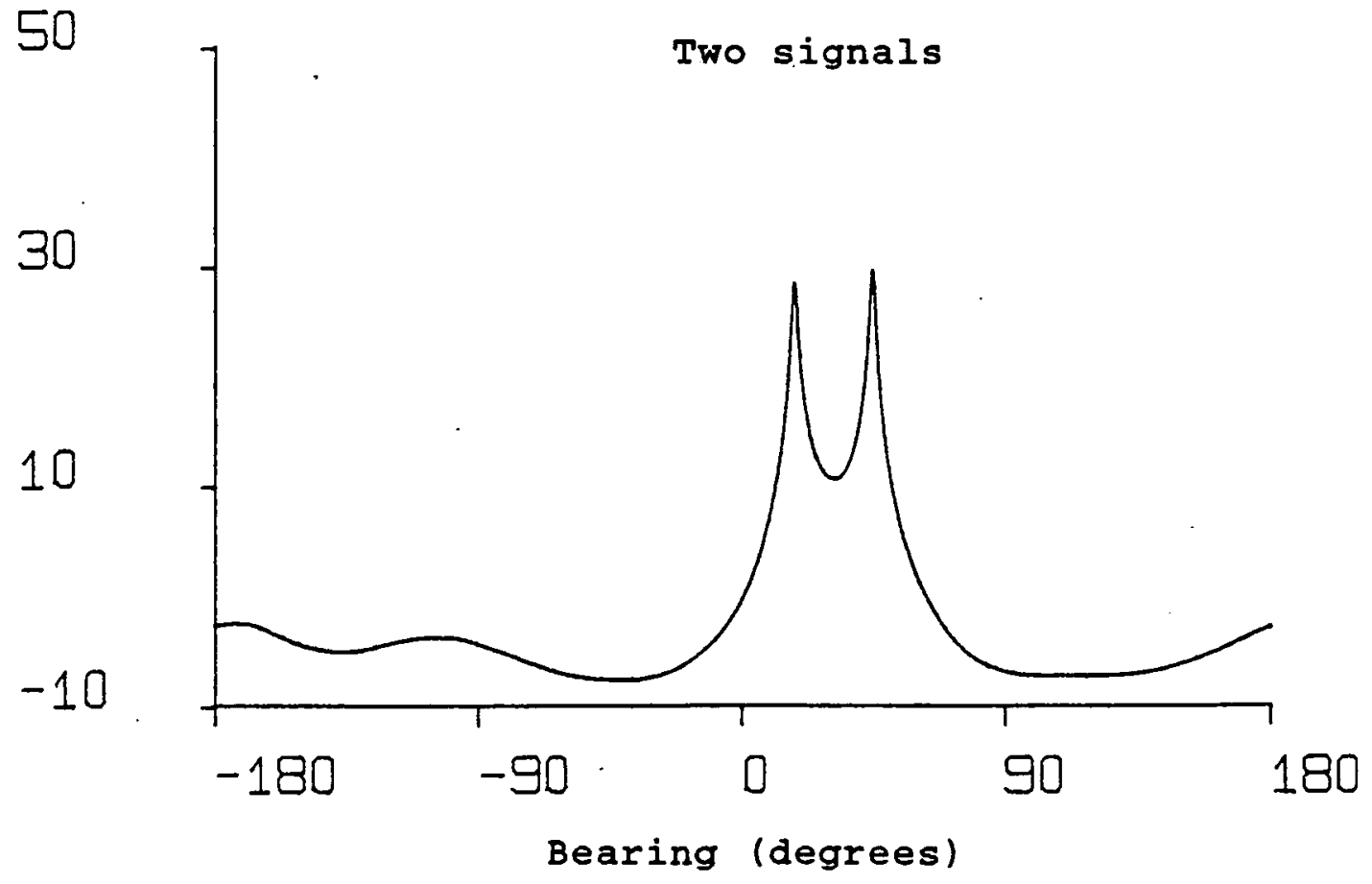

Figure 2.10 MUSIC bearing spectra 
spectrum, the peak height being theoretically equal to infinity. Thus the vertical axis of the MUSIC spectrum is merely $P_{m u}(\theta)$ plotted on a logarithmic scale. Marple [17] provides a detailed discussion of the units produced by various spectral estimators.

Figures $2.8(\mathrm{~b}), 2.9(\mathrm{~b})$, and $2.10(\mathrm{~b})$ show the bearing spectra obtained when a second signal with a bearing of 45 degrees was introduced. The second signal had a power $A_{2}$ of 20dB, a phase angle $\alpha_{2}$ of zero degrees, and a relative frequency $\omega_{2} / \omega_{c}$ of 1.001 . The noise and sampling parameters were as before. Notice that the MUSIC spectrum is the only one which contains two distinct peaks corresponding to the signals being received. This confirms the superior resolution properties of the MUSIC technique compared with conventional beamforming and minimum energy [16], [25].

As noted earlier, these results are not original and are included only to illustrate the output from the simulation system. The application of the software to original work is considered in Chapters 3 and 4 . 


\section{$\underline{2.17 \text { Summary }}$}

This chapter has described a number of techniques for performing bearing estimation using an array of sensors. The discussion started by developing a model for the snapshots obtained from the array, the main aim being to show how the bearings of the signals were embedded in the data.

The procedure for forming a covariance matrix from the snapshots was outlined and a number of properties of this matrix were derived, particular attention being given to its eigenvalues and eigenvectors. This led naturally to a discussion of signal and noise subspaces and a derivation of MUSIC, which is a bearing estimation algorithm based on subspace concepts.

This was followed by a description of the conventional beamforming approach to bearing estimation, which is essentially a spatial Fourier transform. The method is generally inferior to MUSIC, although it has the advantage of being less computationally expensive.

The minimum energy method was also described. This is a bearing estimation technique which attempts to improve the bearing spectrum by minimizing the received power subject to the constraint that the gain in the direction of 
interest is unity. The result is that the influence of signals from other directions is reduced. The technique lies somewhere between conventional beamforming and MUSIC in both performance and computational cost.

Finally, a number of bearing spectra produced by a computer simulation system were presented. These compared the three techniques and demonstrated the superior resolution properties of the MUSIC algorithm. 


\section{CHAPTER 3 THE EFFECTS OF SENSOR POSITIONING ERRORS}

\subsection{Introduction}

setting up a direction finding array involves attempting to place each sensor at its intended position. In practice, it is impossible to do this with total accuracy and there are always small errors between the intended sensor positions and the true ones.

This chapter presents an original piece of research which investigates the effects of such errors on the bearing estimation process. This work is a natural extension to the background material presented in Chapter 2.

The analysis is based on a statistical model of the sensor positioning errors. Imagine that an array of sensors is set up several times and that the errors in the $X$ and $Y$ coordinates of the sensors are measured on each occasion. It is assumed that the error in each coordinate would be found to have a normal distribution with a mean of zero. Furthermore, all of the errors would have the same variance, denoted by $\sigma_{p}^{2}$. This variance expresses the accuracy to which the sensors can be positioned. 
The errors are therefore random variables and a particular attempt at setting up the array is represented by a set of samples of these variables. Sensor positioning errors are essentially another noise source associated with each sensor although they are different from receiver noise in that they are sampled only when the array is set up instead of once every snapshot.

Figure 3.1 shows two sets of sensor positions for a linear array. The intended sensor positions define the ideal shape of the array while the true sensor positions show the result of one particular attempt at setting the array up. The true positions incorporate the sensor positioning errors.

Although the snapshots from the array depend on the true sensor positions, the bearing estimation algorithm used to analyze the snapshots is normally provided with signal vectors based on the intended positions. This presents no problems under the usual tacit assumption that the two sets of positions are identical. However, when the presence of sensor positioning errors is acknowledged, it becomes evident that the data model assumed by the bearing estimation algorithm does not match the one which generated the snapshots. 
0

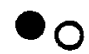

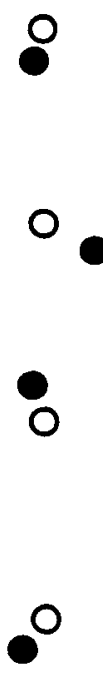

O intended sensor positions

- true sensor positions

Figure 3.1 True and intended sensor positions 
This chapter shows that the bearing spectrum is adversely affected if there are inconsistencies between the true sensor positions and the ones used for bearing estimation. Sensor positioning errors cause errors in the bearings of the peaks in the spectrum and a reduction in peak height.

The imaginary experiment introduced at the start of this section provides a good framework within which to describe the goals of the work presented in this chapter. In the experiment, an array of sensors is set up several times, producing a new set of true sensor positions on each occasion. For each set of true positions, a sequence of snapshots is obtained and a bearing spectrum is formed using using one of the bearing estimation techniques described in Chapter 2 . The signal vectors $\underset{\sim}{\hat{q}}(\theta)$ required by the bearing estimation algorithm are derived from the intended sensor positions and are the same on each occasion. The resulting spectrum is examined and values are noted for certain parameters, such as the height of a peak or the error between the bearing of the peak and the true bearing of the corresponding signal.

When several sets of true sensor positions have been processed in this way, a mean and variance can be calculated for each of the parameters and these means and variances can be viewed as functions of the variance of 
the sensor positions, $\sigma_{p}^{2}$. For example, if the experiment was repeated with a larger value of $\sigma_{p}^{2}$, there would generally be larger errors in the bearing spectrum. Thus the results are statistical in nature rather than being restricted to a particular realization of the sensor positioning errors.

The layout of the chapter is as follows. The next section introduces the new notation required to describe sensor positioning errors. Following this, the relevant parameters of the bearing spectrum are identified. Finally, expressions are derived which relate the mean and variance of each parameter to $\sigma_{p}^{2}$ and the results are confirmed using computer simulations.

\subsection{Modelling of Sensor Positioning Errors}

The main purpose of this section is to establish a frame of reference within which sensor positioning errors can be described. It is important to realize that the degraded performance of a bearing estimation algorithm in the presence of such errors is caused not by the errors per se but by the mismatch created when the errors are not taken into account. 
When performing bearing estimation, the fact that the intended sensor positions are the ideal values of the true ones is a philosophical point with no physical significance. From a practical standpoint, the intended positions are merely estimates of the true ones.

Indeed, this concept is taken to its natural conclusion in Chapter 4 by the array calibration algorithm, which updates the estimated sensor positions iteratively in an attempt to make them converge to the true ones. The intended positions are the initial estimates. After calibration, the estimated positions are used in place of the intended ones when performing bearing estimation, thus removing (or reducing) the mismatch.

The vector $\hat{u}_{n}$ introduced in Chapter 2 represents the intended position of sensor $n$. The error between the intended position and the true one is denoted by $\Delta \underset{\sim}{\mathrm{n}}$, as shown in Figure 3.2. The $X$ and $Y$ components of the $N$ vectors $\Delta u_{n}$ are assumed to be independent random variables, each of which has a normal distribution with a mean of zero and a variance of $\sigma_{p}^{2}$.

Since the $X$ and $Y$ components of $\Delta{\underset{\sim}{n}}_{n}$ have identical normal distributions, errors in all directions are equally likely to occur. It also follows that the length $\| \Delta_{\sim} u_{n}$ has a Rayleigh distribution [97]. 
Y (wavelengths)

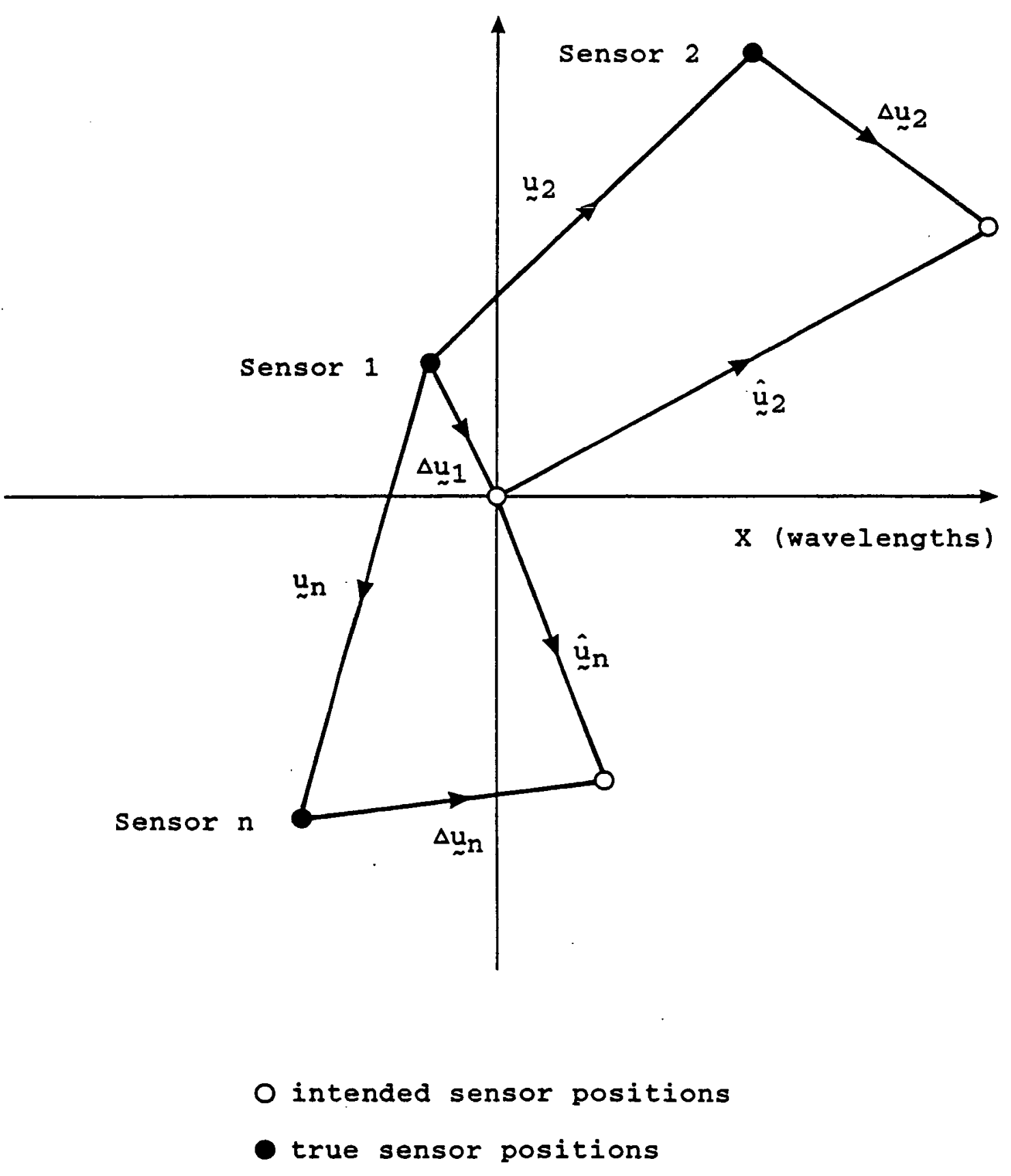

Figure 3.2 Vectors for analysis of sensor positioning errors 
The true position of sensor $n$ relative to the true position of the reference sensor (sensor 1) is denoted by the vector $u_{n}$, which is defined as follows.

$$
\underline{\sim}_{\mathrm{n}}=\hat{u}_{\mathrm{n}}-\Delta \underline{\sim}_{\mathrm{n}}+\Delta \underline{\sim}_{1}
$$

By setting $n$ to 1 in (3.1), it is straightforward to show that ${ }_{\sim}$, like $\hat{u}_{1}$, is always a null vector.

$$
\underline{\sim}_{1}=\hat{u}_{1}=\underline{\sim}
$$

This is important because the displacement of the reference sensor from the origin in Figure 3.2 implies a translation of the whole array. However, the phase information obtained from the array depends only on the relative positions of the sensors and a translation of the array is therefore not observable from the snapshots. It is assumed that the sensor positioning errors are small enough for the plane wave assumption to remain valid over the entire area covered by both the true and intended positions.

Figure 3.3 shows an alternative view of the array in which the true and intended positions of the reference sensor are both at the origin. The absolute error in the true position of sensor 1 has been absorbed by the other sensors. In a sense, $\sigma_{p}^{2}$ has been set to zero for the 
Y (wavelengths)

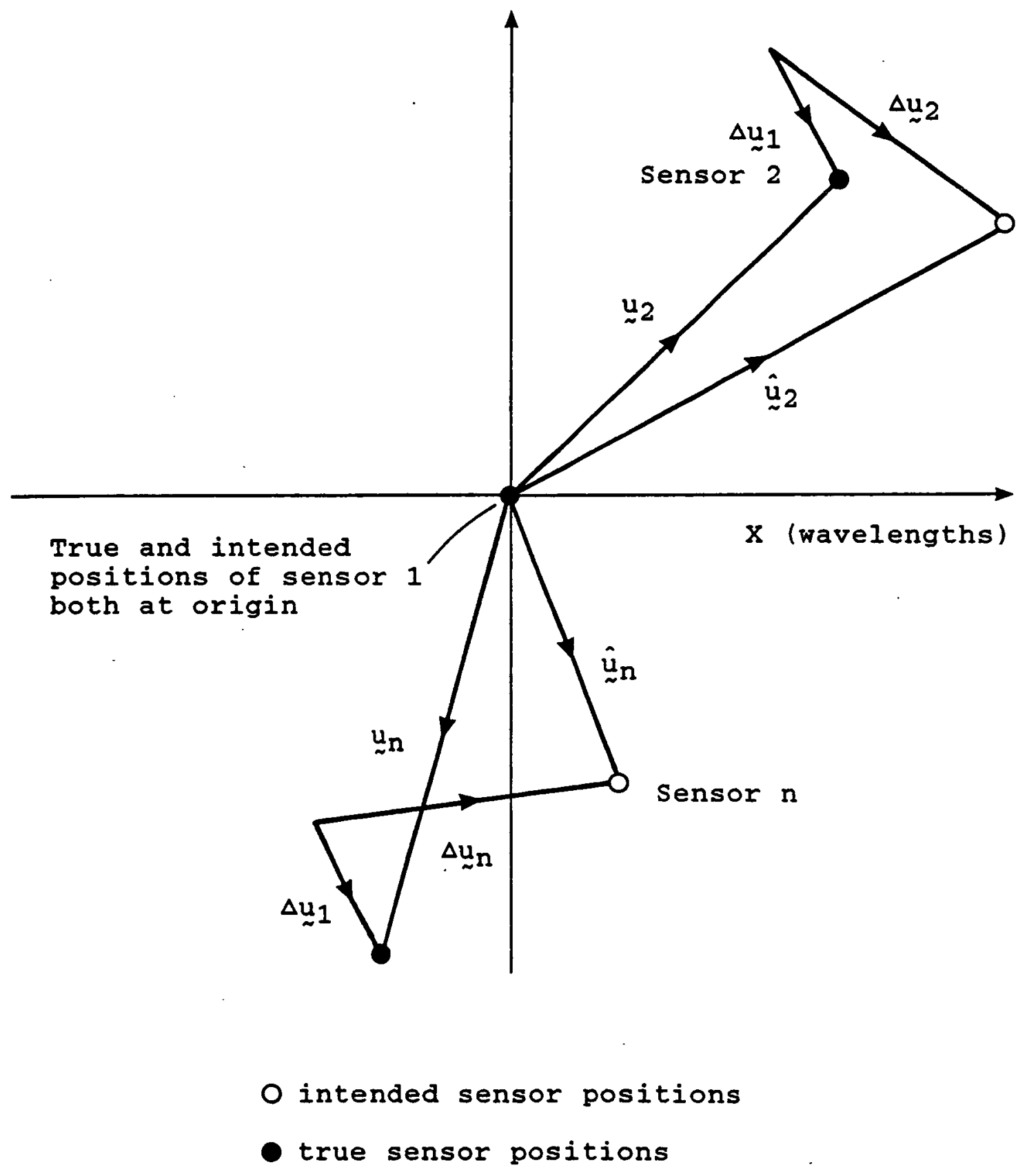

Figure 3.3 Alternative view of vectors for analysis of sensor positioning errors 
reference sensor and doubled for all of the others.

The lengths of the components of $\Delta_{\sim} u_{n}$ and ${\underset{\sim}{n}}_{n}$ along the axis of propagation of signal $\mathrm{m}$ are denoted by $\Delta \epsilon_{\mathrm{nm}}$ and $\epsilon_{\mathrm{nm}}$ respectively. They are calculated in the same way as $\hat{\epsilon}_{\mathrm{nm}}$.

$$
\begin{aligned}
& \Delta \epsilon_{\mathrm{nm}}=\Delta \underset{\sim}{u_{n}} \cdot{\underset{\sim}{z} \mathrm{~m}} \\
& \epsilon \mathrm{nm}={\underset{\sim}{\mathrm{n}}}_{\mathrm{n}} \cdot{\underset{\sim}{\mathrm{z}} \mathrm{m}}
\end{aligned}
$$

Using the distributive property of the scalar product [100], the relationship between $\epsilon_{\mathrm{nm}}, \Delta \epsilon_{\mathrm{nm}}$, and $\hat{\epsilon}_{\mathrm{nm}}$ can be obtained from (3.1).

$$
\epsilon_{\mathrm{nm}}=\hat{\epsilon}_{\mathrm{nm}}-\Delta \epsilon_{\mathrm{nm}}+\Delta \epsilon_{1 \mathrm{~m}}
$$

setting $n$ to 1 in $(3.5)$ confirms that $\epsilon_{1 \mathrm{~m}}$, like $\hat{\epsilon}_{1 \mathrm{~m}}$, is always zero.

$$
\epsilon_{1 \mathrm{~m}}=\hat{\epsilon}_{1 \mathrm{~m}}=0
$$

Figure $3.4(a)$ shows $\epsilon_{\mathrm{nm}}, \hat{\epsilon}_{\mathrm{nm}}, \Delta \epsilon_{\mathrm{nm}}$, and $\Delta \epsilon_{1 \mathrm{~m}}$ with the true position of the reference sensor displaced from the origin as in Figure 3.2. Figure $3.4(b)$ is based on the alternative construction from Figure 3.3 in which the true and intended positions of sensor 1 are both shown at the origin. Notice that $\epsilon_{\mathrm{nm}}, \hat{\epsilon}_{\mathrm{nm}}, \Delta \epsilon_{\mathrm{nm}}$, and $\Delta \epsilon_{1 \mathrm{~m}}$ are the same in both cases although the measurements are shown at 
(a)

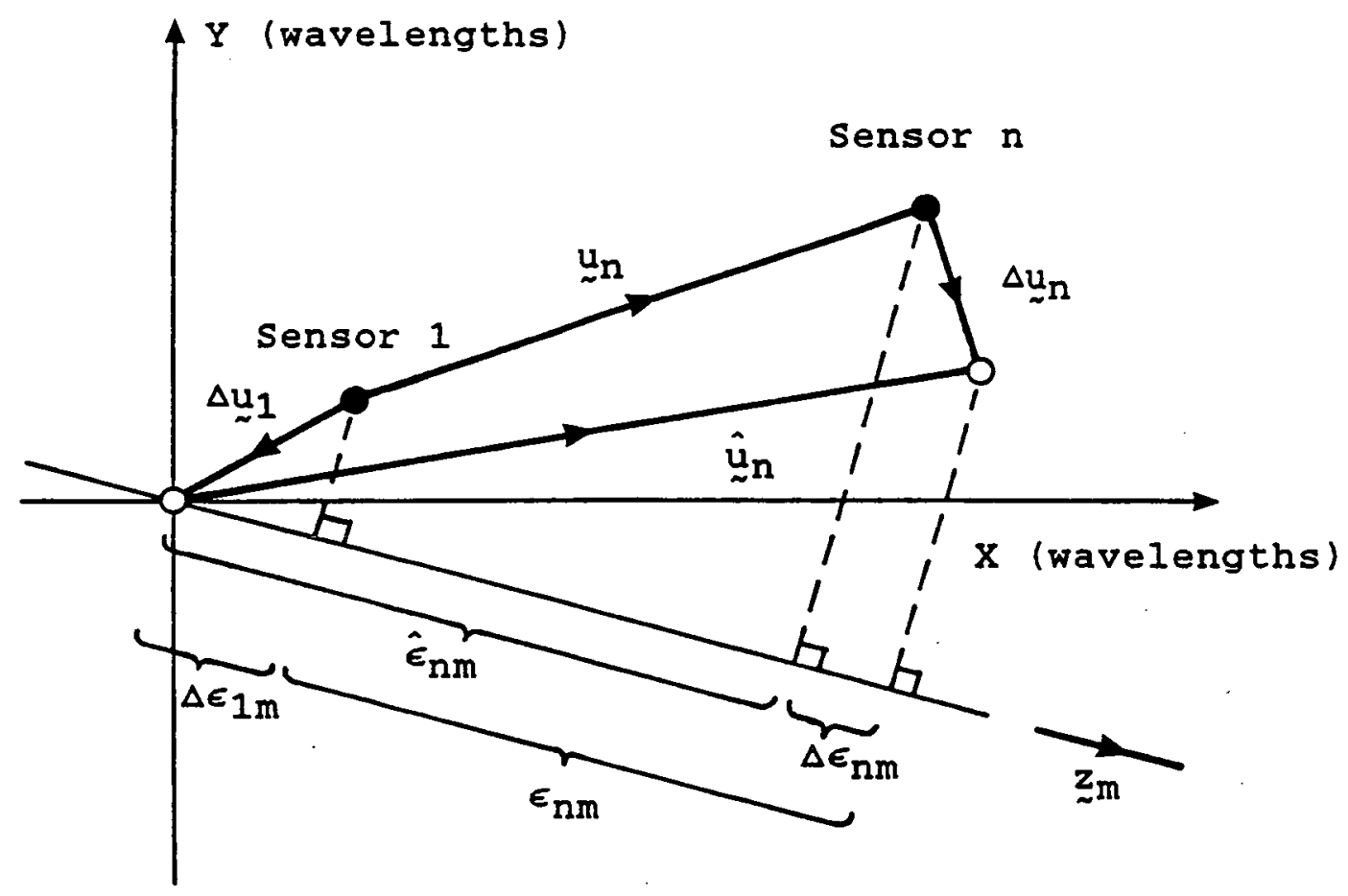

(b)

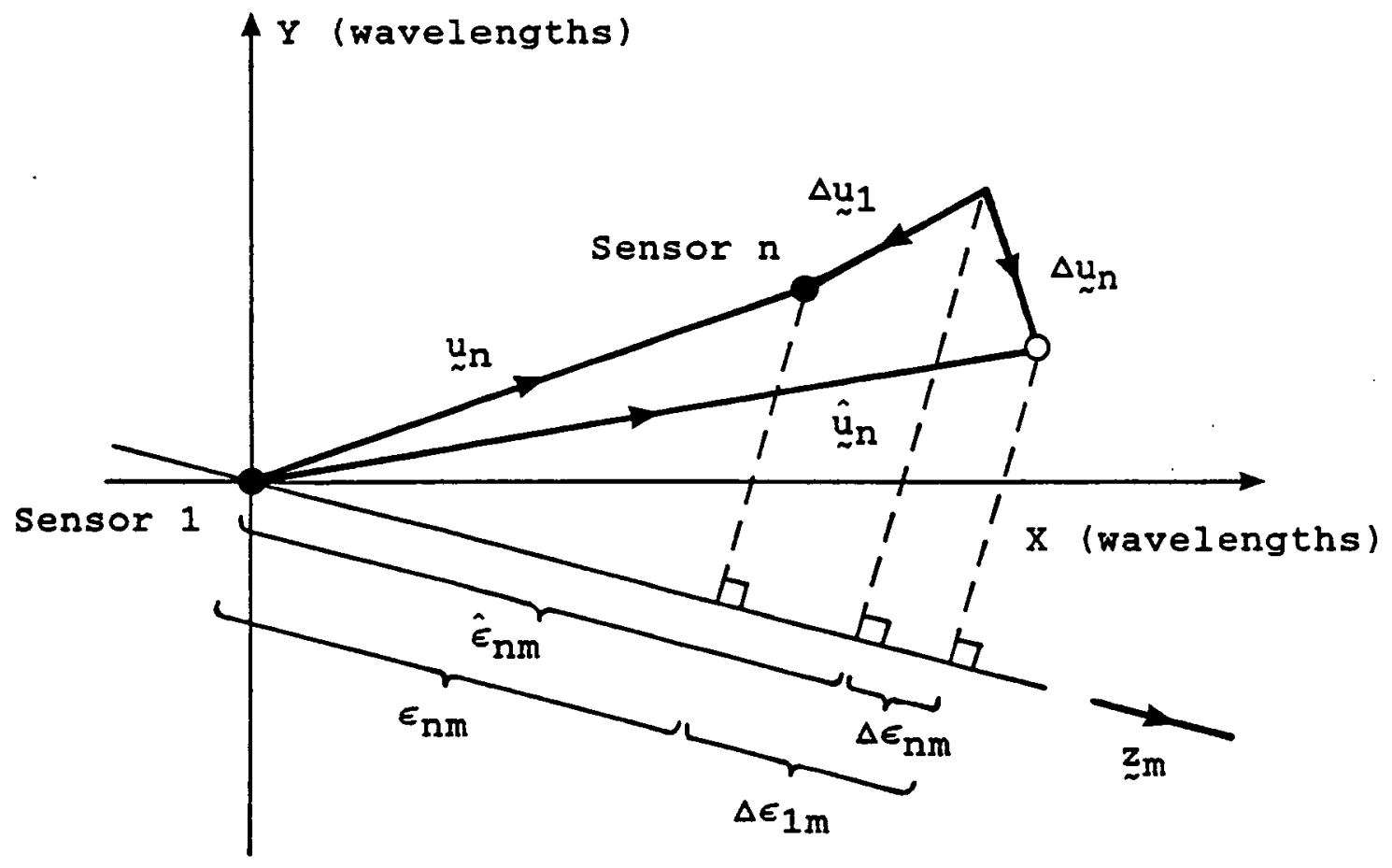

Figure 3.4 Distances for analysis of sensor positioning errors 
different positions along the axis of transmission.

Since the components of $\Delta_{\sim} u_{n}$ along the $X$ and $Y$ axes have the same distribution, the component along any axis must have that distribution as well. Thus $\Delta \epsilon_{\mathrm{nm}}$, the component of $\Delta u_{n}$ along the axis of transmission of signal $m$, has a normal distribution with a mean of zero and a variance of $\sigma_{\mathrm{p}}^{2}$

The probability that $\Delta \epsilon_{\mathrm{nm}}$ is equal to a particular value, say $P$, is obtained from the probability density function $g(\rho)$. Since $\Delta \epsilon_{n m}$ is normally distributed, $g(p)$ is as follows [97].

$$
g(\rho)=\frac{1}{\sigma_{p} \checkmark\langle 2 \pi\rangle} \exp \left\{-\frac{\rho^{2}}{2 \sigma_{p}^{2}}\right\},-\infty<\rho<\infty
$$

\subsection{Parameters of the Bearing spectrum}

The last section showed that the errors $\Delta \epsilon_{\mathrm{nm}}$ in the distances $\hat{\epsilon}_{\mathrm{nm}}$ could be characterized statistically by the variance $\sigma_{p}^{2}$. The next step is to consider the effects of these errors on the bearing spectrum itself. 
This section introduces a small set of parameters which can be used to describe a bearing spectrum generated in the presence of sensor positioning errors. Later sections relate the mean and variance of each parameter to $\sigma_{\mathrm{p}}^{2}$

The analysis is restricted to the case where only one signal (plus sensor noise) is being received. Under these conditions, all of the bearing estimation techniques introduced in Chapter 2 are asymptotically unbiased [12], [16], [24]. This means that the bearing spectrum would have a peak at the correct bearing if the expected value of the covariance matrix was used and there were no sensor positioning errors.

When there are errors in the sensor positions, the peak is generally at the wrong bearing and the overall shape of the spectrum is changed slightly. Figure 3.5 shows the parameters used to describe these effects. The angle $\Delta \theta_{1}$ is the error in radians between the true bearing $\theta_{1}$ of the signal and the bearing $\theta_{1}+\Delta \theta_{1}$ of the peak in the spectrum. $P\left(\theta_{1}+\Delta \theta_{1}\right)$ is the height of the peak and $P\left(\theta_{1}\right)$ is the height of the spectrum at the true bearing of the signal.

Later sections of this chapter derive theoretical expressions for the means and variances of $\Delta \theta_{1}$ and $P\left(\theta_{1}\right)$ 


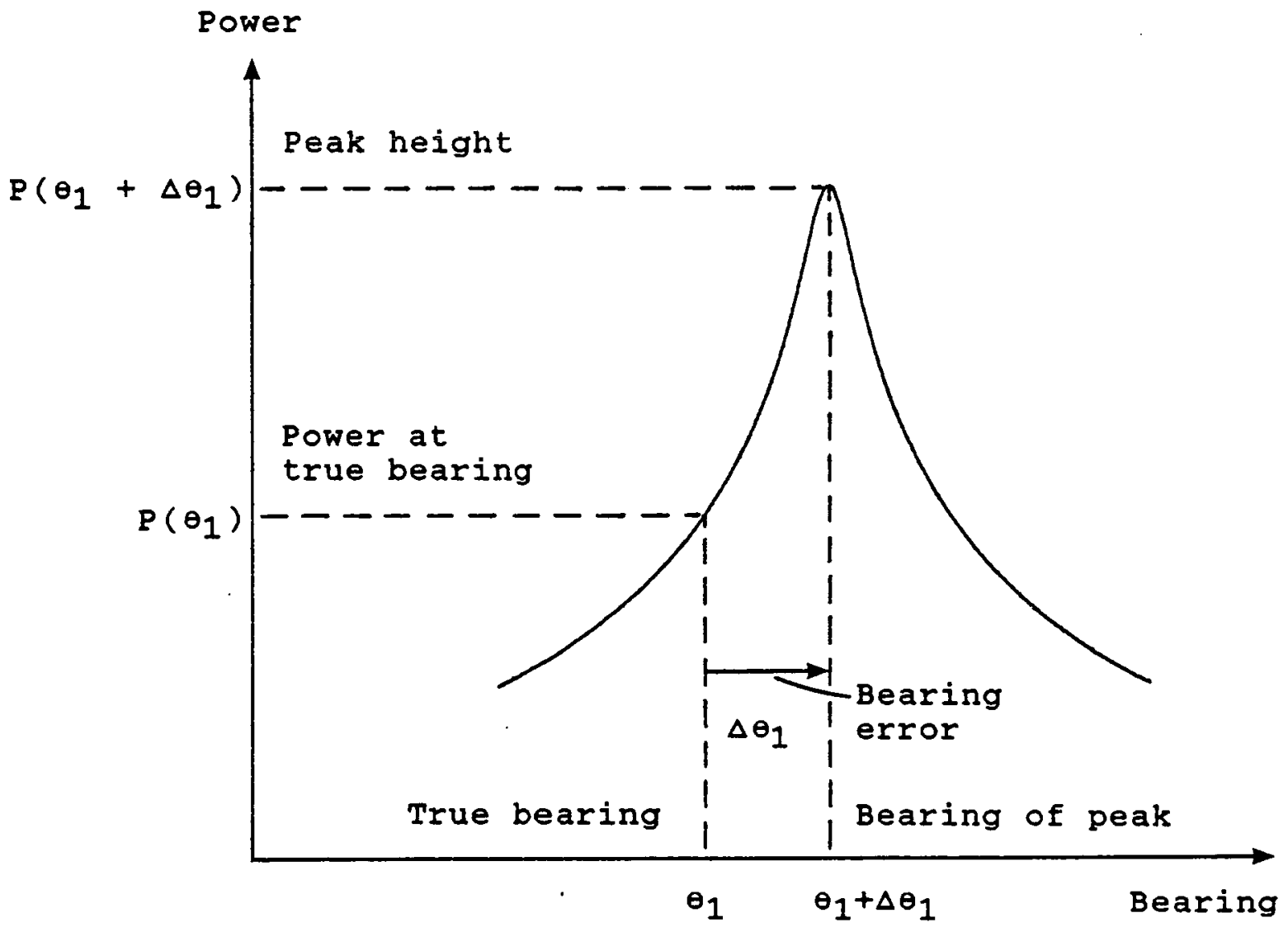

Figure 3.5 Parameters of bearing spectrum 
as functions of $\sigma_{p}^{2}$. The results obtained for $\Delta \theta_{1}$ apply to all of the bearing estimation techniques introduced in Chapter 2, while in the case of $P\left(\theta_{1}\right)$, different expressions are produced for the MUSIC, conventional .. beamforming, and minimum energy methods.

Computer simulations confirm these results and also record the behaviour of the peak height $P\left(\theta_{1}+\Delta \theta_{1}\right)$, which is arguably of more interest than $P\left(\theta_{1}\right)$. Although no theoretical expressions are obtained for the peak height, it is evident from the simulation results that the expressions for $P\left(\theta_{1}\right)$ can be used to construct bounds for $P\left(\theta_{1}+\Delta \theta_{1}\right)$.

\subsection{Computer simulation}

The simulation system includes a program which models the process of setting up an array of sensors several times. The inputs to the program are the intended sensor positions $\hat{u}_{n}$, the variance $\sigma_{p}^{2}$, and a number which specifies how many times the array is to be set up. The output from the program consists of several sets of true sensor positions ${ }_{\sim}$. 
The program simulates the act of setting up the array by creating the sensor positioning errors $\Delta \mathrm{\sim}_{\mathrm{n}}$ using the random number generator. Recall that the $\mathrm{X}$ and $\mathrm{Y}$ components of $\Delta u_{n}$ both have a normal distribution with a mean of zero and a variance of $\sigma_{p}^{2}$. The program can therefore create the sensor positioning error $\Delta \mathrm{\sim}_{\mathrm{n}}$ by generating a random complex number using the procedure described in Chapter 2 then assigning the real and imaginary parts of the complex number to the $X$ and $Y$ components of $\Delta_{\sim}$. The true sensor positions $u_{n}$ are then produced by combining the intended positions $\hat{u}_{n}$ with the errors $\Delta u_{n}$ using $(3.1)$.

For each set of true sensor positions ${\underset{\sim}{n}}_{n}$, a covariance matrix $\underset{\sim}{R}$ was formed by processing the sensor positions together with a set of signal parameters as described in Chapter 2. Each covariance matrix was then fed to the bearing estimation software and bearing spectra were produced using the conventional beamforming, minimum energy, and MUSIC techniques. However, the signal vectors $\hat{q}(\theta)$ which appear in the expressions for $P_{b f}(\theta)$, $\mathrm{P}_{\mathrm{me}}(\theta)$, and $\mathrm{P}_{\mathrm{mu}}(\theta)$ were derived from the intended sensor positions rather than the true ones used in forming the covariance matrix. As a result, the bearing spectra showed the effects of the inconsistencies between the intended sensor positions and the true ones. 
All of the simulation results in this chapter are based on the circular array introduced in Chapter 2. This array defines the intended sensor positions. As an example, Figure 3.6 shows the result of generating 5 sets of true sensor positions (and hence 5 bearing spectra) with $\sigma_{\mathrm{p}}^{2}$ set to 0.001 . The bearing spectra were produced using MUSIC. In Figure 3.6(a) there is one signal present, while in Figure $3.5(b)$ the whole process has been repeated using two signals. The signal and sampling parameters were identical to those used in Chapter 2 and Figure 3.6 may therefore be compared directly with Figure 2.10 .

To recap, the first signal had a bearing of 18 degrees and a relative frequency of 1.0 while the second signal had a bearing of 45 degrees and a relative frequency of 1.001 . Both signals had a power of $20 \mathrm{db}$ and an initial phase angle of zero degrees. The noise power was 0 dB and the covariance matrix was formed using 500 snapshots obtained at a sampling rate of 3.4567 samples per cycle.

Comparing Figure 3.6 with Figure 2.10, it is evident that the sensor positioning errors caused variations in the bearings of the peaks in the spectra and a general reduction in peak height. In Figure $3.6(b)$, notice how the errors considerably reduced the ability of the MUSIC technique to resolve the two signals. 
(a) $10 \log _{10} \mathrm{P}_{\mathrm{mu}}(\theta)$

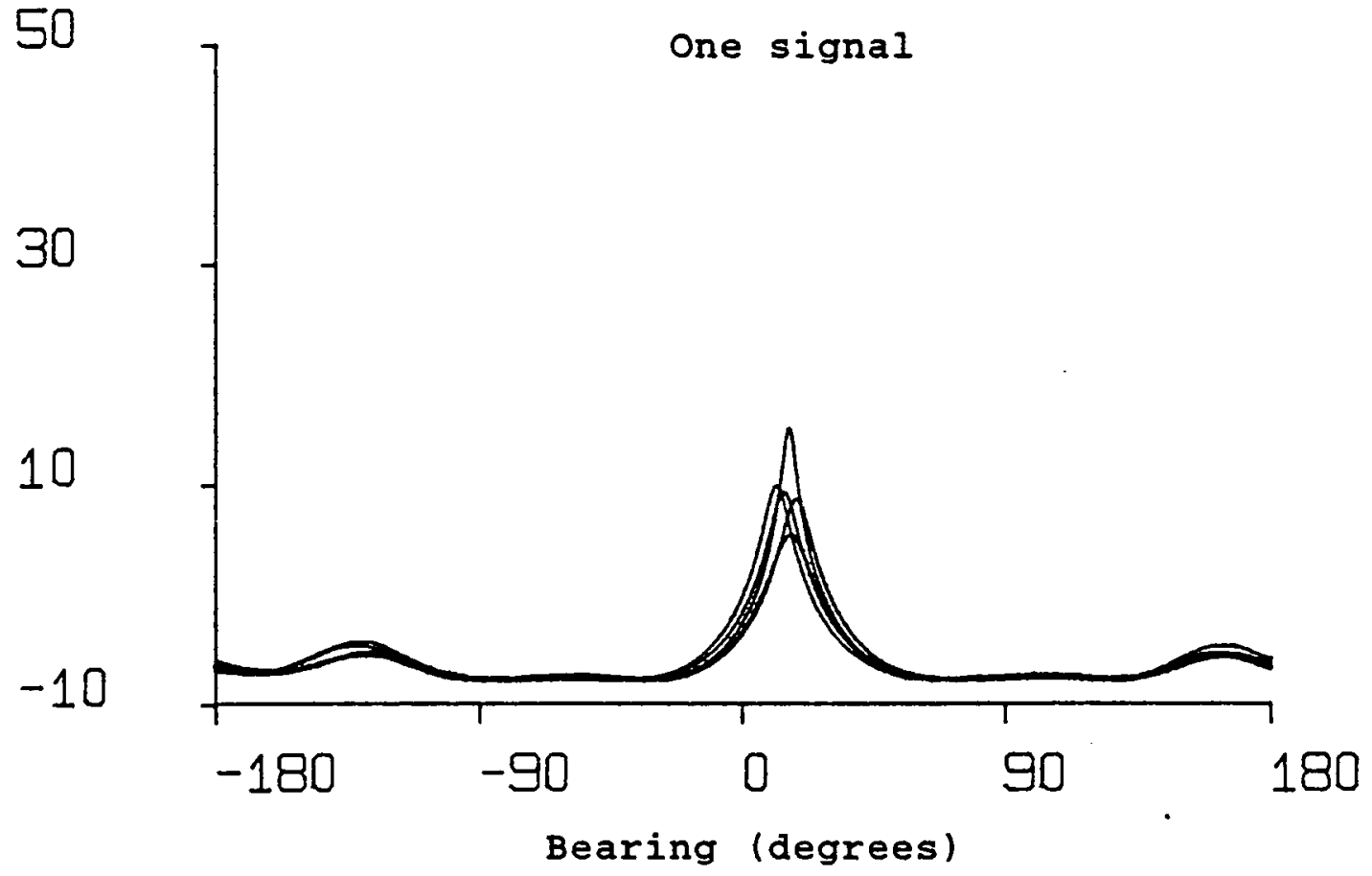

(b) $10 \log _{10} P_{\mathrm{mu}}(\theta)$

50

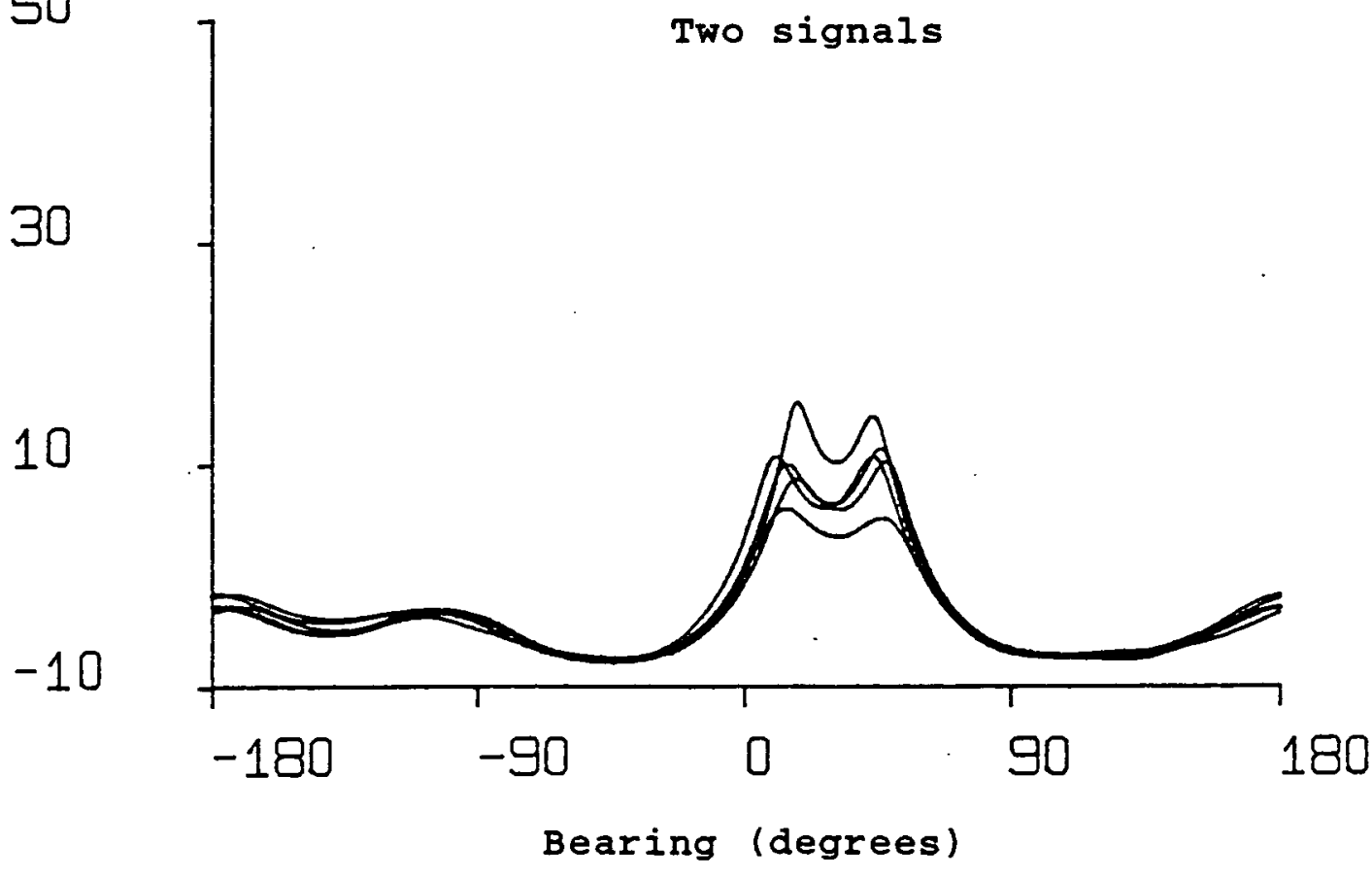

Figure 3.6 MUSIC bearing spectra with sensor positioning errors 
Although Figure 3.6 is useful as an illustration of the effects of sensor positioning errors, a sample of 5 true arrays is too small to allow reliable statistical results to be obtained. The main simulation results for this chapter are based on an experiment in which 1000 sets of true sensor positions were generated for each value of $\sigma_{\mathrm{p}}^{z}$.

As noted earlier, the theoretical analysis is restricted to the case where only one signal is present. In the simulation, the bearing of this signal was 18 degrees, its power was $20 \mathrm{~dB}$, its relative frequency was 1.0 , and its initial phase angle was zero degrees. The noise power was $O d B$ (resulting in a signal to noise ratio of $20 \mathrm{~dB}$ ).

For each of the 1000 sets of true sensor positions, the expected value of the covariance matrix was obtained using the procedure described in chapter 2. The theoretical work does not take account of the effects of averaging over a finite number of snapshots and the use of the expected value of the covariance matrix allowed such effects to be excluded from the simulation results as well.

For each of the 1000 covariance matrices, bearing spectra were produced using the conventional beamforming, minimum energy, and MUSIC techniques. The spacing between adjacent points was 0.005 degrees. Rather than being plotted, however, these spectra were fed into a program 
which gathers statistics on the parameters introduced in the last section.

The program searches through each spectrum to find the peak then calculates the bearing error $\Delta \theta_{1}$, the peak height $P\left(\theta_{1}+\Delta \theta_{1}\right)$, and the height $P\left(\theta_{1}\right)$ of the spectrum at the true bearing of the signal. The program calculates the mean and variance of each of these parameters over the 1000 spectra.

Since there were three bearing estimation techniques, three parameters for each technique, and a mean and variance for each parameter, the program produced a total of 18 results. The final step in the simulation was to plot these 18 results against the variance of the sensor positioning errors $\sigma_{p}^{2}$. In the graphs shown later in the chapter, $\sigma_{p}^{2}$ runs from 0 to 0.001 in steps of 0.0001 , producing 11 values in total. For each value of $\sigma_{p}^{2}, 1000$ sets of true sensor positions were produced and analyzed in the manner just described.

A further program was used to plot the theoretical results over the same range of values of $\sigma_{p}^{2}$. Each of the 18 graphs consists of a continuous theoretical curve and the 11 data points. These graphs are presented in later sections at the points where the corresponding theoretical results are derived. 


\subsection{A Geometric View of Bearing Estimation}

The first step in understanding the behaviour of the bearing error $\Delta \theta_{1}$ is to develop a geometric interpretation of the bearing estimation process. Although bearing estimation algorithms are normally expressed in terms of phasors and matrix algebra, a much simpler analysis involving only distances and angles can be used to anticipate the bearing of the peak in the spectrum. This simplified view makes it possible to relate the mean and variance of the bearing error $\Delta \theta_{1}$ to the sensor positioning errors and hence to $\sigma_{\mathrm{p}}^{2}$.

In Eigure 3.7 , sensors $h$ and $i$ are receiving one signal with a bearing of $\theta_{1}$ radians. The indices $h$ and $i$ both lie in the range 1 to $\mathrm{N}$ and $\mathrm{h} \neq \mathrm{i}$. The diagram shows the general case where neither of the two sensors is the reference sensor (sensor 1) although all of the relationships derived in the following discussion apply equally well to cases where one of the sensors is the reference sensor.

The vector difference $\hat{u}_{h}-\hat{u}_{i}$ expresses the displacement from the intended position of serisor $i$ to that of sensor $h$. The length of the component of $\hat{u}_{h}-\hat{\sim}_{i}$ along the axis of propagation is $\hat{\epsilon}_{\mathrm{h} 1}-\hat{\epsilon}_{i 1}$ as illustrated and this distance provides the link between the sensor positions 


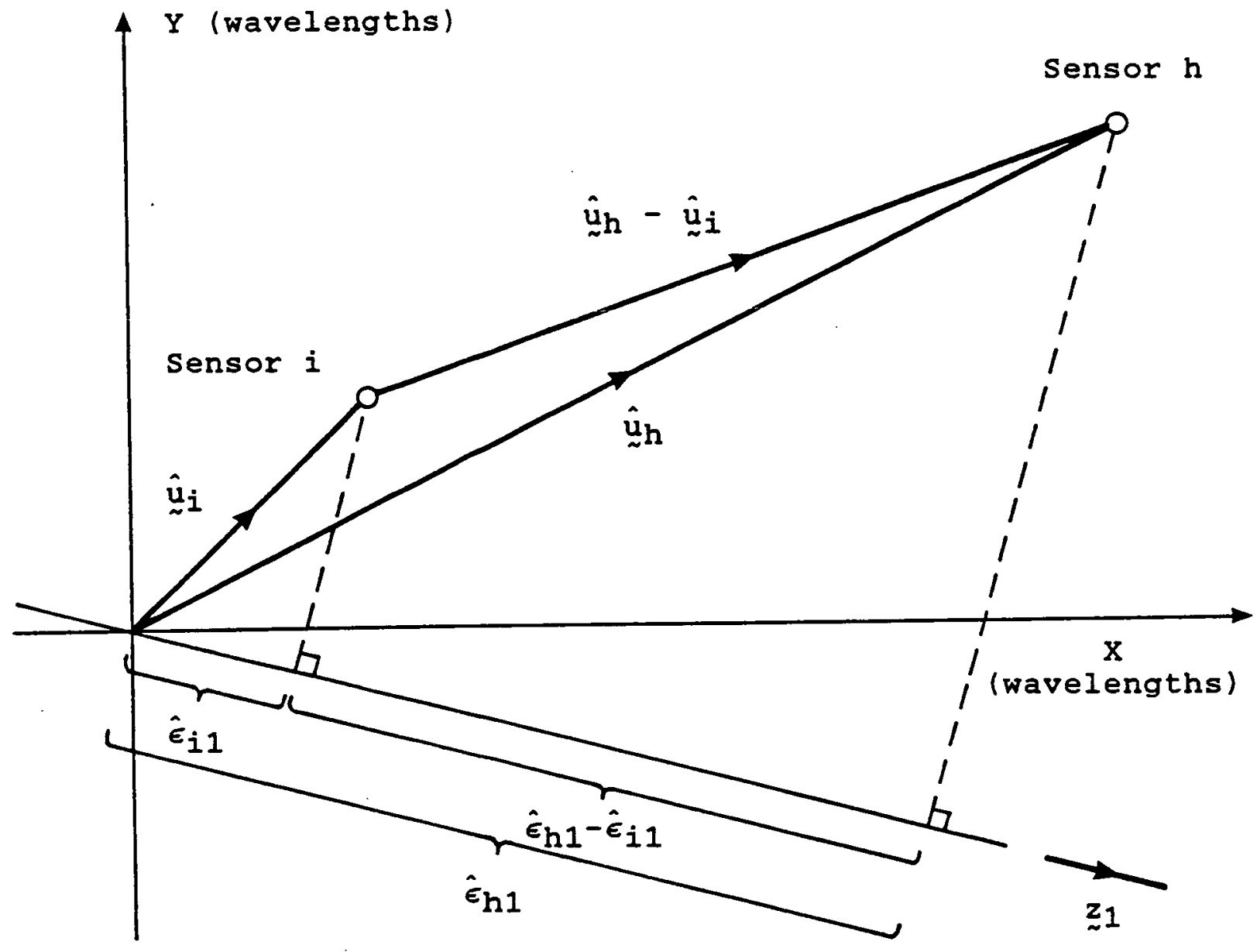

Figure 3.7 Distances for intended positions of sensors $h$ and $i$ 
and the bearing of the signal.

$$
\left(\hat{u}_{h}-\hat{u}_{i}\right) \cdot z_{1}=\hat{\epsilon}_{h 1}-\hat{\epsilon}_{i 1}
$$

However, the sampling process produces information about phase differences rather than direct measurements of distance. From Chapter 2, the phase shifts from the reference sensor to sensors $\mathrm{h}$ and $i$ are $\hat{\psi}_{\mathrm{h} 1}=2 \pi \hat{\epsilon}_{\mathrm{h} 1}$ and $\hat{\psi}_{i 1}=2 \pi \hat{\epsilon}_{i 1}$, which are represented by the phasors $\hat{q}_{h 1}=\exp \left\langle j \hat{\psi}_{h 1}\right\rangle$ and $\hat{q}_{i 1}=\exp \left\langle j \hat{\psi}_{i 1}\right\}$. The phase difference between sensors $h$ and $i$ is therefore as follows.

$$
\hat{\psi}_{\mathrm{h} 1}-\hat{\psi}_{\mathrm{i} 1}=2 \pi\left(\hat{\epsilon}_{\mathrm{h} 1}-\hat{\epsilon}_{\mathrm{i} 1}\right)
$$

The angle $\hat{\Psi}_{\mathrm{h} 1}-\hat{\Psi}_{\mathrm{i} 1}$ is represented by the phasor $\hat{\mathrm{q}}_{\mathrm{h} 1} / \hat{\mathrm{q}}_{\mathrm{i} 1}$.

$$
\frac{\hat{q}_{h 1}}{\hat{q}_{i 1}}=\frac{\exp \left\langle j \hat{\psi}_{h 1}\right\}}{\exp \left\langle j \hat{\psi}_{i 1}\right\rangle}=\exp \left\{j\left(\hat{\psi}_{h 1}-\hat{\psi}_{i 1}\right)\right\}
$$

Notice that information may be destroyed when converting from a phase difference to a phasor since any complete cycles of $2 \pi$ radians are lost. If, for example, the angle $\hat{\psi}_{h 1}-\hat{\psi}_{i 1}$ was equal to $5 \pi / 2$, arg $\left\langle\hat{q}_{h 1} / \hat{q}_{i 1}\right\rangle$ would be equal to $\pi / 2$. It follows that there may be several values of $\hat{\psi}_{\mathrm{h} 1}-\hat{\psi}_{\mathrm{il}}$ which could have produced a particular $\hat{q}_{h 1} / \hat{q}_{i 1}$ and this fact makes it impossible for a bearing estimation algorithm to recover the distance $\hat{\epsilon}_{\mathrm{h} 1}-\hat{\epsilon}_{\mathrm{il}}$ 
unambiguously. The following relationship exists between $\hat{q}_{\mathrm{h} 1} / \hat{q}_{\mathrm{i} 1}$ and $\hat{\epsilon}_{\mathrm{h} 1}-\hat{\epsilon}_{\mathrm{i} 1}$.

$$
\hat{\epsilon}_{h 1}-\hat{\epsilon}_{i 1}=\frac{1}{2 \pi} \arg \left\{\frac{\hat{q}_{h 1}}{\hat{q}_{i 1}}\right\}+L
$$

$L$ is an unknown integer which accommodates the ambiguity. Since $\hat{\epsilon}_{h 1}-\hat{\epsilon}_{i 1}$ is the length of the component of $\hat{u}_{h}-\hat{u}_{i}$ in a particular direction, the distance $\left|\hat{\epsilon}_{\mathrm{h} 1}-\hat{\epsilon}_{\mathrm{i} 1}\right|$ cannot be greater than the separation between the two sensors.

$$
\left|\hat{\epsilon}_{h 1}-\hat{\epsilon}_{i 1}\right| \leqslant\left\|\hat{\sim}_{h}-\hat{\sim}_{i}\right\|
$$

Given particular values of $\hat{\sim}_{h 1}, \hat{\sim}_{i 1}, \hat{q}_{h 1}$, and $\hat{q}_{i 1}$, it is possible to determine the feasible values of $L$ by substituting $(3.11)$ into $(3.12)$.

$$
\left|\frac{1}{2 \pi} \arg \left\{\frac{\hat{q}_{h 1}}{\hat{q}_{i 1}}\right\}+L\right| \leqslant\left\|\hat{\sim}_{h}-\hat{\sim}_{i}\right\|
$$

In particular, if the distance between the sensors is less than or equal to half a wavelength, $L$ must be equal to zero and $\hat{\epsilon}_{\mathrm{h} 1}-\hat{\epsilon}_{\mathrm{il}}$ can be recovered unambiguously. Given $\hat{\epsilon}_{\mathrm{h} 1}-\hat{\epsilon}_{\mathrm{i} 1}$, the bearing of the signal can, in principle, be found by solving (3.8) for $z_{1}$. 
The equation can be solved using a graphical

construction. Figure 3.8 identifies a set of right-angled triangles, all of which have the line joining sensors $h$ and $i$ as their hypotenuse. In other words, all of the triangles have one corner at the position of sensor $h$ and another at the position of sensor $i$. Since the triangles are right-angled, the third corner must always lie on the circumference of a circle which has the line joining the two sensors as its diameter. There is an infinite number of such triangles.

Now, $\hat{\epsilon}_{\mathrm{hl}}-\hat{\epsilon}_{\mathrm{il}}$ is the length of one of two orthogonal components of $\hat{u}_{h}-\hat{u}_{i}$. There must exist a right-angled triangle which has the line joining the two sensors as its hypotenuse and the other two sides equal to the two orthogonal components. This triangle must be a member of the set of triangles which has just been discussed.

The relevant triangle is found by drawing an arc of radius $\hat{\epsilon}_{\text {h1 }}-\hat{\epsilon}_{\text {i1 }}$ with its centre at the intended position of sensor $i$ as illustrated in Figure 3.9. The point where the arc intersects the circle defines the third corner of the triangle.

Consider the direction of the line joining sensor $i$ to the third corner of the triangle. The length of the component of $\hat{u}_{h}-\hat{\sim}_{i}$ in that direction is $\hat{\epsilon}_{\mathrm{h} I}-\hat{\epsilon}_{i 1}$ and 


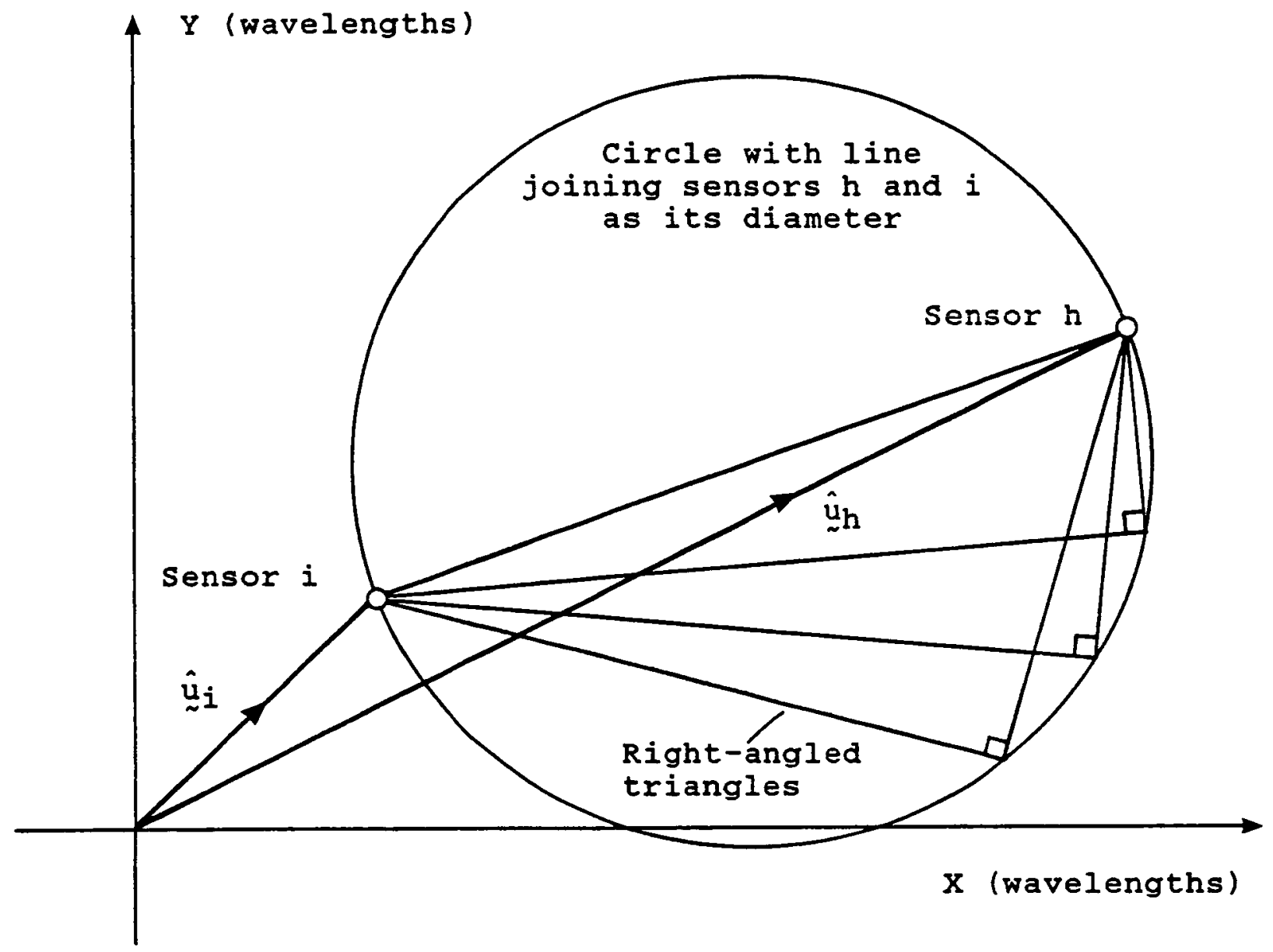

Figure 3.8 set of right-angled triangles 


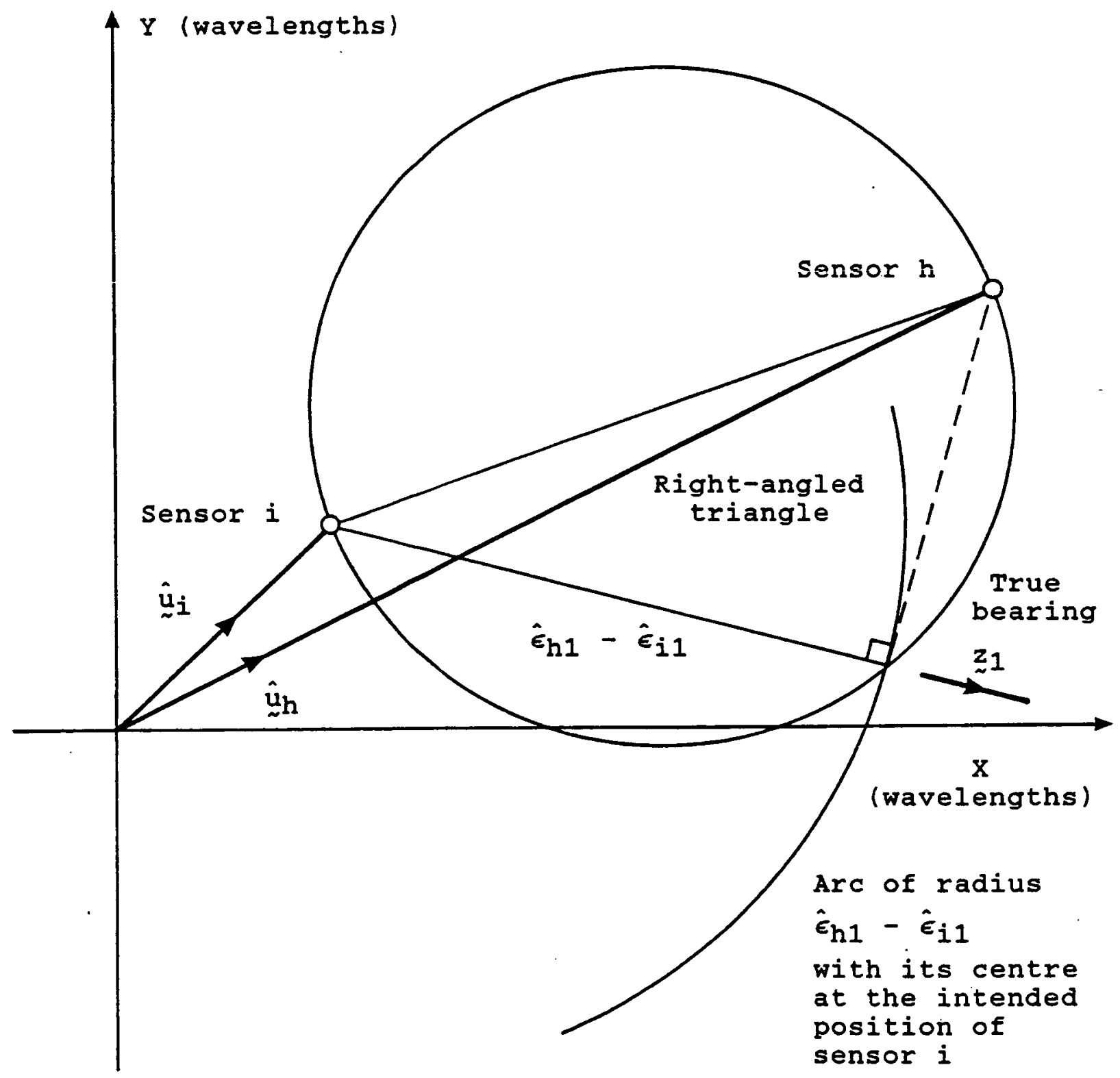

Figure 3.9 Graphical construction to recover bearing from intended positions of sensors $h$ and $i$ 
this is a necessary condition for the line to point in the same direction as the unknown vector $z_{1}$ which defines the bearing of the signal.

Although it is a necessary condition, it is not" a sufficient one since the arc actually intersects the circle in two places as illustrated in Figure 3.10. The construction generates two right-angled triangles and there is no way of telling which one of them defines the bearing of the signal.

To summarize, if sensors $h$ and $i$ are separated by half a wavelength or less, the phasor $\hat{q}_{h 1} / \hat{q}_{i 1}$ reveals the distance $\hat{\epsilon}_{\mathrm{h} 1}-\hat{\epsilon}_{\mathrm{il}}$ unambiguously. A circle which has the line joining sensors $h$ and $i$ as $i t s$ diameter is plotted and an arc of radius $\hat{\epsilon}_{\mathrm{h} 1}-\hat{\epsilon}_{i 1}$ is drawn with its centre at the intended position of sensor $i$. The points of intersection between the circle and the arc suggest two possible bearings for the signal being received and one of these bearings is correct, although there is no way of telling which one.

When sensors $h$ and $i$ are more than half a wavelength apart, $\hat{q}_{h 1} / \hat{q}_{i 1}$ suggests more than one possible length for the component of $\hat{u}_{h}-\hat{u}_{i}$ along the axis of propagation. These lengths are of the form $\hat{\epsilon}_{\mathrm{h} 1}-\hat{\epsilon}_{i 1}-L$ and only one of them has L equal to zero. Each possible length 


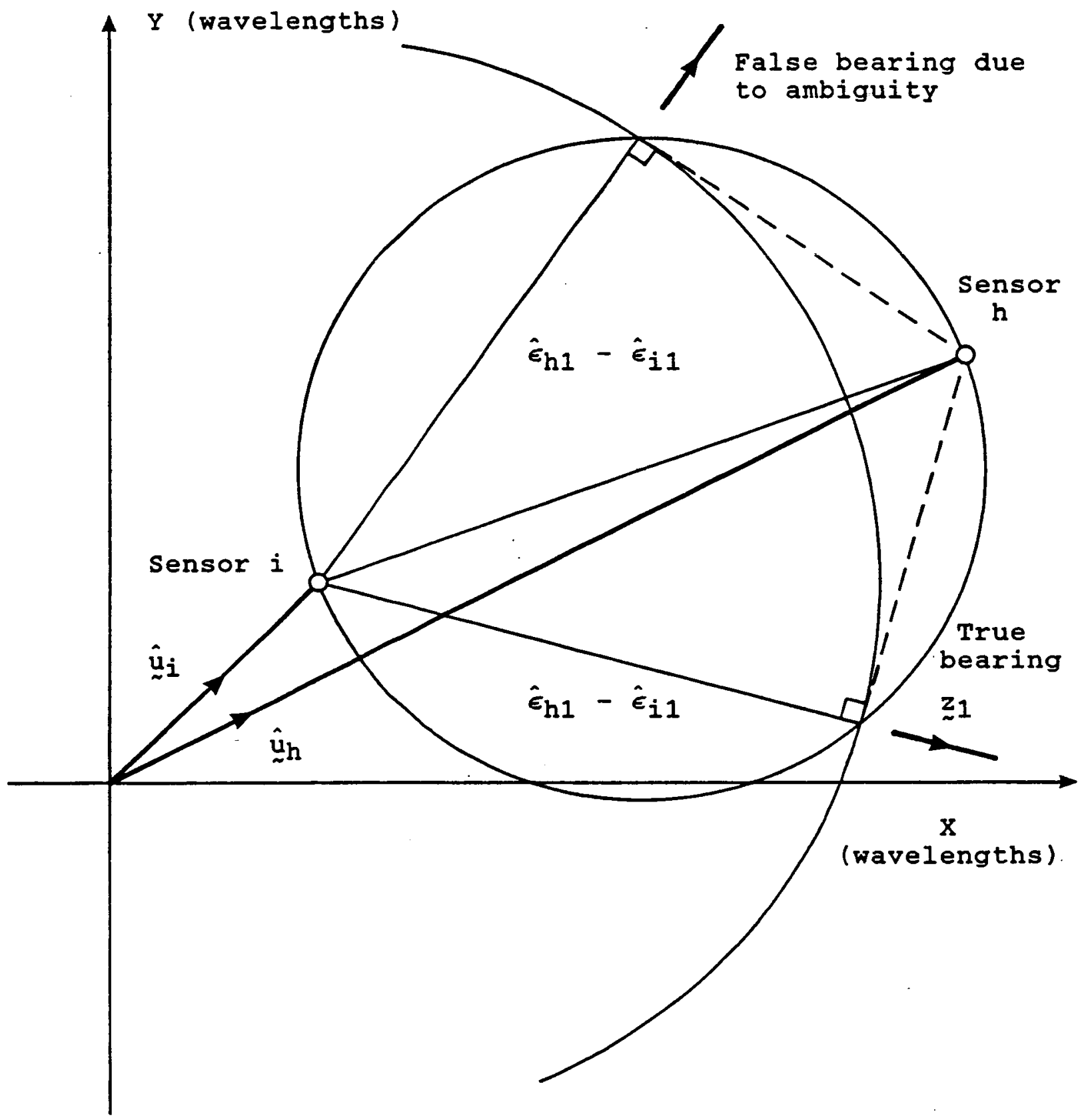

Figure 3.10 Ambiguity in recovery of bearing 
suggests two possible bearings. For example, in Figure 3.11 there is an arc of radius $\hat{\epsilon}_{h 1}-\hat{\epsilon}_{i 1}$ and another of radius $\hat{\epsilon}_{\mathrm{h} 1}-\hat{\epsilon}_{i 1}-1$. Since both of the arcs cut the circle in two places, there are four possible bearings, one of which is correct.

Notice that $\hat{\epsilon}_{\mathrm{h} 1}-\hat{\epsilon}_{\mathrm{i} 1}$ (and $\hat{\epsilon}_{\mathrm{h} 1}-\hat{\epsilon}_{\mathrm{i} 1}-$ L) may be negative. If this happens, the graphical constructions look the same but $\pi$ radians must be added to for subtracted from) all of the bearings produced.

So far, the discussion has shown that the information obtained from a single pair of sensors is not sufficient to identify the bearing of the signal unambiguously. The true bearing does not become apparent until the information from all of the sensor pairs in the array is combined. In an array of $\mathrm{N}$ sensors, there are a total of $N(N-1) / 2$ unique pairs of sensors.

Each sensor pair contributes the true bearing plus one or more other possibilities. The key point is that the spurious bearings are generally different for each pair of sensors. When the information from all of the sensor pairs is combined, the correct bearing is reinforced while the spurious ones are not. 


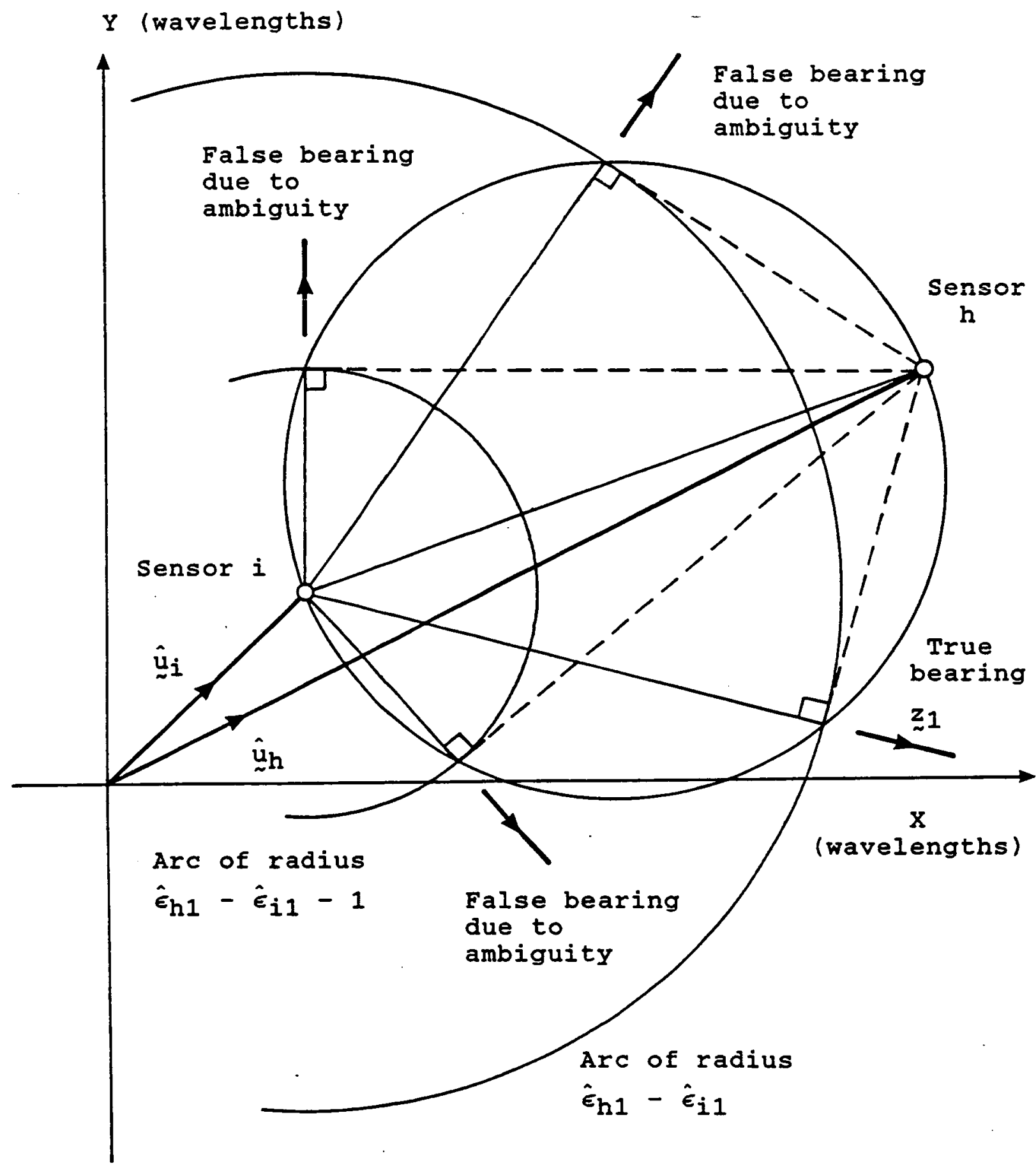

Figure 3.11 Further ambiguities in recovery of bearing 
Figure 3.12 shows the bearings produced by the individual sensor pairs in the array used for the simulations. There is one signal present at a bearing of 18 degrees. Each row shows the bearings produced by one of the 15 unique pairs of sensors within the array and there are either two or four possible bearings in each case. Notice how a solid column is produced at 18 degrees whereas no other bearing is reinforced in this way.

\subsection{The Mean of the Bearing Error}

When sensor positioning errors are present, the process described in the last section can be repeated using the true sensor positions instead of the intended ones. $\hat{u}_{h}$. $\hat{u}_{i}$, and $\hat{\epsilon}_{h 1}-\hat{\epsilon}_{i 1}$ are replaced with $\underline{\sim}_{h}, \underline{\sim}_{i}$, and $\epsilon_{h 1}-\epsilon_{i 1}$ respectively. Recalling the definition of $\epsilon_{n m}$ given in (3.5), the difference $\epsilon_{\mathrm{hl}}-\epsilon_{i 1}$ is as follows.

$$
\begin{aligned}
\epsilon_{\mathrm{h} 1}-\epsilon_{i 1} & =\left(\hat{\epsilon}_{\mathrm{h} 1}-\Delta \epsilon_{\mathrm{h} 1}+\Delta \epsilon_{11}\right)-\left(\hat{\epsilon}_{\mathrm{i} 1}-\Delta \epsilon_{\mathrm{i} 1}+\Delta \epsilon_{11}\right) \\
& =\hat{\epsilon}_{\mathrm{h} 1}-\hat{\epsilon}_{\mathrm{i} 1}-\left(\Delta \epsilon_{\mathrm{h} 1}-\Delta \epsilon_{\mathrm{i} 1}\right)
\end{aligned}
$$

Figure 3.13 summarizes the relationship between $\epsilon_{\mathrm{h} 1}-\epsilon_{\mathrm{il}}$, $\hat{\epsilon}_{\mathrm{h} 1}-\hat{\epsilon}_{\mathrm{il}}$, and the true and intended sensor positions. In reality, only the true sensor positions exist and the 
Sensor pair $(h, i)$

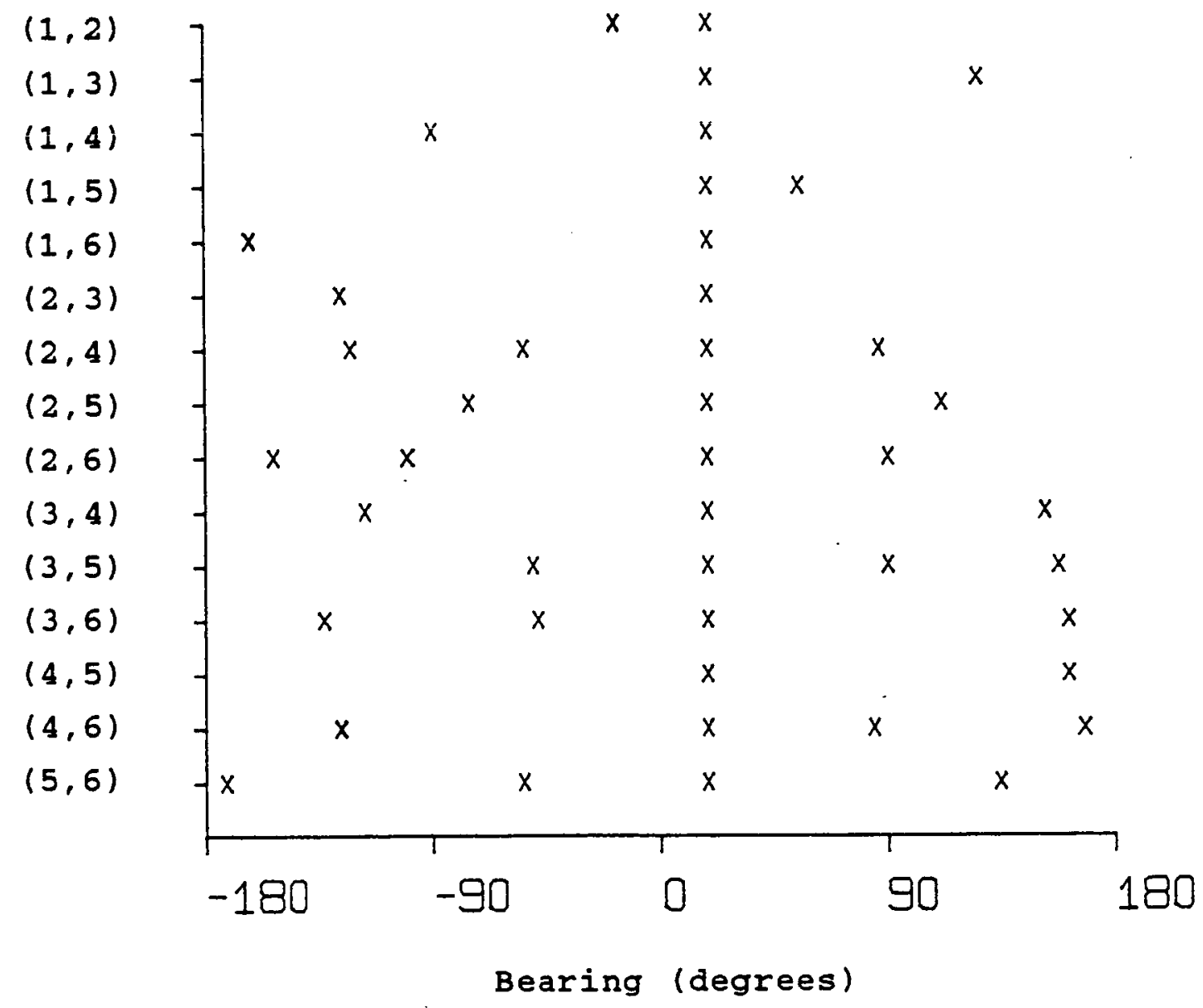

Figure 3.12 Bearings produced by individual pairs of sensors 


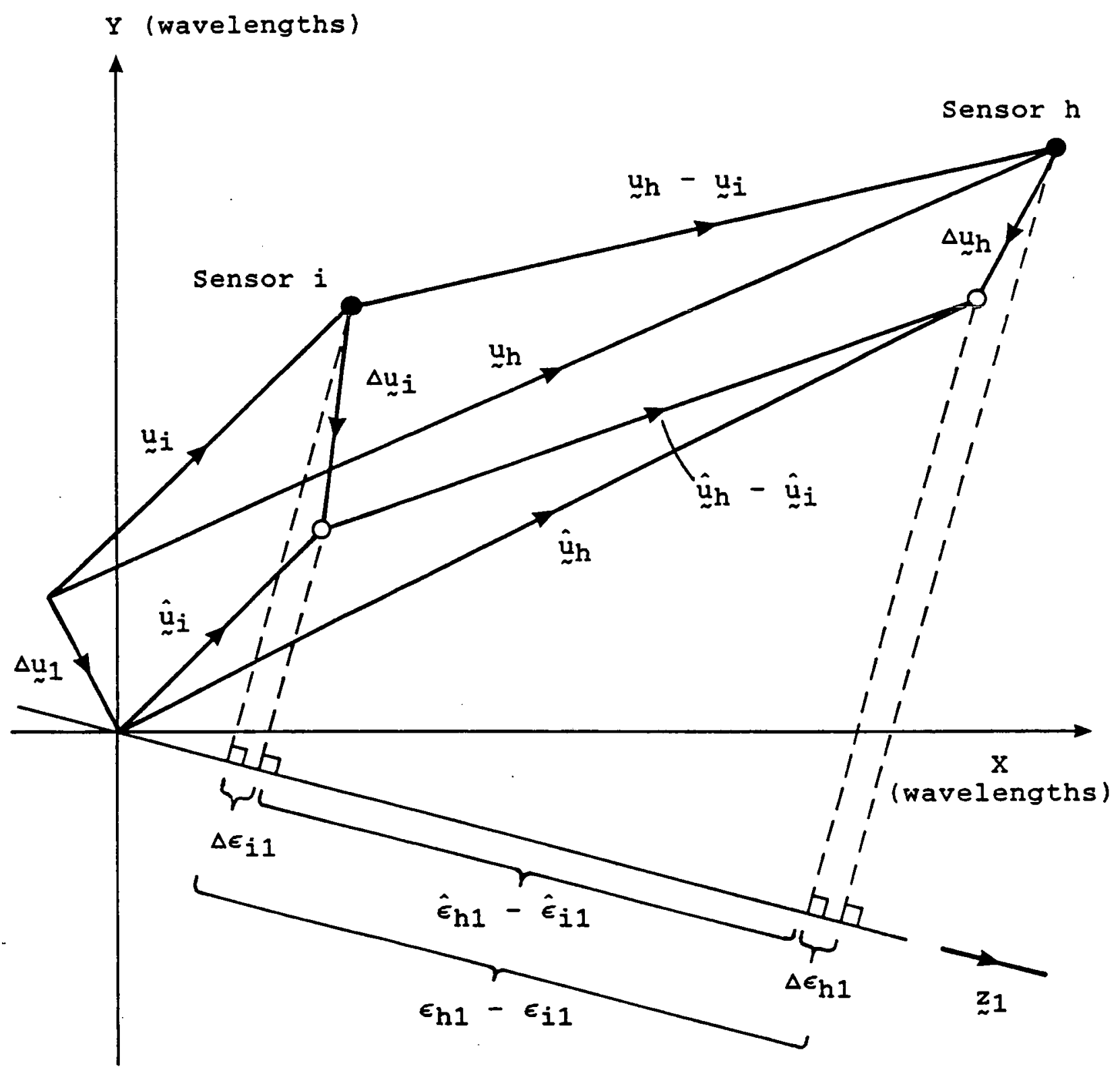

O intended sensor positions

- true sensor positions

Figure 3.13 Distances for true and intended positions of sensors $h$ and $i$ 
sampling process therefore reveals $\epsilon_{\mathrm{hl}}-\epsilon_{\mathrm{il}}$ instead of $\hat{\epsilon}_{h 1}-\hat{\epsilon}_{i 1}$.

If the true sensor positions were known, the bearing of the signal could be sought using the method introduced in the last section. Figure 3.14 shows an arc with a radius of $\hat{\epsilon}_{\mathrm{h} 1}-\hat{\epsilon}_{\mathrm{i} 1}-\left(\Delta \epsilon_{\mathrm{h} 1}-\Delta \epsilon_{\mathrm{i} 1}\right)$, or $\epsilon_{\mathrm{h} 1}-\epsilon_{\mathrm{i} 1}$, with its centre at the true position of sensor $i$ and a circle which has the line joining the true positions of the two sensors as its diameter. The point of intersection between the arc and the circle reveals the bearing of the signal as before.

The diagram also shows the corresponding construction for the intended sensor positions, which is copied directly from Figure 3.9. Notice that both constructions reveal the true bearing of the signal correctly. They both produce spurious bearings as well, although these have been omitted in order to simplify the diagram.

In practice, the sensor positioning errors are unknown and the true sensor positions are assumed to be identical to the intended ones. The result is that $\epsilon_{\mathrm{hl}}-\epsilon_{\mathrm{il}}$ is assumed to be the length of the component of $\hat{u}_{h}-\hat{\sim}_{i}$ in the direction of propagation although it is really the length of the component of ${\underset{\sim}{h}}_{h}-\underline{\sim}_{i}$. 


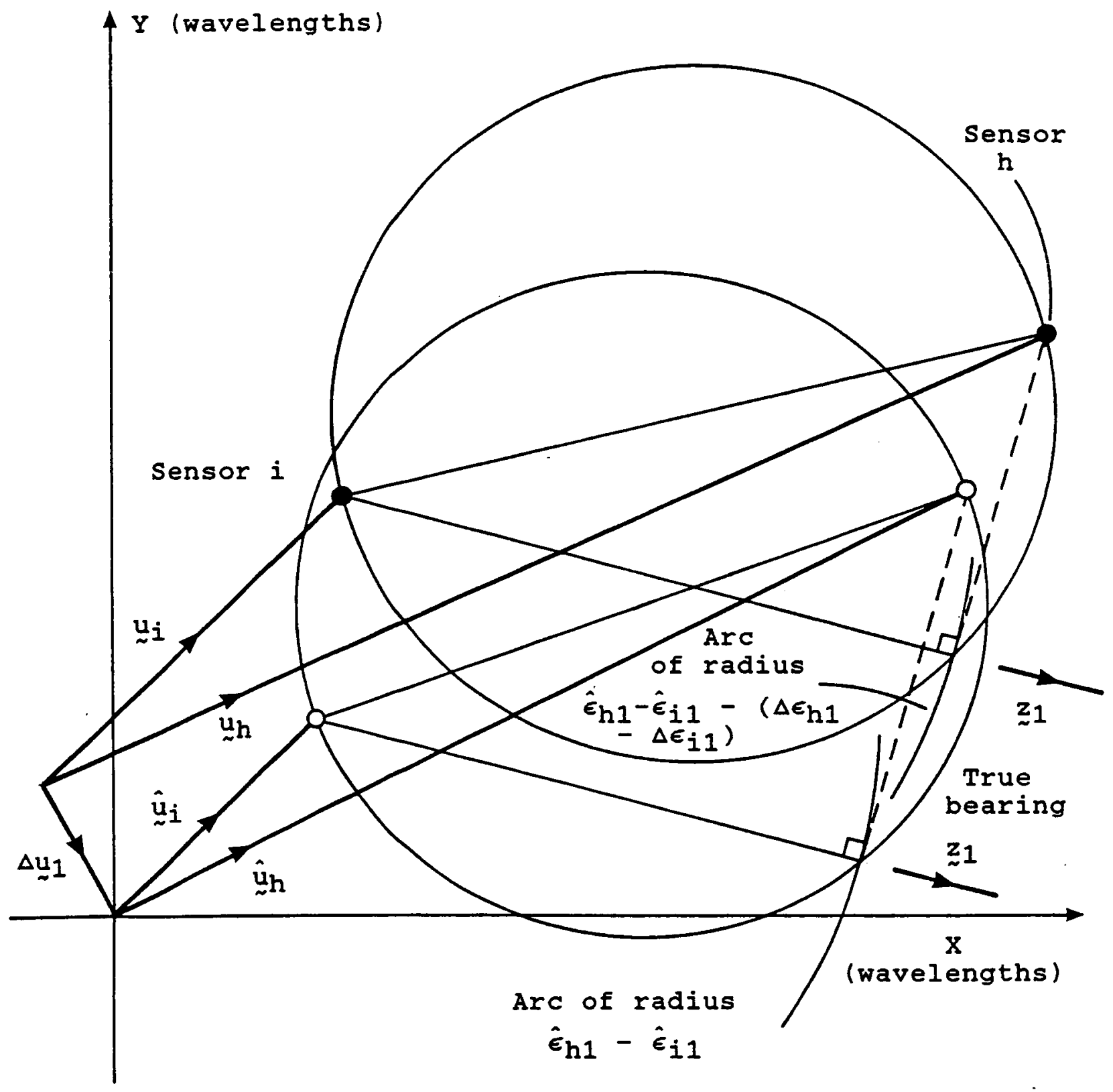

$O$ intended sensor positions

- true sensor positions

Figure 3.14 Graphical construction to recover bearing from true positions of sensors $h$ and $i$ 
Figure 3.15 shows how this misinterpretation of $\epsilon_{\mathrm{h} 1}-\epsilon_{\mathrm{i} 1}$ leads to an error in the bearing of the signal. Having drawn the circle which passes through the intended sensor positions, an arc of radius $\hat{\epsilon}_{\mathrm{h} 1}-\hat{\epsilon}_{i 1}$ is required to identify the signa'l bearing correctly. However, an arc of radius $\hat{\epsilon}_{\mathrm{h} 1}-\hat{\epsilon}_{\mathrm{il}}-\left(\Delta \epsilon_{\mathrm{h} 1}-\Delta \epsilon_{\mathrm{i} 1}\right)$ is used instead and this intersects the circle at the wrong point, causing an error in the bearing of the signal.

The bearing error produced by sensors $h$ and $i$ is denoted by $\left(\Delta \theta_{1}\right)_{h i}$. There are also errors in the spurious bearings produced by the sensor pair, although these do not generally have the same sign and magnitude as $\left(\Delta \theta_{1}\right)_{h i}$.

The sign of $\left(\Delta \theta_{1}\right)_{h i}$ depends on the sign of $\Delta \epsilon_{\mathrm{h} 1}-\Delta \epsilon_{i 1}$, as shown in Figure 3.16. The true signal bearing and the positions of the sensors relative to each other determine whether the sign of $\left(\Delta \theta_{1}\right)_{h i}$ is the same as or opposite to that of $\Delta \epsilon_{\mathrm{h} 1}-\Delta \epsilon_{i 1}$, although it is always true that changing the sign of $\Delta \epsilon_{\mathrm{hl}}-\Delta \epsilon_{\mathrm{il}}$ changes the sign of $\left(\Delta \theta_{1}\right)_{h i}$. For the situation illustrated, the sign of $\left(\Delta \theta_{1}\right)_{h i}$ is opposite to that of $\Delta \epsilon_{\mathrm{h} 1}-\Delta \epsilon_{\mathrm{i} 1}$.

The exaggerated errors used in the diagram reveal that changing the sign of $\Delta \epsilon_{\mathrm{hl}}-\Delta \epsilon_{\mathrm{il}}$ changes the magnitude of $\left(\Delta \theta_{1}\right)_{h i}$ as well as its sign. Comparing Figures $3.16(a)$ and $3.16(\mathrm{~b})$, the size of the bearing error $\left(\Delta \theta_{1}\right)_{h i}$ is 


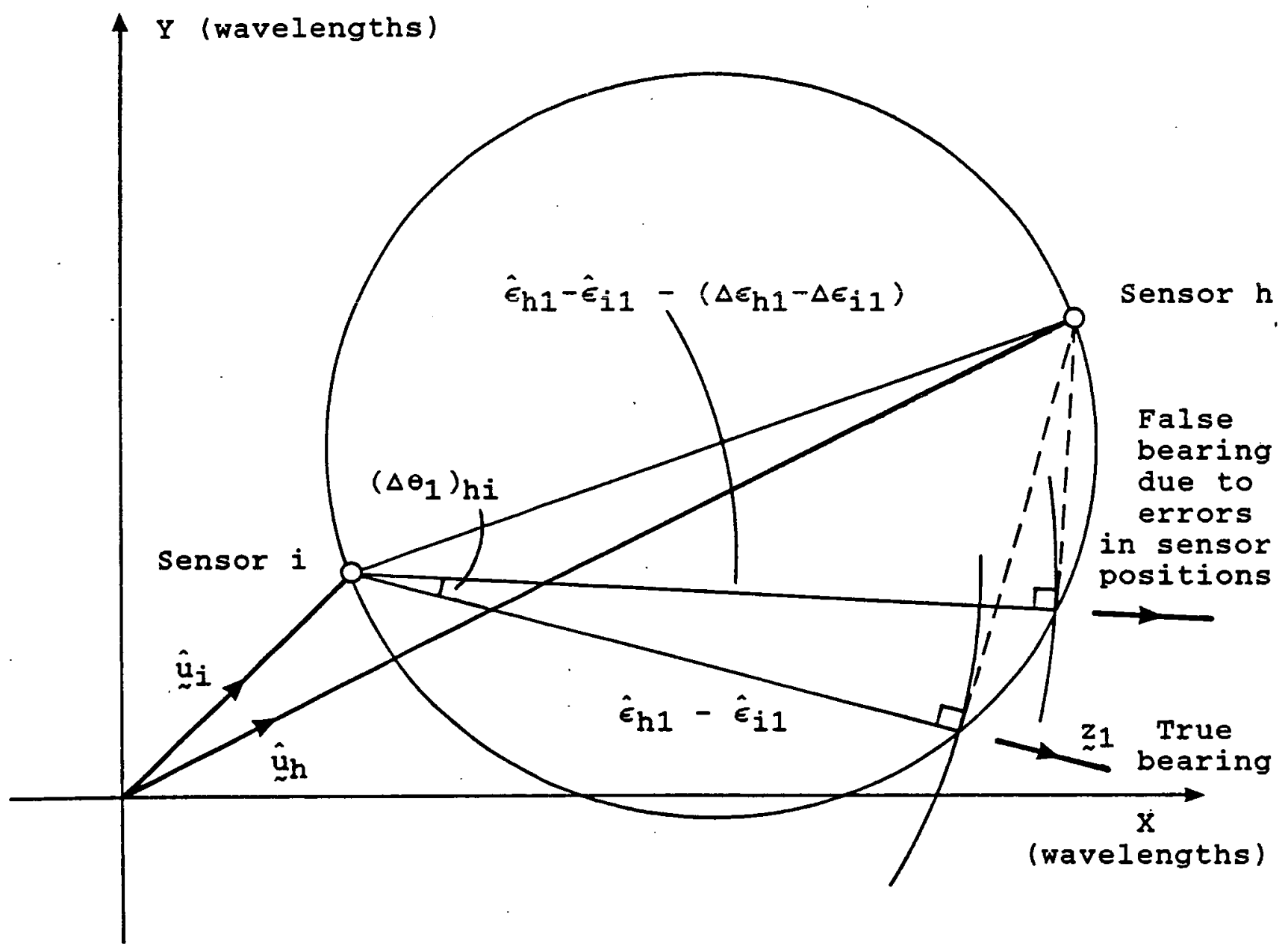

Figure 3.15 Bearing error 


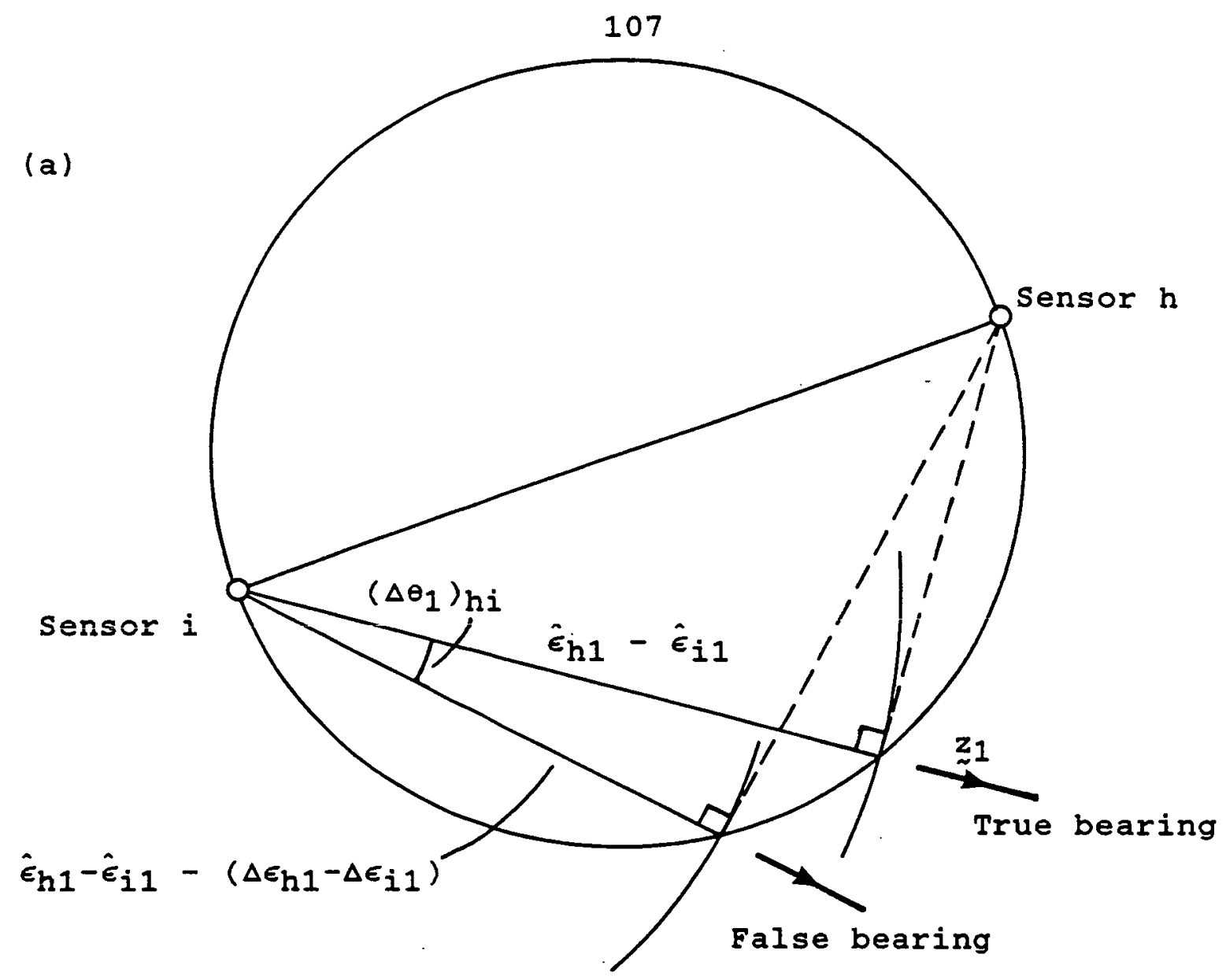

(b)

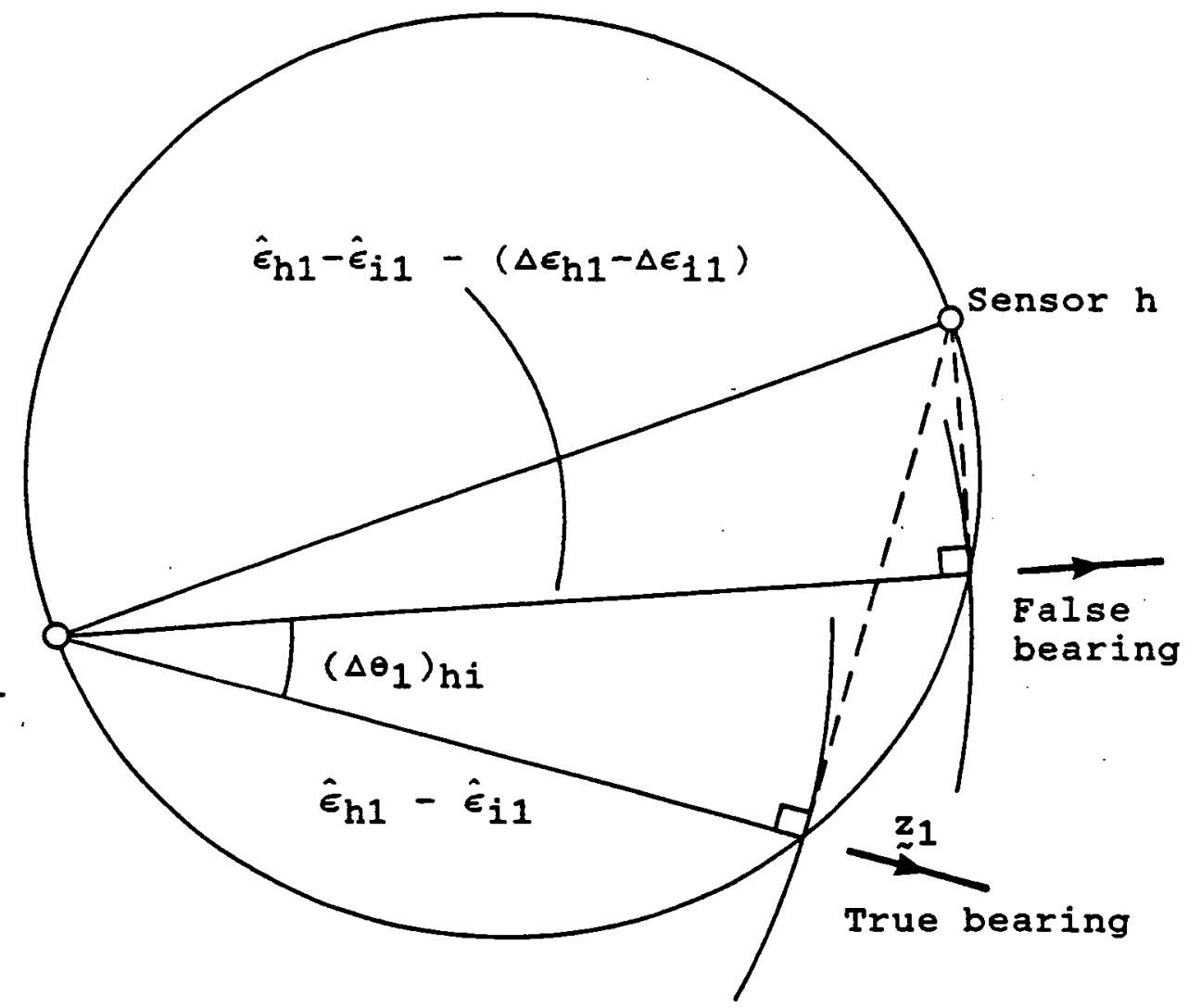

Figure 3.16 Asymmetry in bearing error 
slightly different even though the magnitude of the distance $\Delta \epsilon_{\mathrm{h} 1}-\Delta \epsilon_{\mathrm{il}}$ is the same in both cases. This asymmetry is a natural consequence of the construction used to find $\left(\Delta \theta_{1}\right)_{h i}$.

Notice, however, that the difference between the two magnitudes of $\left(\Delta \theta_{1}\right)_{h i}$ is much smaller than the magnitudes themselves. For small sensor positioning errors, and hence small values of $\left(\Delta \theta_{1}\right)_{h i}$, the difference becomes insignificant and the relationship between $\Delta \epsilon_{\mathrm{h} 1}-\Delta \epsilon_{i 1}$ and $\left(\Delta \theta_{1}\right)_{h i}$ is symmetric. Thus negating $\Delta \epsilon_{\mathrm{hl}}-\Delta \epsilon_{\mathrm{il}}$ negates $\left(\Delta \theta_{1}\right)_{h i}$ without changing its magnitude.

Since $\Delta \epsilon_{\mathrm{hl}}$ and $\Delta \epsilon_{\mathrm{il}}$ both have a normal distribution with a mean of zero and a variance of $\sigma_{p}^{2}$, it follows that the combined error $\Delta \epsilon_{\mathrm{h} 1}-\Delta \epsilon_{i 1}$ has a normal distribution with a mean of zero and a variance of $2 \sigma_{\mathrm{p}}^{2}$. Thus a particular value, say $\rho$, of $\Delta \epsilon_{\mathrm{h} 1}-\Delta \epsilon_{\mathrm{il}}$ has the same probability of occurring as $-p$. Since the values of $\left(\Delta \theta_{1}\right)_{h i}$ corresponding to $e$ and $-e$ have opposite signs but the same magnitude, $\left(\Delta \theta_{1}\right)_{h i}$ must also have a mean of zero.

$$
E\left[\left(\Delta \theta_{1}\right)_{h i}\right]=0
$$

Due to the non-linear relationship between $\Delta \epsilon_{\mathrm{hl}}-\Delta \epsilon_{i 1}$ and $\left(\Delta \theta_{1}\right)_{h i}$, the precise distribution of $\left(\Delta \theta_{1}\right)_{h i}$ is unknown. In the next section, this relationship is explored in 
detail and an approximation for the distribution is developed. However, the fact that the mean of. $\left(\Delta \theta_{1}\right)_{h i}$ is equal to zero is sufficient for the present discussion.

Figure 3.17 shows the effect of small sensor positioning errors on the bearings produced by the individual sensor pairs in the array used for the simulations. There is one signal present at a bearing of 18 degrees as before and the variance of the sensor positions, $\sigma_{p}^{2}$, is equal to 0.001. Comparing this diagram with Figure 3.12 , there is still a well-defined cluster of points in the region of the true bearing. There are, however, small errors in the bearings and each pair of sensors, $h$ and $i$, produces $a$ point at $\theta_{1}+\left(\Delta \theta_{1}\right)_{\text {hi }}$ rather than $\theta_{1}$.

The next step is to relate this diagram to the bearing spectrum, which has a peak at $\theta_{1}+\Delta \theta_{1}$. Two assumptions are required in order to make the problem manageable.

The first assumption is that the cluster over the true bearing in Figure 3.17 contains the same set of $N(N-1) / 2$ points as the column in Figure 3.12, although the bearing of each point has changed slightly. In other words, no point which identifies the true bearing may have such a large bearing error that it is mistaken for a spurious point. Similarly, no spurious point may move so close to the true bearing that it is mistaken for a member of the 
Sensor pair $(h, i)$

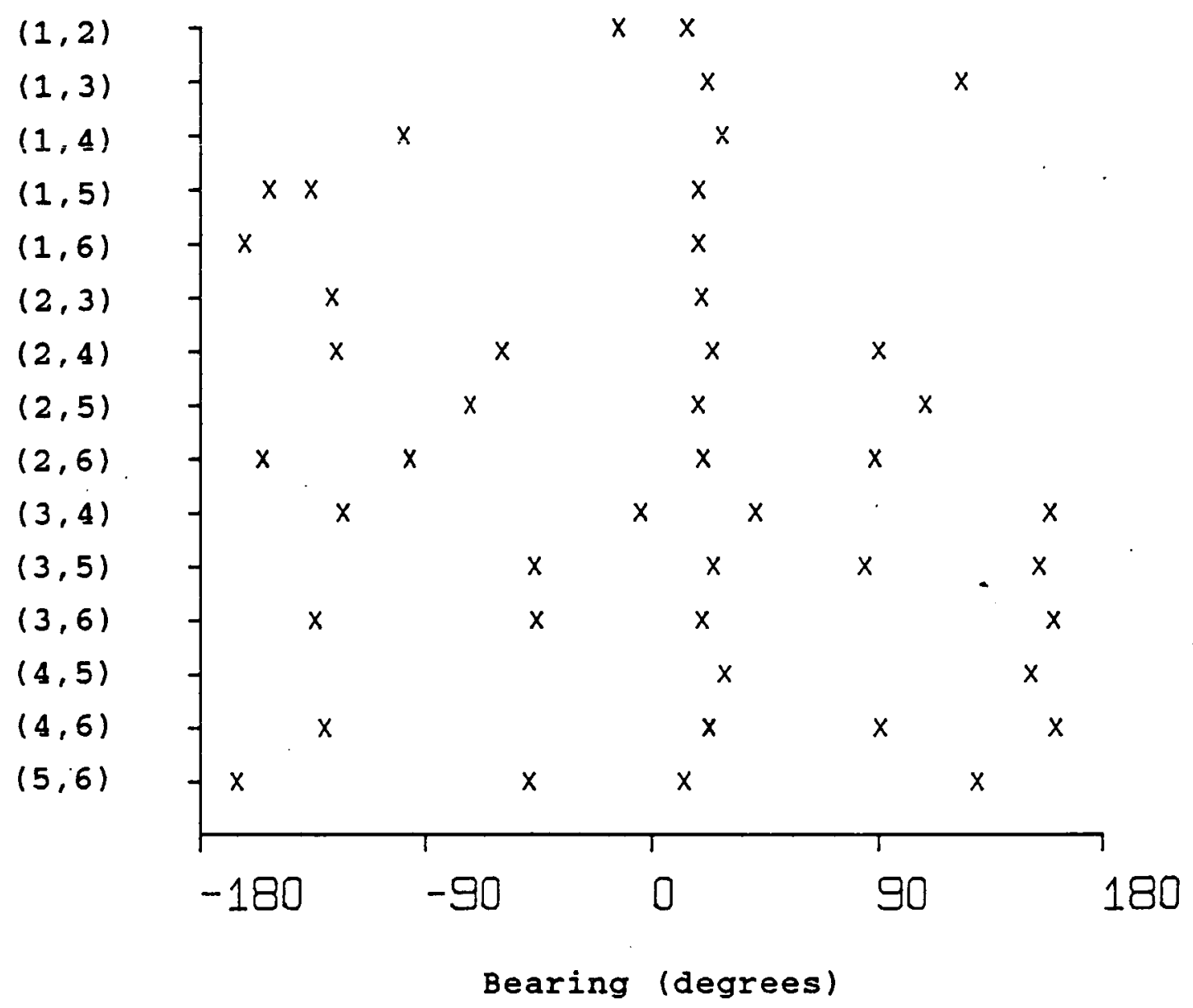

Figure 3.17 Bearing errors produced by individual pairs of sensors 
main cluster.

The second, and most important, assumption is that the bearing of the peak in the spectrum is the average of the bearings indicated by the individual pairs of sensors. The error $\Delta \theta_{1}$ in the bearing of the peak is therefore the average of the $N(N-1) / 2$ errors $\left(\Delta \theta_{1}\right)_{h i}$ produced by the individual sensor pairs.

$$
\Delta \theta_{1}=\frac{2}{N(N-1)} \sum_{h=1}^{N-1} \sum_{i=h+1}^{N}\left(\Delta \theta_{1}\right)_{h i}
$$

From (3.15), the mean of $\left(\Delta \theta_{1}\right)_{h i}$ is zero and it is straightforward to show that the mean of $\Delta \theta_{1}$ is also zero.

$$
\begin{aligned}
E\left[\Delta \theta_{1}\right] & =E\left[\frac{2}{N(N-1)} \sum_{h=1}^{N-1} \sum_{i=h+1}^{N}\left(\Delta \theta_{1}\right)_{h i}\right] \\
& =\frac{2}{N(N-1)} \sum_{h=1}^{N-1} \sum_{i=h+1}^{N} E\left[\left(\Delta \theta_{1}\right)_{h i}\right] \\
& =0
\end{aligned}
$$

In conclusion, bearing estimation algorithms remain unbiased in the presence of sensor positioning errors with 
the assumed statistics. It must be stressed that the array would have to be set up several times to observe this effect. For any particular realization of the sensor positioning errors, there will generally be an error in the bearing of the peak.

These deductions are confirmed by the results from the computer simulation experiment described earlier in the chapter. When the bearing error $\Delta \theta_{1}$ was averaged over several sets of true sensor positions, the resulting estimate of $E\left[\Delta \theta_{1}\right]$ was close to zero. As the number of true arrays was increased, the average of $\Delta \theta_{1}$ moved even closer to zero, suggesting that the true value of $E\left[\Delta \theta_{1}\right]$ was indeed zero as predicted.

Figure 3.18 shows the results obtained using 1000 sets of true sensor positions over a range of values of $\sigma_{p}^{2}$. As anticipated, the results produced by the three bearing estimation techniques were identical.

\subsection{The Variance of the Bearing Error}

This section develops an expression for the variance of the bearing error $\Delta \theta_{1}$ in terms of the variance of the sensor positions $\sigma_{p}^{2}$. The expression is found to depend 
(a) $E\left[\Delta \theta_{1}\right]$ (degrees)

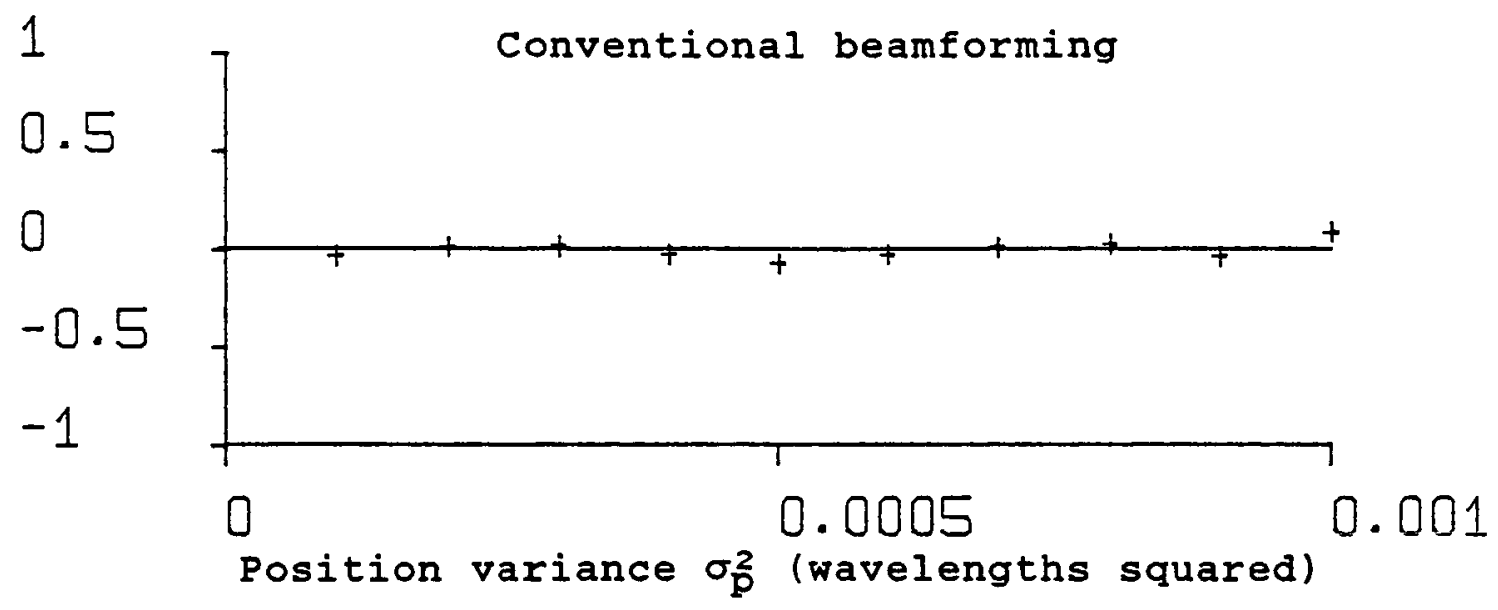

(b) $E\left[\Delta \theta_{1}\right]$ (degrees)

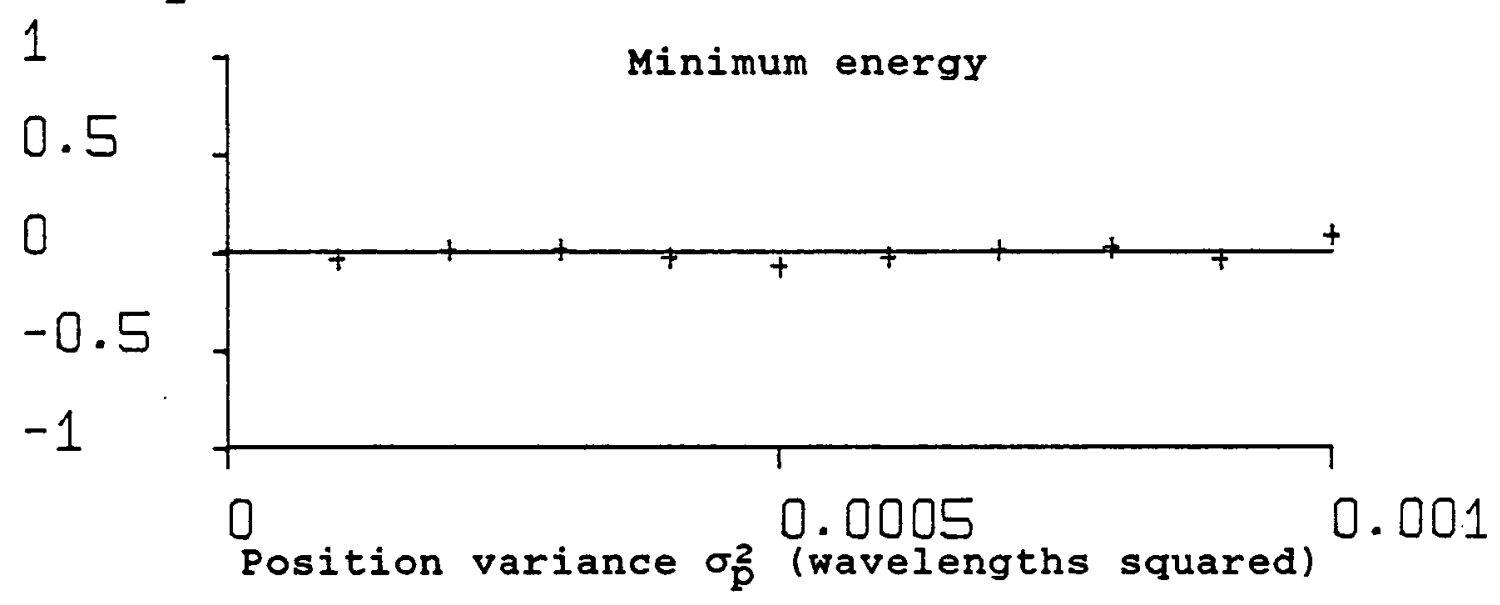

(c) $E\left[\Delta \theta_{1}\right]$ (degrees)

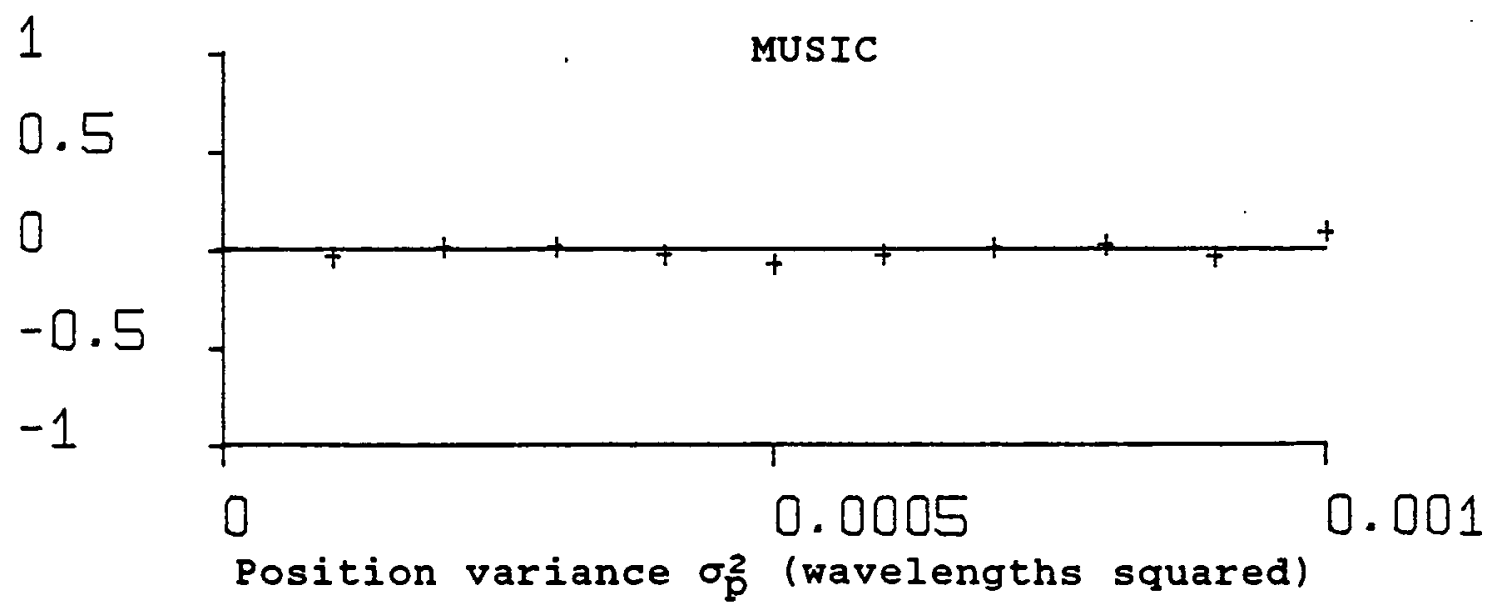

Figure 3.18 Results for mean of bearing error 
on the intended sensor positions $\hat{u}_{n}$ and the bearing of the signal $\theta_{1}$ as well as $\sigma_{p}^{2}$.

Although a geometric view of the relationship between the distance $\Delta \epsilon_{\mathrm{h} 1}-\Delta \epsilon_{\mathrm{il}}$ and the angle $\left(\Delta \theta_{1}\right)_{\mathrm{hi}}$ was sufficient in the last section to discover that the mean of $\left(\Delta \theta_{1}\right)_{h i}$ was zero, a mathematical expression for the relationship is required to allow the variance of $\left(\Delta \theta_{1}\right)_{h i}$ to be calculated.

The first step is to derive a precise expression for $\left(\Delta \theta_{1}\right)_{h i}$ in terms of $\hat{\epsilon}_{h 1}-\hat{\epsilon}_{i 1}, \Delta \epsilon_{h 1}-\Delta \epsilon_{i 1}$, and the separation $\left\|\hat{u}_{h}-\hat{u}_{i}\right\|$ between the two sensors. This expression is found to be intractable due to the presence of non-linear functions of $\Delta \epsilon_{\mathrm{h} 1}-\Delta \epsilon_{i 1}$, which is itself a random variable and the next step is therefore to find an approximation with a form which allows the analysis to proceed. The precise expression is used to check the approximation. Finally, the variance of $\Delta \epsilon_{\mathrm{hl}}-\Delta \epsilon_{i 1}$ is related to the variance of $\left(\Delta \theta_{1}\right)_{h i}$, and hence to that of $\Delta \theta_{1}$

The precise expression for $\left(\Delta \theta_{1}\right)_{h i}$ is based on the construction shown in Figure 3.19 , which is identical to the diagrams used in the last section except that some further angles and distances have been identified. In particular, the angle between the true bearing of the 


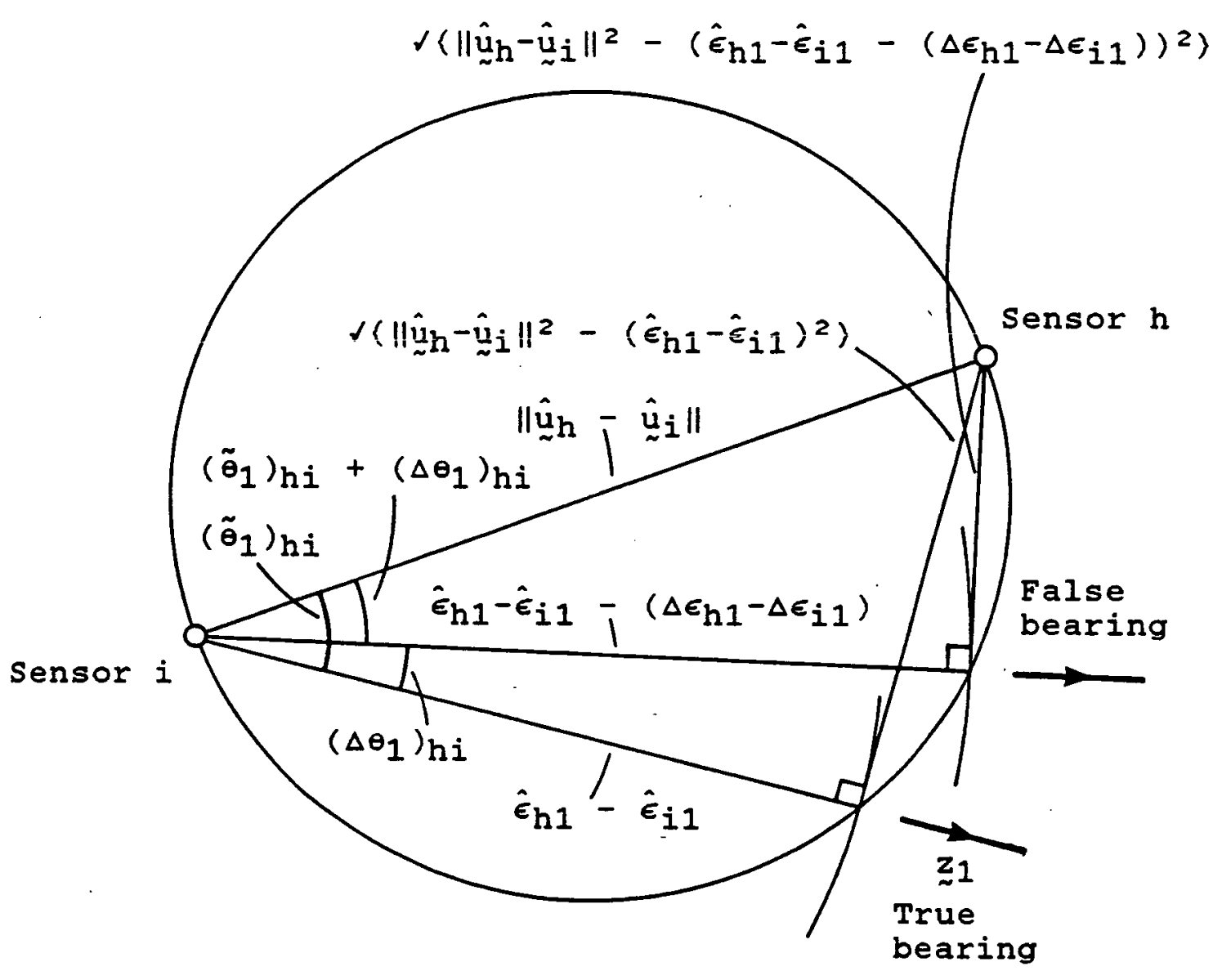

Figure 3.19 Graphical construction for precise bearing error 
signal and the line joining sensors $h$ and $i$ is denoted by $\left(\tilde{\theta}_{1}\right)_{\mathrm{hi}}$.

In the illustration, $\Delta \epsilon_{\mathrm{h} 1}-\Delta \epsilon_{\mathrm{il}}$ is negative. As explained in the last section, the relationship between $\Delta \epsilon_{\mathrm{h} 1}-\Delta \epsilon_{\mathrm{i} 1}$ and $\left(\Delta \theta_{1}\right)_{\mathrm{hi}}$ is assumed to be symmetric for small sensor positioning errors and it follows that cases where $\Delta \epsilon_{h 1}-\Delta \epsilon_{i 1}$ is positive are also accommodated. For the sensor positions and the signal bearing shown, the sign of $\left(\Delta \theta_{1}\right)_{h i}$ is opposite to that of $\Delta \epsilon_{\mathrm{hl}}-\Delta \epsilon_{\mathrm{il}}$. It is not, however, necessary to generalize this relationship since $\left(\Delta \theta_{1}\right)_{h i}$ is squared when calculating the variance, thus losing the sign.

The diagram shows two right-angled triangles, both of which have a hypotenuse of length $\left\|\hat{\sim}_{h}-\hat{\sim}_{i}\right\|$. The triangle which generates the true bearing has one side with a length of $\hat{\epsilon}_{\mathrm{h} 1}-\hat{\epsilon}_{\mathrm{il}}$ and it follows from. Pythagoras' theorem that the remaining side of this triangle has a length of $\checkmark\left(\left\|\hat{\sim}_{\mathrm{h}}-\hat{\sim}_{i}\right\|^{2}-\left(\hat{\epsilon}_{\mathrm{h} 1}-\hat{\epsilon}_{i 1}\right)^{2}\right\rangle$. Similarly, the lengths of the shorter sides of the other triangle are found to be $\hat{\epsilon}_{\mathrm{h} 1}-\hat{\epsilon}_{i 1}-\left(\Delta \epsilon_{\mathrm{h} 1}-\Delta \epsilon_{i 1}\right)$ and $v\left(\left\|\hat{u}_{\mathrm{h}}-\hat{u}_{i}\right\|^{2}-\left(\hat{\epsilon}_{\mathrm{h} 1}-\hat{\epsilon}_{i 1}-\left(\Delta \epsilon_{\mathrm{h} 1}-\Delta \epsilon_{i 1}\right)\right)^{2}\right)$.

Having obtained the lengths of the sides of the triangles, it is possible to write expressions for the sines and cosines of the angles $\left(\tilde{\theta}_{1}\right)_{h i}$ and $\left(\tilde{\theta}_{1}\right)_{h i}+\left(\Delta \theta_{1}\right)_{h i}$. 


$$
\begin{aligned}
& \sin \left[\left(\tilde{\theta}_{1}\right)_{h i}\right]=\frac{\gamma\left(\left\|\hat{u}_{h}-\hat{\sim}_{i}\right\|^{2}-\left(\hat{\epsilon}_{h 1}-\hat{\epsilon}_{i 1}\right)^{2}\right\rangle}{\left\|\hat{\sim}_{h}-\hat{\underline{u}}_{i}\right\|} \\
& \cos \left[\left(\tilde{\theta}_{1}\right)_{h i}\right]=\frac{\hat{\epsilon}_{h 1}-\hat{\epsilon}_{i 1}}{\left\|\hat{u}_{h}-\hat{\sim}_{i}\right\|}
\end{aligned}
$$

$$
\begin{aligned}
& \sin \left[\left(\tilde{\theta}_{1}\right)_{h i}+\left(\Delta \theta_{1}\right)_{h i}\right] \\
& =\frac{v\left(\left\|\hat{\sim}_{h}-\hat{u}_{i}\right\|^{2}-\left(\hat{\epsilon}_{h 1}-\hat{\varepsilon}_{i 1}-\left(\Delta \epsilon_{h 1}-\Delta \epsilon_{i 1}\right)\right)^{2}\right)}{\left\|\underline{\sim}_{h}-\hat{\sim}_{i}\right\|}
\end{aligned}
$$

$$
\cos \left[\left(\tilde{\theta}_{1}\right)_{h i}+\left(\Delta \theta_{1}\right)_{h i}\right]=\frac{\hat{\epsilon}_{h 1}-\hat{\epsilon}_{i 1}-\left(\Delta \epsilon_{h 1}-\Delta \epsilon_{i 1}\right)}{\left\|\hat{\sim}_{h}-\hat{\sim}_{i}\right\|}
$$

The sine and cosine of $\left(\tilde{\theta}_{1}\right)_{h i}+\left(\Delta \theta_{1}\right)_{h i}$ can be related to the sines and cosines of $\left(\tilde{\theta}_{1}\right)_{h i}$ and $\left(\Delta \theta_{1}\right)_{h i}$ using double-angle formulae.

$$
\begin{aligned}
\sin \left[\left(\tilde{\theta}_{1}\right)_{h i}+\right. & \left.\left(\Delta \theta_{1}\right)_{h i}\right]=\sin \left[\left(\tilde{\theta}_{1}\right)_{h i}\right] \cos \left[\left(\Delta \theta_{1}\right)_{h i}\right] \\
& +\cos \left[\left(\tilde{\theta}_{1}\right)_{h i}\right] \sin \left[\left(\Delta \theta_{1}\right)_{h i}\right] \\
\cos \left[\left(\tilde{\theta}_{1}\right)_{h i}+\right. & \left.\left(\Delta \theta_{1}\right)_{h i}\right]=\cos \left[\left(\tilde{\theta}_{1}\right)_{h i}\right] \cos \left[\left(\Delta \theta_{1}\right)_{h i}\right] \\
& -\sin \left[\left(\tilde{\theta}_{1}\right)_{h i}\right] \sin \left[\left(\Delta \theta_{1}\right)_{h i}\right]
\end{aligned}
$$

Substitute $(3.18),(3.19),(3.20)$, and (3.21) into (3.22) and $(3.23)$. 


$$
\begin{aligned}
& \frac{v\left\langle\left\|\hat{u}_{h}-\hat{u}_{i}\right\|^{2}-\left(\hat{\epsilon}_{\mathrm{h} 1}-\hat{\epsilon}_{i 1}-\left(\Delta \epsilon_{\mathrm{h} 1}-\Delta \epsilon_{i 1}\right)\right)^{2}\right\rangle}{\left\|\hat{\sim}_{\mathrm{h}}-\hat{\sim}_{i}\right\|} \\
& =\frac{v\left(\left\|\hat{\sim}_{h}-\hat{\sim}_{i}\right\|^{2}-\left(\hat{\epsilon}_{h 1}-\hat{\epsilon}_{i 1}\right)^{2}\right\rangle}{\left\|\hat{\sim}_{h}-\hat{\sim}_{i}\right\|} \cos \left[\left(\Delta \theta_{1}\right)_{h i}\right] \\
& +\frac{\hat{\epsilon}_{\mathrm{h} 1}-\hat{\epsilon}_{i 1}}{\left\|\hat{u}_{\mathrm{h}}-\hat{\underline{u}}_{i}\right\|} \sin \left[\left(\Delta \theta_{1}\right)_{\mathrm{hi}}\right] \\
& \frac{\hat{\epsilon}_{\mathrm{h} 1}-\hat{\epsilon}_{i 1}-\left(\Delta \epsilon_{\mathrm{h} 1}-\Delta \epsilon_{i 1}\right)}{\left\|\hat{u}_{\mathrm{h}}-\hat{\sim}_{i}\right\|} \\
& =\frac{\hat{\epsilon}_{\mathrm{h} 1}-\hat{\epsilon}_{i 1}}{\left\|\hat{u}_{\mathrm{h}}-\hat{\underline{u}}_{i}\right\|} \cos \left[\left(\Delta \theta_{1}\right)_{\mathrm{hi}}\right] \\
& -\frac{\Delta\left\langle\left\|\hat{\sim}_{h}-\hat{\sim}_{i}\right\|^{2}-\left(\hat{\epsilon}_{h 1}-\hat{\epsilon}_{i 1}\right)^{2}\right\}}{\left\|\hat{u}_{h}-\hat{u}_{i}\right\|} \sin \left[\left(\Delta \theta_{1}\right)_{h i}\right]
\end{aligned}
$$

Now multiply (3.24) by $\left\|{\underset{\sim}{\mathrm{u}}}_{\mathrm{h}}-{\underset{\sim}{\mathrm{u}}}_{i}\right\|\left(\hat{\epsilon}_{\mathrm{h} 1}-\hat{\epsilon}_{\mathrm{i} 1}\right)$ and (3.25) by $\left\|\hat{u}_{h}-\hat{u}_{i}\right\| r\left(\left\|\hat{u}_{h}-\hat{u}_{i}\right\|^{2}-\left(\hat{\epsilon}_{h 1}-\hat{\epsilon}_{i 1}\right)^{2}\right)$.

$$
\begin{aligned}
& \left(\hat{\epsilon}_{\mathrm{h} 1}-\hat{\epsilon}_{\mathrm{i} 1}\right) \vee\left(\left\|\hat{\sim}_{\mathrm{h}}-\hat{\sim}_{i}\right\|^{2}-\left(\hat{\epsilon}_{\mathrm{h} 1}-\hat{\epsilon}_{i 1}-\left(\Delta \epsilon_{\mathrm{h} 1}-\Delta \epsilon_{i 1}\right)\right)^{2}\right\} \\
& =\left(\hat{\epsilon}_{\mathrm{h} 1}-\hat{\epsilon}_{i 1}\right) \checkmark\left(\left\|\hat{\sim}_{\mathrm{h}}-\hat{\sim}_{i}\right\|^{2}-\left(\hat{\epsilon}_{\mathrm{h} 1}-\hat{\epsilon}_{i 1}\right)^{2}\right) \cos \left[\left(\Delta \theta_{1}\right)_{\mathrm{h} i}\right] \\
& +\left(\hat{\epsilon}_{\mathrm{h} 1}-\hat{\epsilon}_{\mathrm{i} 1}\right)^{2} \sin \left[\left(\Delta \theta_{1}\right)_{\mathrm{h} i}\right]
\end{aligned}
$$




$$
\begin{aligned}
& \left(\hat{\epsilon}_{\mathrm{h} 1}-\hat{\epsilon}_{i 1}-\left(\Delta \epsilon_{\mathrm{h} 1}-\Delta \epsilon_{i 1}\right)\right) \checkmark\left(\left\|_{\sim} \hat{\sim}_{\mathrm{h}}-{\underset{\sim}{\mathrm{u}}}_{i}\right\|^{2}-\left(\hat{\epsilon}_{\mathrm{h} 1}-\hat{\epsilon}_{i 1}\right)^{2}\right) \\
& =\left(\hat{\epsilon}_{\mathrm{h} 1}-\hat{\epsilon}_{i 1}\right) \vee\left(\left\|\hat{\sim}_{\mathrm{h}}-\hat{\sim}_{i}\right\|^{2}-\left(\hat{\epsilon}_{\mathrm{h} 1}-\hat{\epsilon}_{i 1}\right)^{2}\right) \cos \left[\left(\Delta \theta_{1}\right)_{h i}\right] \\
& -\left(\left\|\hat{\sim}_{\mathrm{h}}-\hat{\sim}_{i}\right\|^{2}-\left(\hat{\epsilon}_{\mathrm{h} 1}-\hat{\epsilon}_{i 1}\right)^{2}\right) \sin \left[\left(\Delta \theta_{1}\right)_{h i}\right](3.27)
\end{aligned}
$$

Subtract (3.27) from (3.26), thus eliminating $\cos \left[\left(\Delta \theta_{1}\right)_{h i}\right]$.

$$
\begin{gathered}
\left(\hat{\epsilon}_{\mathrm{h} 1}-\hat{\epsilon}_{i 1}\right) \checkmark\left(\left\|\hat{\sim}_{\mathrm{h}}-\hat{\sim}_{i}\right\|^{2}-\left(\hat{\epsilon}_{\mathrm{h} 1}-\hat{\epsilon}_{i 1}-\left(\Delta \epsilon_{\mathrm{h} 1}-\Delta \epsilon_{\mathrm{i} 1}\right)\right)^{2}\right) \\
-\left(\hat{\epsilon}_{\mathrm{h} 1}-\hat{\epsilon}_{i 1}-\left(\Delta \epsilon_{\mathrm{h} 1}-\Delta \epsilon_{i 1}\right)\right) \vee\left(\left\|{\underset{\sim}{\mathrm{u}}}_{\mathrm{h}}-\hat{\sim}_{i}\right\|^{2}-\left(\hat{\epsilon}_{\mathrm{h} 1}-\hat{\epsilon}_{i 1}\right)^{2}\right) \\
=\left\|\hat{\sim}_{\mathrm{h}}-\hat{\sim}_{i}\right\|^{2} \sin \left[\left(\Delta \theta_{1}\right)_{\mathrm{h} i}\right]
\end{gathered}
$$

Now rearrange $(3.28)$ to find $\sin \left[\left(\Delta \theta_{1}\right)_{h i}\right]$.

$$
\begin{aligned}
\sin \left[\left(\Delta \theta_{1}\right)_{h i}\right] & =\frac{1}{\left\|\hat{\sim}_{h}-\hat{\sim}_{i}\right\|^{2}}\left[( \hat { \epsilon } _ { \mathrm { h } 1 } - \hat { \epsilon } _ { i 1 } ) \downarrow \left\{\left\|\hat{\sim}_{\mathrm{h}}-\hat{\sim}_{i}\right\|^{2}\right.\right. \\
& \left.-\left(\hat{\epsilon}_{\mathrm{h} 1}-\hat{\epsilon}_{i 1}-\left(\Delta \epsilon_{\mathrm{h} 1}-\Delta \epsilon_{i 1}\right)\right)^{2}\right\} \\
& -\left(\hat{\epsilon}_{\mathrm{h} 1}-\hat{\epsilon}_{i 1}-\left(\Delta \epsilon_{\mathrm{h} 1}-\Delta \epsilon_{i 1}\right)\right) \vee\left\{\left\|\hat{\sim}_{\mathrm{h}}-\hat{\sim}_{i}\right\|^{2}\right. \\
& \left.\left.-\left(\hat{\epsilon}_{\mathrm{h} 1}-\hat{\epsilon}_{\mathrm{i} 1}\right)^{2}\right\}\right]
\end{aligned}
$$


For particular values of $\Delta \epsilon_{\mathrm{h} 1}$ and $\Delta \epsilon_{i 1}, \sin \left[\left(\Delta \theta_{1}\right)_{\mathrm{hi}}\right]$ can be calculated precisely using (3.29). Results of this type, however, are not sufficiently general in the context of the statistical analysis being attempted. $\Delta \epsilon_{\mathrm{h} 1}$ and $\Delta \epsilon_{i 1}$ are random variables with a known distribution and it should, in principle, be possible to express the variance of $\sin \left[\left(\Delta \theta_{1}\right)_{h i}\right]$, denoted by $v\left[\sin \left[\left(\Delta \theta_{1}\right)_{h i}\right]\right]$, in terms of $\left\|\hat{u}_{\sim_{1}}-\hat{u}_{i}\right\|, \hat{\epsilon}_{h 1}-\hat{\epsilon}_{i 1}$, and the variance $\sigma_{\mathrm{p}}^{2}$ of the sensor positions. Since $\left(\Delta \theta_{1}\right)_{h i}$ is a small angle, it is approximately equal to its own sine and the variance of the sine is a good approximation to the variance of the angle.

$$
\begin{aligned}
& \left(\Delta \theta_{1}\right)_{h i} \simeq \sin \left[\left(\Delta \theta_{1}\right)_{h i}\right] \\
& v\left[\left(\Delta \theta_{1}\right)_{h i}\right]=v\left[\sin \left[\left(\Delta \theta_{1}\right)_{h i}\right]\right]
\end{aligned}
$$

However, the form of the expression for $\sin \left[\left(\Delta \theta_{1}\right)_{\mathrm{hi}}\right]$ given in (3.29) makes it difficult, if not impossible, to obtain $v\left[\sin \left[\left(\Delta \theta_{1}\right)_{h i}\right]\right]$. The problem is caused by the presence of a non-linear function of $\Delta \epsilon_{\mathrm{h} 1}-\Delta \epsilon_{i 1}$ (i.e. the square within the square root), which distorts the distribution of $\Delta \epsilon_{\mathrm{h} 1}-\Delta \epsilon_{\mathrm{il}}$, creating a complicated relationship between the variance of $\Delta \epsilon_{h 1}-\Delta \epsilon_{i l}$ and that of the whole expression.

Although the precise expression for $\left(\Delta \theta_{1}\right)_{h i}$, or rather $\sin \left[\left(\Delta \theta_{1}\right)_{h i}\right]$, is intractable, it is possible to form an 
approximation which leads to a much simpler expression. The precise expression in (3.29) is still useful, however, as it can be used to check the approximation numerically.

Figure 3.20 highlights the shape formed by the two arcs and the two straight lines enclosing the angle $\left(\Delta \theta_{1}\right)_{\mathrm{hi}}$. This shape is a segment of an annulus, although when the distance $\Delta \epsilon_{\mathrm{hl}}-\Delta \varepsilon_{\mathrm{il}}$ and the angle $\left(\Delta \theta_{1}\right)_{\mathrm{hi}}$ are small, the shape closely resembles a rectangle. Figure $3.21(a)$ shows a construction in which tangents to the arcs have been used in place of the arcs themselves, resulting in a rectangle which has a tangent to the circle as its diagonal.

An isosceles triangle is formed by drawing a radius of the circle which is perpendicular to the diagonal of the rectangle. By inspection, the angles at the corners of this triangle are $\left(\tilde{\theta}_{1}\right)_{h i},\left(\tilde{\theta}_{1}\right)_{h i}$, and $\pi-2\left(\tilde{\theta}_{1}\right)_{h i}$ and it follows that the angles between the diagonal of the rectangie and its sides are $\left(\tilde{\theta}_{1}\right)_{\mathrm{hi}}$ and $\pi / 2-\left(\tilde{\theta}_{1}\right)_{\mathrm{hi}}$.

The sides of the rectangle which lie along the axis of propagation of the signal are equal to $-\left(\Delta \epsilon_{\mathrm{h} 1}-\Delta \epsilon_{i 1}\right)$. in length. The length of the other two sides is initially unknown and is denoted by $T_{h i}$. The diagram also introduces a new angle $\left(\delta \theta_{1}\right)_{h i}$, which is the estimate of $\left(\Delta \theta_{1}\right)_{h i}$ produced by the approximation. 


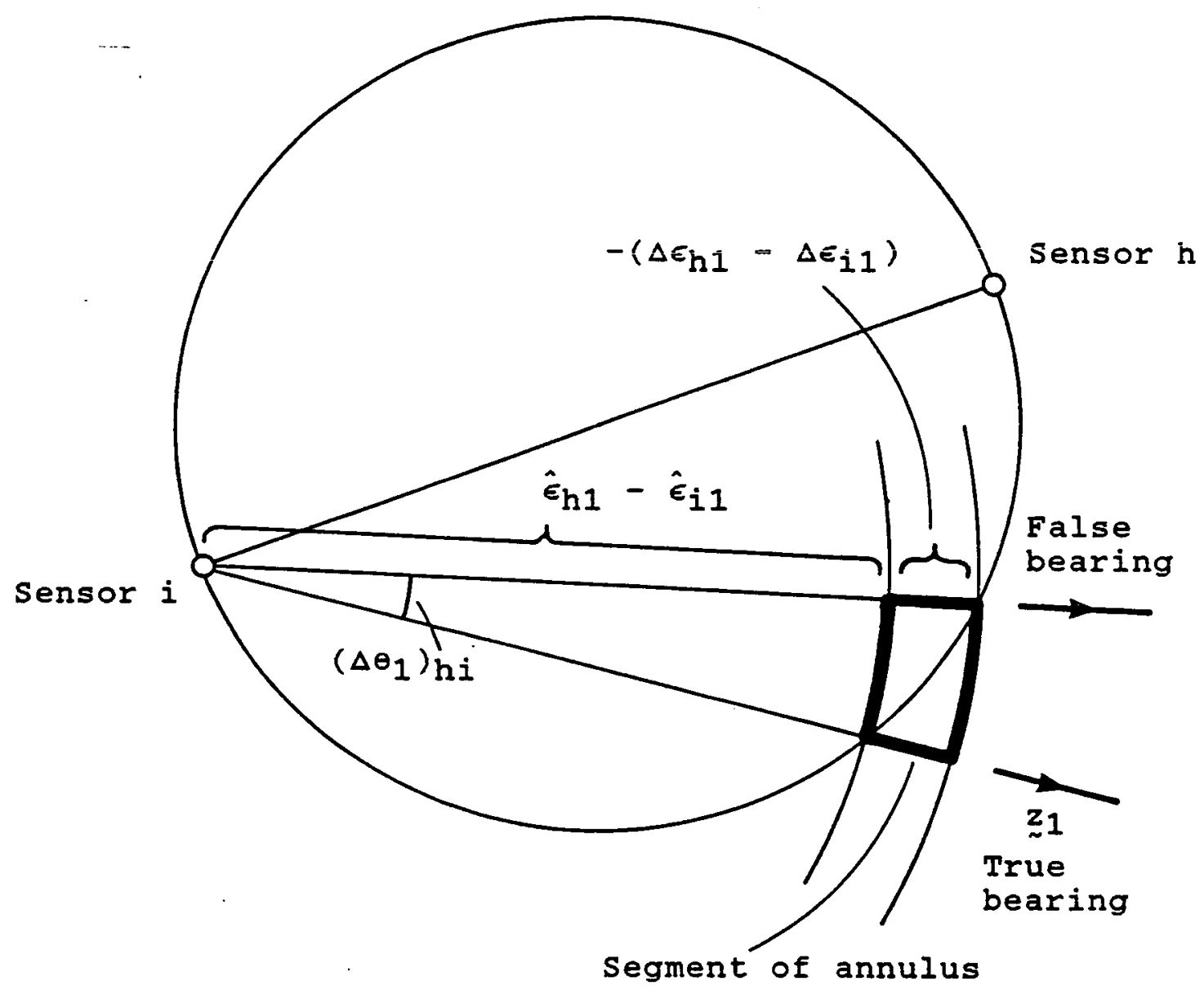

Figure 3.20 Segment of annulus 
(a)

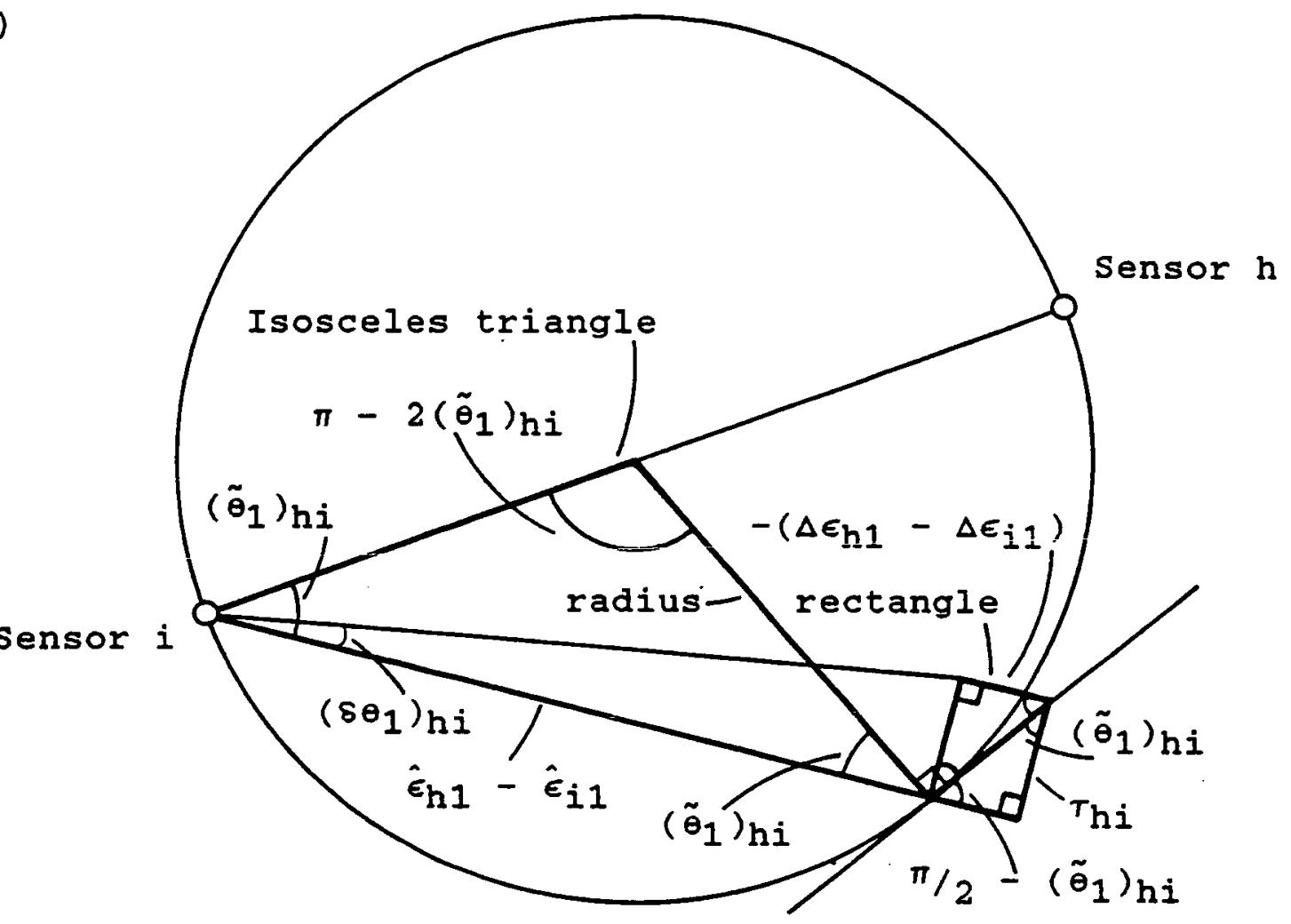

(b)

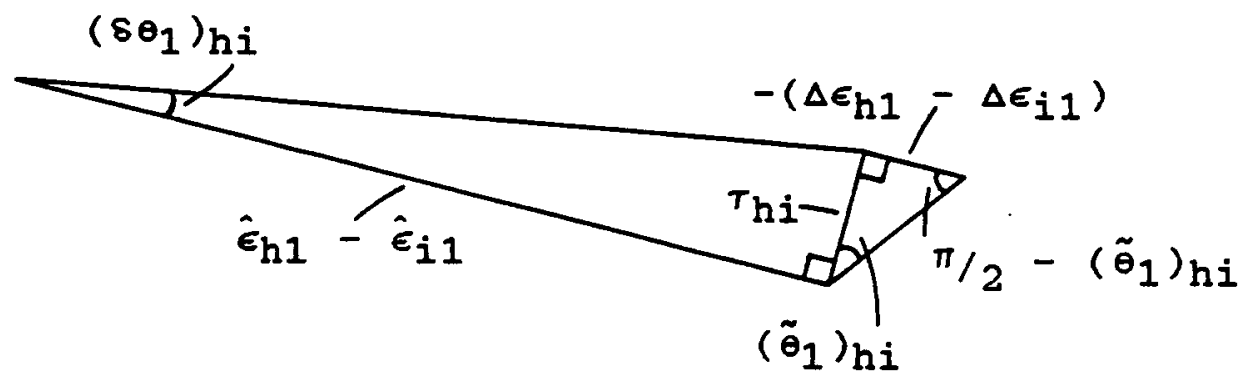

Figure 3.21 Graphical construction for approximate bearing error 
Figure $3.21(b)$ shows the elements of the construction required to solve for $\left(\delta \theta_{1}\right)_{h i}$. The first step is to find $T_{h i}$ by applying the sine rule in the small triangle. Notice that the sine of $\pi / 2-\left(\tilde{\theta}_{1}\right)_{h i}$ is equal to the cosine of $\left(\tilde{\theta}_{1}\right)_{\mathrm{hi}}$.

$$
\begin{aligned}
\frac{\Delta \epsilon_{\mathrm{h} 1}-\Delta \epsilon_{i 1}}{\sin \left[\left(\tilde{\theta}_{1}\right)_{h i}\right]} & =\frac{\tau_{h i}}{\sin \left[\pi / 2-\left(\tilde{\theta}_{I}\right)_{h i}\right]} \\
& =\frac{\tau_{h i}}{\cos \left[\left(\tilde{\theta}_{1}\right)_{h i}\right]}
\end{aligned}
$$

It is straightforward to obtain an expression for $\tau_{h i}$ by rearranging (3.32).

$$
\begin{aligned}
T_{h i} & =\frac{\left(\Delta \epsilon_{h 1}-\Delta \epsilon_{i 1}\right) \cos \left[\left(\tilde{\theta}_{1}\right)_{h i}\right]}{\sin \left[\left(\tilde{\theta}_{1}\right)_{h i}\right]} \\
& =\frac{\Delta \epsilon_{h 1}-\Delta \epsilon_{i 1}}{\tan \left[\left(\tilde{\theta}_{1}\right)_{h i}\right]}
\end{aligned}
$$

An expression for $\tan \left[\left(\tilde{\theta}_{1}\right)_{h i}\right]$ can be obtained from Figure 3.19 .

$$
\tan \left[\left(\tilde{\theta}_{1}\right)_{h i}\right]=\frac{v\left(\left\|\hat{\sim}_{h}-\hat{u}_{i}\right\|^{2}-\left(\hat{\epsilon}_{h 1}-\hat{\epsilon}_{i 1}\right)^{2}\right)}{\hat{\epsilon}_{h 1}-\hat{\epsilon}_{i 1}}
$$


Now substitute $(3.34)$ into $(3.33)$.

$$
T_{h i}=\frac{\left(\Delta \epsilon_{h 1}-\Delta \epsilon_{i 1}\right)\left(\hat{\epsilon}_{h 1}-\hat{\epsilon}_{i 1}\right)}{v\left\langle\left\|\hat{u}_{h}-\hat{u}_{i}\right\|^{2}-\left(\hat{\epsilon}_{h 1}-\hat{\epsilon}_{i 1}\right)^{2}\right\rangle}
$$

Returning to Figure $3.21(b)$, an expression for $\tan \left[\left(\delta \theta_{1}\right)_{h i}\right]$ can be obtained from the large triangle. since $\left(\delta \theta_{1}\right)_{h i}$ is a small angle, it is approximately equal to its own tangent.

$$
\left(\delta \theta_{1}\right)_{h i} \simeq \tan \left[\left(\delta \theta_{1}\right)_{h i}\right]=\frac{T_{h i}}{\hat{\epsilon}_{h 1}-\hat{\epsilon}_{i 1}}
$$

Substitute $(3.35)$ into $(3.36)$.

$$
\left(\delta \theta_{1}\right)_{h i}=\frac{\Delta \epsilon_{h 1}-\Delta \epsilon_{i 1}}{\checkmark\left(\left\|\hat{u}_{h}-\hat{u}_{i}\right\|^{2}-\left(\hat{\epsilon}_{h 1}-\hat{\epsilon}_{i 1}\right)^{2}\right)}
$$

Equation (3.37) is the approximation for $\left(\Delta \theta_{1}\right)_{\mathrm{hi}}$. The main advantage of this expression over (3.29) is that it is linearly proportional to $\Delta \epsilon_{\mathrm{h} 1}-\Delta \epsilon_{\mathrm{il}}$.

Figure 3.22 compares the precise expression with the approximation when $\left\|\hat{u}_{h}-\hat{u}_{i}\right\|^{2}=1$ and $\hat{\epsilon}_{h 1}-\hat{\epsilon}_{i 1}=0.5$. The angles $\left(\Delta \theta_{1}\right)_{h i}$ and $\left(\delta \theta_{1}\right)_{h i}$ are plotted as functions of $\Delta \epsilon_{\mathrm{hl}}-\Delta \epsilon_{\mathrm{il}}$, which ranges from -0.5 to 0.5 . The solid curve, which shows the true bearing error $\left(\Delta \theta_{1}\right)_{h i}$, was 


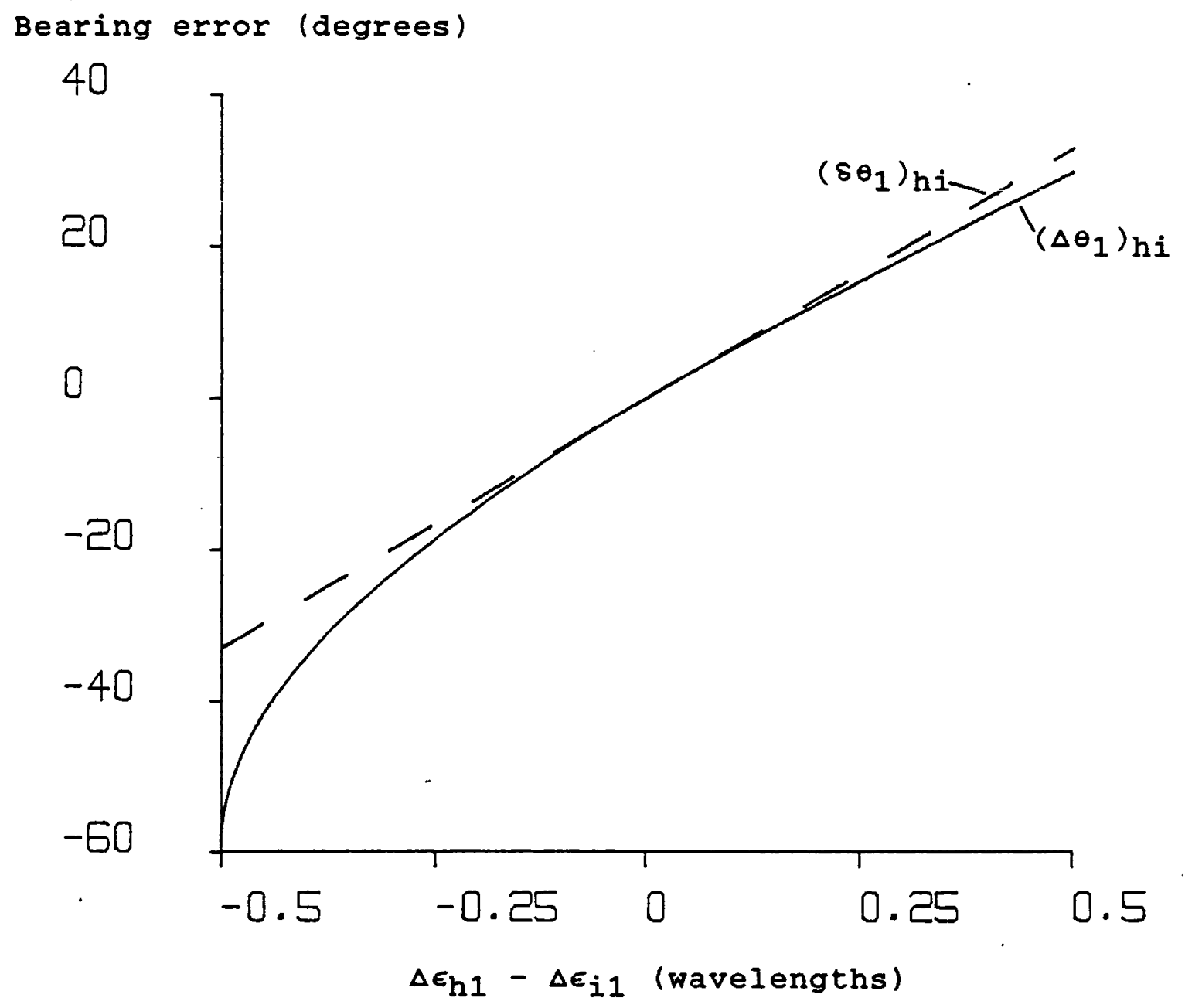

Figure 3.22 Comparison of precise bearing error $\left(\Delta \theta_{1}\right)_{h i}$ with approximation $\left(\delta \theta_{1}\right)_{h i}$ 
produced by finding $\sin \left[\left(\Delta \theta_{1}\right)_{\text {hi }}\right]$ using (3.29) then taking the inverse sine. The dashed line shows the approximation $\left(\delta \theta_{1}\right)_{\mathrm{hi}}$ produced using (3.37). The diagram confirms that $\left(\delta \theta_{1}\right)_{h i}$ is a good approximation to $\left(\Delta \theta_{1}\right)_{h i}$ when $\Delta \epsilon_{\mathrm{h} 1}-\Delta \epsilon_{\mathrm{il}}$ is small.

It is now possible to estimate the variance of $\left(\Delta \theta_{1}\right)_{h i}$. From (3.37), the variance of ( $\left.\delta \theta_{1}\right)_{h i}$ is as follows.

$$
\begin{aligned}
\mathrm{v}\left[\left(\delta \theta_{1}\right)_{\mathrm{hi}}\right] & =\mathrm{v}\left[\frac{\Delta \epsilon_{\mathrm{h} 1}-\Delta \epsilon_{i 1}}{v\left(\left\|\hat{\sim}_{\mathrm{h}}-\hat{u}_{i}\right\|^{2}-\left(\hat{\epsilon}_{\mathrm{h} 1}-\hat{\epsilon}_{i 1}\right)^{2}\right)}\right] \\
& =\frac{v\left[\Delta \epsilon_{\mathrm{h} 1}-\Delta \epsilon_{i 1}\right]}{\left\|\hat{u}_{\mathrm{h}}-\hat{\sim}_{i}\right\|^{2}-\left(\hat{\epsilon}_{\mathrm{h} 1}-\hat{\epsilon}_{i 1}\right)^{2}}
\end{aligned}
$$

Since $\Delta \epsilon_{\mathrm{h} 1}$ and $\Delta \epsilon_{\mathrm{i} 1}$ both have a normal distribution with a variance of $\sigma_{\mathrm{p}}^{2}$, the variance of $\Delta \epsilon_{\mathrm{h} 1}-\Delta \epsilon_{\mathrm{i} 1}$ is $2 \sigma_{\mathrm{p}}^{2}$.

$$
v\left[\left(\delta \theta_{1}\right)_{h i}\right]=\frac{2 \sigma_{p}^{2}}{\left\|\hat{u}_{h}-\hat{u}_{i}\right\|^{2}-\left(\hat{\epsilon}_{h 1}-\hat{\epsilon}_{i 1}\right)^{2}}
$$

$\Delta \theta_{1}$ is calculated according to (3.16) except that $\left(\Delta \theta_{1}\right)_{h i}$ is replaced with $\left(\delta \theta_{1}\right)_{h i}$. The variance of $\Delta \theta_{1}$ is therefore as follows. 


$$
\begin{aligned}
V\left[\Delta \theta_{1}\right] & =v\left[\frac{2}{N(N-1)} \sum_{h=1}^{N-1} \sum_{i=h+1}^{N}\left(\delta \theta_{1}\right)_{h i}\right] \\
& =\frac{4}{N^{2}(N-1)^{2}} \sum_{h=1}^{N-1} \sum_{i=h+1}^{N} v\left[\left(\delta \theta_{1}\right)_{h i}\right]
\end{aligned}
$$

The final expression for the variance of $\Delta \theta_{1}$ is obtained by substituting (3.39) into (3.40).

$$
v\left[\Delta \theta_{1}\right]=\frac{8 \sigma_{p}^{2}}{N^{2}(N-1)^{2}} \sum_{h=1}^{N-1} \sum_{i=h+1}^{N} \frac{1}{\left\|\hat{u}_{h}-\hat{u}_{i}\right\|^{2}-\left(\hat{\epsilon}_{h 1}-\hat{\epsilon}_{i 1}\right)^{2}}
$$

(3.41) shows that for small sensor positioning errors, the variance of the bearing error $\Delta \theta_{1}$ is directly proportional to the variance $\sigma_{\mathrm{p}}^{2}$ of the sensor positions. The variance of $\Delta \theta_{1}$ also depends on the number of sensors in the array, N. It is somewhat disappointing that (3.41) also makes reference to the array geometry (through $\hat{\sim}_{h}$ and $\hat{\sim}_{i}$ ) and the true bearing of the signal (through $\hat{\epsilon}_{\mathrm{h} 1}$ and $\hat{\epsilon}_{\mathrm{il}}$ ).

Figure 3.23 compares the simulation results with the curve predicted using (3.41) and there is evidently a close correspondence. As in the last section, the results produced by the three bearing estimation techniques are identical. 
(a) $v\left[\Delta \theta_{1}\right]$ (degrees squared)

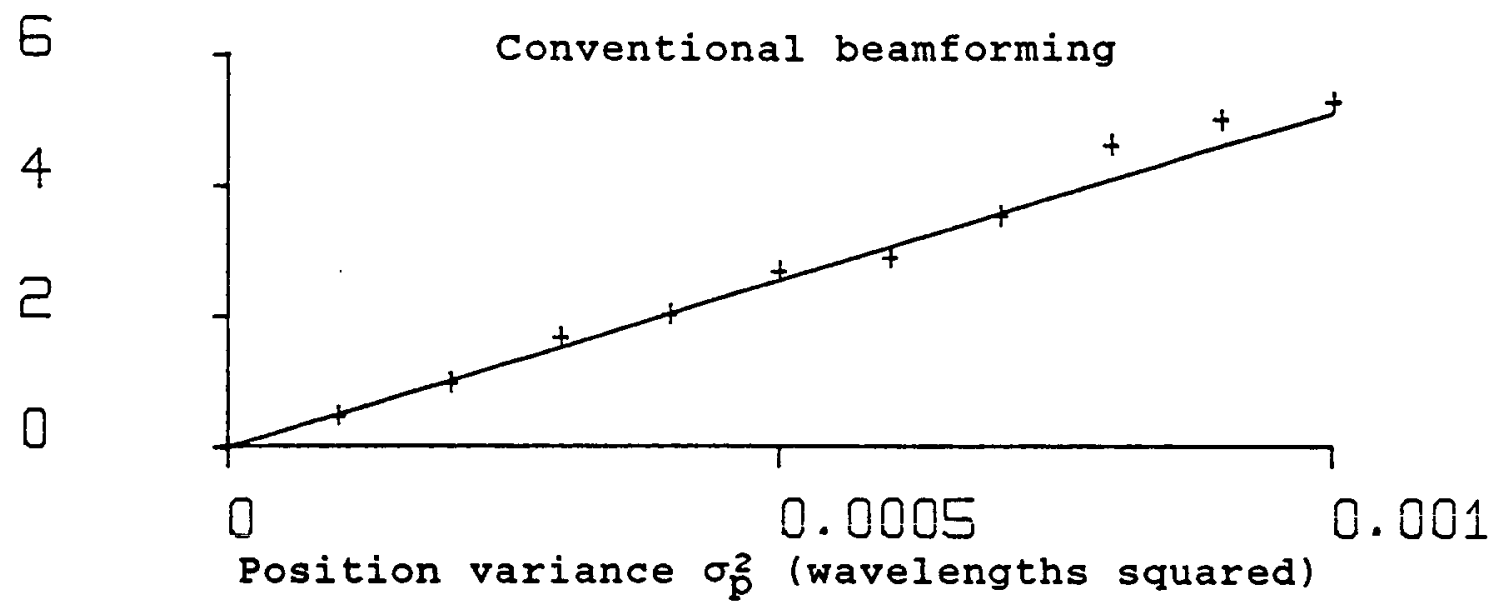

(b) $v\left[\Delta \theta_{1}\right]$ (degrees squared)

E

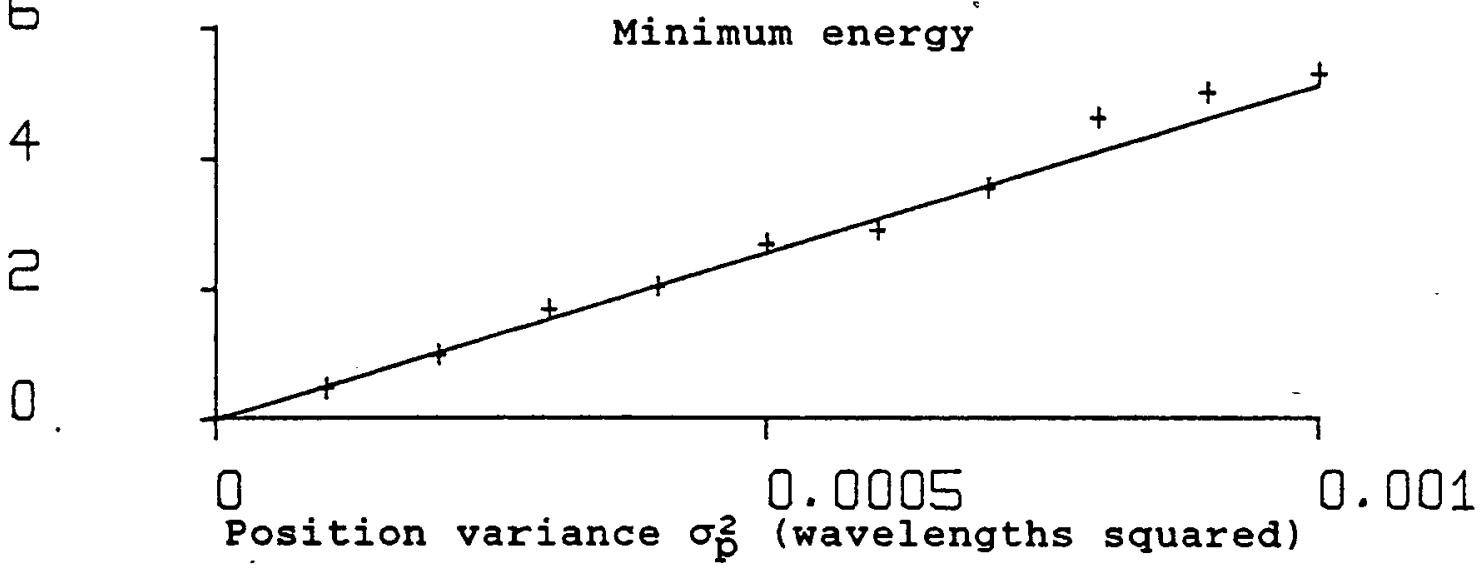

(c) $v\left[\Delta \theta_{1}\right]$ (degrees squared).

6

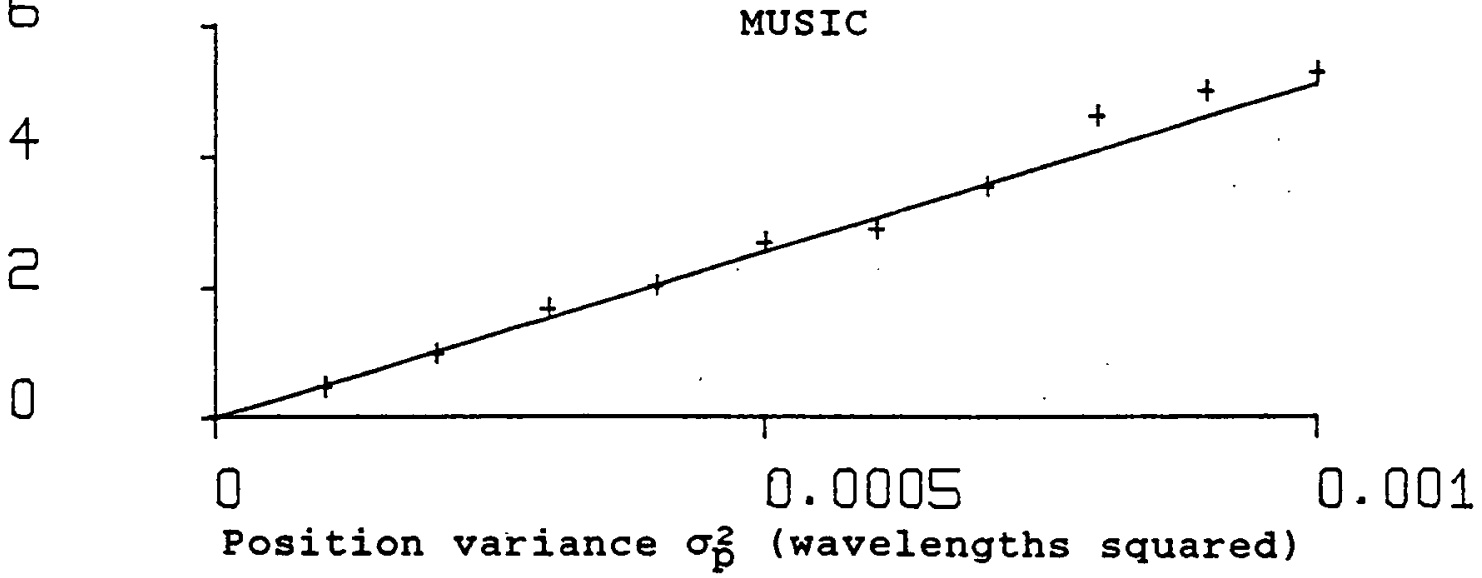

Figure 3.23 Results for variance of bearing error 
3.8 A General Quadratic Form for the spectrum Value at the True Bearing

In Chapter 2, the following expressions were derived for the bearing spectra produced by the conventional beamforming, minimum energy, and MUSIC bearing estimation techniques.

$$
\begin{aligned}
& \mathrm{P}_{\mathrm{bf}}(\theta)=\underline{\sim}^{\mathrm{g}}(\theta) \underset{\sim}{\mathrm{R}} \hat{\mathrm{q}}(\theta) \\
& \mathrm{P}_{\mathrm{me}}(\theta)=\frac{1}{{\underset{\sim}{\underline{q}}}^{\mathrm{H}}(\theta) \underset{\sim}{\mathrm{R}^{-1}} \underset{\sim}{\underline{q}}(\theta)} \\
& P_{m u}(\theta)=\frac{1}{\tilde{q}^{H}(\theta) \underline{w}_{W}{ }_{\sim}^{H} \hat{q}(\theta)}
\end{aligned}
$$

The remaining sections of this chapter are devoted to an algebraic analysis of the behaviour of $\mathrm{P}_{\mathrm{bf}}(\theta), \mathrm{P}_{\mathrm{me}}(\theta)$, and $\mathrm{P}_{\mathrm{mu}}(\theta)$ in the presence of sensor positioning errors. This leads to a set of theoretical expressions for the means and variances of $P_{b f}\left(\theta_{1}\right), P_{m e}\left(\theta_{1}\right)$, and $P_{m u}\left(\theta_{1}\right)$, which are the spectrum values obtained at the true bearing of the signal.

In the case of MUSIC, it is helpful to express $P_{m u}(\theta)$ in a slightly different form. Recall from Chapter 2 that the denominator in $(3.44)$ is equal to $\left\|\mathrm{p}_{w}(\theta)\right\|^{2}$, which is the 
length (squared) of the component of $\underset{\sim}{\hat{q}}(\theta)$ in the noise subspace. The component of $\hat{q}(\theta)$ in the signal subspace is denoted by $p_{s}(\theta)$ and, by Pythagoras' theorem, the sum of $\left\|p_{w}(\theta)\right\|^{2}$ and $\left\|p_{s}(\theta)\right\|^{2}$ must be equal to $\|\hat{q}(\theta)\|^{2}$. Since the $N$ elements of $\underset{q}{q}(\theta)$ all have unit magnitude, $\|\hat{q}(\theta)\|^{2}$ must be equal to $N$ and the denominator in $(3.44)$ can therefore be expressed as follows.

$$
\begin{aligned}
& \hat{q}^{H}(\theta){\underset{\sim}{W}}_{\sim} V_{W}^{H} \hat{q}(\theta)=\|\underset{\sim}{W}(\theta)\|^{2} \\
& =\|\hat{q}(\theta)\|^{2}-\left\|\underline{\sim}_{S}(\theta)\right\|^{2} \\
& =N-\left\|p_{S}(\theta)\right\|^{2} \\
& =N-\hat{q}^{H}(\theta) \underset{\sim}{V}{ }_{\sim} \underset{s}{H} \hat{q}(\theta)
\end{aligned}
$$

$V_{s}$ is a matrix which has the M signal subspace eigenvectors as its columns.

Setting $\theta$ equal to $\theta_{1}$ in the expressions for the bearing spectra given in $(3.42),(3.43)$, and $(3.45), \hat{q}(\theta)$ becomes equal to the signal vector $\hat{q}_{1}$ corresponding to the true bearing of the signal. The expressions used to investigate the performance of the three techniques at the bearing $\theta_{1}$ are as follows.

$$
\begin{aligned}
& \operatorname{P}_{\mathrm{bf}}\left(\theta_{1}\right)=\hat{q}_{1}^{H_{\sim}} \hat{\sim}_{q_{1}} \\
& \operatorname{P}_{\mathrm{me}}^{-1}\left(\theta_{1}\right)=\hat{q}_{1}^{H_{R}}{ }^{-1} \hat{q}_{1}
\end{aligned}
$$




$$
P-\frac{1}{m u}\left(\theta_{1}\right)=N-\hat{q}_{1}^{H} V_{S} v_{\sim}^{H} \hat{q}_{\mathcal{1}}
$$

Notice that (3.47) and (3.48) describe the reciprocals of $P_{m e}\left(\theta_{1}\right)$ and $P_{m u}\left(\theta_{1}\right)$, denoted by $P_{m e}^{-1}\left(\theta_{1}\right)$ and $P_{m u}^{-1}\left(\theta_{1}\right)$.. . The main reason for choosing to work with the reciprocal of the bearing spectrum rather than the spectrum itself is that the results obtained for the means and variances of $P_{m e}^{-1}\left(\theta_{1}\right)$ and $P_{m}^{-1}\left(\theta_{I}\right)$ do not couple through the reciprocal function. These results cannot be extended to $P_{m e}\left(\theta_{1}\right)$ and $\mathrm{P}_{\mathrm{mu}}\left(\theta_{1}\right)$ without resorting to inequalities.

Furthermore, $P_{m u}\left(\theta_{1}\right)$ is theoretically equal to infinity when there are no sensor positioning errors present as demonstrated in Chapter 2. The function is very unstable since a small increase in the value of the denominator in (3.44) causes a large decrease in the value of $P_{m u}\left(\theta_{1}\right)$. $\operatorname{P}_{\mathrm{mu}}^{-1}\left(\theta_{1}\right)$, however, is much more well-behaved, being theoretically equal to zero when there are no sensor positioning errors.

Notice that $(3.46),(3.47)$, and $(3.48)$ all contain quadratic forms in $\hat{\mathfrak{q}}_{1}$. In other words, all three equations contain an expression of the form $\hat{q}_{\sim} \mathrm{H}_{\sim} \hat{q}_{1}$, which is denoted by $P_{\Omega}\left(\theta_{1}\right)$.

$$
P_{\Omega}\left(\theta_{1}\right)=\hat{\hat{q}_{1}} \hat{H}_{\sim} \hat{q}_{1}
$$


The matrix $\underset{\sim}{\Omega}$ is equal to $\underset{\sim}{\mathrm{R}}, \underset{\sim}{\mathrm{R}}-1$, or $\underset{\sim}{\mathrm{V}} \underset{\sim}{\mathrm{V}} \mathrm{H}$ depending on which bearing estimation technique is being used.

$$
\begin{aligned}
& P_{b f}\left(\theta_{1}\right)=\hat{q}_{1}^{H_{1}} \Omega_{b f} \hat{q}_{1} \\
& \mathrm{P}_{\mathrm{me}}^{-1}\left(\theta_{1}\right)=\hat{q}_{1}^{H_{1}} \Omega_{\mathrm{me}} \hat{q}_{1} \\
& \mathrm{P}_{\mathrm{mu}}^{-1}\left(\theta_{1}\right)=\mathrm{N}=\hat{\mathrm{q}} \hat{\sim}_{1}^{\mathrm{H}} \hat{\sim}_{\mathrm{m}} \hat{\mathrm{q}}_{1}
\end{aligned}
$$

where

$$
\begin{aligned}
& \Omega_{\mathrm{bf}}=\underset{\sim}{\mathrm{R}} \\
& \Omega_{\mathrm{me}}=\underset{\sim}{\mathrm{R}}-1 \\
& \Omega_{\mathrm{mu}}={\underset{\sim}{\mathrm{S}}}_{\sim}{ }_{\sim}^{\mathrm{H}}
\end{aligned}
$$

The next three sections of this chapter discuss the effects of sensor positioning errors on the matrices $\Omega_{\mathrm{bf}}$. $\Omega_{\sim} \mathrm{me}$, and $\Omega_{\sim} \mathrm{mu}$. Following this, the mean and variance of $P_{\Omega}\left(\theta_{1}\right)$ are derived and the means and variances of $P_{b f}\left(\theta_{1}\right)$, $P_{\mathrm{me}}^{-1}\left(\theta_{1}\right)$ and $P_{\mathrm{mu}}^{-1}\left(\theta_{1}\right)$ are then obtained by substituting $\Omega_{b f}$, $\Omega_{\sim} \mathrm{me}$, or $\Omega_{\mathrm{mu}}$ for $\Omega$ as appropriate. 


\subsection{The Quadratic Form for Conventional Beamforming}

From the discussion earlier in this chapter, the lengths of the components of $\Delta{\underset{\sim}{n}}_{n}$ and $u_{n}$ in the direction of $z_{m}$ are $\Delta \epsilon_{\mathrm{nm}}$ and $\epsilon_{\mathrm{nm}}$. The phase shifts corresponding to $\Delta \epsilon_{\mathrm{nm}}$ and $\epsilon_{\mathrm{nm}}$ are denoted by $\Delta \psi_{\mathrm{nm}}$ and $\psi_{\mathrm{nm}}$ respectively and are calculated in the same way as $\hat{\psi}_{\mathrm{nm}}$.

$$
\begin{aligned}
& \Delta \psi_{n m}=2 \pi \Delta \epsilon_{n m} \\
& \psi_{n m}=2 \pi \epsilon_{n m}
\end{aligned}
$$

Recalling the relationship between $\epsilon_{\mathrm{nm}}, \hat{\epsilon}_{\mathrm{nm}}$, and $\Delta \epsilon_{\mathrm{nm}}$ given in (3.5), $\psi_{\mathrm{nm}}, \hat{\psi}_{\mathrm{nm}}$, and $\Delta \psi_{\mathrm{nm}}$ must be related to each other as follows.

$$
\Psi_{\mathrm{nm}}=\hat{\Psi}_{\mathrm{nm}}-\Delta \Psi_{\mathrm{nm}}+\Delta \Psi_{1 \mathrm{~m}}
$$

Like $\hat{\Psi}_{1 \mathrm{~m}}, \Psi_{1 \mathrm{~m}}$ is always zero and this can be confirmed by setting $n$ equal to 1 in $(3.58)$.

$$
\psi_{1 m}=\hat{\psi}_{1 \mathrm{~m}}=0
$$

A similar set of equations can be derived for the phasors corresponding to $\Delta \psi_{\mathrm{nm}}$ and $\Psi_{\mathrm{nm}}$. These are denoted by $\Delta q_{\mathrm{nm}}$ and $q_{n m}$ and are calculated as follows. 


$$
\Delta q_{n m}=\exp \left(j \Delta \Psi_{n m}\right)
$$

$$
q_{n m}=\exp \left\langle j \Psi_{n m}\right\rangle
$$

The relationship between $q_{n m}, \hat{q}_{n m}$, and $\Delta q_{n m}$ can be obtained by substituting (3.58) into (3.61). Since the phasors are all of unit magnitude, addition of phase shifts can be achieved by multiplying the corresponding phasors.

$$
\begin{aligned}
q_{n m} & =\exp \left(j\left(\hat{\Psi}_{n m}-\Delta \Psi_{n m}+\Delta \Psi_{1 m}\right)\right\} \\
& =\exp \left(j \hat{\Psi}_{n m}\right) \exp \left\langle-j \Delta \Psi_{n m}\right\} \exp \left\langle j \Delta \Psi_{1 m}\right) \\
& =\hat{q}_{n m} \Delta q_{n m}^{\star} \Delta q_{1 m}
\end{aligned}
$$

Like $\hat{q}_{1 \mathrm{~m}} \cdot q_{1 \mathrm{~m}}$ is always equal to 1 .

$$
\begin{aligned}
q_{1 \mathrm{~m}} & =\hat{q}_{1 \mathrm{~m}} \Delta q_{1 \mathrm{~m}}^{*} \Delta q_{1 \mathrm{~m}} \\
& =\hat{q}_{1 \mathrm{~m}}\left|\Delta q_{1 \mathrm{~m}}\right|^{2} \\
& =\hat{q}_{1 \mathrm{~m}} \\
& =1
\end{aligned}
$$

As explained in Chapter 2, the covariance matrix $\underset{\sim}{\mathrm{R}}$ is related to the bearings of the signals through the matrix

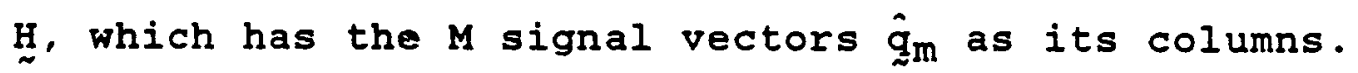

$$
\underset{\sim}{R}=\underset{\sim}{H} \underset{\sim}{R}{ }_{\sim}^{H} H+\sigma_{W}^{2} \underset{\sim}{I}
$$


When sensor positioning errors are present, $\hat{q}_{m}$ is replaced with the (unknown) signal vector corresponding to the true sensor positions. This vector is denoted by $q_{m}$ and is defined as follows.

$$
q_{n}=\left[\begin{array}{lllll}
1 & q_{2 m} & q_{3 m} & \ldots & q_{N m}
\end{array}\right]^{T}
$$

When there is only one signal present, $\underset{\sim}{\mathrm{H}}$ only has one column, this being the signal vector $q_{1}$. The signal correlation matrix $\underset{\sim a}{\mathrm{R}}$ is reduced to a one by one matrix whose only element is $\left(\underset{\sim}{\mathbb{R}_{\mathrm{a}}}\right)_{11}$. Recall from Chapter 2 that an element $\left(\underset{\sim}{\mathbb{R}_{a}}\right)_{\mathrm{mm}}$ on the leading diagonal of $\underset{\sim a}{\mathrm{R}_{a}}$ is equal to the power of signal $m$, denoted by $A_{m}^{2}$. It follows that $\left(\mathbb{R}_{a}\right)_{11}$ is equal to $A_{1}^{2}$. These observations allow (3.64) to be simplified as follows when there is only one signal present.

$$
\underset{\sim}{R}=A_{1}^{2} q_{1} q_{1}^{H}+\sigma_{W}^{2} I
$$

When $\underset{\sim}{R}$ is evaluated according to $(3.66)$, an element $R_{n n}$ on the leading diagonal can be expressed in terms of $A_{1}^{2}$ and $\sigma_{\mathrm{w}}^{2}$ as follows.

$$
\begin{aligned}
R_{n n} & =A_{1}^{2} q_{n 1} q_{n 1}^{*}+\sigma_{w}^{2} \\
& =A_{1}^{2}\left|q_{n 1}\right|^{2}+\sigma_{w}^{2} \\
& =A_{1}^{2}+\sigma_{W}^{2}
\end{aligned}
$$


An off-diagonal element $R_{h i}$ is as follows.

$$
R_{h i}=A_{1}^{2} q_{h 1} q_{i 1}^{*}, \quad h \neq i
$$

Combining (3.67) and (3.68), the definition of the matrix $\Omega_{b f}$, which is equal to $\underset{\sim}{R}$, can be written in the following form.

$$
\left(\Omega_{\mathrm{bf}}\right)_{\mathrm{hi}}=R_{\mathrm{hi}}= \begin{cases}\wedge_{\mathrm{bf}}, & \mathrm{h}=\mathrm{i} \\ \varsigma_{\mathrm{bf}} q_{\mathrm{h} 1} q_{i 1}^{*}, & \mathrm{~h} \neq i\end{cases}
$$

where

$$
\begin{aligned}
& \Lambda_{\mathrm{bf}}=\mathrm{A}_{1}^{2}+\sigma_{\mathrm{W}}^{2} \\
& s_{\mathrm{bf}}=\mathrm{A}_{1}
\end{aligned}
$$

\subsection{The Quadratic Form for Minimum Energy}

There is a matrix identity [16] which states that if a matrix is of the form $\underset{\sim}{R}=\underset{\sim}{S T \sim \sim} H+\underset{\sim}{T}$ then $i t s$ inverse is given by $\underset{\sim}{\mathrm{R}}-1=\underset{\sim}{\mathrm{I}}-\underset{\sim}{\mathrm{S}} \underset{\sim}{\mathrm{\sim}} \mathrm{H}$ where $\underset{\sim}{\mathrm{W}}=\left[\underset{\sim}{\mathrm{I}}+\underset{\sim}{\mathrm{T}} \mathrm{\sim}_{\sim}^{\mathrm{H}} \underset{\sim}{S}\right]^{-1} \underset{\sim}{\mathrm{T}}$. This can be used to gain some insight into the inverse of the covariance matrix $\underset{\sim}{R}$, which is required for the minimumn 
energy method. The first step is to divide both sides of $(3.66)$ by $\sigma_{\mathrm{w}}^{2}$

$$
\frac{1}{\sigma_{W}^{2}} \underset{\sim}{R}=\frac{A_{1}^{2}}{\sigma_{W}^{2}} q_{1} q_{1}^{H}+I
$$

Now apply the matrix identity, setting $\underset{\sim}{S}$ and $\underset{\sim}{U}$ equal to $\underset{\sim}{g_{1}} A_{1} / \sigma_{w}$ and $\underset{\sim}{T}$ equal to $\underline{\tau}$.

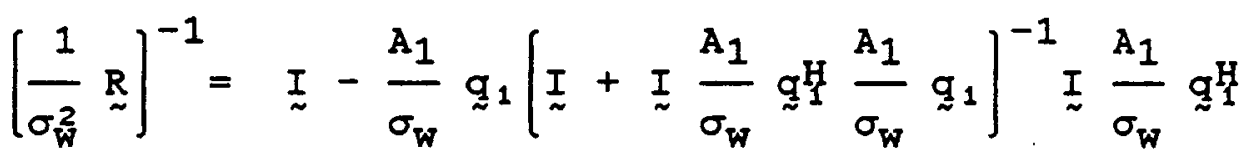

$$
\begin{aligned}
& =\quad I-\frac{A_{1}^{2}}{\sigma_{W}^{2}} q_{1}\left[\left[1+\left\|q_{1}\right\|^{2} \frac{A_{1}^{2}}{\sigma_{W}^{2}}\right] I\right]^{-1} \underset{\sim}{q_{1}^{H}} \\
& =\quad I-\frac{A_{1}^{2}}{\sigma_{W}^{2}} q_{1}\left[\left[1+\frac{N A_{1}^{2}}{\sigma_{W}^{2}}\right]\right]_{\sim}^{-1} \underset{\sim}{H} \\
& =I-\frac{A_{1}^{2}}{\sigma_{W}^{2}} q_{1} \frac{1}{1+N A_{1}^{2} / \sigma_{W}^{2}} \underset{\sim}{I} q_{1}^{H} \\
& =I-\frac{A_{1}^{2}}{\sigma_{W}^{2}+N A_{1}^{2}} q_{1} q_{1}^{H}
\end{aligned}
$$

The matrix $\mathrm{R}^{-1}$ is therefore as follows. 


$$
\stackrel{\sim}{R}^{-1}=\frac{1}{\sigma_{W}^{2}}\left[I-\frac{A_{1}^{2}}{\sigma_{W}^{2}+N A_{1}^{2}}{\underset{\sim}{q}}_{1} \underset{\sim}{H}\right]
$$

Thus an element $\left(R^{-1}\right)_{n n}$ on the leading diagonal of $R^{-1}$ can be expressed as follows.

$$
\begin{aligned}
\left(R^{-1}\right)_{n n} & =\frac{1}{\sigma_{W}^{2}}\left[1-\frac{A_{1}^{2}}{\sigma_{W}^{2}+N A_{1}^{2}} q_{n 1} q_{n 1}^{*}\right] \\
& =\frac{1}{\sigma_{W}^{2}}\left[1-\frac{A_{1}^{2}}{\sigma_{W}^{2}+N A_{1}^{2}}\left|q_{n 1}\right|^{2}\right] \\
& =\frac{1}{\sigma_{\mathrm{W}}^{2}}\left[1-\frac{A_{1}^{2}}{\sigma_{\mathrm{W}}^{2}+N A_{1}^{2}}\right]
\end{aligned}
$$

Similarly, an off-diagonal element $\left(R^{-1}\right)_{h i}$ has the following form.

$$
\left(R^{-1}\right)_{h i}=-\frac{A_{1}^{2}}{\sigma_{W}^{2}\left(\sigma_{W}^{2}+N A_{1}^{2}\right)} q_{h 1} q_{i}^{*}, \quad h \neq i
$$

The definition of the matrix $\Omega_{m e}$, which is equal to ${\underset{\sim}{R}}^{-1}$, is obtained by combining (3.75) and (3.76).

$$
\left(\Omega_{m e}\right)_{h i}=\left(R^{-1}\right)_{h i}= \begin{cases}\wedge_{m e}, & h=i \\ s_{m e} q_{h 1} q_{i 1}^{*}, & h \neq i\end{cases}
$$


where

$$
\begin{aligned}
& \wedge_{\mathrm{me}}=\frac{1}{\sigma_{W}^{2}}\left[1-\frac{A_{1}^{2}}{\sigma_{W}^{2}+N A_{1}^{2}}\right] \\
& S_{m e}=-\frac{A_{1}^{2}}{\sigma_{W}^{2}\left(\sigma_{W}^{2}+N A_{1}^{2}\right)}
\end{aligned}
$$

\subsection{The Quadratic Form for MUSIC}

As noted earlier, the matrix $\underset{\sim}{V}$ has the $M$ signal subspace eigenvectors as its columns. The $M$ signal vectors $q_{m}$ also span the signal subspace, although they are not normally collinear with the corresponding eigenvectors. However, the signal subspace is one-dimensional when there is only one signal present. In this case, Vs only contains the eigenvector $\mathrm{v}_{N}$, which must be collinear with the signal vector $q_{1}$.

$$
v_{s}=\underset{\sim}{N}=\frac{1}{\sqrt{N}} q_{1}
$$

The division by $\sqrt{N}$ is necessary because eigenvectors have unit length whereas signal vectors are of length $\sqrt{ }$. The the matrix product $\underset{\sim}{\mathrm{V}} \underset{\sim}{\mathrm{H}}$, which is required for MUSIC, is 
therefore as follows.

$$
\underset{\sim}{V_{s}} \underset{\sim}{V}=\frac{1}{\sqrt{N}} q_{\sim} \frac{1}{\sqrt{N}} q_{1}^{H}=\frac{1}{N} q_{1} q_{\sim}^{H}
$$

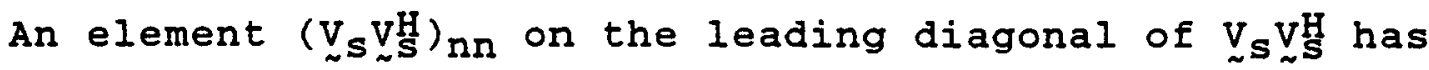
the following form.

$$
\left(v_{s} v_{s}^{H}\right)_{n n}=\frac{1}{N} q_{n 1} q_{n 1}^{*}=\frac{1}{N}\left|q_{n 1}\right|^{2}=\frac{1}{N}
$$

An off-diagonal element $\left(V_{\sim} \mathbb{\sim}_{\sim}^{\mathrm{V}}\right)_{h i}$ is as follows.

$$
\left(V_{s} \underset{\sim}{H}\right)_{h i}=\frac{1}{N} q_{h 1} q_{i 1}^{*}
$$

Combining (3.82) and (3.83), the definition of the matrix $\Omega_{\sim} \mathrm{mu}$, which is equal to ${\underset{\sim}{\mathrm{V}}}_{\sim} \underset{\sim}{\mathrm{V}}$, can be written as follows.

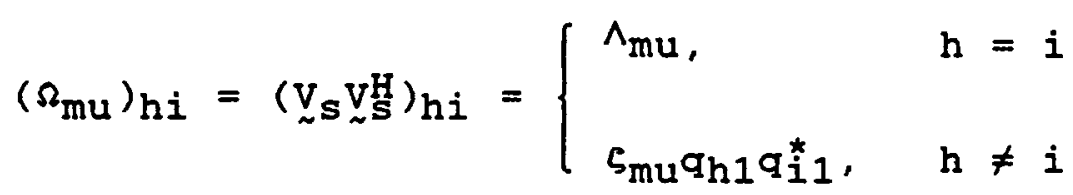

where

$$
\Lambda_{\mathrm{mu}}=\varsigma_{\mathrm{mu}}=\frac{1}{\overline{\mathrm{N}}}
$$




\subsection{The Mean of the Cosine of the Phase Error}

The expressions for $E\left[P_{\Omega}\left(\theta_{1}\right)\right]$ and $V\left[P_{\Omega}\left(\theta_{1}\right)\right]$ derived later in this chapter are found to contain references to $E\left[\cos \Delta \psi_{\mathrm{nm}}\right]$ and $E\left[\cos 2 \Delta \psi_{\mathrm{nm}}\right]$. This section relates the expected values of $\cos \Delta \Psi_{\mathrm{nm}}$ and $\cos 2 \Delta \Psi_{\mathrm{nm}}$ to the variance of the sensor positions $\sigma_{\mathrm{p}}^{2}$.

Since the cosine is a non-linear function, it is difficult to derive a precise relationship between the distribution of $\Delta \Psi_{n m}$ and that of $\cos \Delta \Psi_{n m}$ (or $\cos 2 \Delta \psi_{n m}$ ). A good approximation can, however, be obtained by using the series expansion of the cosine function up to the sixth order term [101].

$$
\begin{aligned}
& \cos \Delta \Psi_{n m} \simeq 1-\frac{\left(\Delta \Psi_{n m}\right)^{2}}{2 !}+\frac{\left(\Delta \Psi_{n m}\right)^{4}}{4 !}-\frac{\left(\Delta \Psi_{n m}\right)^{6}}{6 !} \\
& \cos 2 \Delta \Psi_{n m} \simeq 1-\frac{\left(2 \Delta \Psi_{n m}\right)^{2}}{2 !}+\frac{\left(2 \Delta \Psi_{n m}\right)^{4}}{4 !}-\frac{\left(2 \Delta \Psi_{n m}\right)^{6}}{6 !}
\end{aligned}
$$

For example, if $\Delta \Psi_{\mathrm{nm}}$ is $\pi / 2, \cos \Delta \Psi_{\mathrm{nm}}$ should be zero. When $\cos \Delta \psi_{\mathrm{nm}}$ is approximated using $(3.86)$, the result is $-0.001$

Expressions for $E\left[\cos \Delta \Psi_{\mathrm{nm}}\right]$ and $E\left[\cos 2 \Delta \psi_{\mathrm{nm}}\right]$ are obtained by taking the expected value of both sides of (3.86) and 
$(3.87)$.

$$
\begin{gathered}
E\left[\cos \Delta \psi_{n m}\right] \simeq 1-\frac{E\left[\left(\Delta \psi_{n m}\right)^{2}\right]}{2 !}+\frac{E\left[\left(\Delta \psi_{n m}\right)^{4}\right]}{4 !}-\frac{E[}{2 !}+\frac{16 E\left[\left(\Delta \Psi_{n m}\right)^{4}\right]}{4 !} \\
E\left[\cos 2 \Delta \psi_{n m}\right] \approx 1-\frac{4 E\left[\left(\Delta \Psi_{n m}\right)^{2}\right]}{2 !}+\frac{16}{6 !} \\
-\frac{64 E\left[\left(\Delta \Psi_{n m}\right)^{6}\right]}{6}
\end{gathered}
$$

Recall that the error $\Delta \epsilon_{\mathrm{nm}}$ has a normal distribution with a mean of zero and a variance of $\sigma_{\mathrm{p}}^{2}$. Since $\Delta \Psi_{\mathrm{nm}}$ is equal to $2 \pi \Delta \epsilon_{n m}, \Delta \Psi_{n m}$ has a normal distribution with a mean of zero and a variance of $4 \pi^{2} \sigma_{\mathrm{p}}^{2}$. The moment generating function [97] of $\Delta \Psi_{\mathrm{nm}}$, denoted by $\mathrm{G}(\mathrm{p})$, is therefore as follows.

$$
G(e)=\exp \left(2 \pi^{2} \sigma_{p}^{2} e^{2}\right\rangle
$$

The moment generating function allows the expected values of powers of $\Delta \psi_{\mathrm{nm}}$ to be obtained using the following relationstip.

$$
E\left[\left(\Delta \psi_{n m}\right)^{i}\right]=G(i) \cdot(0)
$$


$G(i)(0)$ denotes the $i^{\text {th }}$ derivative of $G(e)$ with respect to $p$ evalutated at $e=0$. The following expressions for $E\left[\left(\Delta \psi_{n m}\right)^{2}\right], E\left[\left(\Delta \psi_{n m}\right)^{4}\right]$, and $E\left[\left(\Delta \psi_{n m}\right)^{6}\right]$ are obtained through repeated differentiation of (3.90).

$$
\begin{aligned}
& E\left[\left(\Delta \psi_{n m}\right)^{2}\right]=G(2)(0)=4 \pi^{2} \sigma_{p}^{2} \\
& E\left[\left(\Delta \psi_{n m}\right)^{4}\right]=G(4)(0)=48 \pi^{4} \sigma_{p}^{4} \\
& E\left[\left(\Delta \psi_{n m}\right)^{6}\right]=G(6)(0)=960 \pi^{6} \sigma_{p}^{6}
\end{aligned}
$$

The approximations for $E\left[\cos \Delta \psi_{n m}\right]$ and $E\left[\cos 2 \Delta \psi_{n m}\right]$ are obtained by substituting (3.92), (3.93), and (3.94) into $(3.88)$ and $(3.89)$.

$$
E\left[\cos \Delta \psi_{n m}\right] \simeq 1-2 \pi^{2} \sigma_{p}^{2}+2 \pi^{4} \sigma_{p}^{4}-\frac{1}{3} \pi^{6} \sigma_{p}^{6}
$$

$$
E\left[\cos 2 \Delta \psi_{n m}\right]=1-8 \pi^{2} \sigma_{p}^{2}+32 \pi^{4} \sigma_{p}^{4}-\frac{256}{3} \pi^{6} \sigma_{p}^{6}(3.96)
$$

(3.95) and (3.96) can be checked (partially) by setting $\sigma_{p}^{2}$ to zero, in which case $E\left[\cos \Delta \psi_{\mathrm{nm}}\right]$ and $E\left[\cos 2 \Delta \psi_{\mathrm{nm}}\right]$ both evaluate to 1. This is to be expected since $\Delta \epsilon_{\mathrm{nm}}$ and $\Delta \psi_{n m}$ are always equal to zero when there are no sensor positioning errors present. 


\subsection{The Mean of the General Quadratic Form}

It has been shown that the matrices $\Omega_{\mathrm{bf}}, \Omega_{\sim} \mathrm{me}$, and $\Omega_{\sim} \mathrm{mu}$ all have the following general form.

$$
\Omega_{h i}= \begin{cases}\wedge, & h=i \\ \zeta q_{h 1} q_{i 1}^{*}, & h \neq i\end{cases}
$$

$\Lambda$ and $\zeta$ are set to $\Lambda_{\mathrm{bf}}$ and $\zeta_{\mathrm{bf}}, \Lambda_{\mathrm{me}}$ and $\zeta_{\mathrm{me}}$, or $\Lambda_{\mathrm{mu}}$ and $\zeta_{\mathrm{mu}}$ depending on which bearing estimation technique being used. This section derives an expression for the mean of $P_{\Omega}\left(\theta_{1}\right)$ in terms of $\wedge$ and $\zeta$ and it is then straightforward to obtain the means of $P_{b f}\left(\theta_{1}\right), P_{m e}^{-1}\left(\theta_{1}\right)$, and $P_{m u}^{-1}\left(\theta_{1}\right)$ by setting $\wedge$ and $\zeta$ to the appropriate values. The first step in finding the mean of $P_{\Omega}\left(\theta_{1}\right)$ is to expand the matrix multiplications in (3.49).

$$
\begin{aligned}
P_{\Omega}\left(\theta_{1}\right)= & \hat{q}_{i}^{H_{\Omega}} \hat{q}_{i} \\
& =\sum_{h=1}^{N} \sum_{i=1}^{N} \hat{q}_{h 1}^{k} \Omega_{h i} \hat{q}_{i 1}
\end{aligned}
$$

Notice that whenever $h=i$ in (3.98), a reference an element on the leading diagonal of $\Omega$ is produced. These elements can be isolated from the off-diagonal elements by re-writing $(3.98)$ as follows. 


$$
\begin{aligned}
P_{\Omega}\left(\theta_{1}\right) & =\sum_{h=1}^{N}\left\{\hat{q}_{h 1}^{*} \Omega_{h h} \hat{q}_{h 1}+\sum_{\substack{i=1 \\
i \neq h}}^{N} \hat{q}_{h 1}^{*} \Omega_{h i} \hat{q}_{i 1}\right\} \\
& =\sum_{h=1}^{N}\left\{\left|\hat{q}_{h 1}\right|^{2} \Omega_{h h}+\sum_{\substack{i=1 \\
i \neq h}}^{N} \hat{q}_{h 1}^{*} \Omega_{h i} \hat{q}_{i 1}\right\} \\
& =\sum_{h=1}^{N}\left\{\Omega_{h h}+\sum_{\substack{i=1 \\
i \neq h}}^{N} \hat{q}_{h 1}^{*} \Omega_{h i} \hat{q}_{i 1}\right\}
\end{aligned}
$$

The qualifier $i \neq h$ indicates that $i$ takes all values in the range 1 to $N$ except the current value of $h$ and it follows that $\Omega_{h i}$ in (3.99) always refers to an off-diagonal element. The next step is to substitute the definition of $\Omega$ given in $(3.97)$ into $(3.99)$.

$$
P_{\Omega}\left(\theta_{1}\right)=\sum_{h=1}^{N}\left\{\wedge+\sum_{\substack{i=1 \\ i \neq h}}^{N} s q_{h 1} q_{i 1}^{*} \hat{q}_{h 1}^{*} \hat{q}_{i 1}\right\}
$$

Recalling the definition of the phasor $q_{n m}$ given in (3.62), $q_{h 1}=\hat{q}_{h 1} \Delta q_{h 1}^{*} \Delta q_{11}$ and $q_{i 1}=\hat{q}_{i 1} \Delta q_{i 1}^{*} \Delta q_{11}$ and (3.100) can therefore be expanded as follows. 


$$
p_{\Omega}\left(\theta_{1}\right)=\sum_{h=1}^{N}\left\{\Lambda+5 \sum_{\substack{i=1 \\ i \neq h}}^{N} \hat{q}_{h 1} \Delta q_{h 1}^{*} \Delta q_{11} \hat{q}_{i 1}^{*} \Delta q_{i 1} \Delta q_{11}^{*} \hat{q}_{h 1}^{*} \hat{q}_{i 1}\right\}
$$$$
=\sum_{h=1}^{N}\left\{\Lambda+\zeta \sum_{\substack{i=1 \\ i \neq h}}^{N}\left|\hat{q}_{h 1}\right|^{2}\left|\hat{q}_{i 1}\right|^{2}\left|\Delta q_{11}\right|^{2} \Delta q_{h 1}^{*} \Delta q_{i 1}\right\}
$$$$
=\sum_{h=1}^{N}\left\{\Lambda+\zeta \sum_{\substack{i=1 \\ i \neq h}}^{N} \Delta q_{h 1}^{*} \Delta q_{i 1}\right\}
$$$$
=N \wedge+\zeta \sum_{h=1}^{N} \sum_{\substack{i=1 \\ i \neq h}}^{N} \Delta q_{h 1}^{*} \Delta q_{i 1}
$$

Consider the sequence of terms of the form $\Delta q_{h 1}^{\star} \Delta q_{i 1}$ produced by the nested summations in (3.101). If, for example, there is a term in which $h=3$ and $i=7$ there must also be a term in which $h=7$ and $i=3$. Thus each term $\Delta q_{h 1}^{\star} \Delta q_{i 1}$ is accompanied by its conjugate $\Delta q_{i 1}^{\star} \Delta q_{h 1}$. allowing $(3.101)$ to be re-written as follows.

$$
P_{\Omega}\left(\theta_{1}\right)=N \Lambda+5 \sum_{h=1}^{N-1} \sum_{i=h+1}^{N}\left\{\Delta q_{h 1}^{*} \Delta q_{i 1}+\Delta q_{i 1}^{*} \Delta q_{h 1}\right\}(3.102)
$$


Now, a complex number plus its own conjugate is equal to twice the real part of the number.

$$
\Delta q_{h 1}^{\star} \Delta q_{i 1}+\Delta q_{i 1}^{\star} \Delta q_{h 1}=2 \operatorname{Re}\left[\Delta q_{h 1}^{\star} \Delta q_{i 1}\right]
$$

Recall that $\Delta q_{h 1}$ and $\Delta q_{i 1}$ both represent phasors of unit length. The phase angles of $\Delta q_{h 1}^{\star}$ and $\Delta q_{i 1}$ are $-\Delta \psi_{h 1}$ and $\Delta \psi_{i l}$ respectively, so the product $\Delta q_{h 1}^{\dot{k}} \Delta q_{i l}$ represents a phasor with unit length and phase angle $\Delta \psi_{i 1}-\Delta \psi_{h 1}$. It follows that the real part of $\Delta q_{h 1}^{\star} \Delta q_{i 1}$ is equal to the cosine of $\Delta \psi_{i 1}-\Delta \psi_{h 1}$.

$$
\operatorname{Re}\left[\Delta q_{h 1}^{*} \Delta q_{i 1}\right]=\cos \left(\Delta \psi_{i 1}-\Delta \psi_{h 1}\right)
$$

The expression for $P_{\Omega}\left(\theta_{1}\right)$ can now be simplified by substituting (3.103) and (3.104) into (3.102).

$$
P_{\Omega}\left(\theta_{1}\right)=N \Lambda+25 \sum_{h=1}^{N-1} \sum_{i=h+1}^{N} \cos \left(\Delta \psi_{i 1}-\Delta \psi_{h 1}\right)
$$

The expected value of $P_{\Omega}\left(\theta_{1}\right)$ is therefore as follows.

$$
E\left[P_{\Omega}\left(\theta_{1}\right)\right]=N \wedge+25 \sum_{h=1}^{N-1} \sum_{i=h+1}^{N} E\left[\cos \left(\Delta \psi_{i 1}-\Delta \psi_{h 1}\right)\right]
$$


The expected value of $\cos \left(\Delta \psi_{i 1}-\Delta \psi_{h 1}\right)$ can be expanded using a double-angle formula.

$$
\begin{aligned}
E\left[\cos \left(\Delta \psi_{i 1}-\Delta \psi_{\mathrm{h} 1}\right)\right]= & E\left[\cos \Delta \psi_{i 1} \cos \Delta \psi_{\mathrm{h} 1}\right. \\
& \left.+\sin \Delta \psi_{i 1} \sin \Delta \psi_{\mathrm{h} 1}\right]
\end{aligned}
$$

Since $h$ and $i$ are always different, $\Delta \psi_{\mathrm{h} 1}$ and $\Delta \psi_{i 1}$ are independent random variables and (3.107) can therefore be re-written as follows.

$$
\begin{aligned}
E\left[\cos \left(\Delta \psi_{i 1}-\Delta \psi_{h 1}\right)\right] & =E\left[\cos \Delta \psi_{i 1}\right] E\left[\cos \Delta \psi_{h 1}\right] \\
& +E\left[\sin \Delta \psi_{i 1}\right] E\left[\sin \Delta \psi_{h 1}\right]
\end{aligned}
$$

Now, $\Delta \psi_{\text {h1 }}$ has a normal distribution with a mean of zero, so a particular value of $\Delta \psi_{h 1}$, say $e$, has the same probability of occurring as $-\rho$. Since $\sin (-\rho)=-\sin (e)$, $E\left[\sin \Delta \psi_{\mathrm{hl}}\right]$ must be equal to zero. The same argument applies to $E\left[\sin \Delta \psi_{i 1}\right]$. Thus the sine terms in (3.108) vanish.

$$
E\left[\cos \left(\Delta \psi_{i 1}-\Delta \psi_{h 1}\right)\right]=E\left[\cos \Delta \psi_{i 1}\right] E\left[\cos \Delta \psi_{h 1}\right]
$$

The expression for $P_{\Omega}\left(\theta_{1}\right)$ can be simplified by substituting (3.109) into (3.106). 


$$
E\left[P_{\Omega}\left(\theta_{1}\right)\right]=N \Lambda+2 \zeta \sum_{h=1}^{N-1} \sum_{i=h+1}^{N} E\left[\cos \Delta \psi_{i 1}\right] E\left[\cos \Delta \psi_{h 1}\right]
$$

Since the angles $\Delta \psi_{h 1}$ and $\Delta \psi_{i 1}$ have the same statistics as the general angle $\Delta \Psi_{\mathrm{nm}}, E\left[\cos \Delta \Psi_{\mathrm{h} 1}\right]$ and $E\left[\cos \Delta \Psi_{i 1}\right]$ are both equal to $E\left[\cos \Delta \psi_{\mathrm{nm}}\right]$.

$$
\begin{aligned}
E\left[P_{\Omega}\left(\theta_{1}\right)\right] & =N \wedge+25 \sum_{h=1}^{N-1} \sum_{i=h+1}^{N}\left(E\left[\cos \Delta \psi_{n m}\right]\right)^{2} \\
& =N \Lambda+N(N-1) s\left(E\left[\cos \Delta \psi_{n m}\right]\right)^{2}
\end{aligned}
$$

\subsection{The Mean of the spectrum Value at the True Bearing}

From (3.49), $P_{\Omega}\left(\theta_{1}\right)$ is the quadratic form $\hat{q}_{1}^{H} \Omega_{\sim} \hat{q}_{1}$, which appears in the definitions of $\mathrm{P}_{\mathrm{bf}}\left(\theta_{1}\right), \mathrm{P}_{\mathrm{me}}^{-1}\left(\theta_{1}\right)$, and $\mathrm{P}_{\mathrm{mu}}^{-1}\left(\theta_{1}\right)$ given in $(3.50),(3.51)$, and (3.52). The matrix $\Omega$ is set to $\Omega_{\sim} \mathrm{bf}, \Omega_{\mathrm{me}}$, or $\Omega_{\sim} \mathrm{mu}$ as appropriate. From (3.97), $\Omega$ is characterized by by $\wedge$ and 5 . For example, $\Omega$ is set to $\Omega_{\text {bf }}$ by setting $\wedge$ equal to $\Lambda_{b f}$ and $\zeta$ equal to $\zeta_{\mathrm{bf}}$. The expected values of $\mathrm{P}_{\mathrm{bf}}\left(\theta_{1}\right), \mathrm{P}_{\mathrm{me}}^{-1}\left(\theta_{1}\right)$, and $P_{\mathrm{mu}}^{-1}\left(\theta_{1}\right)$ can therefore be expressed in terms of the expected value of $P_{\Omega}\left(\theta_{1}\right)$. 


$$
\begin{aligned}
& E\left[P_{b f}\left(\theta_{1}\right)\right]=E\left[P_{\Omega}\left(\theta_{1}\right)\right], \quad \Lambda=\Lambda_{b f}, \zeta=S_{b f} \text { (3.112) } \\
& E\left[P_{m e}^{-1}\left(\theta_{1}\right)\right]=E\left[P_{\Omega}\left(\theta_{1}\right)\right], \quad \Lambda=\Lambda_{\text {me }}, \quad s=\zeta_{\text {me }} \quad \text { (3.113) } \\
& E\left[P_{\mathrm{mu}}^{-1}\left(\theta_{1}\right)\right]=N-E\left[P_{\Omega}\left(\theta_{1}\right)\right], \quad \Lambda=\Lambda_{\mathrm{mu}}, \zeta=\zeta_{\mathrm{mu}}, \ldots
\end{aligned}
$$

Now, an expression for $E\left[P_{\Omega}\left(\theta_{1}\right)\right]$ in terms of $\wedge$ and $\zeta$ is given in (3.111) and $\Lambda_{\mathrm{bf}}, \zeta_{\mathrm{bf}}, \Lambda_{\mathrm{me}}, \zeta_{\mathrm{me}}, \Lambda_{\mathrm{mu}}$, and $\zeta_{\mathrm{mu}}$ are defined by $(3.70),(3.71),(3.78),(3.79)$, and $(3.85)$. The expected values of $P_{b f}\left(\theta_{1}\right), P_{m e}^{-1}\left(\theta_{1}\right)$, and $P_{m u}^{-1}\left(\theta_{1}\right)$ are therefore as follows.

$$
\begin{aligned}
& E\left[P_{b f}\left(\theta_{1}\right)\right]=N\left(A_{1}^{2}+\sigma_{W}^{2}\right)+N(N-1) A_{1}^{2}\left(E\left[\cos \Delta \psi_{n m}\right]\right)^{2} \\
& E\left[P_{m e}^{-1}\left(\theta_{1}\right)\right]=\frac{N}{\sigma_{W}^{2}}\left[1-\frac{A_{1}^{2}}{\sigma_{W}^{2}+N A_{1}^{2}}\right] \\
& -\frac{N(N-1) A_{1}^{2}}{\sigma_{W}^{2}\left(\sigma_{W}^{2}+N A_{1}^{2}\right)}\left(E\left[\cos \Delta \psi_{n m}\right]\right)^{2} \\
& E\left[P_{\mathrm{mu}}^{-1}\left(\theta_{1}\right)\right]=(N-1)\left(1-\left(E\left[\cos \Delta \Psi_{\mathrm{nm}}\right]\right)^{2}\right)
\end{aligned}
$$

The expected values of $P_{b f}\left(\theta_{1}\right), P_{m}^{-1}\left(\theta_{1}\right)$, and $P_{m}^{-1}\left(\theta_{1}\right)$ are related to the variance of the sensor positions $\sigma_{p}^{2}$ through (3.95), which defines $E\left[\cos \Delta \psi_{\mathrm{nm}}\right]$ in terms of $\sigma_{\mathrm{p}}^{2}$. 
Figure 3.24 shows the close agreement between the simulation results and the curves predicted using (3.115), (3.116), and (3.117). Recalling that the results for minimum energy and MUSIC correspond to the reciprocals of $P_{m e}(\theta)$ and $P_{m u}(\theta)$, it is evident that $E\left[P_{b f}(\theta)\right]$, $E\left[P_{m e}(\theta)\right]$, and $E\left[P_{m u}(\theta)\right]$ all decrease as $\sigma_{p}^{2}$ increases.

The effect is most pronouñed when the minimum energy and MUSIC techniques are used. When $\sigma_{p}^{2}=0, E\left[P_{m}^{-1}\left(\theta_{1}\right)\right]$ and $E\left[P_{m}^{-1}\left(\theta_{1}\right)\right]$ are both close to zero and $E\left[P_{m e}(\theta)\right]$ and $E\left[P_{m u}(\theta)\right]$ must therefore have large values. As $\sigma_{p}^{2}$ is increased, $E\left[P_{m e}^{-1}\left(\theta_{1}\right)\right]$ and $E\left[P_{m u}^{-1}\left(\theta_{1}\right)\right]$ both increase and $E\left[P_{m e}(\theta)\right]$ and $E\left[P_{m u}(\theta)\right]$ decrease rapidly since the gradient of the reciprocal function has its largest (negative) value in this area.

It should be stressed, however, that there is no simple quantitative relationship between $E\left[P_{m e}\left(\theta_{1}\right)\right]$ and $E\left[P_{m e}^{-1}\left(\theta_{1}\right)\right]$. In particular, $E\left[P_{m e}\left(\theta_{1}\right)\right] \neq 1 / E\left[P_{m e}^{-1}\left(\theta_{1}\right)\right]$ since the reciprocal function distorts the distribution of $\mathrm{P}_{\mathrm{me}}\left(\theta_{1}\right)[97]$. The same applies to $E\left[\mathrm{P}_{\mathrm{mu}}(\theta)\right]$ and $E\left[P-\frac{1}{\operatorname{mu}}\left(\theta_{1}\right)\right]$.

When there are no sensor positioning errors, there are no errors in the bearings of the peaks in the spectra. In this case, $\operatorname{P}_{b f}\left(\theta_{1}\right), P_{m e}^{-1}\left(\theta_{1}\right)$, and $P_{m}^{-1}\left(\theta_{1}\right)$ describe the peak heights (or their reciprocals). These values are 
(a)

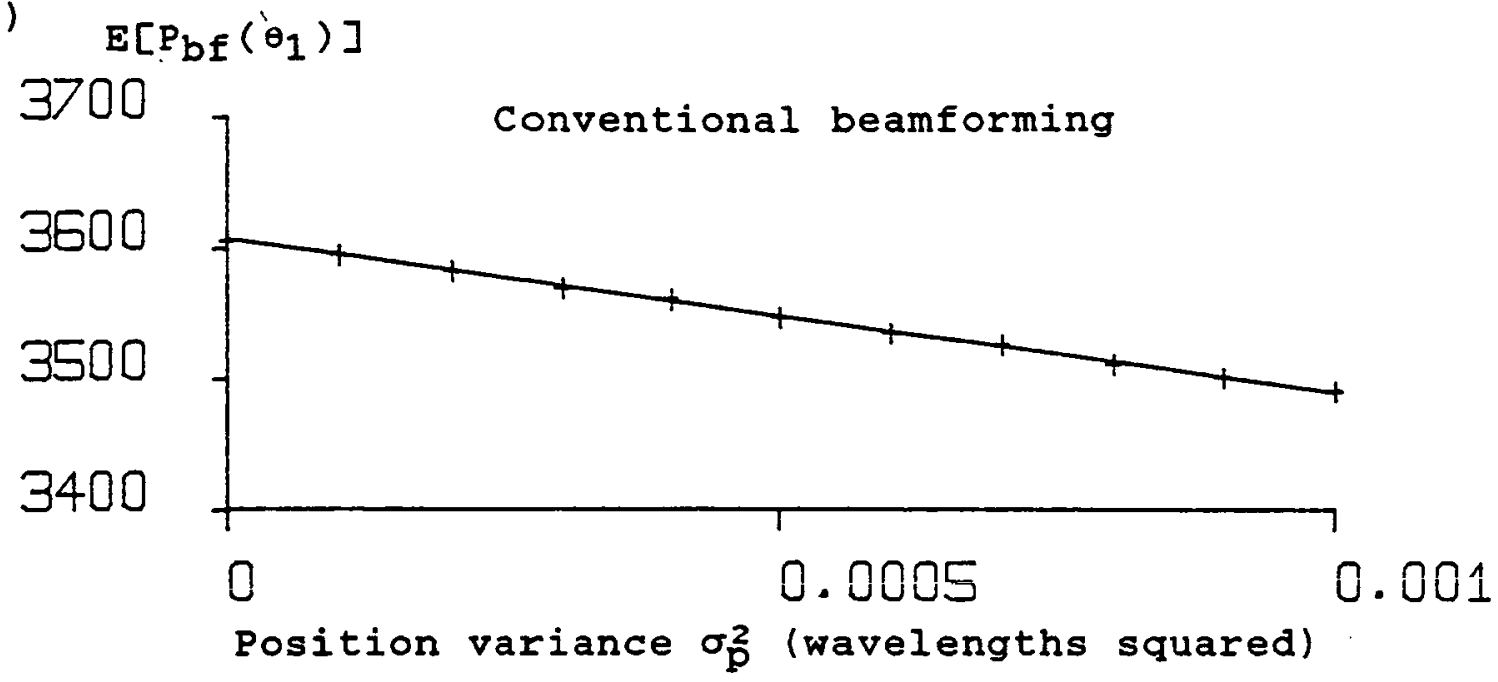

(b) $\quad E\left[P_{m e}^{-1}\left(\theta_{1}\right)\right]$
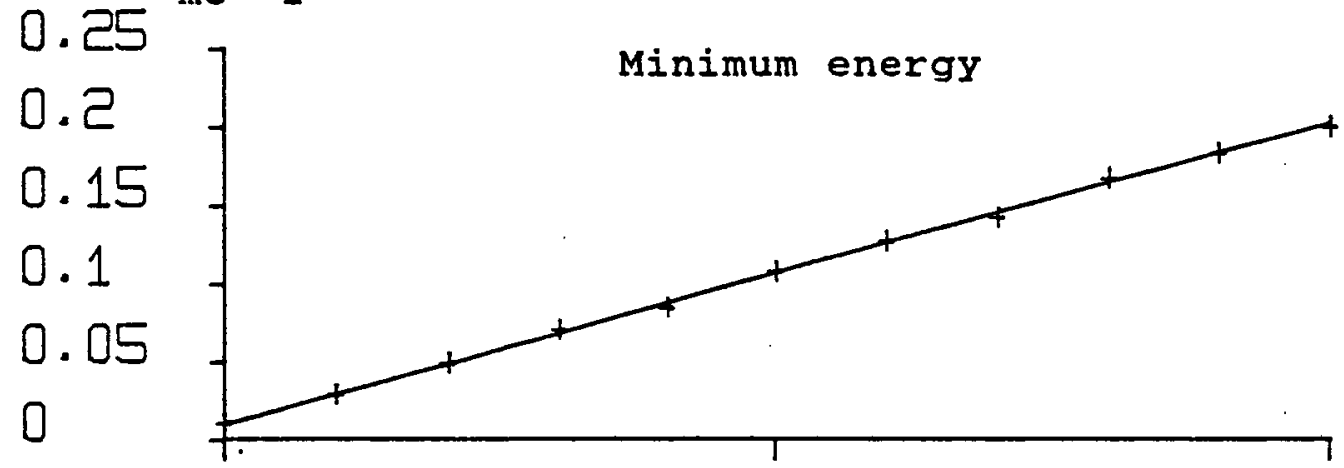

$\begin{array}{ll}0 & 0.0005 \\ \text { Position variance } \sigma_{0}^{2} \text { (wavelengths squared) }\end{array}$

(c) $\quad E\left[P^{-1} \mathfrak{m}\left(\theta_{1}\right)\right]$
0.25
0.2
0.15
0.1
0.05
0

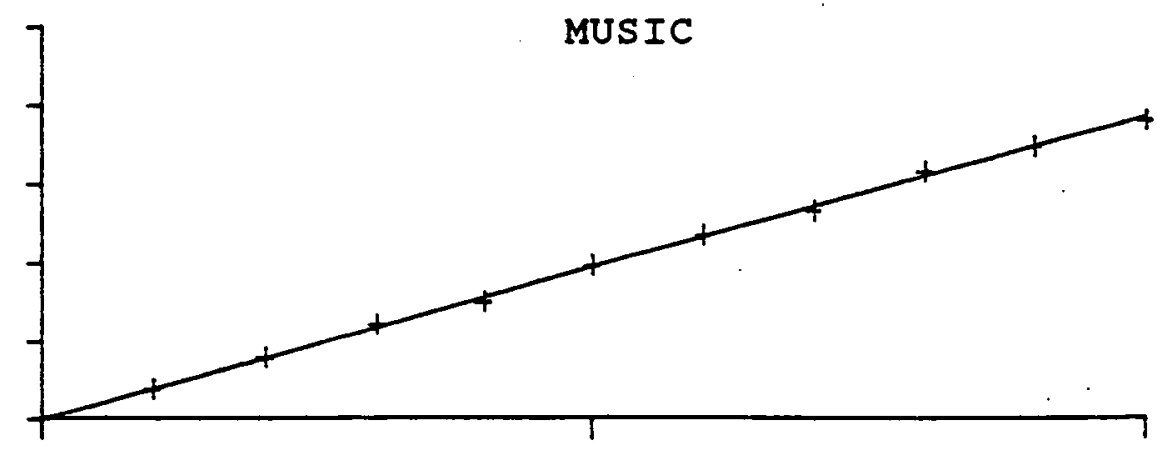

0

0.0005

0.001

Position variance $\sigma_{p}^{2}$ (wavelengths squared)

Figure 3.24 Results for mean of spectrum height at
true bearing 
calculated by evaluating (3.115), (3.116), and (3.117)

with $\sigma_{p}^{2}$ set to zero. From (3.95), $E\left[\cos \Delta \psi_{n m}\right]=1$ when $\sigma_{p}^{2}$ is zero.

$$
\begin{aligned}
& P_{b f}\left(\theta_{1}\right)=N^{2} A_{1}^{2}+N \sigma_{W}^{2}, \quad \sigma_{p}^{2}=0 \\
& P_{m}^{-1}\left(\theta_{1}\right)=\frac{N}{\sigma_{W}^{2}+N A_{1}^{2}}, \quad \sigma_{D}^{2}=0 \\
& P_{\mathrm{mu}}^{-1}\left(\theta_{1}\right)=0, \quad \sigma_{\mathrm{p}}^{2}=0
\end{aligned}
$$

The expressions given in (3.118), (3.119), (3.120) are in agreement with results published elsewhere [16]. Notice that $P_{b f}\left(\theta_{1}\right)$ and the reciprocal of $P_{m e}^{-1}\left(\theta_{1}\right)$ are both linearly proportional to the signal power $A_{1}^{2}$ and the noise power $\sigma_{W}^{2}$, thus confirming the statement made in Chapter 2 that conventional beamforming and minimum energy both produce bearing spectra which have units of power.

\subsection{The Variance of the General Quadratic Form}

This section derives an expression for the variance of $P_{\Omega}\left(\theta_{1}\right)$, which is then used in the next section to obtain the variances of $P_{b f}\left(\theta_{1}\right), P_{m e}^{-1}\left(\theta_{1}\right)$, and $P_{m u}^{-1}\left(\theta_{1}\right)$. The first step is to produce expressions for $\left(P_{\Omega}\left(\theta_{1}\right)\right\rangle^{2}$ and 
hence the mean square value $E\left[\left(P_{\Omega}\left(\theta_{1}\right)\right)^{2}\right]$. From the expression for $P_{\Omega}\left(\theta_{1}\right)$ given in $(3.101),\left\langle P_{\Omega}\left(\theta_{1}\right)\right\rangle^{2}$ is as follows.

$$
\begin{aligned}
\left\langle P_{\Omega}\left(\theta_{1}\right)\right\rangle^{2} & =\left\{N \Lambda+s \sum_{h=1}^{N} \sum_{\substack{i=1 \\
i \neq h}}^{N} \Delta q_{h 1}^{*} \Delta q_{i 1}\right\}^{2} \\
& =N^{2} \Lambda^{2}+2 N \wedge s \sum_{h=1}^{N} \sum_{\substack{i=1 \\
i \neq h}}^{N} \Delta q_{h 1}^{*} \Delta q_{i 1} \\
& +\varsigma^{2} \sum_{h=1}^{N} \sum_{\substack{i=1 \\
i \neq h}}^{N}\left[q_{h 1}^{*} \Delta q_{i 1} \sum_{p=1}^{N} \sum_{\substack{r=1 \\
r \neq p}}^{N} \Delta q_{p 1}^{*} \Delta q_{r 1}\right]
\end{aligned}
$$

The nested summations in the square brackets must be treated with care. Although there are some terms in which $h, i, p$, and $r$ are all different, there are several terms in which $p$ and/or $r$ duplicates $h$ and/or $i$.

As in (3.99), the approach is to re-write the summations so that the special terms are isolated. 


$$
\left\langle P_{\Omega}\left(\theta_{1}\right)\right\rangle^{2}=N^{2} \Lambda^{2}+2 N \wedge \zeta \sum_{h=1}^{N} \sum_{\substack{i=1 \\ i \neq h}}^{N} \Delta q_{h 1}^{k} \Delta q_{i 1}
$$$$
+\varsigma^{2} \sum_{h=1}^{N} \sum_{\substack{i=1 \\ i \neq h}}^{N}\left[\left(\Delta q_{h 1}^{*} \Delta q_{i 1}\right)^{2}+1+\sum_{\substack{p=1 \\ p \neq h \\ p \neq i}}^{N}\left\{\left(\Delta q_{h 1}^{*}\right)^{2} \Delta q_{i 1} \Delta q_{p 1}\right.\right.
$$$$
\left.+\Delta q_{h 1}^{\star}\left(\Delta q_{i 1}\right)^{2} \Delta q_{p 1}^{\star}+\Delta q_{h 1}^{\star} \Delta q_{p 1}+\Delta q_{i 1} \Delta q_{p 1}^{\star}\right\}
$$

$$
\left.+\sum_{\substack{p=1 \\ p \neq h}}^{N} \sum_{\substack{r=1 \\ p \neq i}}^{N \neq h} \Delta q_{h 1}^{k} \Delta q_{i 1} \Delta q_{p 1}^{*} \Delta q_{r 1}\right]
$$

It is straightforward to show that (3.122) evaluates to a real number, the argument being the same as the one which was applied to (3.101). Consider the two summations in which $h$ and $i$ run from 1 to $N$. If there is a step at which, say, $h=3$ and $i=7$, there must also be a step at which $\mathrm{h}=7$ and $i=3$. The terms produced when $\mathrm{h}=7$ and $i=3$ are the complex conjugates of the ones produced when $h=3$ and $i=7$. Inside the curly brackets, the conjugate of each term is actually produced by a different term. For example, when $h$ and $i$ are reversed, $\left(\Delta q_{h 1}^{\star}\right)^{2} \Delta q_{i 1} \Delta q_{p 1}$ becomes $\left(\Delta q_{i 1}^{*}\right)^{2} \Delta q_{h 1} \Delta q_{p 1}$, which is the 
conjugate of $\Delta q_{h 1}^{*}\left(\Delta q_{i 1}\right)^{2} \Delta q_{p 1}^{*}$. Similar rules apply to the other terms.

As in (3.101), the sum of a term and its conjugate is equal to twice the real part of the term. In (3.122), the real part of each term can be expressed using the sines and cosines of the angles $\Delta \psi_{\mathrm{hl}}, \Delta \psi_{\mathrm{i} 1}, \Delta \psi_{\mathrm{p} 1}$, and $\Delta \Psi_{\mathrm{r} 1}$. When the expected value is taken, the sines vanish as before. The following expressions are needed to obtain the expected value of $\left\langle P_{\Omega}\left(\theta_{1}\right)\right\rangle^{2}$.

$$
\begin{aligned}
E\left[\Delta q_{h 1}^{*} \Delta q_{i 1}+\Delta q_{i 1}^{*} \Delta q_{h 1}\right] & =2 E\left[\operatorname{Re}\left(\Delta q_{h 1}^{*} \Delta q_{i 1}\right\rangle\right] \\
& =2 E\left[\cos \left(\Delta \psi_{i 1}-\Delta \Psi_{h 1}\right)\right] \\
& =2 E\left[\cos \Delta \Psi_{i 1}\right] E\left[\cos \Delta \Psi_{h 1}\right] \\
& =2\left(E\left[\cos \Delta \Psi_{n m}\right]\right)^{2}
\end{aligned}
$$

The terms $\Delta q_{h 1}^{\star} \Delta q_{p 1}$ and $\Delta q_{i 1} \Delta q_{p 1}^{*}$ have the same form as $\Delta q_{h 1}^{*} \Delta q_{i 1}$

$$
\begin{aligned}
& E\left[\Delta q_{h 1}^{*} \Delta q_{p 1}+\Delta q_{p 1}^{*} \Delta q_{h 1}\right]=2\left(E\left[\cos \Delta \psi_{n m}\right]\right)^{2} \\
& E\left[\Delta q_{i 1} \Delta q_{p 1}^{*}+\Delta q_{p 1} \Delta q_{i 1}^{*}\right]=2\left(E\left[\cos \Delta \psi_{n m}\right]\right)^{2}
\end{aligned}
$$




$$
\begin{aligned}
E\left[\left(\Delta q_{h 1}^{*} \Delta q_{i 1}\right)^{2}+\left(\Delta q_{i 1}^{*} \Delta q_{h 1}\right)^{2}\right] \\
=2 E\left[\operatorname{Re}\left(\left(\Delta q_{h 1}^{*} \Delta q_{i 1}\right)^{2}\right)\right] \\
=2 E\left[\cos \left(2 \Delta \psi_{i 1}-2 \Delta \psi_{h 1}\right)\right] \\
=2 E\left[\cos 2 \Delta \psi_{i 1}\right] E\left[\cos 2 \Delta \psi_{h 1}\right] \\
=2\left(E\left[\cos 2 \Delta \psi_{n m}\right]\right)^{2}
\end{aligned}
$$

$$
\begin{aligned}
& E\left[\left(\Delta q_{h 1}^{*}\right)^{2} \Delta q_{i 1} \Delta q_{p 1}+\Delta q_{i 1}^{*}\left(\Delta q_{h 1}\right)^{2} \Delta q_{p 1}^{*}\right] \\
& =2 E\left[R e\left(\left(\Delta q_{h 1}^{\star}\right)^{2} \Delta q_{i 1} \Delta q_{p 1}\right\}\right] \\
& =2 E\left[\cos \left(\Delta \Psi_{i 1}+\Delta \Psi_{p 1}-2 \Delta \psi_{h 1}\right)\right] \\
& =2 \mathrm{E}\left[\cos \Delta \psi_{\mathrm{i} 1}\right] \mathrm{E}\left[\cos \Delta \psi_{\mathrm{p} 1}\right] \mathrm{E}\left[\cos 2 \Delta \psi_{\mathrm{h} 1}\right] \\
& =2\left(E\left[\cos \Delta \psi_{\mathrm{nm}}\right]\right)^{2} \mathrm{E}\left[\cos 2 \Delta \psi_{\mathrm{nm}}\right]
\end{aligned}
$$

The term $\Delta q_{h l}^{\star}\left(\Delta q_{i 1}\right)^{2} \Delta q_{p l}^{\star}$ plus its conjugate has the same form as (3.127).

$$
\begin{aligned}
E\left[\Delta q_{h 1}^{\star}\left(\Delta q_{i 1}\right)^{2} \Delta q_{p 1}^{\star}+\left(\Delta q_{i 1}^{*}\right)^{2} \Delta q_{h 1} \Delta q_{p 1}\right] \\
=2\left(E\left[\cos \Delta \psi_{n m}\right]\right)^{2} E\left[\cos 2 \Delta \psi_{n m}\right]
\end{aligned}
$$

$$
\begin{aligned}
& E\left[\Delta q_{h 1}^{*} \Delta q_{i 1} \Delta q_{p 1}^{*} \Delta q_{r 1}+\Delta q_{h 1} \Delta q_{i 1}^{*} \Delta q_{p 1} \Delta q_{r 1}^{*}\right] \\
&=2 E\left[\operatorname{Re}\left(\Delta q_{h 1}^{*} \Delta q_{i 1} \Delta q_{p 1}^{*} \Delta q_{r 1}\right)\right] \\
&=2 E\left[\cos \left(\Delta \psi_{i 1}-\Delta \psi_{h 1}+\Delta \psi_{r 1}-\Delta \psi_{p 1}\right)\right] \\
&=2 E\left[\cos \Delta \Psi_{i 1}\right] E\left[\cos \Delta \psi_{h 1}\right] E\left[\cos \Delta \psi_{r 1}\right] E\left[\cos \Delta \psi_{p 1}\right] \\
&=2\left(E\left[\cos \Delta \Psi_{n m}\right]\right)^{4}
\end{aligned}
$$

Applying these expressions to (3.122), the mean square value of $P_{\Omega}\left(\theta_{1}\right)$ is as follows. 


$$
\begin{aligned}
& E\left[\left(P_{\Omega}\left(\theta_{1}\right)\right)^{2}\right]=N^{2} \Lambda^{2}+2 N^{2}(N-1) \wedge \zeta\left(E\left[\cos \Delta \psi_{n m}\right]\right)^{2} \\
& +N(N-1) \zeta^{2}\left(E\left[\cos \Delta \psi_{n m}\right]\right)^{2}+N(N-1) \zeta^{2} \\
& +2 N(N-1)(N-2) \zeta^{2}\left\{\left(E\left[\cos \Delta \psi_{n m}\right]\right)^{2} E\left[\cos 2 \Delta \psi_{n m}\right]\right. \\
& \left.+\left(E\left[\cos \Delta \psi_{n m}\right]\right)^{2}\right\} \\
& +N(N-1)(N-2)(N-3) \zeta^{2}\left(E\left[\cos \Delta \psi_{n m}\right]\right)^{4}
\end{aligned}
$$

The calculation of the variance of $P_{\Omega}\left(\theta_{1}\right)$ also requires the square of the mean. From $(3.111),\left(E\left[P_{\Omega}\left(\theta_{1}\right)\right]\right)^{2}$ is as follows.

$$
\begin{aligned}
\left\langle E\left[P_{\Omega}\left(\theta_{1}\right)\right]\right\}^{2} & =\left\{N \Lambda+N(N-1) \zeta\left(E\left[\cos \Delta \psi_{n m}\right]\right)^{2}\right\}^{2} \\
& =N^{2} \Lambda^{2}+2 N^{2}(N-1) \wedge \zeta\left(E\left[\cos \Delta \psi_{n m}\right]\right)^{2} \\
& +N^{2}(N-1)^{2} \zeta^{2}\left(E\left[\cos \Delta \psi_{n m}\right]\right)^{4}
\end{aligned}
$$

The variance of $P_{\Omega}\left(\theta_{1}\right)$ is the mean square value minus the square of the mean [97].

$$
V\left[P_{\Omega}\left(\theta_{1}\right)\right]=E\left[\left(P_{\Omega}\left(\theta_{1}\right)\right\rangle^{2}\right]-\left\langle E\left[P_{\Omega}\left(\theta_{1}\right)\right]\right\rangle^{2}
$$

Substituting (3.130) and (3.131) into (3.132), the variance of $P_{\Omega}\left(\theta_{1}\right)$ is as follows. 


$$
\begin{aligned}
\mathrm{V}\left[\mathrm{P}_{\Omega}\left(\theta_{1}\right)\right] & =\mathrm{N}(\mathrm{N}-1) \zeta^{2}\left[2(3-2 \mathrm{~N})\left(\mathrm{E}\left[\cos \Delta \Psi_{\mathrm{nm}}\right]\right)^{4}\right. \\
& +2(\mathrm{~N}-2)\left\{\mathrm{E}\left[\cos 2 \Delta \Psi_{\mathrm{nm}}\right]+1\right\}\left(E\left[\cos \Delta \Psi_{\mathrm{nm}}\right]\right)^{2} \\
& \left.+\left(E\left[\cos \Delta \Psi_{\mathrm{nm}}\right]\right)^{2}+1\right]
\end{aligned}
$$

(3.133) can be checked (partially) by setting $\sigma_{p}^{z}$ to zero, in which case $E\left[\cos \Delta \psi_{n m}\right]=E\left[\cos 2 \Delta \psi_{n m}\right]=1$ and $v\left[P_{\Omega}\left(\theta_{1}\right)\right]$ evaluates to zero. This confirms that the variance of the peak height (or its inverse) due to sensor positioning is zero when there are no errors present.

\subsection{The Variance of the spectrum Value at the True Bearing}

The variances of $P_{b f}\left(\theta_{1}\right), P_{m e}^{-1}\left(\theta_{1}\right)$, and $P_{m u}^{-1}\left(\theta_{1}\right)$ can be can be expressed in terms of $V\left[P_{\Omega}\left(\theta_{1}\right)\right]$ in much the same way that the means were related to $E\left[P_{\Omega}\left(\theta_{1}\right)\right]$.

$$
\begin{array}{ll}
\mathrm{V}\left[P_{\mathrm{bf}}\left(\theta_{1}\right)\right]=\mathrm{V}\left[\mathrm{P}_{\Omega}\left(\theta_{1}\right)\right], & \zeta=\zeta_{\mathrm{bf}} \\
\mathrm{V}\left[\mathrm{P}_{\mathrm{me}}^{-1}\left(\theta_{1}\right)\right]=\mathrm{V}\left[P_{\Omega}\left(\theta_{1}\right)\right], & \zeta=\zeta_{\mathrm{me}} \\
\mathrm{V}\left[\mathrm{P}_{\mathrm{mu}}^{-1}\left(\theta_{1}\right)\right]=\mathrm{V}\left[\mathrm{P}_{\Omega}\left(\theta_{1}\right)\right], & \zeta=\zeta_{\mathrm{mu}}
\end{array}
$$


An expression for $V\left[P_{\Omega}\left(\theta_{1}\right)\right]$ in terms of $\zeta$ is given in (3.133) and $\zeta_{b f}, \zeta_{m e}$, and $\zeta_{m u}$ are defined by (3.71), (3.79), and (3.85). The variances of $P_{b f}\left(\theta_{1}\right), P_{m}^{-1}\left(\theta_{1}\right)$, and $P_{\mathrm{mu}}^{-\frac{1}{(}\left(\theta_{1}\right)}$ are therefore as follows.

$$
\begin{aligned}
& \mathrm{V}\left[\mathrm{P}_{\mathrm{bf}}\left(\theta_{1}\right)\right]=N(N-1) A_{1}^{4}\left[2(3-2 N)\left(E\left[\cos \Delta \psi_{\mathrm{nm}}\right]\right)^{4}\right. \\
& +2(N-2)\left\{E\left[\cos 2 \Delta \psi_{n m}\right]+1\right\}\left(E\left[\cos \Delta \psi_{n m}\right]\right)^{2} \\
& \left.+\left(E\left[\cos \Delta \psi_{\mathrm{nm}}\right]\right)^{2}+1\right] \\
& V\left[P_{m e}^{-1}\left(\theta_{1}\right)\right]=\frac{N(N-1) A_{1}^{4}}{\sigma_{W}^{4}\left(\sigma_{W}^{2}+N A_{1}^{2}\right)^{2}}\left[2(3-2 N)\left(E\left[\cos \Delta \Psi_{n m}\right]\right)^{4}\right. \\
& +2(N-2)\left\{E\left[\cos 2 \Delta \psi_{n m}\right]+1\right\}\left(E\left[\cos \Delta \psi_{n m}\right]\right)^{2} \\
& \left.+\left(E\left[\cos \Delta \psi_{n m}\right]\right)^{2}+1\right] \\
& V\left[P_{m}^{-1}\left(\theta_{1}\right)\right]=\frac{1}{N}(N-1)\left[2(3-2 N)\left(E\left[\cos \Delta \psi_{n m}\right]\right)^{4}\right. \\
& +2(N-2)\left\{E\left[\cos 2 \Delta \psi_{n m}\right]+1\right\}\left(E\left[\cos \Delta \psi_{n m}\right]\right)^{2} \\
& \left.+\left(E\left[\cos \Delta \Psi_{n m}\right]\right)^{2}+1\right]
\end{aligned}
$$


Figure 3.25 compares the simulation results for the variances of $P_{b f}\left(\theta_{1}\right), P_{m e}^{-1}\left(\theta_{1}\right)$, and $P_{m}^{-1}\left(\theta_{1}\right)$ with the curves predicted using $(3.137),(3.138)$, and (3.139). In all three cases, the variance increases with $\sigma_{\mathrm{p}}^{2}$.

\subsection{The Mean and variance of the Peak Height}

Although the theoretical work discussed in this chapter does not predict the means and variances of $\mathrm{P}_{\mathrm{bf}}\left(\theta_{1}+\Delta \theta_{1}\right)$, $P_{\mathrm{me}}^{-1}\left(\theta_{1}+\Delta \theta_{1}\right)$, and $P_{\mathrm{mu}}^{-1}\left(\theta_{1}+\Delta \theta_{1}\right)$, it is possible to produce bounds for these means and variances using the expressions for the means and variances of $P_{b f}\left(\theta_{1}\right)$, $\mathrm{P}_{\mathrm{me}}^{-1}\left(\theta_{1}\right)$, and $\mathrm{P}_{\mathrm{mu}}^{-1}\left(\theta_{1}\right)$ derived earlier.

Since the peak is, by definition, the highest point in the spectrum, the peak height $P\left(\theta_{1}+\Delta \theta_{1}\right)$ must be greater than $P\left(\theta_{1}\right)$ whenever $\Delta \theta_{1} \neq 0$. When $\Delta \theta_{1}=0, \theta_{1}+\Delta \theta_{1}=\theta_{1}$ and $P\left(\theta_{1}+\Delta \theta_{1}\right)$ must be equal to $P\left(\theta_{1}\right)$. It follows that in the general case $P\left(\theta_{1}+\Delta \theta_{1}\right) \geqslant P\left(\theta_{1}\right)$, which in turn impplies that $\mathrm{P}^{-1}\left(\theta_{1}+\Delta \theta_{1}\right) \leqslant \mathrm{P}^{-1}\left(\theta_{1}\right)$. These observations lead to the following inequalities for the peak heights (or their inverses). 
(a) $\quad v\left[P_{b f}\left(\theta_{1}\right)\right]$

6000

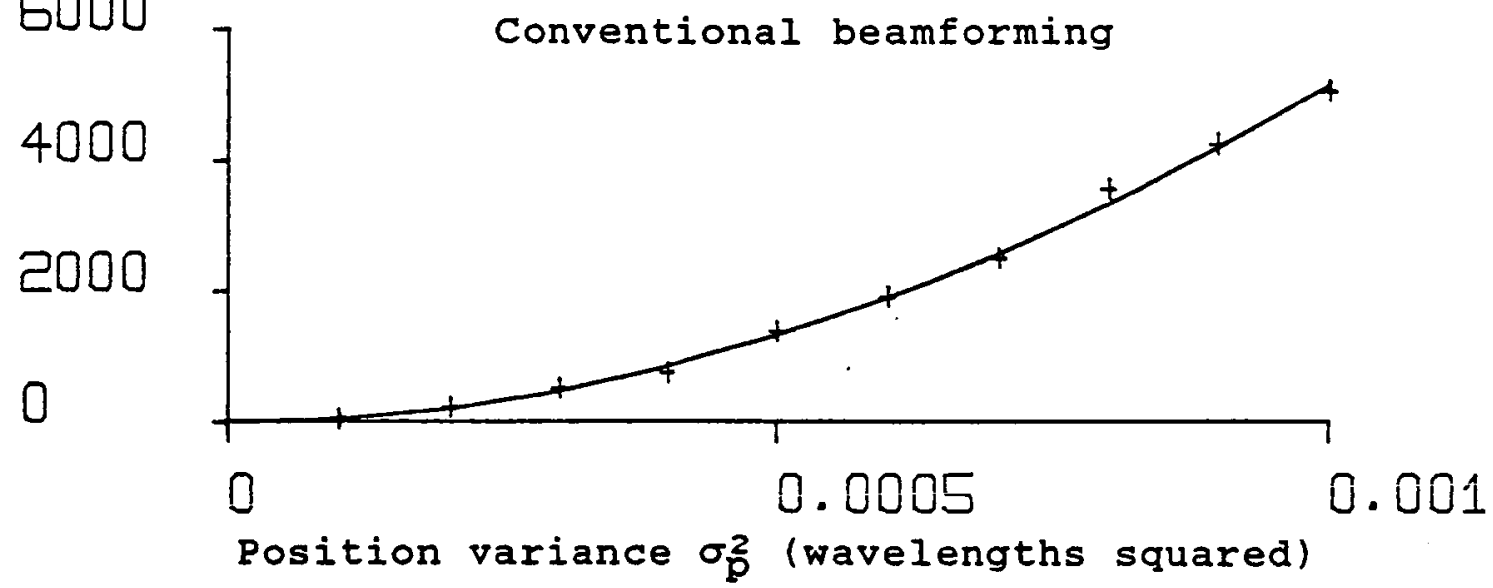

(b) $\quad v\left[P_{m}^{-1}\left(\theta_{1}\right)\right]$
0.015
0.015
0.005
0

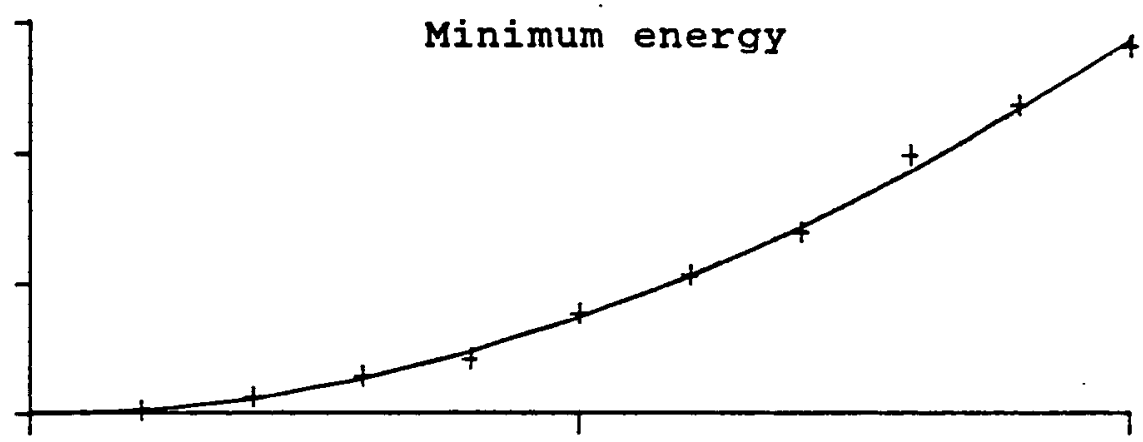

0

Position variance $\sigma_{p}^{2}$ (wavelengths squared)

(c) $\quad \mathrm{v}\left[\mathrm{P}_{\mathrm{m}}^{-\frac{1}{\mathrm{u}}}\left(\theta_{1}\right)\right]$
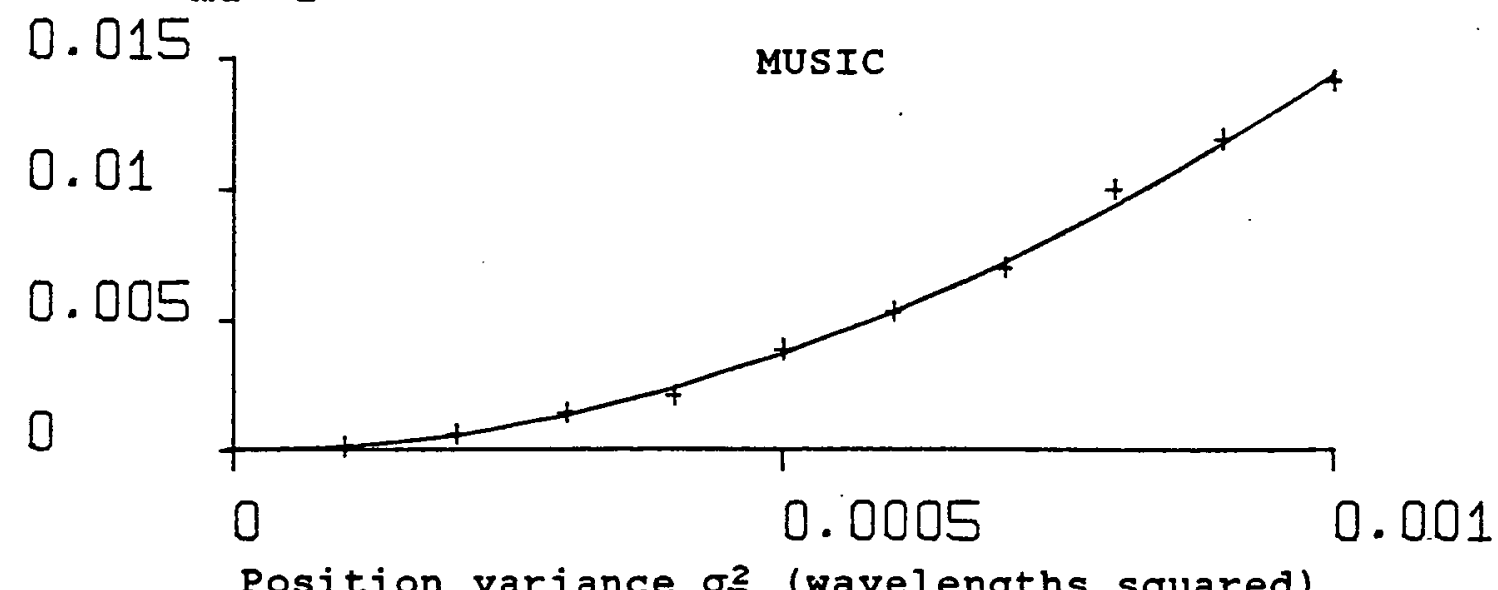

Figure 3.25 Results for variance of spectrum height at true bearing 


$$
\begin{aligned}
& P_{b f}\left(\theta_{1}+\Delta \theta_{1}\right) \geqslant P_{b f}\left(\theta_{1}\right) \\
& \operatorname{P}_{\operatorname{me}}^{-1}\left(\theta_{1}+\Delta \theta_{1}\right) \leqslant P_{m e}^{-1}\left(\theta_{1}\right) \\
& P^{-1} \underline{m}\left(\theta_{1}+\Delta \theta_{1}\right) \leqslant P_{m}^{-1}\left(\theta_{1}\right)
\end{aligned}
$$

Taking the expected value of both sides in (3.140), (3.141), and (3.142), the following expressions for the mean of the peak height are obtained.

$$
\begin{aligned}
& E\left[P_{b f}\left(\theta_{1}+\Delta \theta_{1}\right)\right] \geqslant E\left[P_{b f}\left(\theta_{1}\right)\right] \\
& E\left[P_{m}^{-1}\left(\theta_{1}+\Delta \theta_{1}\right)\right] \leqslant E\left[P_{m e}^{-1}\left(\theta_{1}\right)\right] \\
& E\left[P_{\overline{m u}}^{-1}\left(\theta_{1}+\Delta \theta_{1}\right)\right] \leqslant E\left[P_{\overline{m u}}^{-1}\left(\theta_{1}\right)\right]
\end{aligned}
$$

As $\sigma_{\mathfrak{p}}^{2}$ increases, $v\left[\Delta \theta_{1}\right]$ increases (as shown earlier) and large values of $\Delta \theta_{1}$ become more common. As a result, the spectrum point represented by $P\left(\theta_{1}\right)$ tends to be further from the peak. Thus the gap between $E\left[P\left(\theta_{1}+\Delta \theta_{1}\right)\right]$ and $E\left[P\left(\theta_{1}\right)\right]$ widens as $\sigma_{p}^{2}$ increases.

These predictions are confirmed by Figure 3.26, which compares the simulation results for $\mathrm{E}\left[\mathrm{P}_{\mathrm{bf}}\left(\theta_{1}+\Delta \theta_{1}\right)\right]$, $E\left[P_{m e}^{-1}\left(\theta_{1}+\Delta \theta_{1}\right)\right]$, and $E\left[P_{\operatorname{mu}}^{-1}\left(\theta_{1}+\Delta \theta_{1}\right)\right]$ with the theoretical curves for $E\left[P_{b f}\left(\theta_{1}\right)\right], E\left[P_{m e}^{-1}\left(\theta_{1}\right)\right]$, and $E\left[P_{\mathfrak{m u}}^{-1}\left(\theta_{1}\right)\right]$. 
(a) $E\left[P_{b f}(\theta)\right]$

3700 Conventional beamforming

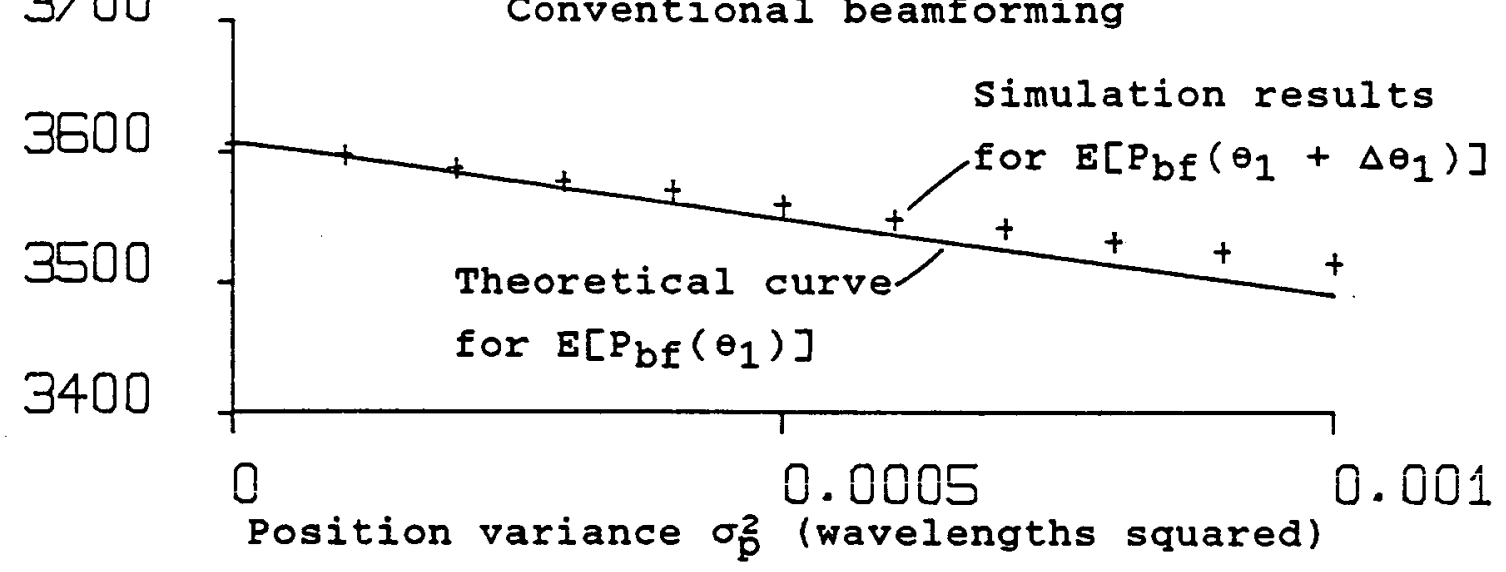

(b)

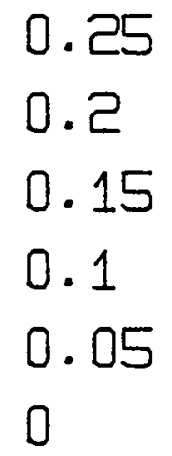

Minimum energy

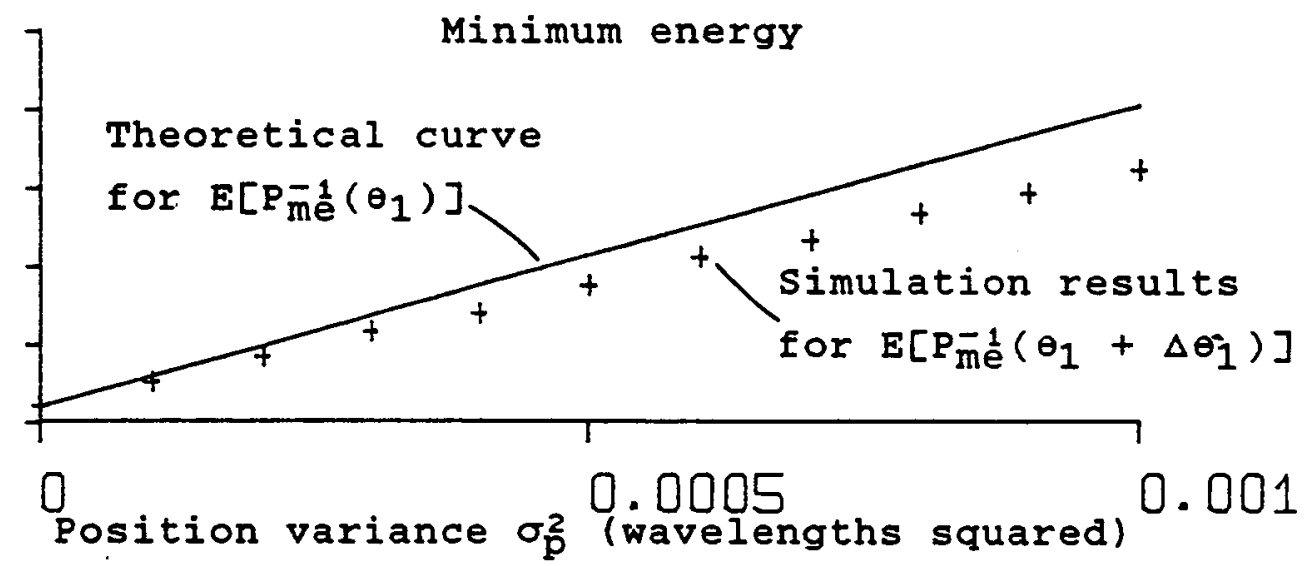

(c) $E\left[P \frac{-1}{m u}(\theta)\right]$

0.25
0.2
0.15
0.1
0.05
0

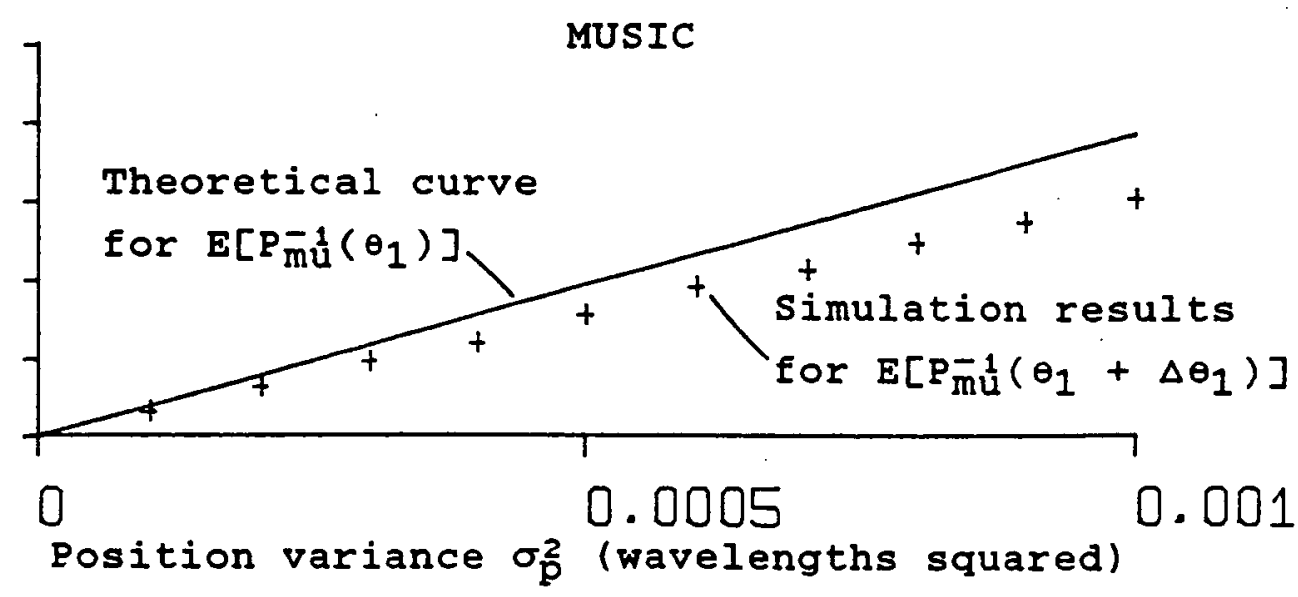

Figure 3.26 Results for mean of peak height 
A similar set of inequalities is obtained for the variances of $P_{b f}\left(\theta_{1}+\Delta \theta_{1}\right), P_{m e}^{-1}\left(\theta_{1}+\Delta \theta_{1}\right)$, and $\mathrm{P}_{\mathrm{mu}}^{-1}\left(\theta_{1}+\Delta \theta_{1}\right)$. Figure 3.27 compares the simulation results for $V\left[P_{b f}\left(\theta_{1}+\Delta \theta_{1}\right)\right], V\left[P_{m e}^{-1}\left(\theta_{1}+\Delta \theta_{1}\right)\right]$, and $V\left[P^{-1} u^{-1}\left(\theta_{1}+\Delta \theta_{1}\right)\right]$ with the theoretical curves for $V\left[P_{b f}\left(\theta_{1}\right)\right], V\left[P_{m e}^{-1}\left(\theta_{1}\right)\right]$, and $V\left[P_{m}^{-1}\left(\theta_{1}\right)\right]$. By inspection, the bounds on the variance of the peak height are as follows.

$$
\begin{aligned}
& \mathrm{V}\left[P_{b f}\left(\theta_{1}+\Delta \theta_{1}\right)\right] \leqslant V\left[P_{b f}\left(\theta_{1}\right)\right] \\
& V\left[P_{m}^{-1}\left(\theta_{1}+\Delta \theta_{1}\right)\right] \leqslant V\left[P_{m e}^{-1}\left(\theta_{1}\right)\right] \\
& V\left[P_{\overline{m u}}^{-1}\left(\theta_{1}+\Delta \theta_{1}\right)\right] \leqslant V\left[P_{m u}^{-1}\left(\theta_{1}\right)\right]
\end{aligned}
$$

Comparing the results for the mean of the peak height with the results for the variance, notice that (3.146), (3.147), and (3:148) all define upper bounds whereas (3.143), (3.144), and (3.145) specify a lower bound for conventional beamforming and upper bounds for the other two techniques. The reason for this is that as $\sigma_{\mathbb{P}}^{2}$ increases, $E\left[P_{b f}\left(\theta_{1}\right)\right]$ decreases and $v\left[P_{b f}\left(\theta_{1}\right)\right]$ increases. Thus an increase in $E\left[P_{b f}\left(\theta_{1}\right)\right]$ is accompanied by a decrease in $E\left[P_{b f}\left(\theta_{1}\right)\right]$. Since $E\left[P_{b f}\left(\theta_{1}+\Delta \theta_{1}\right)\right]$ is greater than $E\left[P_{b f}\left(\theta_{1}\right)\right]$, the corresponding value of $\mathrm{V}\left[\mathrm{P}_{\mathrm{bf}}\left(\theta_{1}+\Delta \theta_{1}\right)\right]$ is less than $\mathrm{V}\left[\mathrm{P}_{\mathrm{bf}}\left(\theta_{1}\right)\right]$. 
(a) $\quad v\left[P_{b f}(\theta)\right]$

6000

4000
2000
0

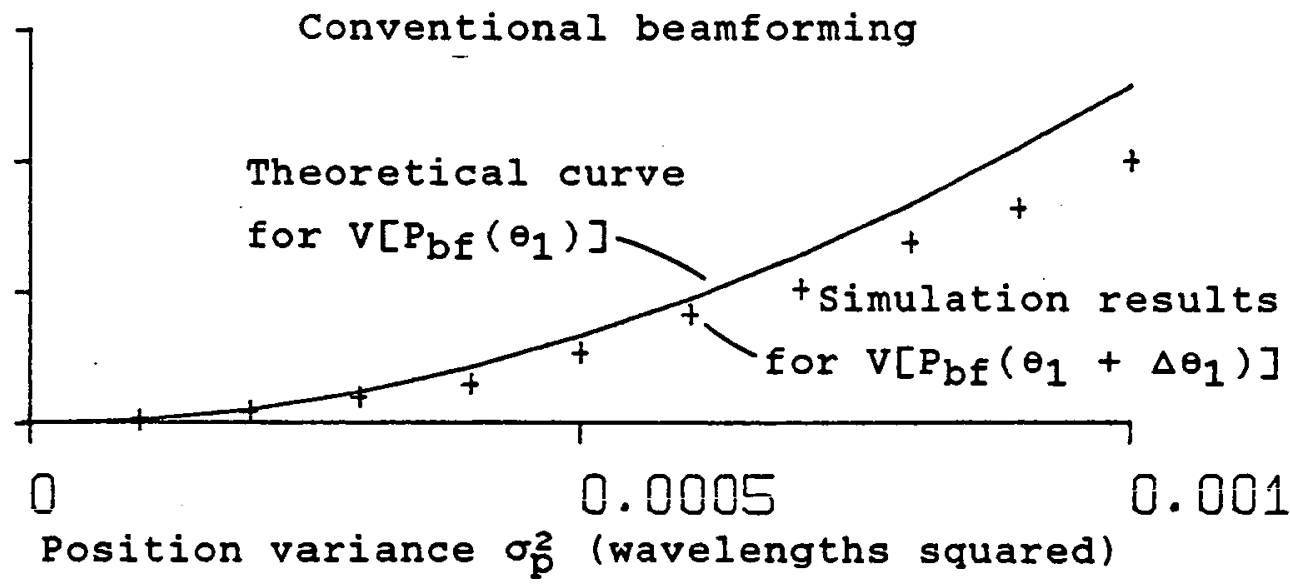

(b)

0.015
0.01
0.005
0

$\left[P_{\operatorname{me}}^{-1}(\theta)\right]$

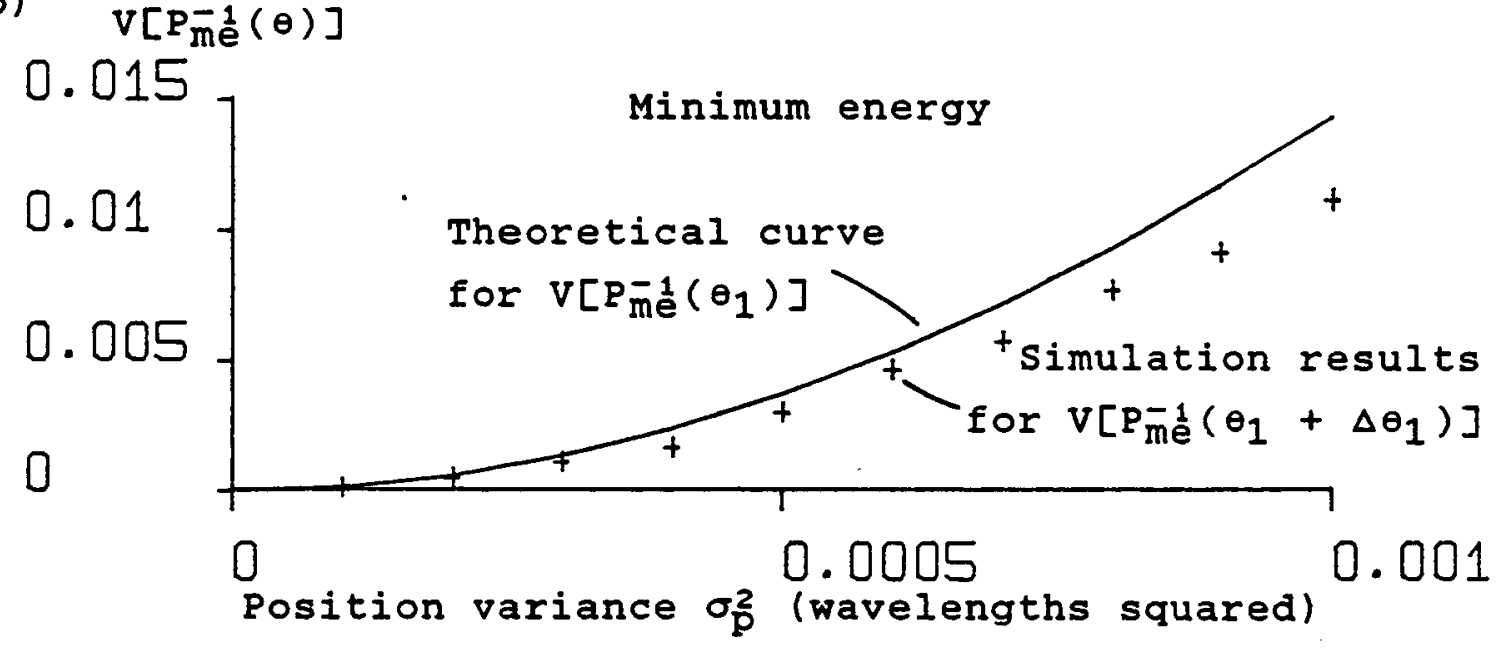

(c)

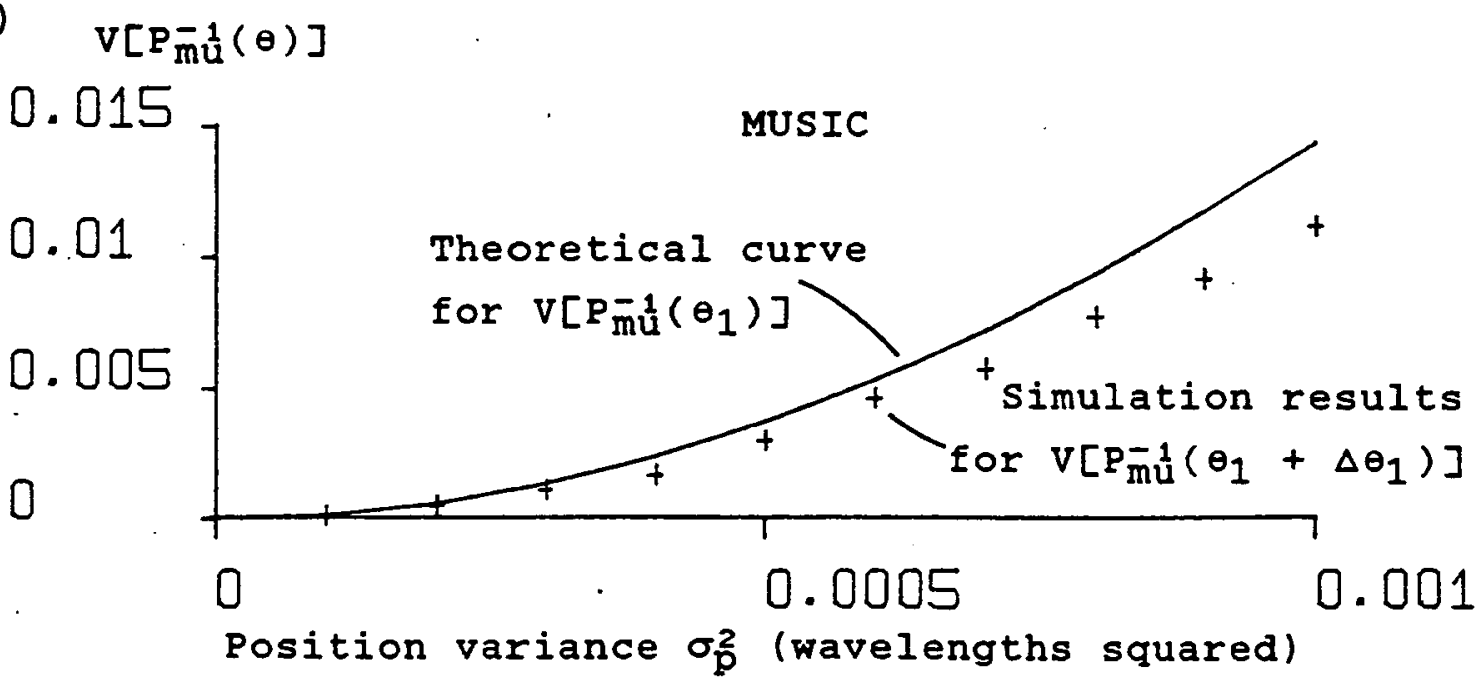

Figure 3.27 Results for variance of peak height 
In the case of minimum energy, however, $\operatorname{E}\left[\mathrm{P}_{\mathrm{me}}^{-1}\left(\theta_{1}\right)\right]$ and $\mathrm{V}\left[\mathrm{P}_{\mathrm{me}}^{-1}\left(\theta_{1}\right)\right]$ both increase as $\sigma_{\mathrm{p}}^{2}$ is increased. Since $E\left[P_{m e}^{-1}\left(\theta_{1}+\Delta \theta_{1}\right)\right]$ is less than $E\left[P_{m e}^{-1}\left(\theta_{1}\right)\right]$, it follows that $\mathrm{V}\left[\mathrm{P}_{\mathrm{me}}^{-1}\left(\theta_{1}+\Delta \theta_{1}\right)\right]$ is less than $\mathrm{V}\left[\mathrm{P}_{\mathrm{me}}^{-1}\left(\theta_{1}\right)\right]$. The same argument applies to MUSIC.

\subsection{8 summary}

This chapter has considered the effects of sensor positioning errors on the bearing estimation process. The first step was to define notation for the errors in the sensor positions and the resulting errors in the phase measurements obtained from the array.

The bulk of the chapter was devoted to two pieces of theoretical work, the first of which derived expressions for the mean and variance of the error in the estimated bearing of a signal in terms of the variance of the sensor positions. This material was based on a geometric view of bearing estimation which was explained through a series of graphical constructions.

The second piece of work addressed the behaviour of the bearing spectrum at the true bearing of the signal. This material was mainly algebraic in nature and showed how the 
sensor positioning errors coupled through the conventional beamforming, minimum energy, and MUSIC spectral estimators introduced in Chapter 2 .

Computer simulations were used to confirm the theoretical results and also to achieve some insight into effects which were not covered by the theoretical work. In particular, it was shown that the theoretical expressions describing the spectrum at the true bearing of the signal could be used to construct bounds on the mean and variance of the peak height. 


\section{CHAPTER 4 AN ARRAY CALIBRATION ALGORITHM}

\subsection{Introduction}

This chapter develops an array calibration algorithm to compensate for sensor positioning errors in direction finding arrays, the aim being to provide a solution to the problems identified in the last chapter. Like the material presented in Chapter 3 , the calibration algorithm is an original piece of research.

As explained earlier, there are two distinct sets of sensor positions, referred to as the intended positions and the true ones. The intended positions model the ideal array geometry, while the (unknown) true positions incorporate the sensor positioning errors and are the result of one particular attempt at setting the array up.

\footnotetext{
Although the snapshots obtained from the array are influenced by the true sensor positions, the bearing estimation algorithm used to analyze the snapshots normally operates on signal vectors derived from the intended positions. This inconsistency lowers the quality of the bearing spectrum as demonstrated in Chapter 3 .
} 
If the true sensor positions could be discovered, the bearing estimation algorithm could be given signal vectors corresponding to these positions rather than the intended ones and the inconsistency would be removed.

The calibration algorithm described in this chapter attempts to deduce the true sensor positions by analyzing snapshots obtained while signals with known bearings are being received. If the errors in the sensor positions are small, the intended positions provide good initial estimates of the true ones and the accuracy to which the true positions can be estimated is limited only by the quality of the covariance matrix, which depends on factors such as the signal to noise ratio and the number of snapshots processed.

Once the estimated sensor positions have been obtained, they are used in place of the intended ones when performing bearing estimation on unknown signals. Provided that the estimated positions are closer to the true positions than the intended ones were, some improvement can be expected in the performance of the bearing estimation algorithm.

The general layout of this chapter is similar to that of Chapter 3. The problem is approached initially from an intuitive standpoint using a series of graphical 
constructions which in turn suggest a more mathematical treatment. Computer simulations are used to investigate the performance of the calibration algorithm.

\subsection{Array Calibration and Bearing Estimation}

Since array calibration and bearing estimation are essentially inverse problems, it is instructive to consider the similarities and differences between them. In both cases, the presence of signal $m$ reveals the component $\epsilon_{\mathrm{nm}}$ of the true position ${\underset{\sim}{n}}_{\mathrm{n}}$ of each sensor in the direction of the vector $\mathbf{z}_{\mathrm{m}}$.

In bearing estimation, $z_{m}$ is initially unknown and it is necessary to explore a range of bearings as shown in Figure $4.1(a)$. In general, it is only possible to reconcile all of the distances $\epsilon_{\mathrm{nm}}$ with the assumed sensor positions when the axis of transmission of signal $m$ has been identified correctly as illustrated in Figure $4.1(b)$.

The exception to this rule arises when ambiguities are present as in the linear array example described in Chapter 2. In this case, the observed distances $\epsilon_{\mathrm{nm}}$ can be accounted for by bearings other than the true one. 
(a)

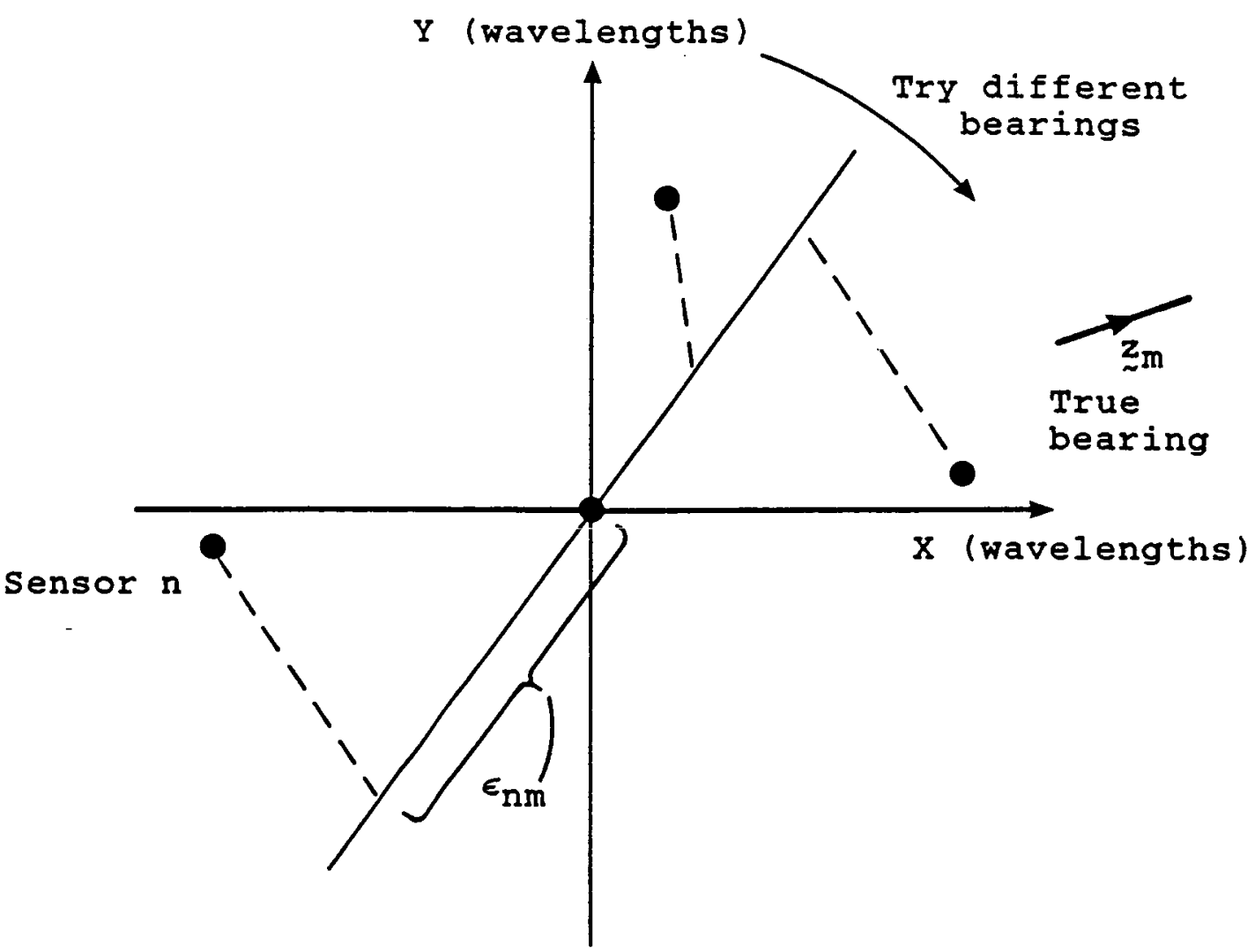

(b)

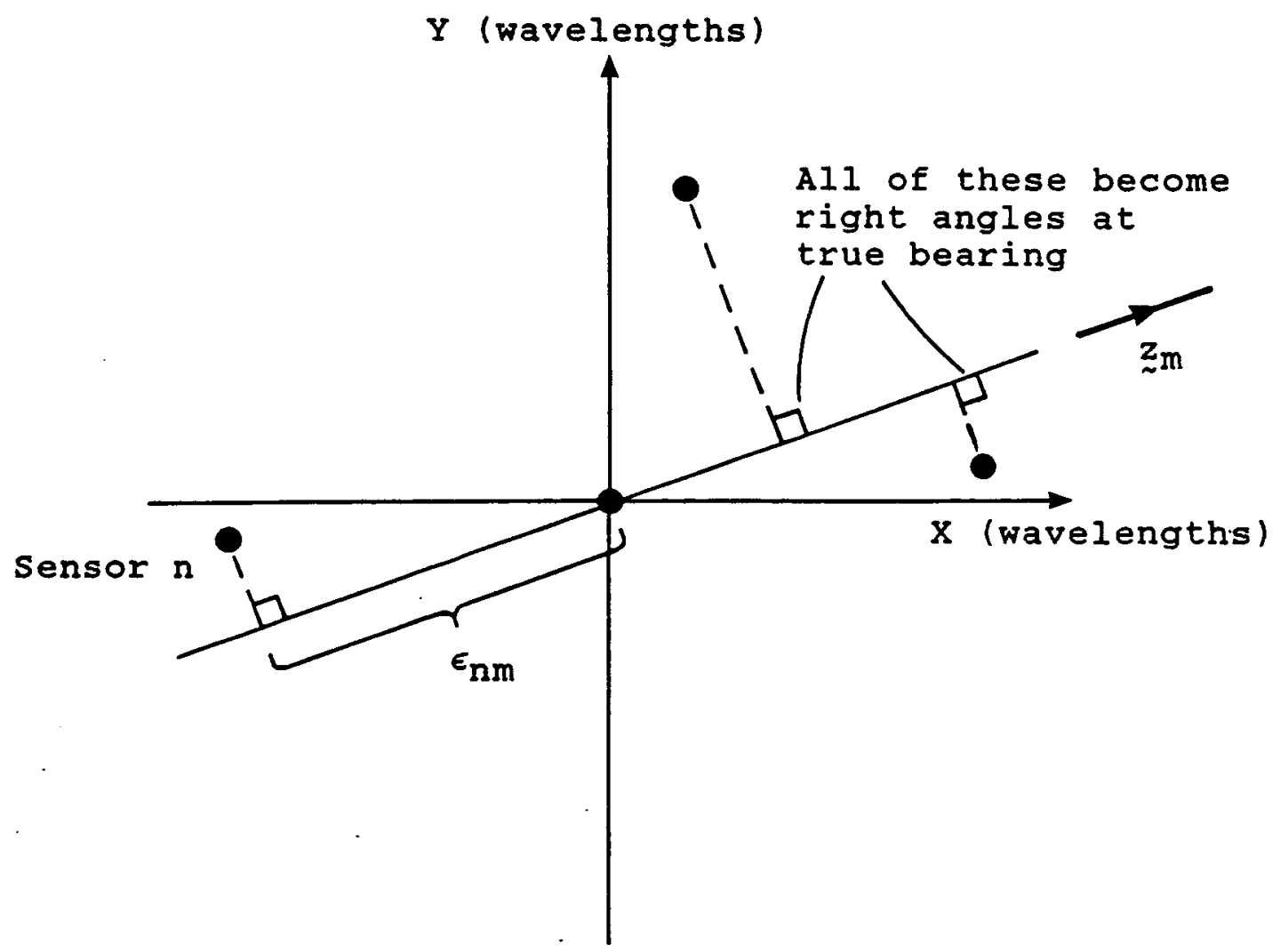

Figure 4.1 Bearing estimation 
In array calibration, the signal bearings are assumed to be known already. Figure $\mathbf{4 . 2}$ (a) shows an array receiving a single transmission from the direction $z_{1}$. The vector $z_{1}$ and the distance $\epsilon_{n 1}$ provide information about the position of sensor $n$ since the sensor must lie on the line perpendicular to $z_{1}$ passing through the point $\epsilon_{\mathrm{n}_{1} z_{1}}$ as shown by the dashed line in the diagram.

In principle, there is an ambiguity in the value of $\epsilon_{\mathrm{nm}}$ since the measurement is made indirectly through the phasor $q_{n m}$, which contains an unknown number of $2 \pi$ cycles. However, the distance $\hat{\epsilon}_{\mathrm{nm}}$ derived (unambiguously) from the intended sensor positions is likely to be very close to $\epsilon_{\mathrm{nm}}$ and the matter can therefore be resolved by identifying the possible values for $\epsilon_{\mathrm{nm}}$ based on $q_{\mathrm{nm}}$ then choosing the one closest to $\hat{\epsilon}_{\mathrm{nm}}$.

The construction shown in Figure $4.2(a)$ only reveals the components of the sensor positions in one direction. To determine the positions completely, a signal from a second direction $z_{2}$ must be processed as shown in Figure 4.2(b). This provides the additional information that sensor $n$ lies on the line perpendicular to $z_{2}$ passing through the point $\epsilon_{\mathrm{n} 2 \underset{\sim}{z} 2}$.

Combining the two constraints, it follows that the true position of sensor $\mathrm{n}$ is at the intersection of the two 
(a)

Y (wavelengths)

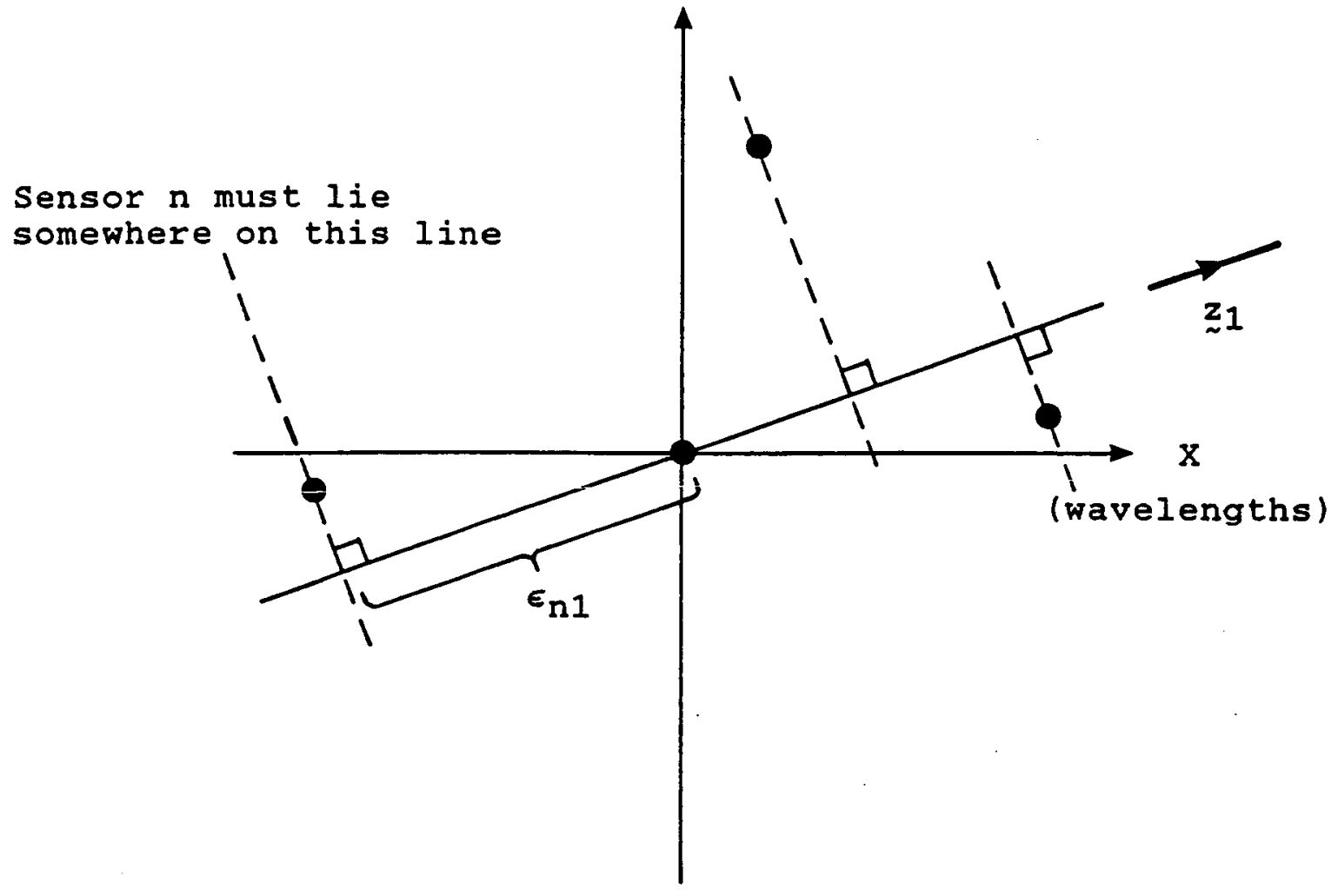

(b)

Y (wavelengths)

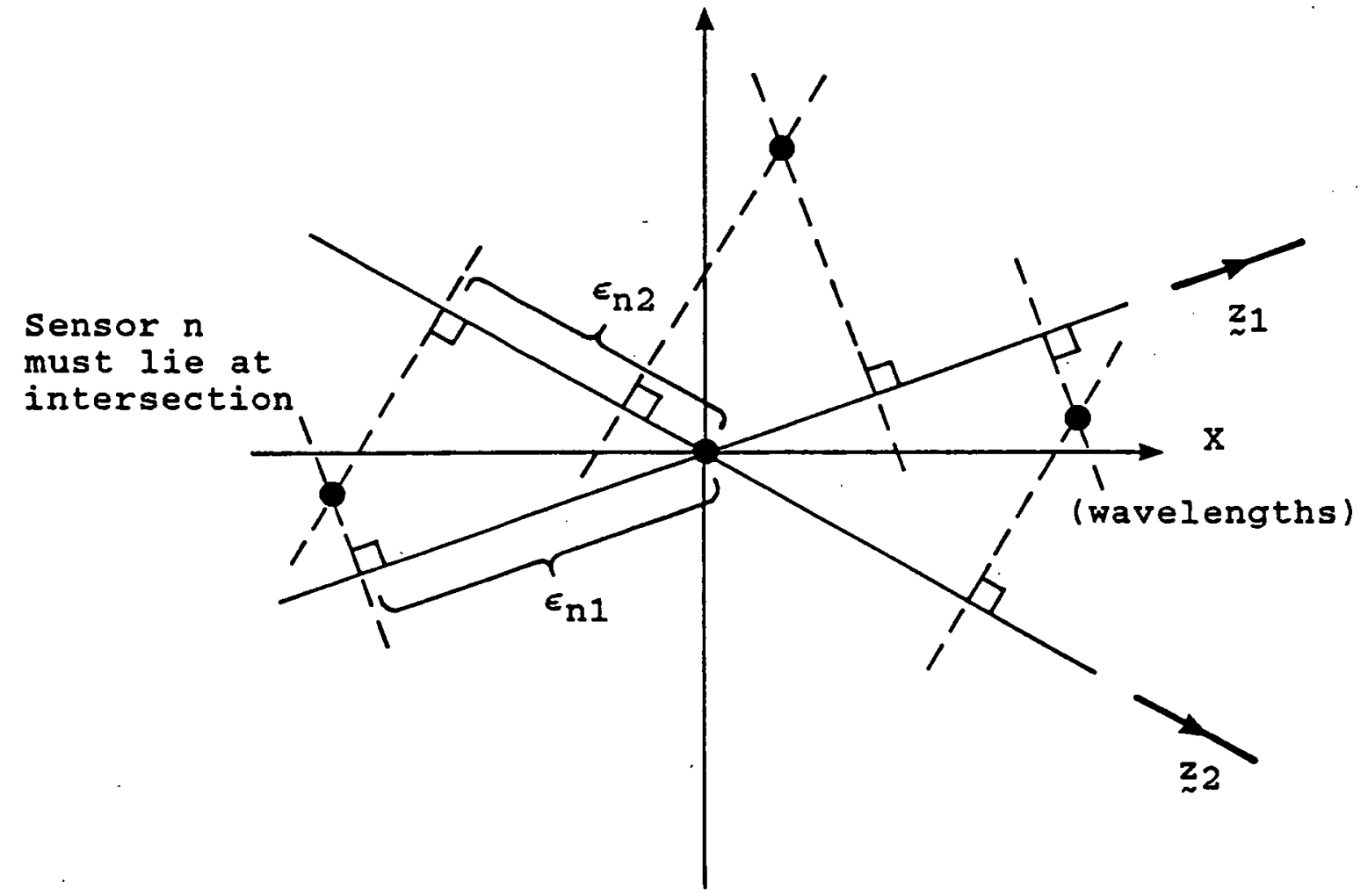

Figure 4.2 Array calibration 
dashed lines as illustrated. The process is essentially a novel application of triangulation. In common with other forms of triangulation, processing of more than two directions provides increased confidence about the point of intersection. The next section describes a particular advantage of using three or more signals for array calibration.

\subsection{Sources of Error in Array Calibration}

Although the calibration procedure is based on the assumption that the signal bearings are known accurately, it is important to understand how the performance of the method degrades when this assumption is false. Any errors in the directions of the vectors $\mathbf{z}_{n}$ cause the sensor positions to be estimated incorrectly.

By analogy with the approach used in Chapter 3 for bearing estimation, it is possible to identify true and intended bearings for the calibration signals. Snapshots from the array are determined by the true bearings but are analyzed using the intended ones.

Figure $4.3(a)$ shows the effect of bearing errors if there are only two calibration signals present. When 


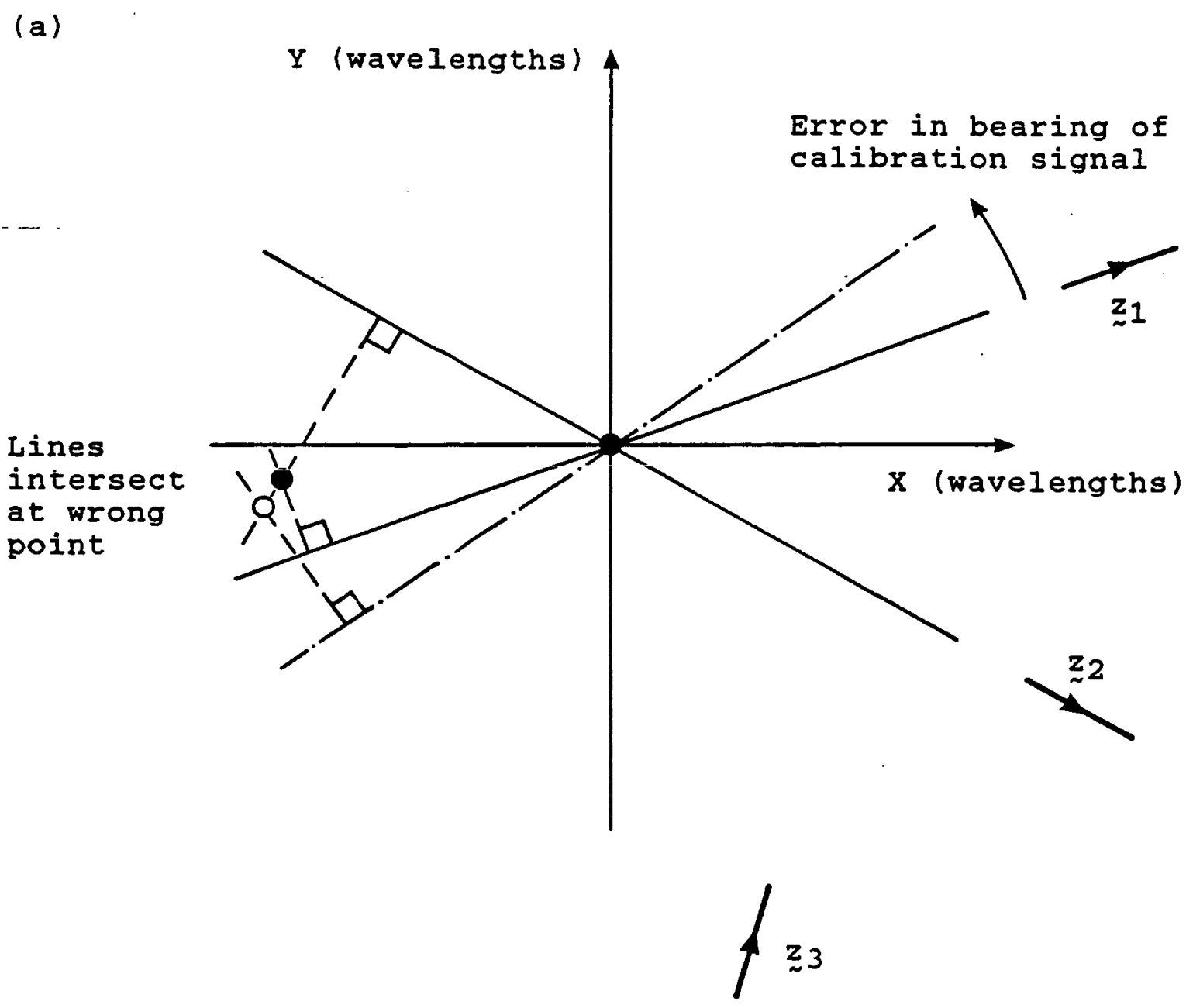

(b)

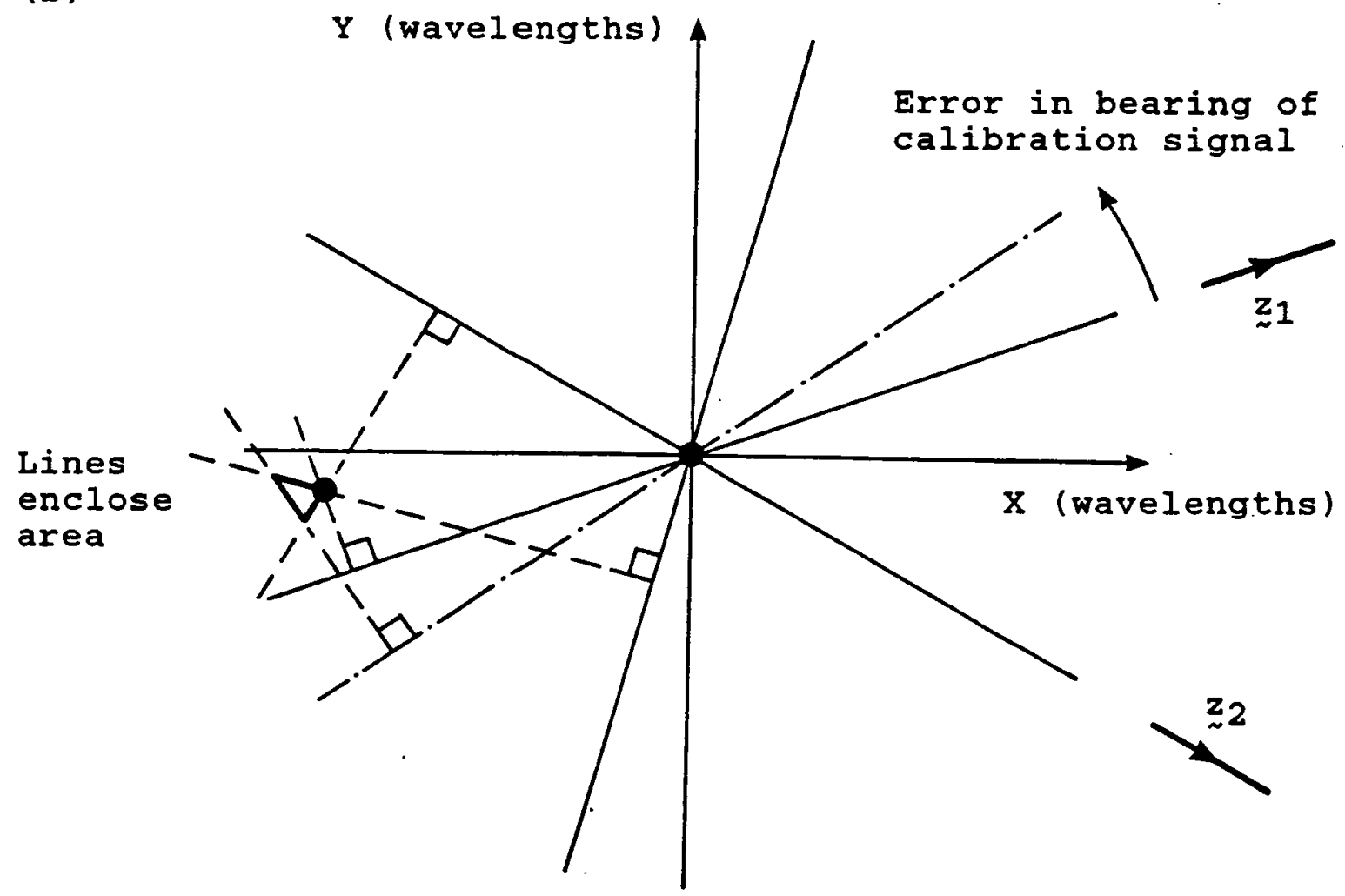

Figure 4.3 Errors in bearings of calibration signals 
triangulation is performed to locate sensor $n$, the dashed lines intersect at the wrong point. There is no way to detect this error.

Figure $4.3(\mathrm{~b})$ demonstrates the advantage of using more than two signals. When bearing errors are present, the dashed lines generally enclose an area rather than intersecting at a point and this provides a warning that the triangulation has failed.

Even if the vectors ${\underset{\sim}{m}}_{m}$ are known accurately, errors can arise in the measurement of the distances $\epsilon_{n m}$, which are obtained from the off-diagonal elements of the covariance matrix $\underset{\sim}{R}$. As explained in Chapter 2, the effect of received noise is confined to the leading diagonal of $\underset{\sim}{R}$ when the expected value is calculated. However, for practical measurements based on a finite number of snapshots, some corruption of the off-diagonal elements is inevitable. These errors are most pronounced when the signal to noise ratio is low and/or a small number of snapshots are used.

Errors in the distances $\epsilon_{\mathrm{nm}}$ are similar in effect to errors in the vectors $\mathbf{z}_{m}$, causing the triangulation to be performed using inappropriate lines. As before, the problem can usually be detected if more than two calibration signals are present. Indeed, if the 
triangulation does produce an area rather than a point, the errors could be in the vectors ${\underset{\sim}{m}}_{\mathrm{m}}$, the distances $\epsilon_{\mathrm{nm}}$. or both.

Throughout this discussion, it is acknowledged that the lines used for triangulation are unlikely to intersect perfectly at one point even under favourable conditions. Thus references to the Iines crossing at a point also include the case of the lines enclosing a very small area.

To summarize, the performance of the calibration procedure depends on factors such as the accuracy to which the bearings of the signals are known, the signal to noise ratio, and the number of snapshots used to form the covariance matrix. Problems in any of these areas produce errors which affect the triangulation process in similar ways and this is demonstrated using computer simulations at the end of the chapter.

\subsection{An Iterative Approach to Array Calibration}

Having established the basic idea of using triangulation to discover the true sensor positions, the next step is to develop a precise mathematical interpretation of the process. The observations made in the previous section 
regarding the effects of errors have some influence on the choice of algorithm.

Since triangulation involves calculating the point of intersection of a number of lines, the problem could be expressed in terms of solving simultaneous equations. However, the fact that the lines might fail to cross at a single point creates serious difficulties for this approach. When more than two calibration signals are present, the problem is over-determined and the system of equations becomes inconsistent if the lines enclose an area rather than intersecting at a point.

As noted in the previous section, the lines would normally be expected to enclose at least a small area. Although a stable solution might be possible using pseudo-inverse techniques [85], the following iterative scheme is preferred.

The technique to be described is based on a gradient search algorithm [102]. Extending the notation used in Chapter 3, $\hat{\sim}_{n}$ is considered to be an estimate of the true sensor position ${\underset{\sim}{n}}_{n}$. The value of ${\underset{\sim}{n}}_{n}$ at the $i$ th step in the iteration is denoted by $\hat{\sim}_{n}(i)$ and the initial value $\hat{u}_{n}(0)$ is set to the intended position of sensor $n$. 
The algorithm adjusts the estimated sensor positions $\hat{u}_{n}$ in an attempt to make them converge to the unknown true positions ${ }_{\sim} n$. Figure 4.4 shows the true and estimated positions of sensor $n$ at steps $i$ and $i+1$ in the iteration. The aim is to update the estimate so that $\hat{u}_{n}(i+1)$ is closer to ${\underset{\sim}{n}}_{n}$ than $\hat{u}_{n}(i)$ was. It follows that if a circle is drawn with $\hat{u}_{n}(i)$ on its circumference and its centre at ${\underset{\sim}{n}}_{n}$ then $\hat{\sim}_{n}(i+1)$ should iie inside the circle as illustrated.

The optimal direction in which to move the estimate is given by the vector difference ${\underset{\sim}{n}}_{n}-\hat{\sim}_{n}(i)$, which is the error between the true value and the estimate, but this vector is unavailable since $u_{n}$ is unknown. However, the components $\epsilon_{\mathrm{nm}}$ of $\mathrm{u}_{\mathrm{n}}$ along the axes of transmission are available and these can be used to guide the estimate towards $u_{n}$ in a non-optimal manner.

The component of $\hat{u}_{n}(i)$ in the direction of $z_{m}$ is $\hat{\epsilon}_{n m}(i)$. Recalling the definition of $\Delta \epsilon_{\mathrm{nm}}$ given in Chapter 3 , the component of the error ${\underset{\sim}{n}}_{n}(i)-u_{n}$ is $\Delta \epsilon_{n m}(i)-\Delta \epsilon_{1 m}(i)$ as shown in the diagram. If the algorithm manages to reduce $\Delta \epsilon_{n m}(i)-\Delta \epsilon_{1 m}(i)$ to zero for all of the $M$ signals simultaneously then $\hat{u}_{n}(i)$ must have become equal to $u_{n}$. More precisely, $\hat{u}_{n}(i)$ will have become equal to the estimate of $u_{n}$ implied by the assumed signal bearings $\mathbf{z}_{m}$ and the measured distances $\epsilon_{\mathrm{nm}}$. 
Y (wavelengths)

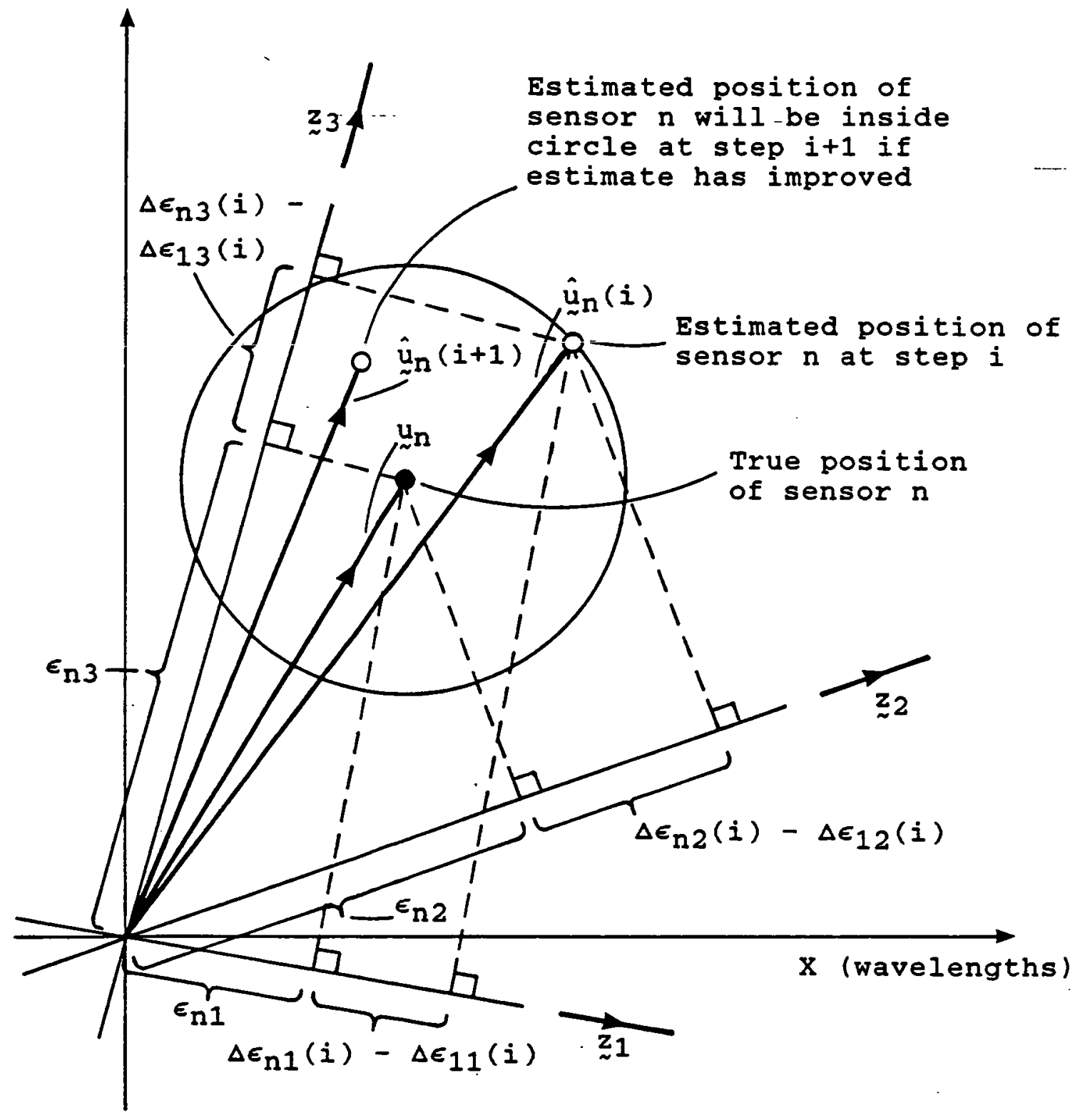

Figure 4.4 Distances for calibration algorithm 
At each step, the algorithm updates the estimated position $\hat{u}_{n}(i)$ of each sensor by adding or subtracting a small amount of each vector $\underset{\sim}{z}$.

$$
\hat{\sim}_{n}(i+1)=\hat{\sim}_{n}(i)-\mu \sum_{m=1}^{M} n_{n m}(i) z_{m}
$$

$\mu$ is a small constant which determines the step size in the iteration. The weight $n_{n m}(i)$ depends on the error $\Delta \epsilon_{\mathrm{nm}}(i)-\Delta \epsilon_{1 \mathrm{~m}}(i)$ in the estimated position of sensor $\mathrm{n}$ along the axis of transmission of signal $m$. If the error is zero then the weight is zero as well. Otherwise, the weight is a small number whose sign is the same as that of the error. For example, if the error in $\hat{u}_{n}(i)$ in the direction of $\mathbf{z}_{\mathrm{m}}$ was positive, $\eta_{n m}(i)$ would also be positive, causing a small amount of $\mathbf{z}_{\mathrm{m}}$ to be subtracted from $\hat{u}_{n}(i)$ in an attempt to reduce the error.

For non-zero errors, the precise magnitude of $n_{n m}(i)$ is related to $\Delta \epsilon_{\mathrm{nm}}(i)-\Delta \epsilon_{1 \mathrm{~m}}(i)$ in some non-linear way through the gradient of the cost function, which is introduced in the next section. However, an understanding of the sign of $n_{\mathrm{nm}}(i)$ is sufficient for the present discussion. 
Although the subtraction of $\mu n_{n m}(i) z_{m}$ from $\hat{\sim}_{n}(i)$ is performed specifically to reduce the error along the axis of signal $\mathrm{m}$, it also changes the error in every other direction except for the one perpendicular to $z_{m}$. In the straightforward case where two signals are being received from perpendicular directions, it is clear that the iteration can minimize the errors along the two axes independently. However, in the general case where the directions are not perpendicular, all $M$ of the $\mu n_{n m}(i) z_{m}$ terms subtracted from $\hat{u}_{n}(i)$ affect all $M$ of the errors $\Delta \epsilon_{\mathrm{nm}}(i+1)-\Delta \epsilon_{1 \mathrm{~m}}(i+1)$ at the next step in the iteration. The aim of the following analysis is to demonstrate that the algorithm converges despite this interaction.

Figure 4.5 shows an enlarged view of the true position of sensor $n$ and the estimated positions at steps $i$ and $i+1$ of the iteration. The large circle, shown only partly in the diagram, is the one discussed earlier. If it can be shown that $\hat{u}_{n}(i+1)$ is consistently placed inside this circle then the estimate must improve at each step.

The argument is as follows. Although $\mathbf{z}_{m}$ can point in any direction, the vector $\left\langle\Delta \epsilon_{\mathrm{nm}}(\mathbf{i})-\Delta \epsilon_{1 \mathrm{~m}}(\mathbf{i})\right\rangle \mathbf{z}_{\mathrm{m}}$ is limited to a range of bearings covering $\pi$ radians. The reason for this is that if $z_{\sim} m$ was replaced with $-z_{\sim} m$, the sign of $\Delta \epsilon_{\mathrm{nm}}(\mathrm{i})-\Delta \epsilon_{1 \mathrm{~m}}(\mathrm{i})$ would change and $\left(\Delta \epsilon_{\mathrm{nm}}(\mathrm{i})-\Delta \epsilon_{1 \mathrm{~m}}(\mathrm{i})\right) \mathbf{z}_{\mathrm{m}}$ 


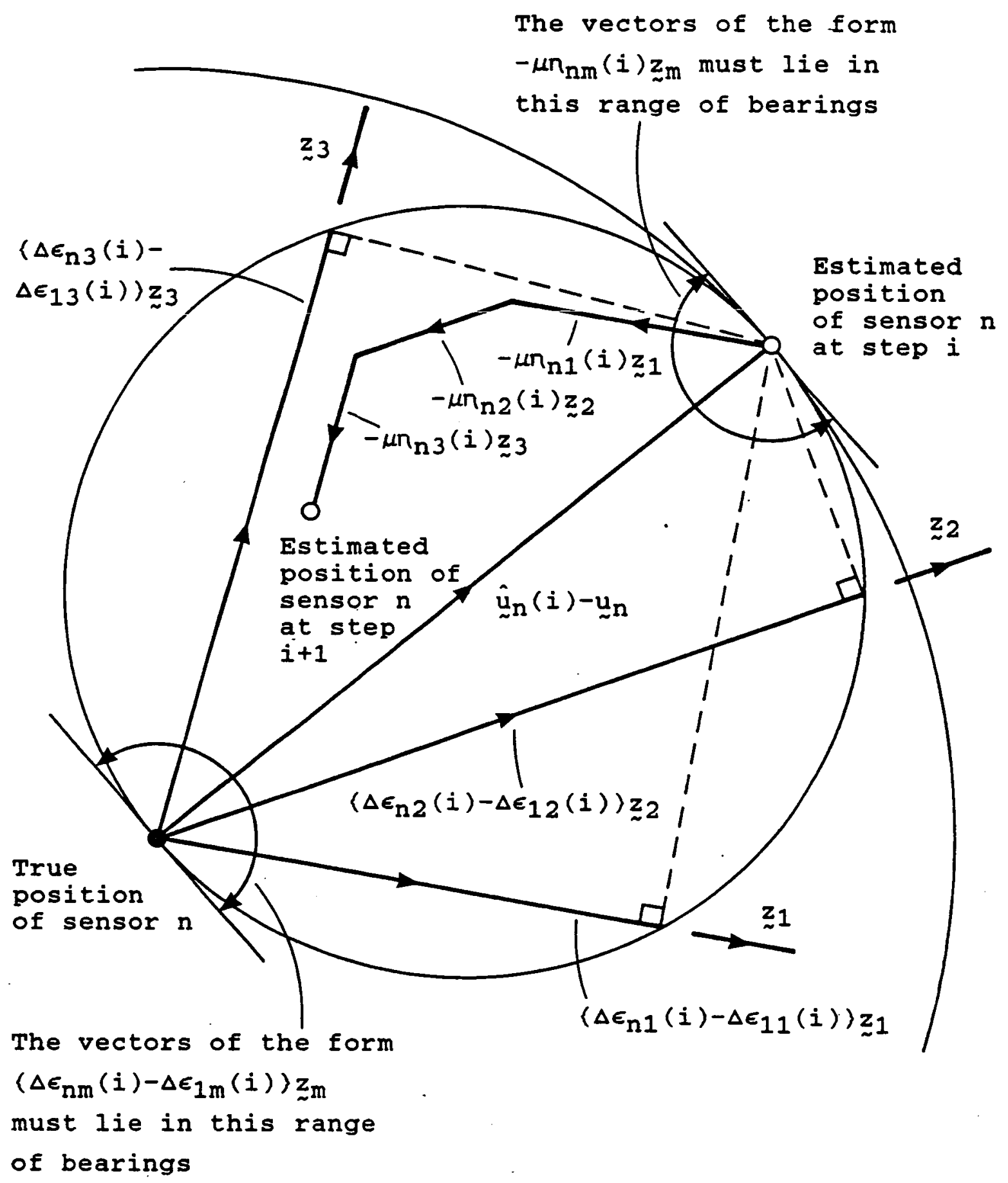

Figure 4.5 Graphical construction for convergence analysis 
would be unaltered.

If the vectors $\left(\Delta \epsilon_{\mathrm{nm}}(i)-\Delta \epsilon_{1 \mathrm{~m}}(i)\right) \boldsymbol{z}_{\mathrm{m}}$ are drawn starting at the true position of sensor $n$, they form chords of a circle as illustrated. The limits of the range of bearings which the vectors can cover are obtained from the tangent to the circle at the true sensor position. At both ends of the range, ${\underset{\sim}{m}}_{m}$ lies along the tangent, causing $\Delta \epsilon_{\mathrm{nm}}(i)-\Delta \epsilon_{1 \mathrm{~m}}(i)$, and hence $n_{\mathrm{nm}}(i)$, to vanish to zero.

When $\Delta \epsilon_{\mathrm{nm}}(i)-\Delta \epsilon_{1 \mathrm{~m}}(i)$ is not equal to zero, the vector $-\mu n_{n m}(i) z_{m}$ always points in the opposite direction to $\left(\Delta \epsilon_{\mathrm{nm}}(i)-\Delta \epsilon_{1 \mathrm{~m}}(i)\right) z_{\mathrm{m}}$ and it follows that the direction of $-\mu n_{n m}(i) z_{m}$ always lies in the range of bearings from which $\left(\Delta \epsilon_{\mathrm{nm}}(\mathrm{i})-\Delta \epsilon_{1 \mathrm{~m}}(\mathrm{i})\right) \mathbf{z}_{\mathrm{m}}$ is excluded.

From (4.1), the vector $\hat{u}_{n}(i+1)-\hat{u}_{n}(i)$ giving the displacement from $\hat{\sim}_{n}(i)$ to $\hat{\sim}_{n}(i+1)$ is the resultant formed by summing $-\mu n_{n m}(i) z_{m}$ over the $M$ signals as illustrated in Figure 4.6(a). Although the precise direction of the resultant depends on the magnitudes of the vectors in the summation, the direction always lies within limits determined only by the set of vectors $\boldsymbol{z}_{m}$ and the signs of the weights $n_{n m}(i)$. In Figure $4.6(b)$, the vectors $-\mu n_{n m}(i) z_{m}$ have been re-drawn so that they all start at the same point. These vectors define a range of bearings, which is from $-\mu n_{n 1}(i) z_{1}$ to $-\mu n_{n 3}(i) z_{3}$ in the 
(a)

Estimated position

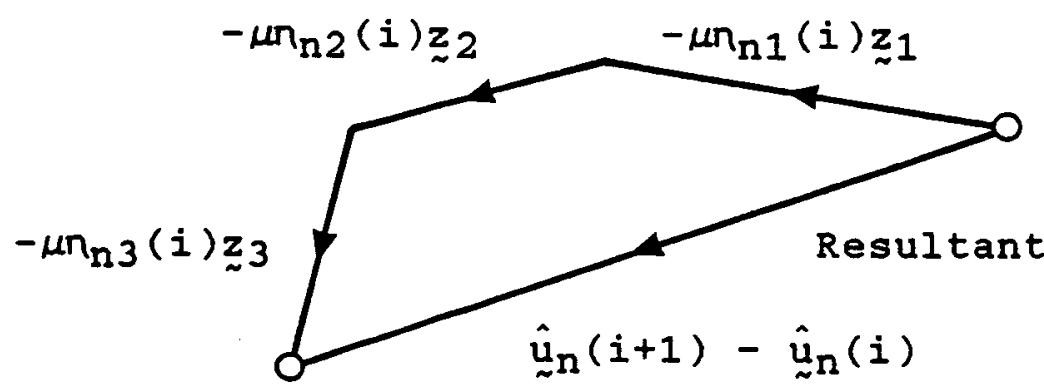
of sensor $n$ at step $i$

Estimated position of sensor $n$ at step $i+1$

(b)

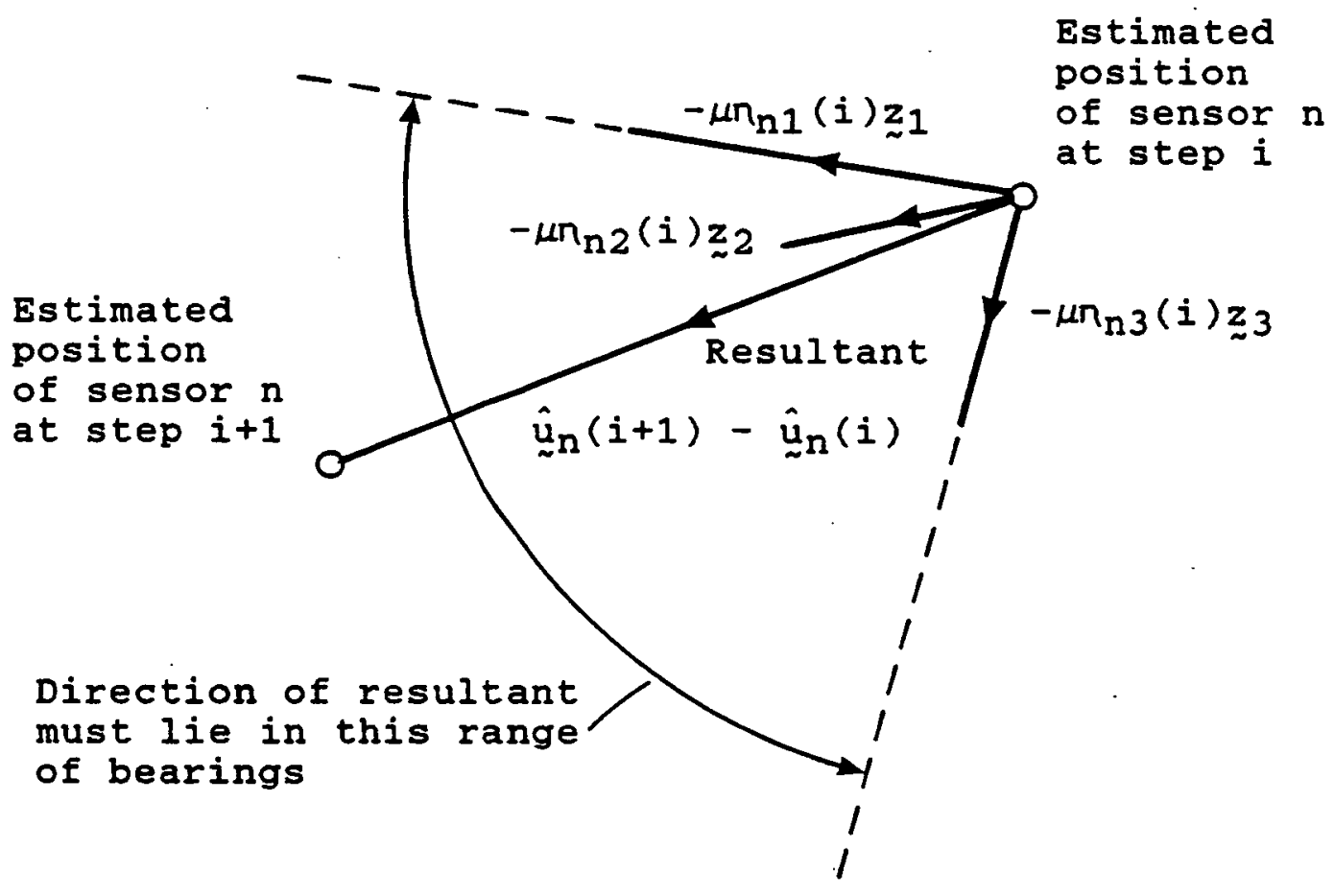

Pigure 4.6 Limits on bearing of resultant 
example, and the direction of the resultant formed by summing the vectors must lie within this range.

Returning to Figure 4.5 , for $\hat{u}_{n}(i+1)$ to lie outside the large circle, there would have to be at least one vector $-\mu n_{n m}(i) z_{m}$ pointing out of the circle. However, this could only happen if $z_{m}$ was a tangent (to both circles), in which case $\Gamma_{n m}(i)$ would be zero, thus excluding $z_{m}$ from the summation in (4.1).

It follows that the resultant can never lie along the tangent to the large circle at the estimated sensor position and must therefore point into the circle. This guarantees that the estimated sensor position moves towards the true one at each step in the iteration.

In this discussion, it has been assumed that the length of the resultant is small compared with the radius of the large circle. Otherwise, the estimated sensor position could overshoot the true one and land outside the circle as shown in Figure 4.7. This problem is most likely to occur close to convergence when the radius, which is the remaining error in the estimate, becomes small.

It follows that the calibration algorithm, in common with many other iterative processes, is sensitive to the choice of the step size $\mu$. A smaller step size causes slower 


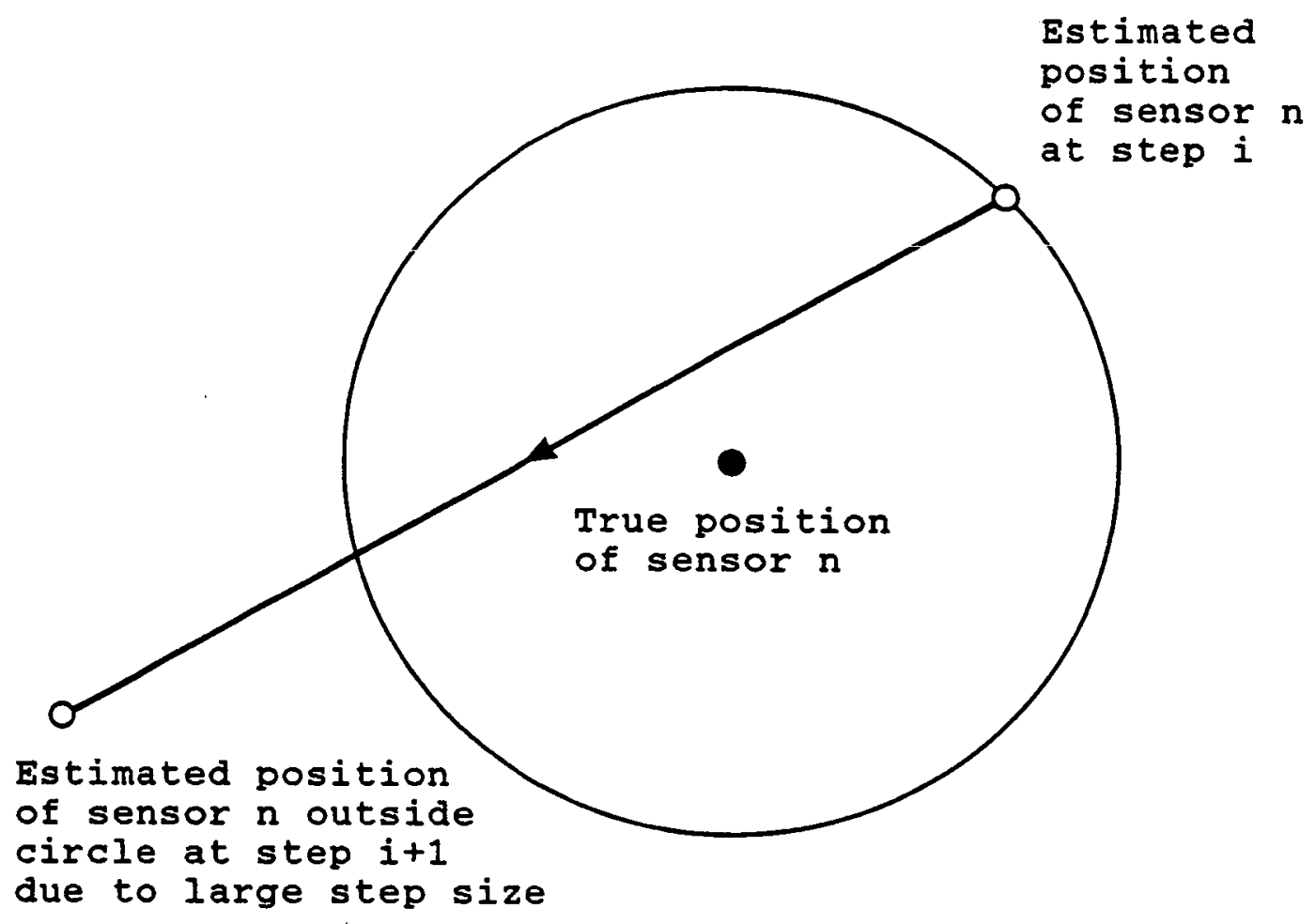

Figure 4.7 Failure due to large step size 
convergence but may reduce the error remaining between the true and estimated sensor positions after calibration.

\subsection{The Array Calibration Algorithm}

This section shows how the signal subspace concepts developed in Chapter 2 can be used to produce the coefficients $n_{n m}(i)$ required by the calibration algorithm. The approach described here produces a calibration algorithm that is closely related to the MUSIC technique for bearing estimation.

While the calibration signals are present, snapshots are obtained from the array and the covariance matrix $\underset{\sim}{\mathbb{R}}$ is calculated in the usual manner. The eigenvalues and eigenvectors of $\underset{\sim}{R}$ are calculated and the eigenvectors corresponding to the $M$ largest eigenvalues form the columns of the matrix $V_{W}$ as before.

The $X$ and $Y$ components of $\hat{u}_{n}(i)$ and $z_{m}$ are denoted by $X\left(\hat{\sim}_{\mathfrak{n}}(i)\right), Y\left(\hat{\sim}_{\mathfrak{n}}(i)\right), X\left({\underset{\sim}{m}}_{m}\right)$, and $Y\left(z_{m}\right)$. For each of the $M$ calibration signals, $\hat{\mathfrak{q}}_{m}(i)$ is the signal vector calculated from the bearing of the signal and the estimated sensor positions $\hat{u}_{n}(i)$. Since the sensor positioning errors are assumed to be small, $\hat{q}_{m}(i)$ is very close to the (unknown) 
signal vector $q_{m}$ which would be obtained using the true sensor positions.

At each step in the iteration, the calibration algorithm must assess the current estimated sensor positions $\hat{\sim}_{n}(i)$ and adjust them so that they improve at the next step. The key is to use the signal subspace approach adopted in MUSIC. The length of the projection of $\hat{q}_{m}(i)$ on to the noise subspace is denoted by $\left\|p_{m}(i)\right\|^{2}$. From Chapter 2, $\left\|p_{m}(i)\right\|^{2}$ is calculated as follows.

$$
\left\|p_{m}(i)\right\|^{2}=\hat{q}_{m}(i) V_{W} V_{\sim}^{H} \hat{q}_{m}(i)
$$

Since $q_{m}$ is a true signal vector, it must lie entirely in the signal subspace. Thus $q_{m}$ has no component in the noise subspace. If there were no sensor positioning errors along the axis of transmission of signal $m, \hat{q}_{m}(i)$ would be equal to $q_{m}$ and would therefore lie entirely in the signal subspace. In this case, $\hat{q}_{m}(i)$ would have no component in the noise subspace and $\left\|p_{m}(i)\right\|^{2}$ would be equal to zero.

$$
\left\|p_{m}(i)\right\|^{2}=0, \quad \hat{q}_{m}(i)=q_{m}
$$

If, however, there are errors along the axis of signal $\mathrm{m}$, $\hat{q}_{\mathbb{M}}(i)$ generally fails to lie completely in the signal subspace and must therefore have some component in the 
noise subspace. It follows that $\left\|p_{m}(i)\right\|^{2}$ is greater than zero.

$$
\left\|p_{m}(i)\right\|^{2}>0, \quad \hat{q}_{m}(i) \neq q_{m}
$$

Thus $\left\|p_{m}(i)\right\|^{2}$ is a measure of the sensor positioning errors along the axis of transmission of signal $m$, being (theoretically) equal to zero when there are no errors in that direction. The cost function $J(i)$ is defined to be the sum of $\left\|p_{m}(i)\right\|^{2}$ over all M signals.

$$
J(i)=\sum_{m=1}^{M}\left\|p_{m}(i)\right\|^{2}
$$

The algorithm manipulates the estimated sensor positions $\hat{u}_{n}(i)$ in way which minimizes $J(i)$, thus reducing the $\left\|p_{m}(i)\right\|^{2}$ terms together. In terms of the graphical constructions used earlier, varying $\hat{u}_{n}(i)$ so as to minimize $\left\|\operatorname{\sim p}_{m}(i)\right\|^{2}$ tends to steer the sensor on to the dashed line perpendicular to $\mathbf{z}_{\mathrm{m}}$ passing through the point $\epsilon_{n m} z_{m} \cdot \quad$ Similarly, minimizing $J(i)$ guides the sensor towards the point of intersection of the M dashed lines, thus achieving the triangulation.

If the lines enclose an area rather than crossing at a point then the sensor cannot lie on all of the dashed 
lines at the same time and the $\left\|p_{m}(i)\right\|^{2}$ terms cannot all be zero simultaneously. In this case, it is impossible to reduce $J(i)$ to zero.

The iteration used to minimize $J(i)$ is a gradient search algorithm. The $N$ estimated sensor positions $\hat{\sim}_{n}(i)$ are updated in parallel as follows.

$$
\hat{u}_{n}(i+1)=\hat{u}_{n}(i)-\mu \frac{\partial}{\partial \hat{u}_{n}(i)} J(i)
$$

To implement (4.6), it is necessary to differentiate the cost function $J(i)$ with respect to each of the estimated sensor positions $\hat{u}_{n}(i)$. This differentiation is possible since $J(i)$ is a function of the $\left\|p_{m}(i)\right\|^{2}$ terms, which are themselves related to the positions $\hat{\sim}_{n}(i)$ through the signal vectors $\hat{q}_{m}(i)$.

The first step is to differentiate $\left\|p_{m}(i)\right\|^{2}$ with respect to $X\left(\hat{u}_{n}(i)\right)$ and $Y\left(\hat{u}_{n}(i)\right)$. The matrix multiplications in (4.2) can be expanded as follows.

$$
\left\|p_{m}\right\|^{2}=\sum_{h=1}^{N-M}\left[\left\{\sum_{p=1}^{N} \hat{q}_{p m}^{*} v_{p h}\right\}\left\{\sum_{p=1}^{N} v_{p h}^{*} \hat{q}_{p m}\right\}\right]
$$


In the interests of compactness, the iteration count $i$ has been omitted. $\quad \hat{q}_{p m}$ is the $p^{\text {th }}$ element of the signal vector $\hat{q}_{m}(i)$ and $v_{p h}$ is an element of ${\underset{\sim}{W}}_{W}$. since $\hat{q}_{n m}$ and $\hat{q}_{n m}^{\star}$ are the only terms affected by $X\left(\hat{u}_{n}(i)\right)$ and $Y\left(\hat{u}_{n}(i)\right)$, it is sufficient to consider only the influence of these terms when forming the partial derivatives. By inspection, the coefficients of $\hat{q}_{n m}$ and $\hat{q}_{n m}^{*}$ in $\left\|p_{m}\right\|^{2}$ are as foliows.

$$
\begin{aligned}
& \text { Coefficient of } \hat{q}_{n m}: \sum_{h=1}^{N-M}\left\{v_{n h}^{*} \sum_{p=1}^{N} \hat{q}_{p m}^{*} v_{p h}\right\} \\
& \text { Coefficient of } \hat{q}_{n m}^{*}: \sum_{h=1}^{N-M}\left\{v_{n h} \sum_{p=1}^{N} v_{p h}^{*} \hat{q}_{p m}\right\}
\end{aligned}
$$

Notice that when $p$ is equal to $n$ in $(4.8)$, the coefficient of $\hat{q}_{n m}$ contains a reference to $\hat{q}_{n m}^{*}$. However, multiplying the coefficient by $\hat{q}_{n m}$ produces a constant.

$$
\left(v_{n h}^{*} \hat{q}_{n m}^{*} v_{n h}\right) \hat{q}_{n m}=\left|\hat{q}_{n m}\right|^{2}\left|v_{n h}\right|^{2}=\left|v_{n h}\right|^{2}
$$

The same result is obtained when $p$ is equal to $n$ in (4.9). Since the derivative of a constant is zero, the differentiation can be simplified by excluding the constants altogether. The partial derivative of $\left\|p_{m}(i)\right\|^{2}$ with respect to $x\left(\hat{u}_{n}(i)\right)$ is then as follows. 


$$
\begin{aligned}
\frac{\partial}{\partial X\left(\hat{\sim}_{n}\right)}\left\|\underline{\sim}_{m}\right\|^{2} & =\left[\sum_{h=1}^{N-M}\left\{v_{n h}^{*} \sum_{\substack{p=1 \\
p \neq n}}^{N} \hat{q}_{p m}^{*}{ }^{*} p h\right\}\right] \frac{\partial}{\partial x\left(\hat{u}_{n}\right)} \hat{q}_{n m} \\
& +\left[\sum_{h=1}^{N-M}\left\{v_{n h} \sum_{\substack{p=1 \\
p \neq n}}^{N} v_{p h}^{*} \hat{q}_{p m}\right\}\right] \frac{\partial}{\partial x\left(\hat{u}_{n}\right)} \hat{q}_{n m}^{*}
\end{aligned}
$$

$\hat{q}_{n m}$ is related to $X\left(\hat{u}_{n}\right)$ and $Y\left(\hat{u}_{n}\right)$ as follows.

$$
\begin{aligned}
& \hat{\mathrm{q}}_{\mathrm{nm}}=\exp \left[j \hat{\psi}_{\mathrm{nm}}\right] \\
& =\exp \left[j 2 \pi \hat{\epsilon}_{\mathrm{nm}}\right] \\
& =\exp \left[j 2 \pi \hat{\sim}_{\mathrm{n}} \cdot \boldsymbol{z}_{\mathrm{m}}\right] \\
& =\exp \left[j 2 \pi\left\langle X\left(\hat{\sim}_{n}\right) X\left(\underline{\sim}_{m}\right)+Y\left({\underset{\sim}{\mathrm{u}}}_{n}\right) Y\left({\underset{\sim}{m}}_{m}\right)\right\}\right]
\end{aligned}
$$

Having expressed $\hat{q}_{n m}$ in this form, it is straightforward to find the partial derivatives of $\hat{q}_{n m}$ and $\hat{q}_{n m}^{*}$ with respect to $x\left(\hat{\sim}_{n}\right)$.

$$
\begin{aligned}
& \frac{\partial}{\partial X\left(\hat{\sim}_{n}\right)} \hat{q}_{n m}=j 2 \pi x\left({\underset{\sim}{m}}_{m}\right) \hat{q}_{n m} \\
& \frac{\partial}{\partial X\left(\hat{\sim}_{n}\right)} \hat{q}_{n m}^{*}=-j 2 \pi x\left({\underset{\sim}{m}}_{m}\right) \hat{q}_{n m}^{*}
\end{aligned}
$$

The partial derivative of $\left\|\underline{\sim}_{m}\right\|^{2}$ with respect to $x\left(\hat{\sim}_{n}\right)$ is obtained by substituting (4.13) and (4.14) into (4.11). 


$$
\begin{gathered}
\frac{\partial}{\partial X\left(\hat{u}_{n}\right)}\left\|\sim_{m}\right\|^{2}=j 2 \pi x\left(\underset{\sim}{z_{m}}\right)\left[\hat{q}_{n m} \sum_{h=1}^{N-M}\left\{v_{n h}^{*} \sum_{\substack{p=1 \\
p \neq n}}^{N} \hat{q}_{p m}^{*} v_{p h}\right\}\right. \\
\left.-\hat{q}_{n m}^{*} \sum_{h=1}^{N-M}\left\{v_{n h} \sum_{\substack{p=1 \\
p \neq n}}^{N} v_{p h}^{*} \hat{q}_{p m}\right\}\right]
\end{gathered}
$$

Noting that the two expressions in the square brackets are complex conjugates of each other, (4.15) can be re-written as follows.

$$
\frac{\partial}{\partial X\left(\hat{\sim}_{n}\right)}\left\|p_{m}\right\|^{2}=-4 \pi x\left(z_{m}\right) \operatorname{Im}\left[\hat{q}_{n m} \sum_{h=1}^{N-M}\left\{v_{n h}^{*} \sum_{\substack{p=1 \\ p \neq n}}^{N} \hat{q}_{p m}^{*} v_{p h}\right\}\right]
$$

Im[] denotes the imaginary part (which is itself a real number). The partial derivative with respect to $Y\left(\hat{\sim}_{n}(i)\right)$ is identical to the right hand side of (4.16) except that $\mathrm{X}\left(\mathbf{z}_{\mathrm{m}}\right)$ is replaced with $\mathrm{Y}\left(\mathbf{z}_{\mathrm{m}}\right)$. Re-introducing the iteration count $i, n_{n m}(i)$ is defined as follows.

$$
n_{n m}(i)=-4 \pi I m\left[\hat{q}_{n m}(i) \sum_{h=1}^{N-M}\left\{v_{n h}^{\star} \sum_{\substack{p=1 \\ p \neq n}}^{N} \hat{q}_{p m}^{*}(i) v_{p h}\right\}\right]
$$


The partial derivatives of $\left\|\underline{\sim}_{m}(i)\right\|^{2}$ with respect to $X\left(\hat{u}_{n}(i)\right)$ and $Y\left(\hat{u}_{n}(i)\right)$ can now be expressed in terms of $X\left(z_{m}\right), Y\left(z_{m}\right)$, and $n_{n m}(i)$.

$$
\begin{aligned}
& \frac{\partial}{\partial X\left(\underline{\sim}_{n}(i)\right)} \cdot\left\|\underline{\sim}_{m}(i)\right\|^{2}=n_{n m}(i) X\left(\sim_{\sim} m\right) \\
& \frac{a}{\partial Y\left(\hat{u}_{n}(i)\right)}\left\|\underline{\sim}_{m}(i)\right\|^{2}=n_{n m}(i) Y\left({\underset{\sim}{m}}_{m}\right)
\end{aligned}
$$

Combining (4.18) and (4.19), the derivative with respect to the vector $\hat{u}_{n}(i)$ is as follows.

$$
\frac{\partial}{\partial \hat{u}_{n}(i)}\left\|\underline{\sim}_{m}(i)\right\|^{2}=n_{n m}(i) z_{\sim}
$$

Recalling the definition of the cost function $J(i)$ given in (4.5), the derivative of $J(i)$ is the sum of the derivatives of the individual $\left\|p_{m}(i)\right\|^{2}$ terms.

$$
\frac{\partial}{\partial \hat{\underline{u}}_{n}(i)} J(i)=\sum_{m=1}^{M} \frac{\partial}{\partial \hat{u}_{n}(i)}\left\|\underline{\sim}_{m}(i)\right\|^{2}=\sum_{m=1}^{M} n_{n m}(i) z_{\sim}
$$

The final step in the derivation is to substitute (4.21) into the gradient search algorithm defined in (4.6). 


$$
\hat{\sim}_{n}(i+1)=\hat{u}_{n}(i)-\mu \sum_{m=1}^{M} n_{n m}(i) \underset{\sim m}{z}
$$

When combined with the definition of $\pi_{n m}$ (i) given in (4.17), equation (4.22) specifies how the estimated sensor positions should be updated during the array calibration procedure. Notice that (4.22) is identical to the scheme originally proposed in (4.1), although the form of the weights $n_{n m}(i)$ was unknown at that point. Figure 4.8 provides a summary of the algorithm.

The remainder of this chapter demonstrates the performance of the array calibration algorithm using computer simulations.

\subsection{Computer Simulation of the Array Calibration Algorithm}

The simulation system includes a program which models the calibration algorithm described in this chapter. The main inputs to the program are the intended sensor positions $\hat{u}_{n}$, the vectors $z_{m}$ describing the bearings of the calibration signals, and the eigenvectors $\mathbb{\sim}_{n}$ of a covariance matrix formed while the calibration signals are present. The main output from the program is a set of 


\section{Initialize}

$$
\begin{aligned}
& \text { for }{\underset{\sim}{\hat{u}_{n}}(0)}_{n}\left(0,=\dot{\hat{\dot{u}}}_{n}, N\right. \\
& i=0
\end{aligned}
$$

\section{Update sensor positions}

for $n=1, \ldots, N$

$$
\begin{aligned}
& \text { for } m_{\hat{q}_{n m}(i)}=1, \cdots, M \\
& n_{n m}(i)=-4 \pi I m\left[\hat{q}_{n m}(i) \sum_{h=1}^{N-M}\left\{v_{n h}^{*} \sum_{\substack{p=1 \\
p \neq n}}^{N} \hat{q}_{p m}^{*}(i) v_{p h}\right\}\right] \\
& \hat{u}_{n}(i+1)=\hat{u}_{n}(i)-\mu \sum_{m=1}^{M} n_{n m}(i) z_{\sim}
\end{aligned}
$$$$
\mathbf{p} \neq \mathbf{n}
$$

\section{Evaluate cost function}

for $m=1, \ldots, M$

$\left\|p_{m}(i)\right\|^{2}=\hat{q}_{m}(i) V_{W} \underset{\sim}{V} \hat{q}_{m}(i)$

$J(i)=\sum_{m=1}^{M}\left\|p_{m}(i)\right\|^{2}$

$i=i+1$

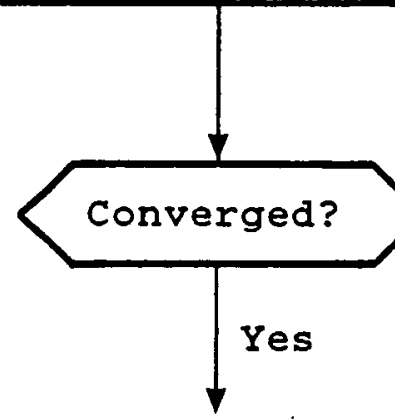

Figure 4.8 The array calibration algorithm 
estimated sensor positions $\hat{u}_{n}(i)$, which are approximations of the unknown true sensor positions ${\underset{\sim}{n}}_{n}$.

Although the true sensor positions would be unknown in a real application, they are available in the simulation system. The calibration program takes the true positions ${\underset{\sim}{n}}_{\mathrm{n}}$ as additional inputs and measures the errors between these positions and the estimates $\dot{u}_{n}(i)$. The overall error between the two sets of positions after the $i$ th step in the iteration is denoted by $D(i)$, which is defined to be the magnitude $\left\|u_{n}-\hat{u}_{n}(i)\right\|$ summed over the $N$ sensors.

$$
D(i)=\sum_{n=1}^{N}\left\|u_{n}-\hat{u}_{n}(i)\right\|
$$

The program outputs the error $D(i)$ and the cost function $J(i)$ after each step, thus allowing $D(i)$ and $J(i)$ to be plotted against the step number, $i$. This provides a means of investigating the convergence properties of the calibration algorithm.

The covergence test shown in Figure 4.8 is implemented by stopping the iteration whenever the counter $i$ reaches a specified limit, the cost function $J(i)$ falls below a given value, or the improvement $|J(i-1)-J(i)|$ in the cost function falls below a certain threshold. 
All of the simulation results presented in this chapter are based on the circular array used in Chapters 2 and 3. This array defines the intended sensor positions $\hat{u}_{n}$. A set of true sensor positions ${\underset{\sim}{n}}_{n}$ was formed by modelling the process of setting up the array with the variance of the sensor positions $\sigma_{\mathrm{p}}^{2}$ set to 0.001 . This was performed using the program described in chapter 3 , resulting in an initial overall error of $\bar{D}(0)=0 . \overline{2} 1 \dot{8}$ wavelengths.

The first set of results consists of a series of bearing spectra which demonstrate the improvements obtained using the calibration algorithm. The array was calibrated using three signals with bearings of $-108,18$, and 90 degrees and relative frequencies of $0.999,1.0$, and 1.001 respectively. All three signals had a power of $20 \mathrm{~dB}$ and an initial phase angle of zero degrees. The noise power. $\sigma_{\mathrm{W}}^{2}$ was set to $10 \mathrm{~dB}$.

Figures 4.9 and 4.10 show MUSIC bearing spectra for the calibration signals themselves. The spectra were generated using the same covariance matrix but different sets of sensor positions. The covariance matrix was formed using 1600 snapshots obtained at a sampling rate of 3.4567 samples per cycle. In the bearing spectrum obtained from the true sensor positions, which is shown in Figure $4.9(a)$, there are no errors in the bearings of the 
(a) $\log _{10} P_{\mathrm{mu}}(\theta)$

50

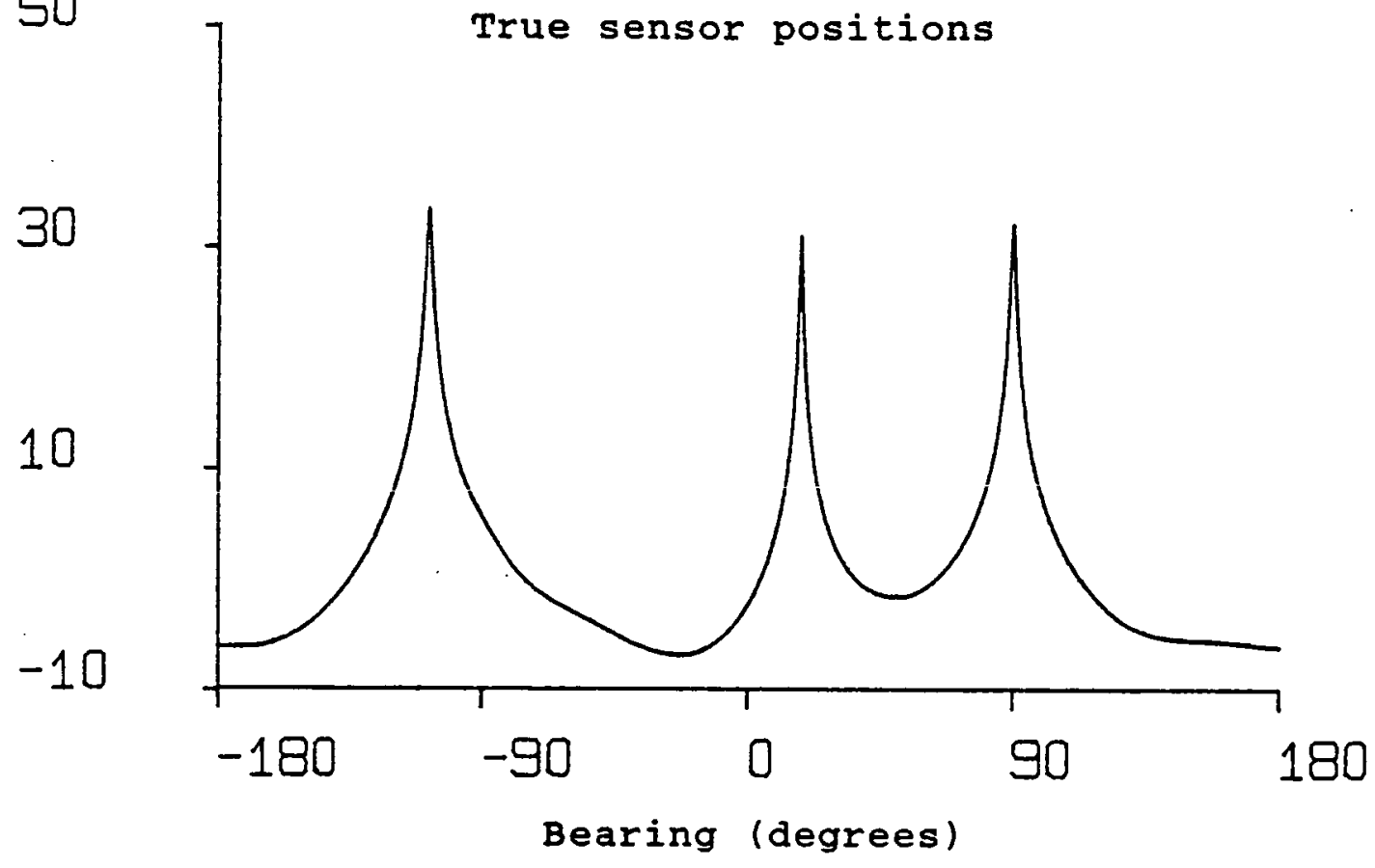

(b) ${ }^{0} 0 \log _{10} P_{\mathrm{mu}}(\theta)$

50

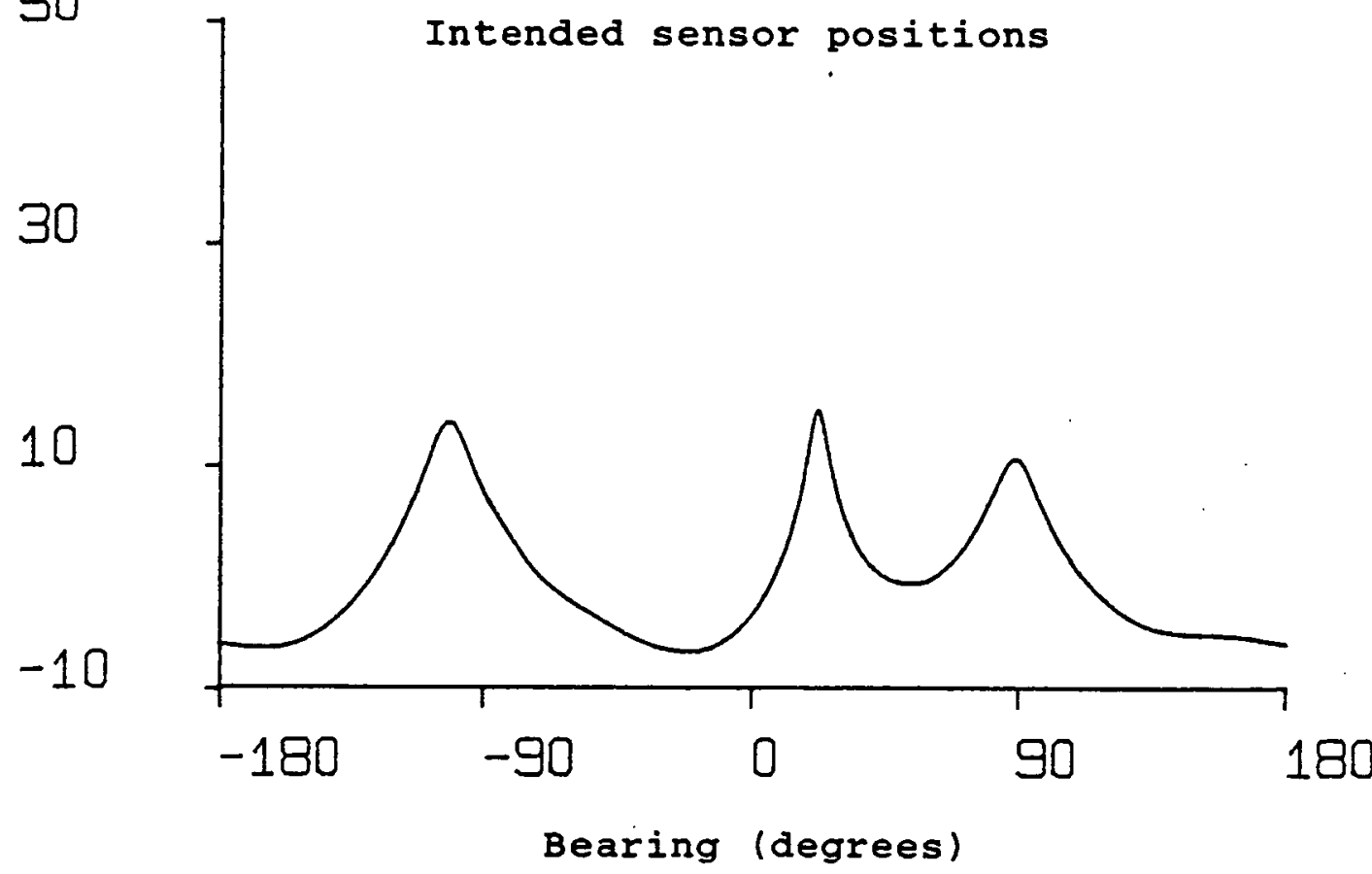

Figure 4.9 MUSIC bearing spectra for calibration signals 
(a) $10 \log _{10} P_{\mathrm{mu}}(\theta)$

50

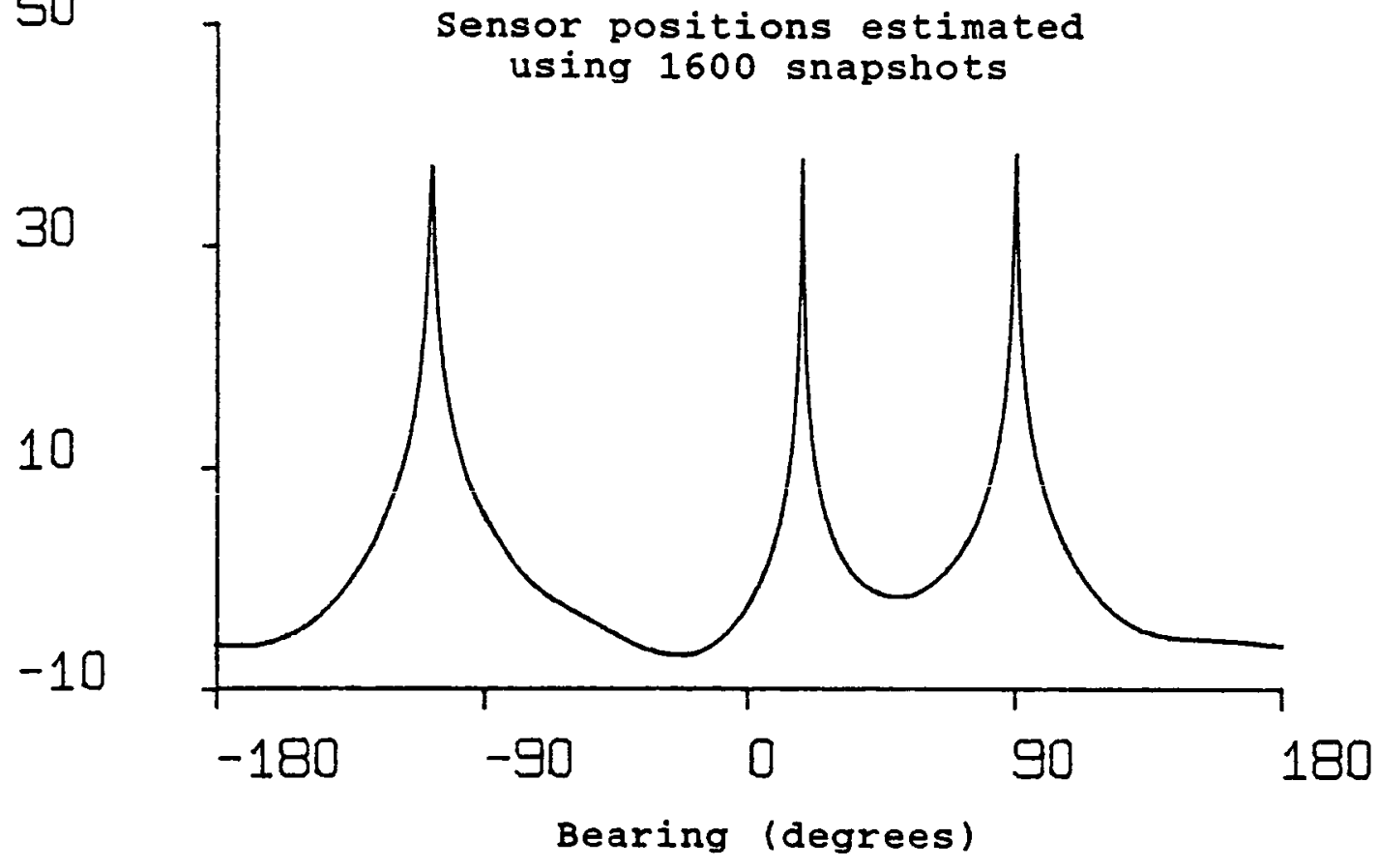

(b) $10 \log _{10} P_{\mathrm{mu}}(\theta)$

50

Sensor positions estimated

using 6400 snapshots

30

10

$-10$

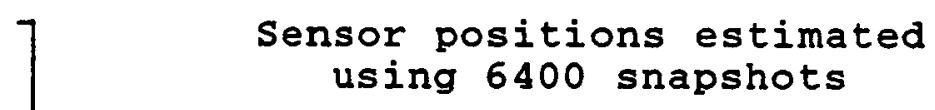

Figure 4.10 MUSIC bearing spectra for calibration signals 
signals. It should be stressed, however, that this spectrum would only be available if the true sensor positions were known.

Figure 4.9 (b) shows the spectrum obtained using the intended sensor positions. The bearings of the peaks are -102, 22.5, and 88.5 degrees, giving an overall bearing error of 12 degrees summed over the three signals. This is the bearing spectrum which would be produced by a conventional (i.e. uncalibrated) bearing estimation system.

The bearing spectrum shown in Figure $4.10(a)$ was generated using a set of estimated sensor positions produced by the calibration algorithm with the step size $\mu$ set to 0.005 . The iteration stopped after 627 steps when $|J(i-1)-J(i)|$ fell below the specified limit of $10^{-12}$. The final value of the cost function $J(i)$ was $4.90 \times 10^{-4}$ and the algorithm reduced the overall error from 0.218 to 0.0299 wavelengths. The bearings of the peaks in the resulting spectrum are all correct (within the 0.5 degree granularity of the plot).

The calibration process was repeated with a covariance matrix formed using 6400 snapshots. All of the other parameters remained unchanged. The algorithm terminated after 455 steps, $|J(i-1)-J(i)|$ having fallen below 10-12. The improved quality of the covariance matrix 
allowed the algorithm to form a better approximation of the true sensor positions. The final value of the cost function $J(i)$ was $7.52 \times 10^{-5}$ and the overall error $D(i)$ was reduced from 0.218 to 0.00896 wavelengths. Figure 4.10(b) shows the bearing spectrum obtained by combining the estimated sensor positions with the original covariance matrix. The bearings of the peaks in the spectrum are ali correct.

As anticipated, the use of the estimated sensor positions in place of the intended ones yielded improved bearing spectra for signals with unknown bearings. In Figures 4.11 and 4.12 , the three calibration signals have been replaced with two new signals with bearings of -45 and -18 degrees and relative frequencies of 1.001 and 1.0 respectively. Both signals had a power of $20 \mathrm{~dB}$ and an initial phase angle of zero degrees. The noise power was $O d B$ and the covariance matrix was formed using 500 snapshots obtained at a sampling rate of 3.4567 samples per cycle. Figure 4.11 (a) shows the bearing spectrum which would be produced if the true sensor positions were available.

The spectrum obtained using the intended sensor positions is shown in Figure $4.11(\mathrm{~b})$. The peaks are at -48 and -13.5 degrees, giving an overall bearing error of 7.5 degrees. Figure 4.12 demonstrates the improvement 
(a) $10 \log _{10} \mathrm{P}_{\mathrm{mu}}(\theta)$

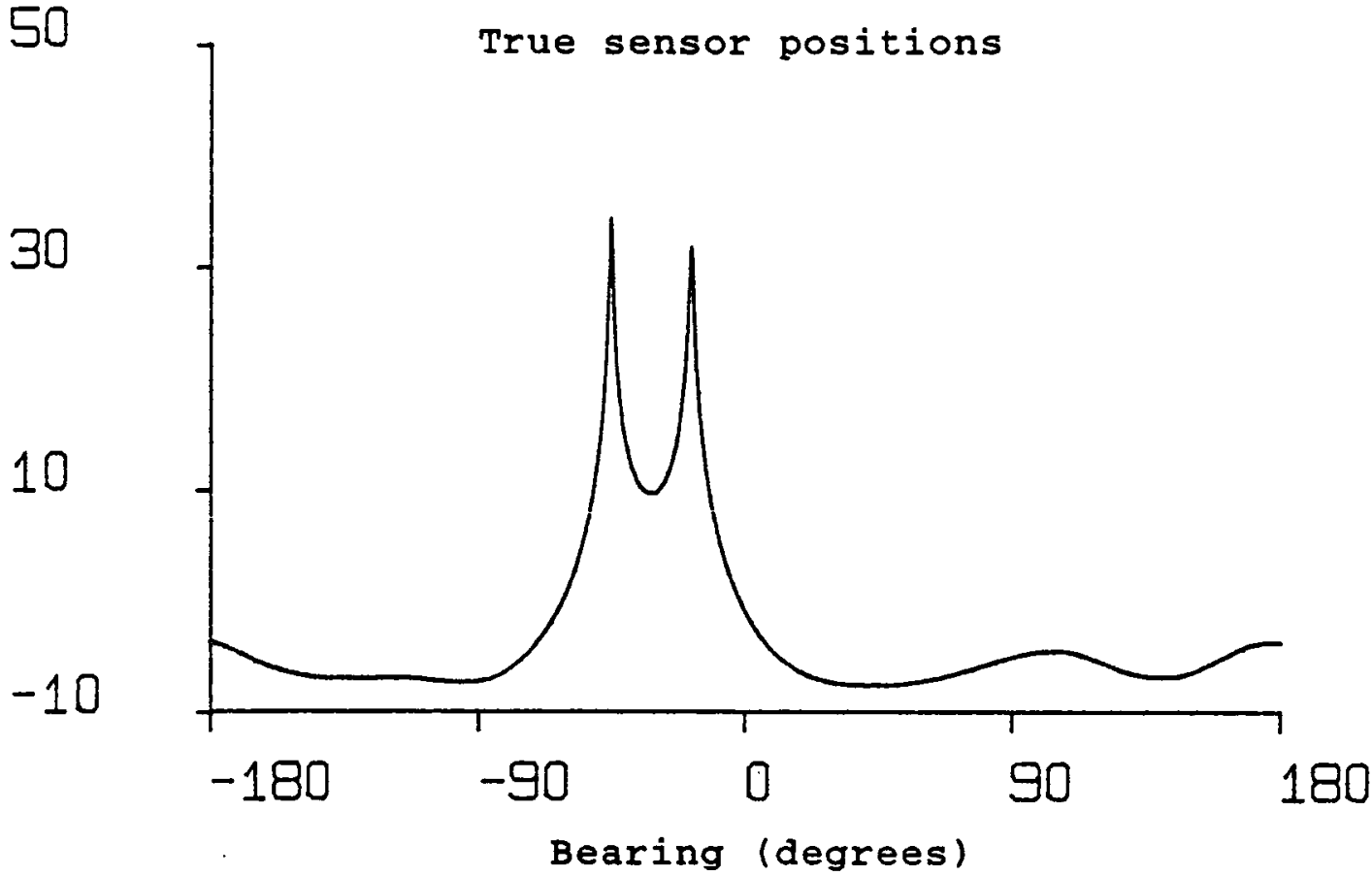

(b) $10 \log _{10} P_{m u}(\theta)$

50

30

10

$-10$

Intended sensor positions

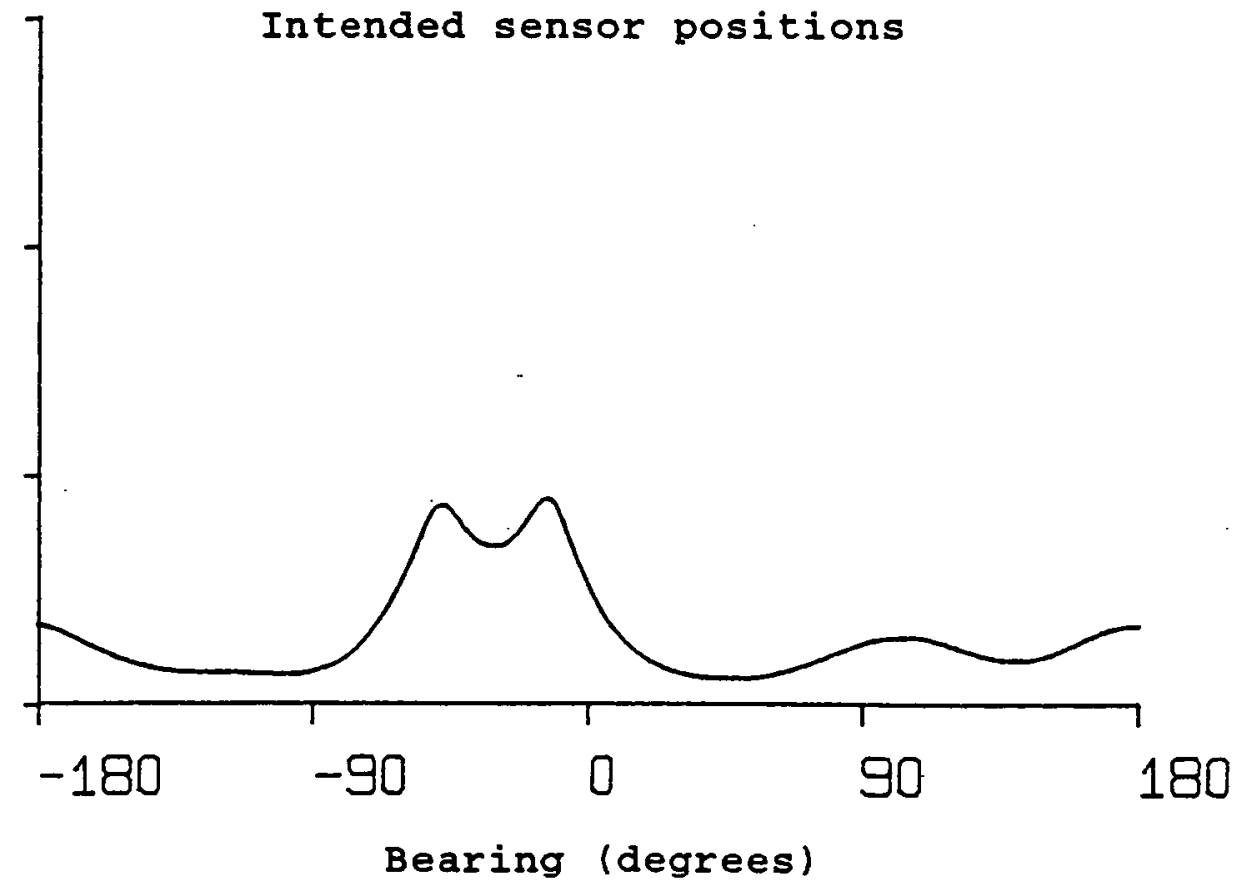

Figure 4.11 MUSIC bearing spectra for signals with unknown bearings 
(a) $\log _{10} \mathrm{P}_{\mathrm{mu}}(\theta)$

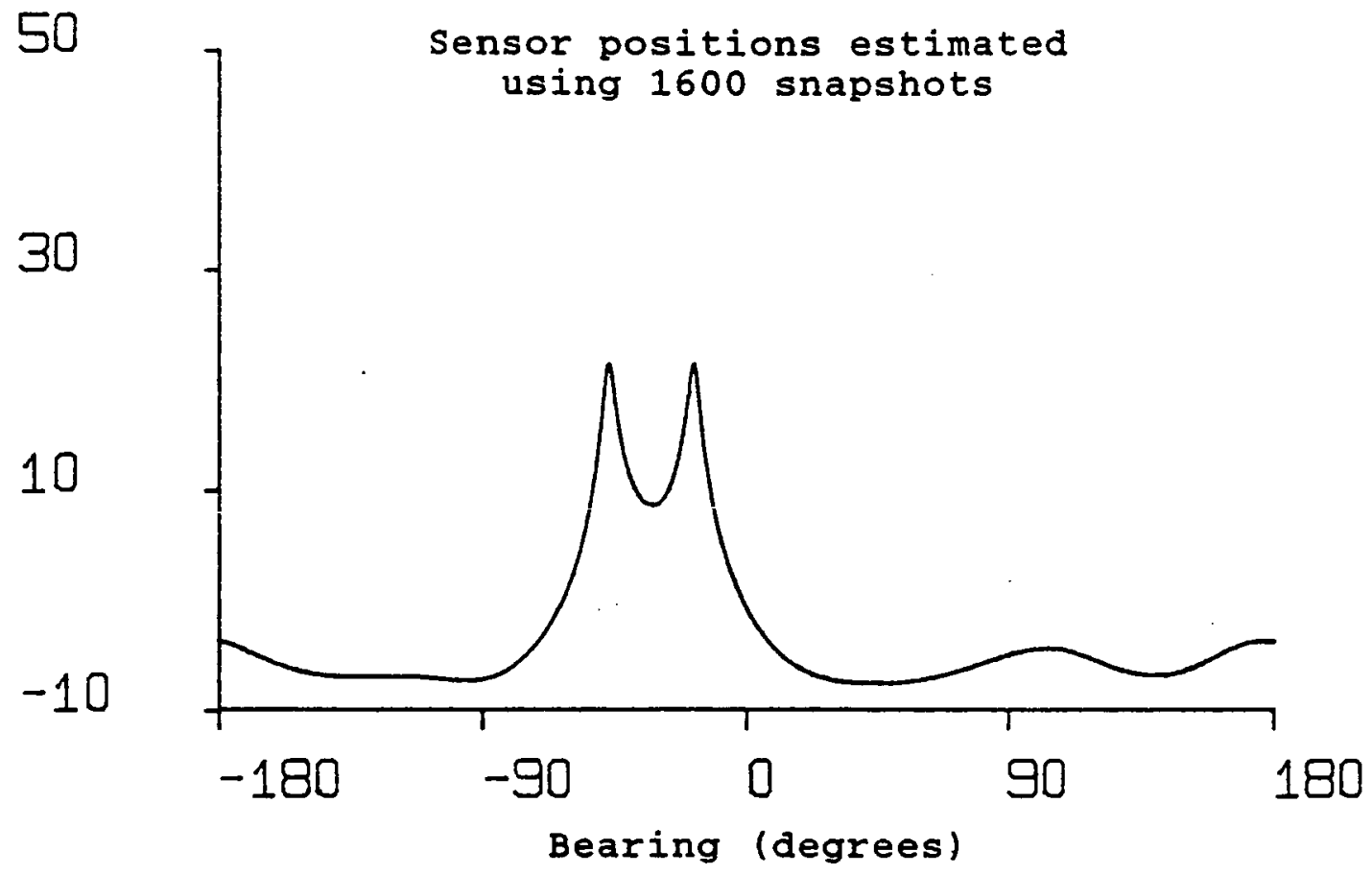

(b) $10 \log _{10} P_{\mathrm{mu}}(\theta)$

50

30
10
-10
Sensor positions estimated using 6400 snapshots

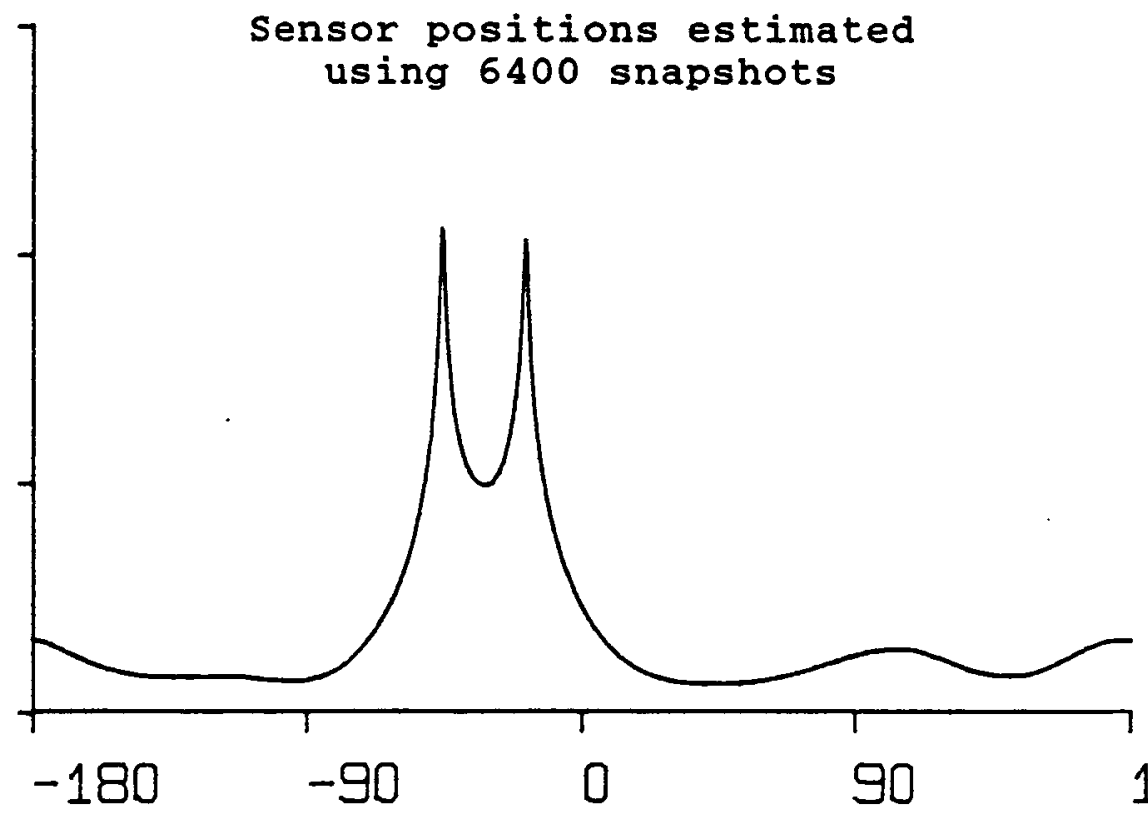

Bearing (degrees)

Figure 4.12 MUSIC bearing spectra for signals with unknown bearings 
achieved by replacing the intended sensor positions with the estimated positions derived from the three calibration signals. Using the estimates obtained from 1600 snapshots, the bearings of the peaks are -46 and -17.5 degrees, meaning that the overall bearing error has been reduced from 7.5 to 1.5 degrees. When the estimated sensor positions based on 6400 snapshots are used, the bearings of the peaks are correct (to within 0.5 degrees).

The next set of results investigates the behaviour of the cost function $J(i)$ and the error $D(i)$ while the iteration is converging. It should be stressed that $D(i)$ would not be available in a real application since the calculation involves the true sensor positions.

As noted at the start of the chapter, the use of three or more calibration signals has the advantage that errors in the bearings of the signals can be detected. If there are only two signals present, such errors cannot be discovered. This effect is demonstrated by the simulation results.

In Figure 4.13, the cost function $J(i)$ and the overall error $D(i)$ are plotted on a logarithmic scale against the step number $i$. The error $D(i)$ is normalized so that the original error $D(O)$ is at zero on the vertical axis. There were two calibration signals with bearings of 18 and 
(a) $\operatorname{lol} \log _{10} \mathrm{~J}(\mathrm{i})$

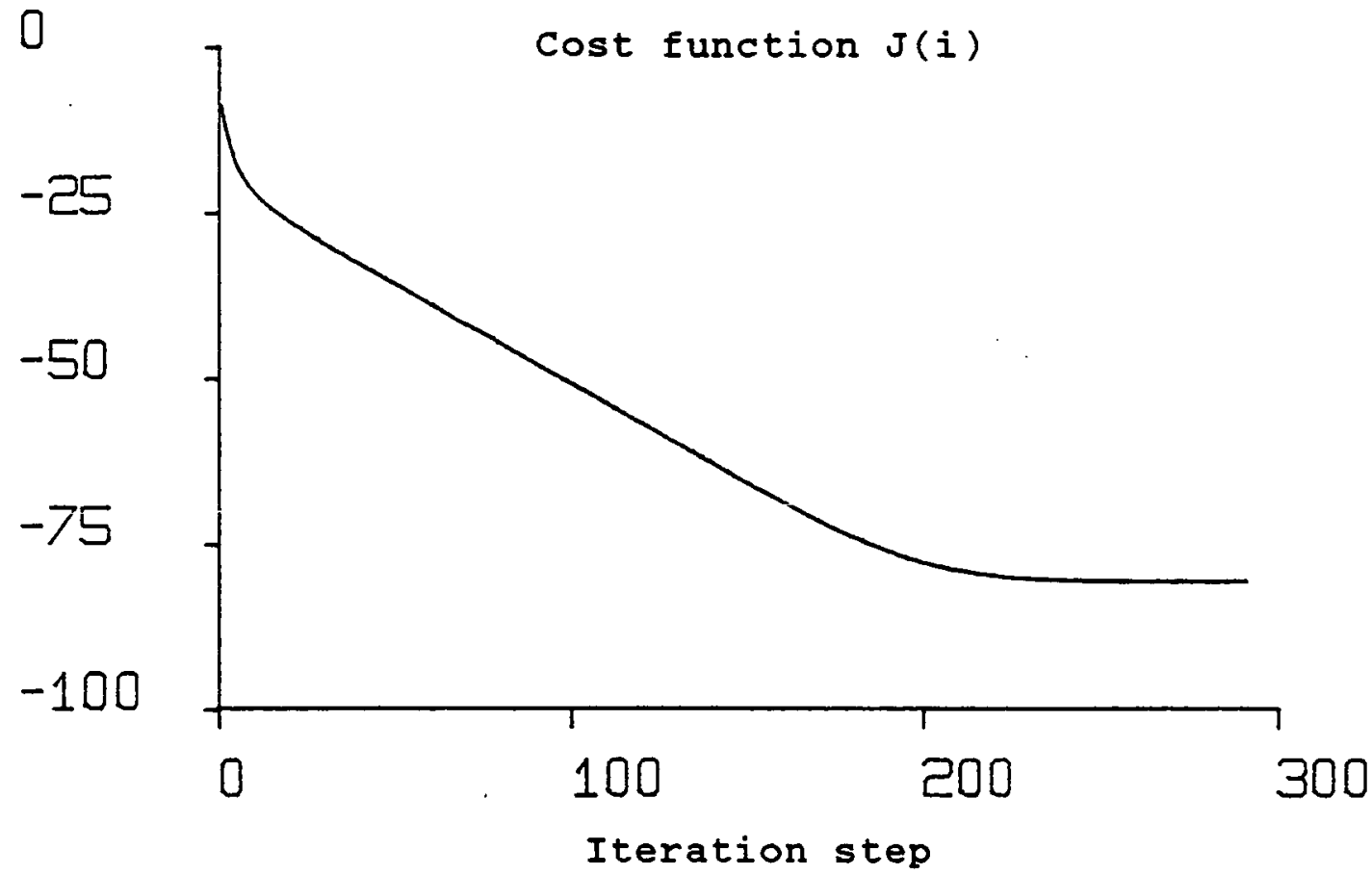

(b) $10 \log _{10} \mathrm{D}(\mathrm{i})$

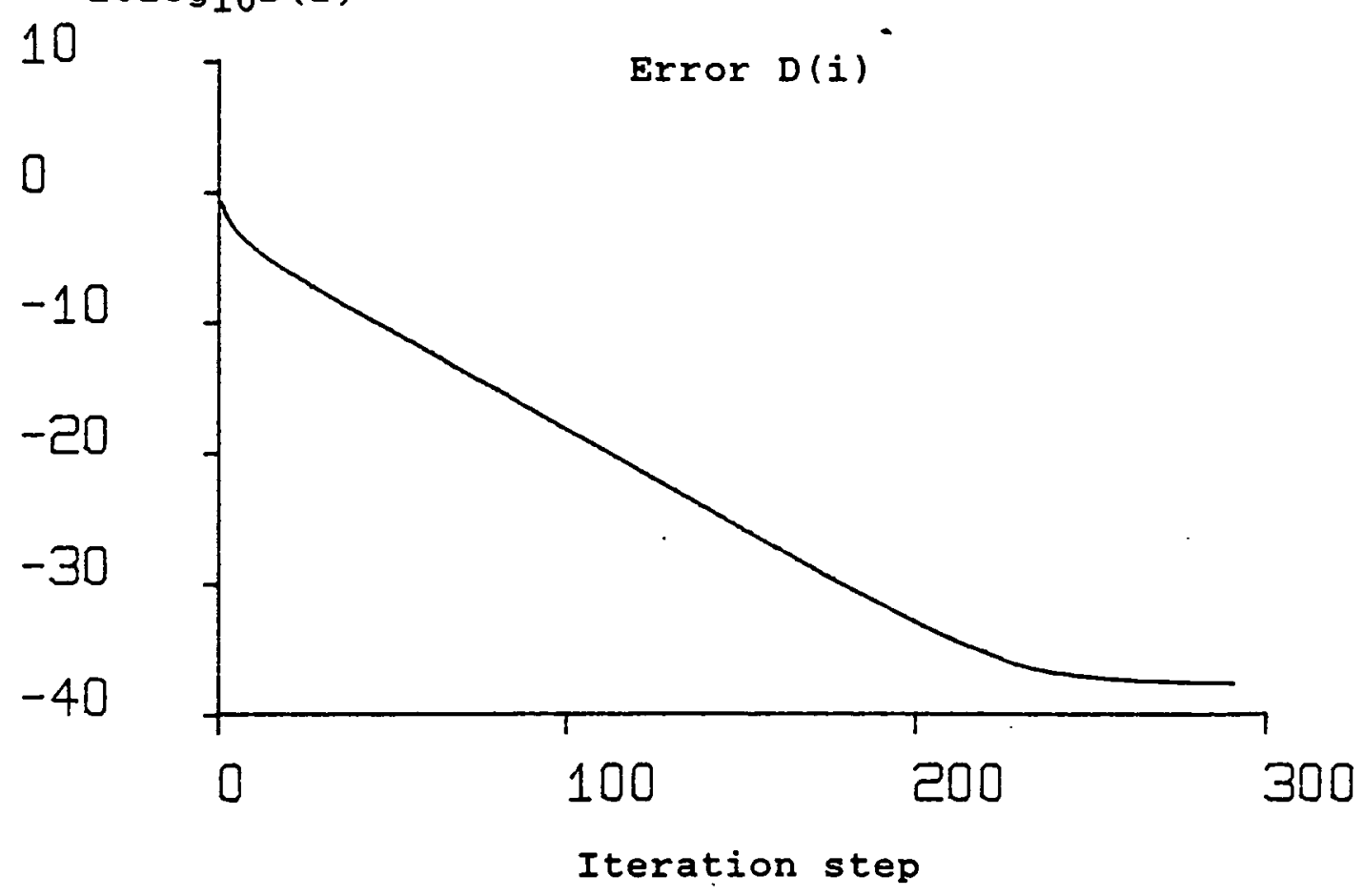

Figure 4.13 Convergence plots obtained using two calibration signals 
90 degrees and relative frequencies of 1.0 and 1.001 . Both of the signals had a power of $20 \mathrm{~dB}$ and an initial phase angle of zero degrees and the noise power was OdB. The covariance matrix was calculated by finding the expected value using the procedure described in Chapter 2 , thus allowing the errors caused by averaging over a finite number of snapshots to be eliminated.

The step size $\mu$ in the iteration was set to 0.005 . As anticipated, the cost function decreased at each step in the iteration. In this example, the convergence of $J(i)$ to a small value accurately reflects the behaviour of the unknown error $D(i)$.

Figure 4.14 shows the effects of errors in the bearings of the signals. The calibration algorithm was supplied with the same covariance matrix again but the bearings of the signals were specified incorrectly as being 20 and 87 degrees. Under these conditions, the improvement in the error $D(i)$ was extremely small. Notice, however, that the cost function $J(i)$ converged to a small value as before. Since $D(i)$ would not be available in a real application, the problem would remain undetected.

To demonstrate the advantage of using more than two signals, the process was repeated using three calibration signals with bearings of $-108,18$ and 90 degrees and 


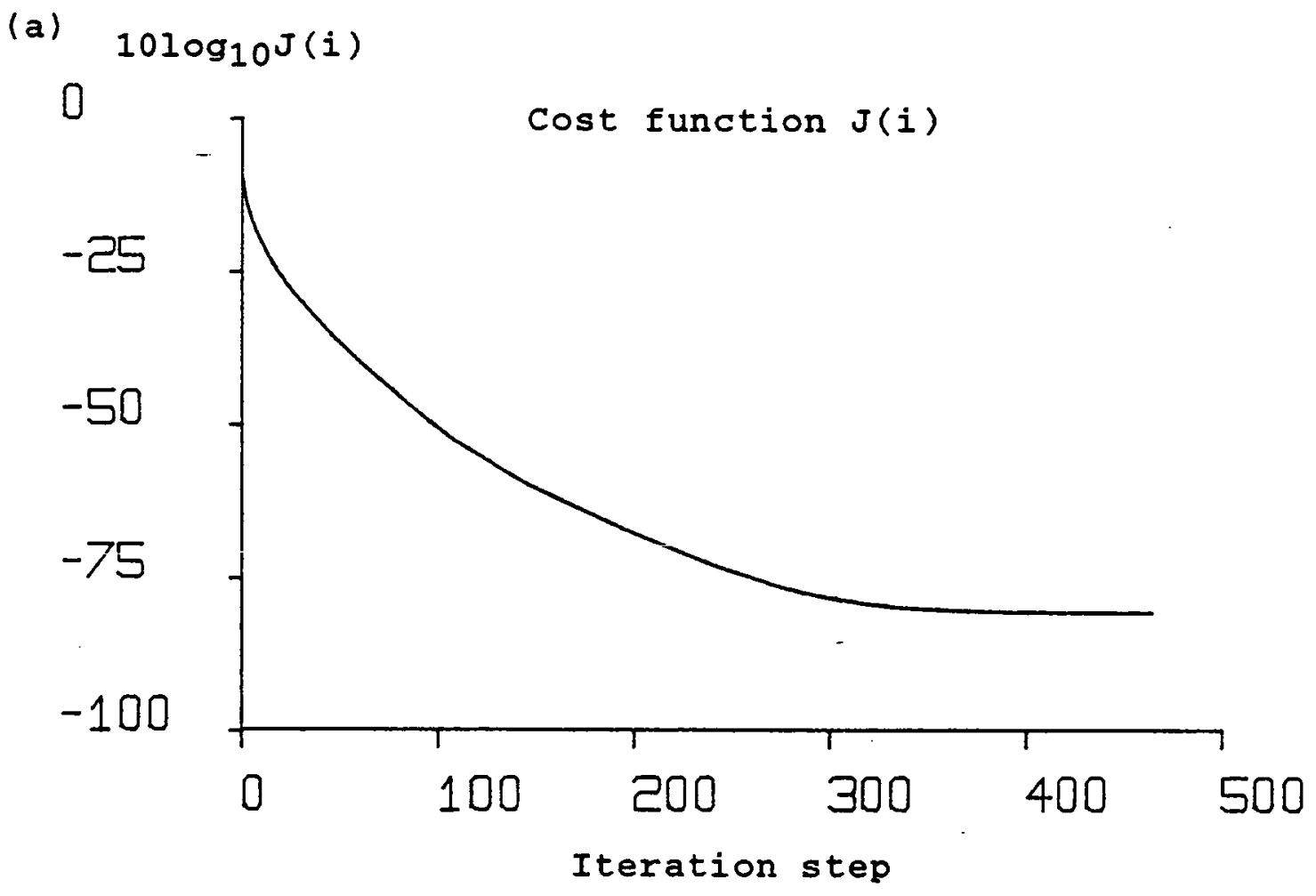

(b) $\operatorname{lol}_{10} \mathrm{D}(\mathrm{i})$

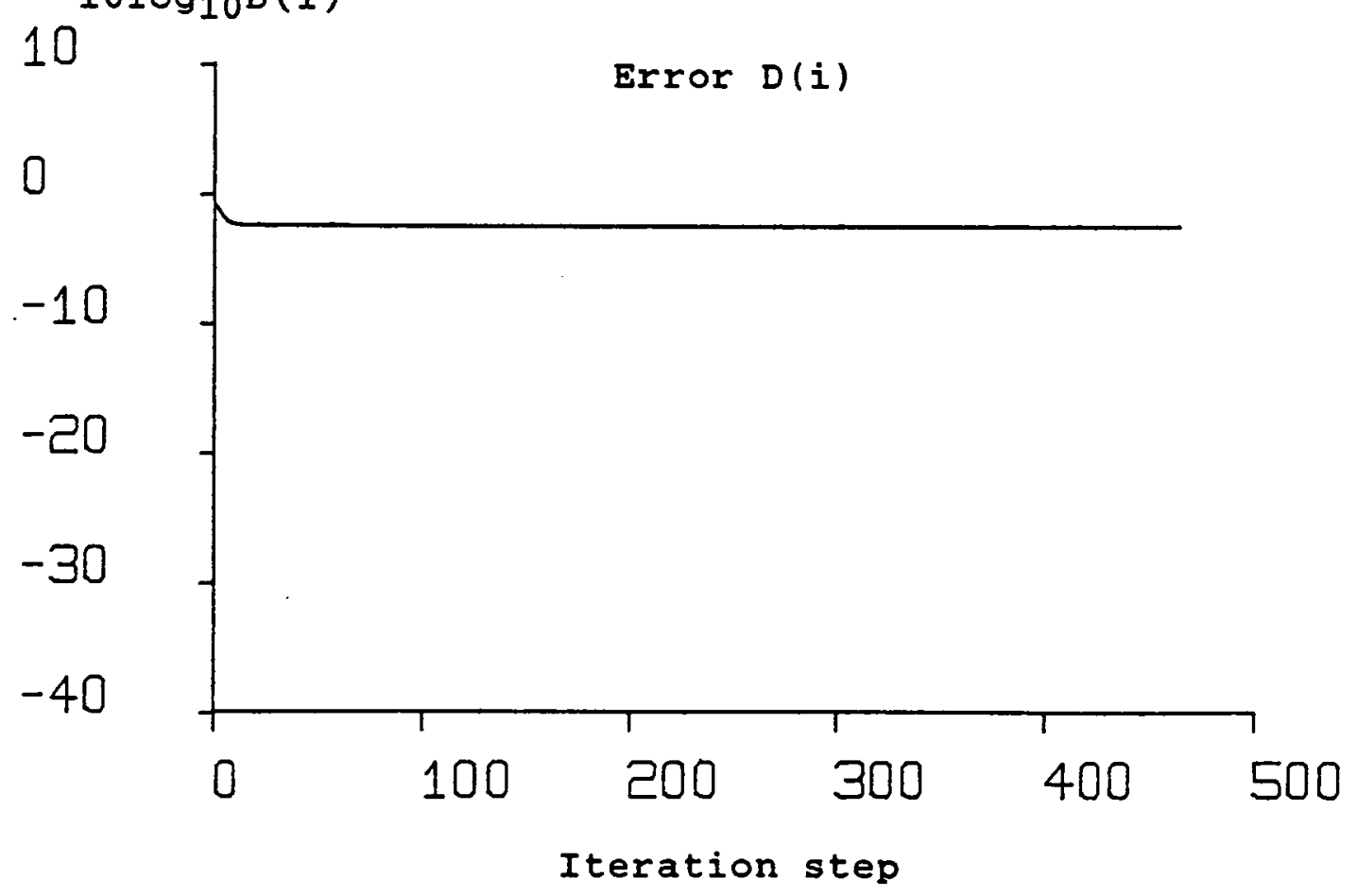

Figure 4.14 Convergence plots obtained using two calibration signals with inaccurate bearings 
relative frequencies of $0.999,1.0$, and 1.001

respectively. All of the signals had a power of $20 \mathrm{~dB}$ and an initial phase angle of zero degrees. The noise power was $O d B$ and the covariance matrix was calculated by taking the expected value as before.

Figures $4.15(a)$ and $4.15(b)$ show the behaviour of the cost function $J(i)$ and the error $D(i)$. Notice the shape of the $D(i)$ curve close to convergence. Having attained a minimum value, the estimate then gets slightly worse again before finally settling down. This effect is caused by inaccuracies in the computation of the eigenvectors of the covariance matrix. In principle, a MUSIC spectrum derived from the expected value of the covariance matrix would have peaks of infinite height for the reasons discussed in Chapter 2. In practice, however, the peaks are large but finite due to imperfections in the eigenvectors.

Since the calibration algorithm relies on the eigenvectors of the covariance matrix, it is also affected by such errors. In practice, the weight $n_{n m}(i)$ is not precisely equal to zero even when the estimated position of sensor $n$ is correct with respect to signal $\mathrm{m}$. The effect on the estimated sensor positions is small, although it is exaggerated somewhat by the logarithmic scale used in the diagram. It is worth pointing out that the problem is 


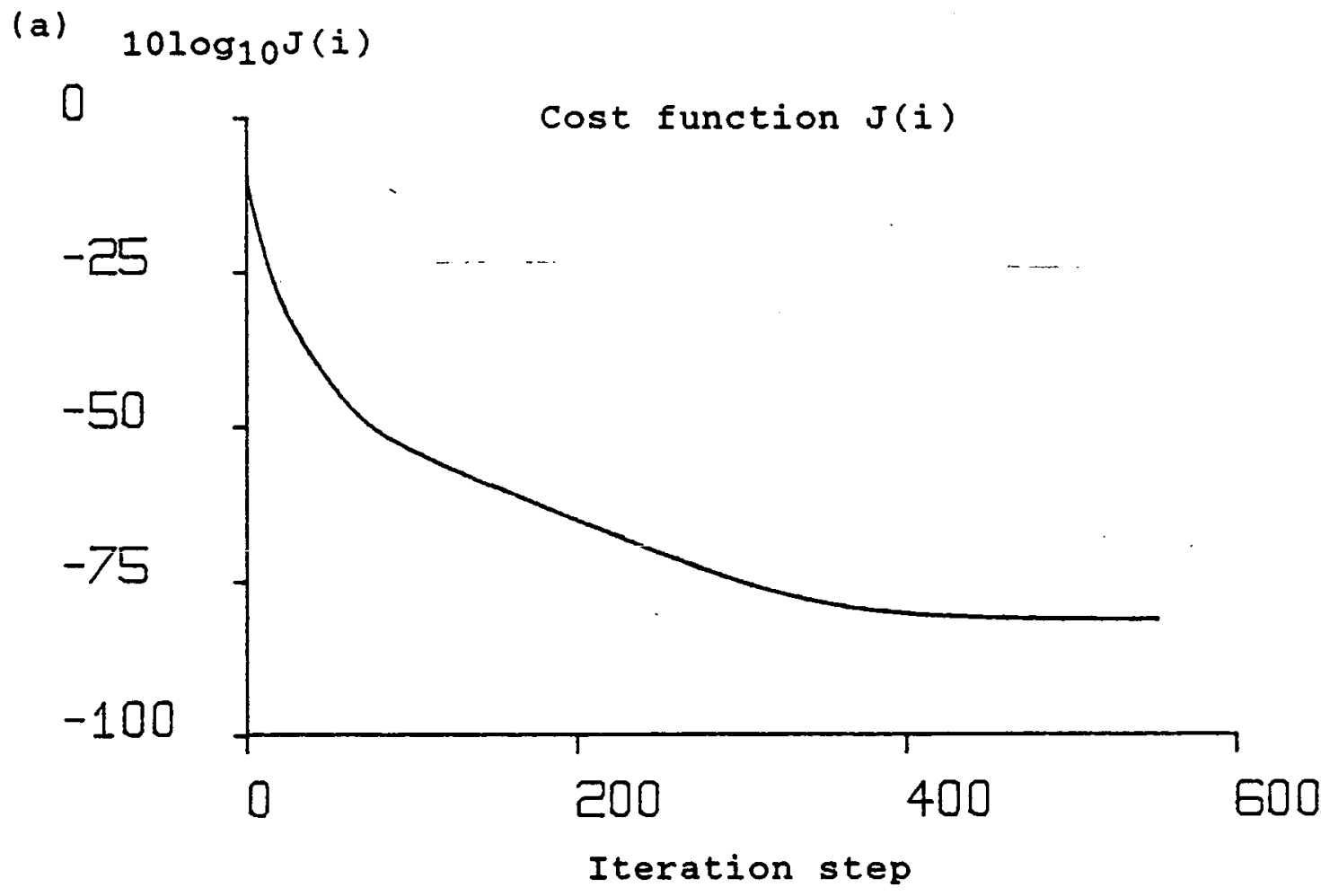

(b) $10 \log _{10} \mathrm{D}(\mathrm{i})$

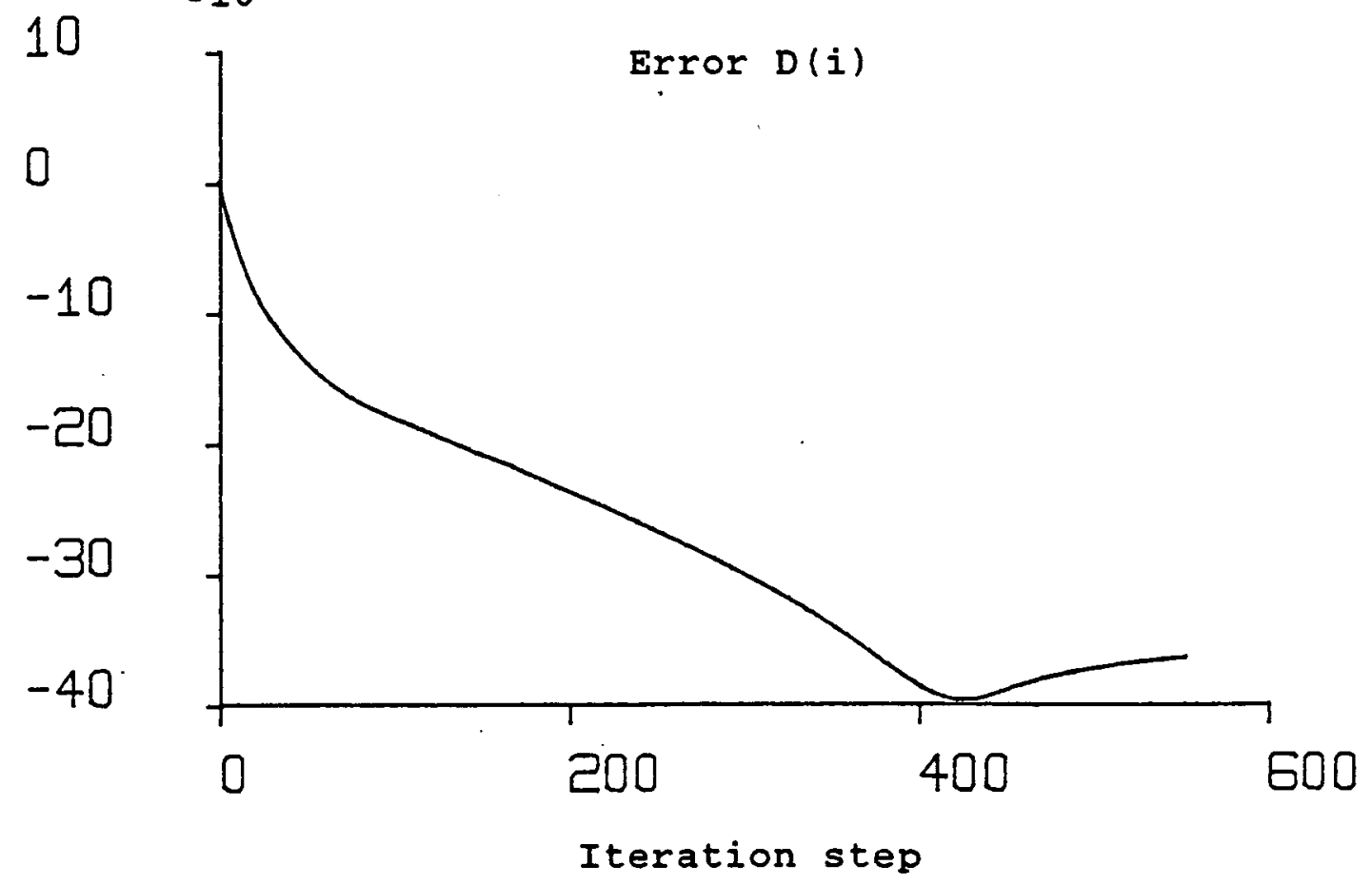

Figure 4.15 Convergence plots obtained using three calibration signals 
not related to the step size used in the iteration. If a smaller step size is adopted, the error $D(i)$ traces out the same curve over a larger number of steps.

Figure 4.16 shows the effects of errors in the bearings of the signals. The calibration algorithm was supplied with the same covariance matrix again but the bearings of the signals were specified incorrectly as being $-107,87$, and 20 degrees. As in the example where two signals were present, the improvement in the error $D(i)$ was very small.

However, with three calibration signals the cost function $J(i)$ also showed a small reduction, thus providing a warning that the calibration had not been successful. This happened because the iteration was unable to optimize the estimated sensor positions with respect to all three signals simultaneously. These results confirm that the cost function $J(i)$ provides a more faithful indication of the error $D(i)$ when the number of calibration signals is greater than two.

The next set of results explores the relationship between the quality of the covariance matrix $\underset{\sim}{\mathrm{R}}$ and the final values of $J(i)$ and $D(i)$ achieved by the iteration. As discussed earlier in the chapter, the effect of received noise is to add random errors to the off-diagonal elements of the covariance matrix. These errors are most 
(a) $10 \log _{10} \mathrm{~J}(i)$

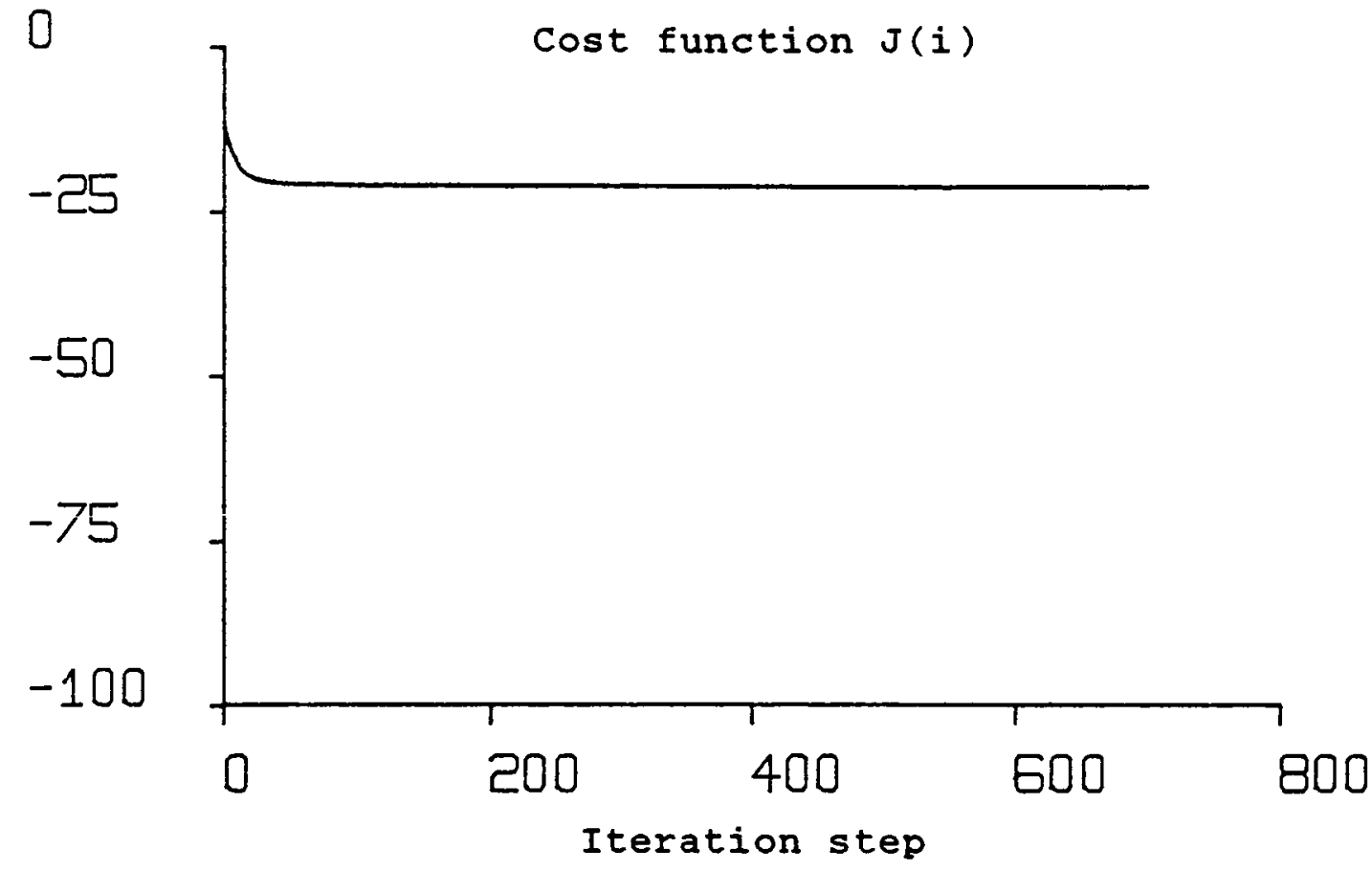

(b) $\log _{10} \mathrm{D}(\mathrm{i})$

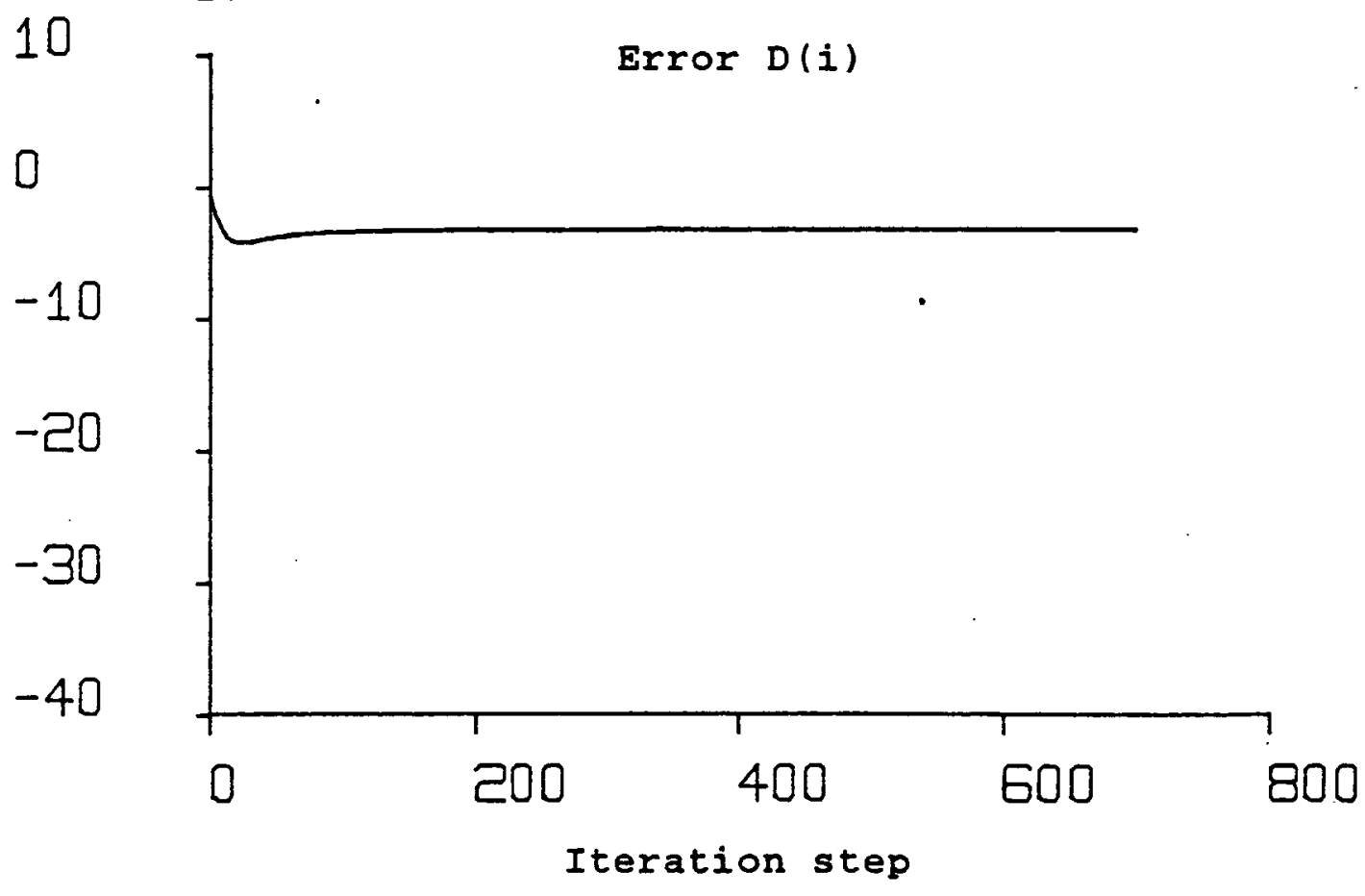

Figure 4.16 Convergence plots obtained using three calibration signals with inaccurate bearings 
pronounced when the signal to noise ratio is low and/or a small number of snapshots are used. The only way to eliminate such errors completely is to obtain the expected value of the covariance matrix, although this is clearly impossible in practice.

To investigate the effects of noise, the calibration algorithm was applied to a large number of covariañe matrices formed using different numbers of snapshots over a range of signal to noise ratios. There were three calibration signals with bearings of $-108,18$, and 90 degrees and relative frequencies of $0.999,1.0$, and 1.001 respectively. All of the signals had a power of $20 \mathrm{~dB}$ and an initial phase angle of zero degrees.

The signal to noise ratio was defined to be the ratio of the power of one of the signals to the noise power, meaning that a noise power of $20 \mathrm{~dB}$ corresponded to a signal to noise ratio of $\mathrm{OdB}$. The signal to noise ratio covered a range from $0 \mathrm{~dB}$ to $60 \mathrm{db}$ in steps of $10 \mathrm{~dB}$. At each signal to noise ratio, 20 covariance matrices were formed using 400 snapshots, another 20 using 1600 snapshots, and a further 20 using 6400 snapshots.

For each block of 20 covariance matrices, the calibration algorithm was applied to each matrix in turn and the averages of the final values of the cost function $J(i)$ and 
the error $D(i)$ were calculated. The calibration algorithm was also applied to the expected value of the covariance matrix at each signal to noise ratio. The step size $\mu$ was set to 0.005 and each calibration was terminated when the improvement in the cost function $|J(i+1)-J(i)|$ fell below $10^{-9}$.

The results are shown in Figure 4.17. As anticipated, the error $D(i)$ after calibration decreased when the signal to noise ratio or the number of snapshots was increased. When the expected value of the covariance matrix was used, there were no errors in the off-diagonal elements of the covariance matrix and the results were therefore independent of the signal to noise ratio.

The final set of results relates the finishing values of $J(i)$ and $D(i)$ to the accuracy to which the bearings of the calibration signals are stated. This was investigated by generating a single covariance matrix then applying the . calibration algorithm several times with random errors added to the bearings of the signals.

There were three calibration signals with bearings of $-108,18$, and 90 degrees and relative frequencies of $0.999,1.0$, and 1.001 respectively. All of the signals had a power of $20 \mathrm{~dB}$ and an initial phase angle of zero. degrees. So as to ensure that the results were not 
(a) $\log _{10} \mathrm{~J}(\mathrm{i})$

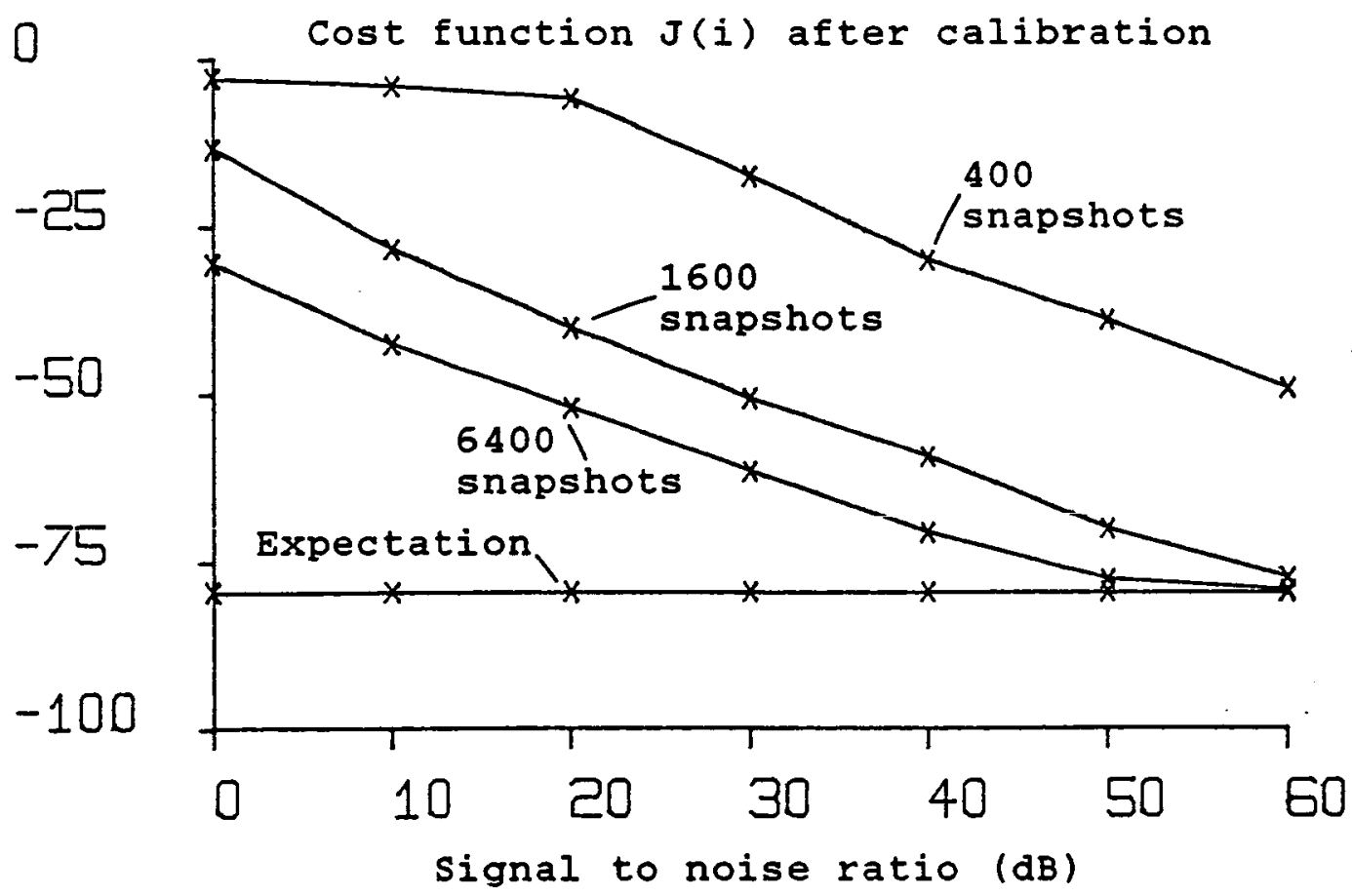

(b) $\log _{10} \mathrm{D}(\mathrm{i})$

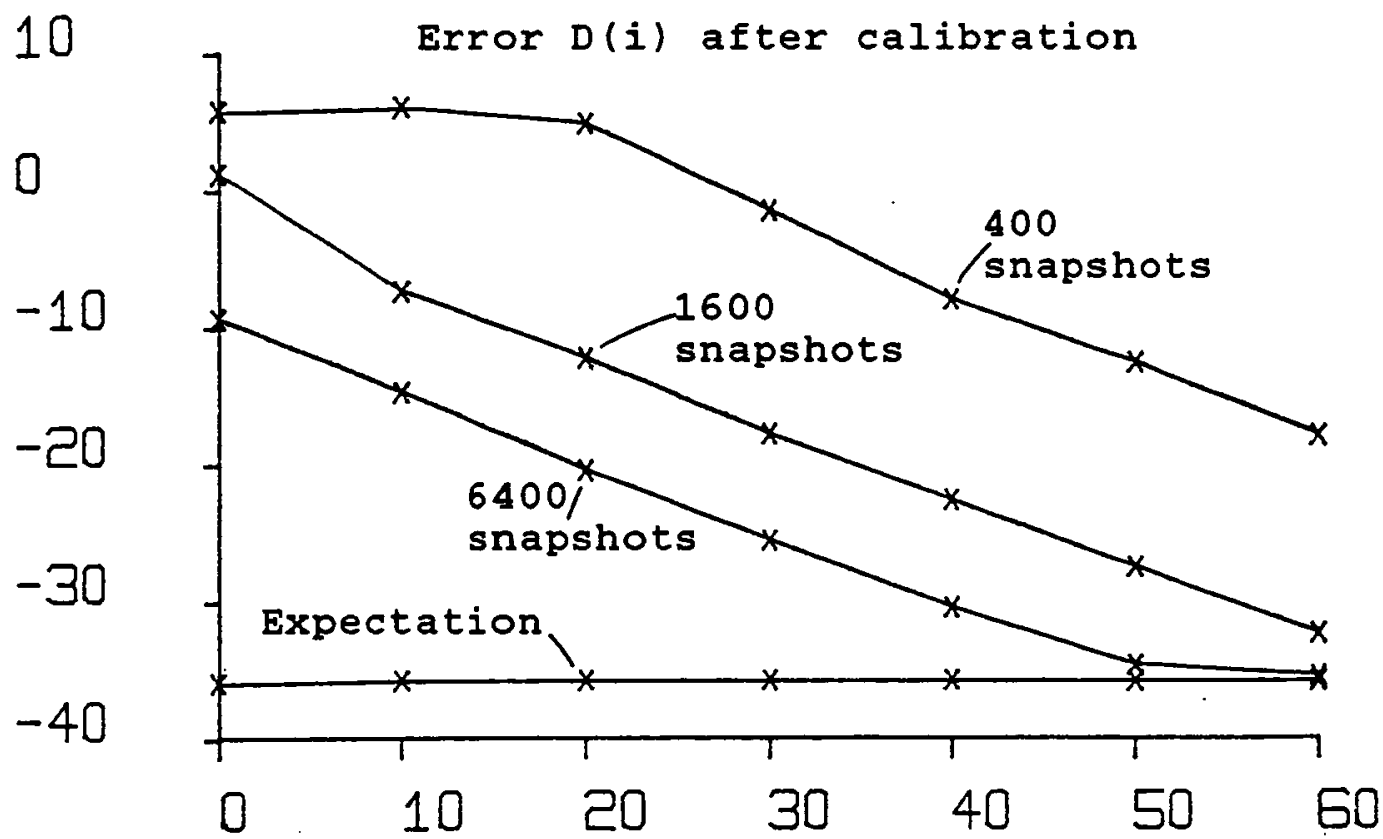

Signal to noise ratio (dB)

Figure 4.17 Influence of signal to noise ratio and number of snapshots 
influenced by errors in the off-diagonal elements, the covariance matrix was formed by taking the expected value. The noise power was odB.

Sets of incorrect signal bearings were generated by adding random numbers to the true bearings of the calibration signals. The random numbers had a normal distribution with a mean of zero. The variance assumed a number of values in the range from 0 to 0.5 degrees (squared). For each variance, 20 sets of incorrect signal bearings were generated and the calibration algorithm was applied to each one in turn. The final values of the cost function $J(i)$ and the error $D(i)$ were averaged over the 20 sets of bearings. The step size $\mu$ was set to 0.005 and each calibration was terminated when $|J(i-1)-J(i)|$ fell below $10^{-9}$.

The results are shown in Figure 4.18. Although the calibration algorithm only achieved dramatic reductions in $D(i)$ when the signal bearings were specified accurately, the error was reduced by approximately one order of magnitude across the entire range of variances. As the bearing spectra at the start of this section demonstrated, an improvement of this size is big enough to make a significant difference to the bearing spectrum. 
(a) $\operatorname{lol}_{10} \mathrm{~J}(\mathrm{i})$

0

$-25$

$-50$

$-75$

$-100$

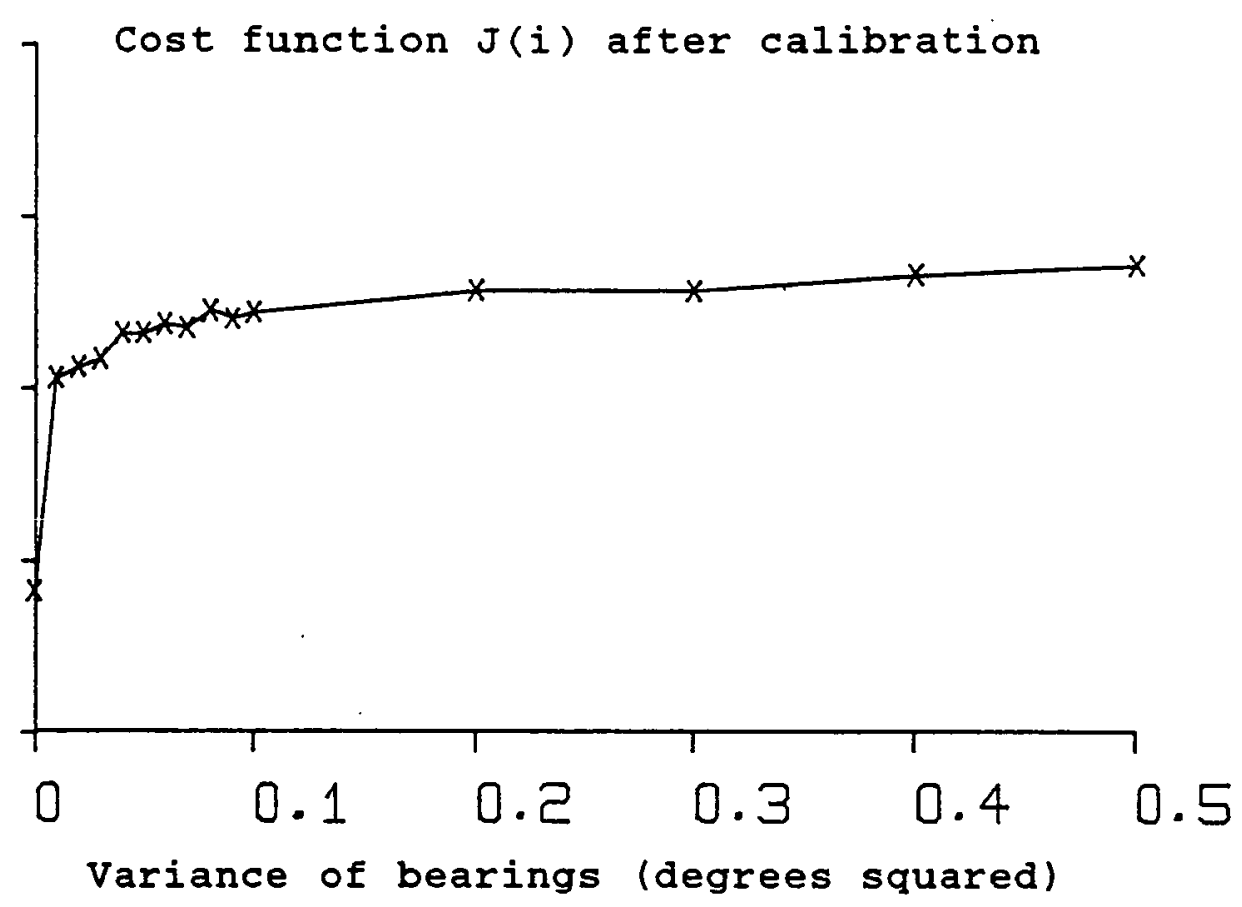

(b) $10 \log _{10} \mathrm{D}(\mathrm{i})$

10

Error D(i) after calibration
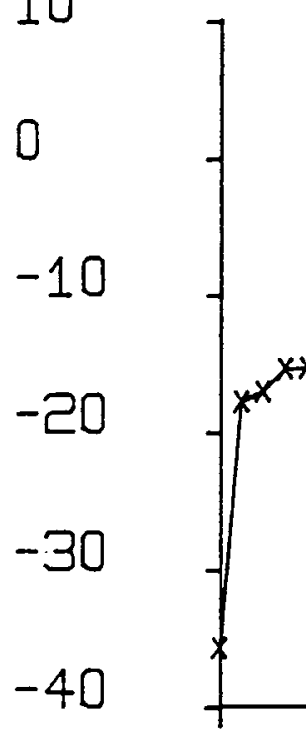


\subsection{Summary}

This chapter has developed a calibration algorithm to compensate for sensor positioning errors in direction finding arrays. The key point is that the degraded performance of an uncalibrated array is caused by the mismatch between the true sensor positions and the ones assumed by the bearing estimation algorithm. If the true sensor positions can be discovered, the performance of the system can be improved considerably.

The array calibration algorithm attempts to identify the true sensor positions by analyzing snapshots obtained while two or more signals with known bearings are being received. The positions are deduced through a process of triangulation.

The algorithm takes the form of an iteration in which a set of estimated sensor positions is steered towards the (unknown) true positions, the intended sensor positions being the initial estimates. This goal is achieved by minimizing a cost function which is based on the signal subspace concepts presented in Chapter 2 .

The improvements obtained using the calibration algorithm were demonstrated using computer simulations. 


\section{CHAPTER 5 CONCLUSIONS}

\subsection{Material Covered}

This thesis has examined some of the signal processing algorithms used for bearing estimation with particular attention to the MUSIC technique. A derivation of MUSIC from first principles produced an understanding of the signal subspace approach to bearing estimation and introduced many of the concepts required for the original research.

This was followed by an analysis of the effects of sensor positioning errors on the bearing spectrum. A statistical approach produced theoretical results, most of which were independent of the particular array geometry being used. These results were confirmed using computer simulations.

Having identified the effects of sensor positioning errors, an array calibration algorithm was developed to reduce the sensitivity of the bearing estimation process to such errors. The algorithm estimated the true sensor positions by exploiting two or more signals with known bearings. Computer simulations demonstrated the 
improvements obtained after calibration.

\subsection{Specific Achievements}

Recalling the aims of this work stated in Chapter 1 , the two main objectives were to characterize the sensitivity of bearing estimation algorithms to sensor positioning errors and to reduce this sensitivity through array calibration. These two areas were addressed in Chapters 3 and 4 respectively.

In Chapter 3 , the conventional beamforming, minimum energy, and MUSIC bearing estimation techniques were compared in the presence of small sensor positioning errors. With only one signal present, all of these methods are normally unbiased estimators of signal bearing and the analysis demonstrated that they remain unbiased in the presence of sensor positioning errors with the assumed statistics.

However, the variability of the bearing indicated by the spectrum increased with the size of the sensor positioning errors and it was shown that the variance of the bearing was directly proportional to the variance of the sensor positions. If the array geometry was taken into account, 
the constant of proportionality could be calculated for a particular bearing. It would be straightforward to average this over a range of bearings in order to produce a figure of merit for a particular array. The constant was independent of the bearing estimation technique being used.

Further results provided insight into the behaviour of the mean and variance of the peak height. As the variance of the sensor positions increased, the mean peak height decreased while the variance of the peak height increased. Although the theoretical results described the height of the spectrum at the correct bearing rather than the true peak height, they provided theoretical Iimits for the peak height. Results for all three bearing estimation techniques were produced.

Computer simulations verified the theoretical curves which had been predicted. The simulations were also used in areas not covered by theoretical work, such as demonstrating the decreased resolution of the MUSIC method in the presence of sensor positioning errors.

In Chapter 4, an array calibration algorithm to compensate for sensor positioning errors was developed. It was shown that when two or more signals with known bearings are present, the true sensor positions are observable from 
the array snapshots provided that each sensor is within half a wavelength of its intended position.

An iterative scheme was used to guide a set of estimated sensor positions towards the true ones, taking the theoretical sensor positions as initial values. Computer simulations demonstrated the effectiveness of the technique in reducing the errors in the estimates.

The errors remaining after calibration were summed over all of the sensors and expressed on a logarithmic scale. It was found that the overall error decreased as the signal to noise ratio or the number of snapshots was increased.

Although the algorithm relied on accurate signal bearings, the effects of incorrect bearings were considered. Errors in the bearings caused the sensor positions to be estimated incorrectly and in extreme cases the estimated positions were worse than the intended ones.

However, the behaviour of the cost function provided a warning of this problem if more than two signals were present. Computer simulations showed the tolerance required for the signal bearings in a particular scenario.

The author has presented two conference papers on the 
calibration algorithm [103], [104].

\subsection{Limitations}

The results obtained in Chapter 3 for the spectrum height in the minimum energy and MUSIC methods are somerhat awkward in that they apply to the reciprocal of the function used in forming the spectrum. It would, however, have been extremely difficult to take the analysis any further without resorting to an inequality as $E[1 / X] \neq 1 / E[X]$. Since the results are precise as $f a r$ as they go, the mental adjustment required to think in terms of the reciprocal seems worthwhile.

An obvious limitation of the work described in Chapter 4 is the reliance on signals with known bearings. Indeed, this will render the method impractical in some situations.

It is worth noting, however, that one of the radio direction finding systems marketed by the company sponsoring this work incorporates a very rudimentary calibration mechanism which was requested by the customer. A portable transmitter is moved around to provide calibration signals from different directions. It appears that users are willing to accept such 
procedures if they offer an improvement in performance.

\subsection{Suggestions for Further Research}

In Chapter 4, the possibility of implementing the calibration algorithm using matrix inversion was mentioned briefly. It might be worthwhile to investigate this approach and compare it with the iterative scheme adopted.

As noted in Chapter 1, a number of papers describing other calibration algorithms were discovered while this thesis was being prepared and these techniques should be compared with the method described in Chapter 4. Some authors have described algorithms which are claimed to operate on calibration signals with unknown bearings, thus making the process more practical.

It would be interesting to extend the analysis presented in Chapter 3 to include theoretical results for the peak height as well as the height of the spectrum at the correct bearing. This would require a more sophisticated approach than the one adopted in Chapter 3 since the peak height depends on the incorrect bearing, which is itself a random variable. 
REFERENCES

[1] S.Haykin, Ed.. Array Signal Processing. New Jersey: Prentice-Hall, 1985.

[2] P.J.Baldwin, "An adaptive array for radar applications," Electronics Research Council Symposium on Adaptive Antenna Array Signal Processing, vol.1, May 1979.

[3] C.Li and H.Wang, "Active high-resolution multiple-target direction finding," ICASSP 1988 , pp. 2837-2840.

[4] J.Bodonyi and T.W.Pegram, "H.F. ground-wave radar; theory and practice," Fourth Int. Conf. on HF Radio Systems and Techniques, IEE Conf. Publication Number 284, pp.241-245, 1988.

[5] R.H.Perry and P.A.Hamshere, "An experimental HF ground wave radar," Fourth Int. Conf. on HF Radio Systems and Techniques, IEE Conf. Publication Number 284, pp.246-249, 1988.

[6] J.R.Eastwell, "Direction finding using a wide aperture circular array with minimum antenna elements," Fourth Int. Conf. on HF Radio Systems and Techniques, IEE Conf. Publication Number 284 , pp. 231-235, 1988 .

[7] D.E.N.Davies, N.Karavassilis, and B.S.Collins, "An active circular HF array," Third Int. Conf. on HF Communication Systems and Techniques, IEE Conf. Publication Number 245, pp.99-101, 1985.

[8] R.A.Monzingo and T.W.Miller, Introduction to Adaptive Arrays. New York: John Wiley \& Sons, 1980 .

[9] W.C.Knight, R.G.Pridham, and S.M.Kay, "Digital signal processing for sonar," Proc. IEEE, vol.69, pp.1451-1506, Nov.1981.

[10] B.Sheleg, "A matrix-fed circular array for continuous scanning," PrOC. IEEE, vol.56, pp.2016-2027, Nov.1968. 
[11] R.O.Schmidt, "New mathematical tools in direction finding and spectral analysis," SPIE Sixth Conference on Real Time Signal Processing, pp. 7-19, 1983 .

[12] S.R.DeGraaf and D.H.Johnson, "Capability of array processing algorithms to estimate source bearings," IEEE Trans. Acoustics, Speech, Signal Process., vol.ASSP-33, pp.1368-1379, Dec.1985.

[13] O.L.Frost, "Adaptive interference and multipath rejection in linear direction-finding arrays," ICASSP 1986, pp.2499-2502.

[1A] T.Shan and T.Kailath, "Adaptive beamforming for coherent signals and interference," IEEE Trans. Acoustics, Speech, Signal Process., vol.ASSP-33, pp.527-536, Jun.1985.

[15] W.P.Ballance and A.G.Jaffer, "Direction finding in the presence of fully correlated specular multipath," ICASSP 1988, pp.2849-2852.

[16] D.H.Johnson, "The application of spectral estimation methods to bearing estimation problems," Proc. IEEE, vol.70, pp.1018-1028, Sep.1982.

[17] S.L.Marple, Digital spectral Analysis. New Jersey: Prentice-Hall, 1987.

[18] S.M.Kay and S.L.Marple, "Spectrum analysis - a modern perspective," Proc. IEEE, vol.69, pp.1380-1416, Nov.1981.

[19] J.A.Cadzow, "High-resolution spectral estimation: an assessment," Trends and Perspectives in signal Processing, vol.7, pp.16-19, Jun.1983.

[20] J.Makhoul, "Linear prediction: a tutorial review," Proc. IEEE, vol.63, pp.561-580, Apr.1975.

[21] R.T.Lacoss, "Data adaptive spectral analysis methods," Geophysics, vol.36, pp.661-675, Aug. 1971 .

[22] R.0.Schmidt, "Multiple emitter location and signal parameter estimation," Proc. RADC Spectrum Estimation Workshop, pp.243-258, 1979.

[23] B.Friedlander and A.J.Weiss, "Eigenstructure methods for direction finding with sensor gain and phase uncertainties," ICASSP 1988, 
pp. 2681-2684.

[24] S.R.Degraaf and D.H.Johson, "Capability of array processing algorithms to estimate source bearings," ICASSP 1983, pp.344-347.

[25] R.E.Hernaman, "High resolution adaptive array processing - a review of algorithms and performance," IEE Collogium on Digital Processing in Sonar, pp.3/1-3/6, 1983 .

[26] D.H.Johnson and S.R.DeGraaf, "Improving the resolution of bearing in passive sonar arrays by eigenvalue analysis," IEEE Trans. Acoustics, Speech, Signal Process., vol.ASSP-30, pp.638-647, Aug. 1982 .

[27] R.S.Walker, "Bearing accuracy and resolution bounds of high-resolution beamformers," ICASSP 1985, pp.1784-1787.

[28] J.E.Evans, "Aperture sampling techniques for precision direction finding," IEEE Trans. Aerosp. Electron. Syst., Corresp., vol.AES-15, pp. 891-895, Nov.1979.

[29] E.R.Ferrara and T.M.Parks, "Direction finding with an array of antennas having diverse polarizations," IEEE Trans. Antennas Propagat., vol.AP-31, pp.231-236, Mar.1983.

[30] W.F.Gabriel, "Spectral analysis and adaptive array superresolution techniques," Proc. IEEE, vol.68, pp.654-666, Jun.1980.

[31] D.R.Farrier, "Maximum likelihood, maximum entropy and their role in signal detection and identification," IEE COIlogium on Signal Processing Arrays, pp.5/1-5/4, 1984 .

[32] A.T.Parsons, "A theoretical comparison of maximum entropy and maximum likelihood detection performances," IEE Colloqium on Signal Processing Arrays, pp.4/1-4/6, 1984.

[33] J.Capon, R.J.Greenfield, and R.J.Rolker, "Multidimensional maximum-likelihood processing of a large aperture seismic array," Proc. IEEE, vol.55, pp.192-211, Feb.1967.

[34] U.Sandkuhler and J.F.Bohme, "Accuracy of maximum likelihood estimates for array processing," ICASSP 1987, pp.2015-2018. 
[35] D.N.Swingler, R.S.Walker, and G.B.Lewis, "Linear prediction for aperture extrapolation in line array beamforming," ICASSP 1986, pp.2519-2522.

[36] S.Haykin, T.Greenlay, and J.Litva, "Performance evaluation of the modified FBLP method for angle of arrival estimation using real radar multipath data," Proc. IEE, vol.132, pt.F, pp.159-174, Jun. 1985 .

[37] A.Abo-Zena and E.L.Church, "Multiple target detection - optimum resolution," ICASSP 1986. pp. 2515-2518.

[38j S.S.Reddi, "Multiple source location - a digital approach," IEEE Trans. Aerosp. Electron. Syst., vol.AES-15, pp.95-105, Jan.1979.

[39] D.H.Johnson, "Properties of eigenanalysis methods for bearing estimation algorithms," ICASSP 1985. pp. 552-555.

[40] B.W.Dahanayake and K.M.Wong, "Proper orthogonal projection - multiple signal classification (POP-MUSIC)," ICASSP 1986, pp.2491-2494.

[41] M.Wax and T.Kailath, "Extending the threshold of the eigenstructure methods," ICASSP 1985, pp. 556-559.

[42] L.Zou and L.Yin, "Spatio-temporal spectral analysis by SVD of signal matrix," ICASSP 1987, pp. 2332-2335.

[43] B.W. Dahanayake, "A new method of array processing," ICASSP.1987, pp.2300-2303.

[44] J.Capon, "High-resolution frequency-wavenumber spectral analysis," Proc. IEEE, vol.57, pp.1408-1418, Aug.1969.

[45] T.P.Bronez and J.A.Cadzow, "An algebraic approach to superresolution array processing," IEEE Trans. Aerosp. Electron. Syst., vol.AES-19, pp.123-133, Jan.1983.

[46] D.W.Tufts, I.Kirsteins, and R.Kumaresan, "High resolution detection and estimation," SPIE Sixth Conference on Real Time Signal Processing, pp. 20-31, 1983 .

[47] G.Bienvenu and L.Kopp, "Adaptivity to background noise spatial coherence for high resolution 
passive methods," ICASSP 1980, pp.307-310.

[48] S.R.Hui, B.L.Lim, and Y.C.Lim, "An adaptive recursive array processing algorithm for resolving closely spaced signals with 10 WN $S$ and widely disparate signal power," ICASSP 1988. pp. 2897-2900.

[49] J.C.Chung and A.W.Robertson, "Resolution of coherent signals using random array," ICASSP 1988, pp. 2678-2680.

[50] A.Paulraj and T.Kailath, "On beamforming in the presence of multipath," ICASSP 1985, pp.564-567.

[51] T.Shan, M.Wax, and T.Kailath, "On spatial smoothing for direction of arrival estimation of coherent signals," IEEE Trans. Acoustics, Speech, Signal Process., vol.ASSP-33, pp.806-811, Aug. 1985 .

[52] M.P.Moody, "Resolution of coherent sources incident on a circular array," Proc. IEEE, vol.68, pp.276-277, Feb.1980.

[53] E.R.Miller, "Comments on resolution of coherent sources incident on a circular array," Proc. IEEE, vol.69, pp.122-123, Jan.1981.

[54] H.Clergeot, A.Ouamri, and S.Tressens, "High resolution spectral methods for spatial discrimination of closely spaced correlated sources," ICASSP 1985, pp.560-563.

[55] M.Wax, T.Shan, and T.Kailath, "Spatio-temporal spectral analysis by eigenstructure methods," IEEE Trans. Acoustics, Speech, Signal Process., vol.ASSP-32, pp.817-827, Aug.1984.

[56] C.Yeh, J.Lee, and Y.Chen, "Estimating two-dimensional angles of arrival in coherent source environment," ICASSP 1988, pp.2909-2912.

[57] Q.Yin and L.Zou, "Separately estimating the frequency wavenumber of coherent sources by signal smoothing technique," ICASSP 1988, pp.2905-2908.

[58] A.G.Jaffer, "Maximum likelihood direction finding of stochastic sources: a separable solution," ICASSP 1988, pp.2893-2896. 
[59] J.A.Cadzow, Y.S.Kim, W.Ma, and D.C.Shiue, "Resolution of spatially close broadband coherent sources incident on a general array," ICASSP 1988, pp.2662-2665.

[60] S.U.Pillai, F.Haber, and Y.Bar-Ness, "A new approach to array geometry for improved spatial spectrum estimation," ICASSP 1985, pp.1816-1819.

[61] Y.Lee and S.U.Pillai, "An algorithm for optimum placement of sensor elements," ICASSP 1988, pp. 2674-2677.

[62] A.E.Cetin and R.Ansari, "A procedure for antenna array pattern synthesis," ICASSP 1987. pp. 2308-2311.

[63] R.Rumaresan and A.Shaw, "High resolution bearing estimation without eigen decomposition," ICASSP 1985, pp.576-579.

[64] E.R.L.Hung and R.M.Turner, "A fast beamforming algorithm for large arrays," IEEE Trans. Aerosp. Electron. Syst., vol.AES-19, pp.598-607, Jul.1983.

[65] S.H.Nawab, F.U.Dowla, and R.T.Lacoss, "Direction determination of wideband signals," IEEE Trans. Acoustics, Speech, Signal Process., vol.Assp-33, pp.1114-1122, Oct.1985.

[66] R.Roy, A.Paulraj, and T.Kailath, "Comparative performance of ESPRIT and MUSIC for direction of arrival estimation," ICASSP 1987, pp.2344.2347.

[67] R.RoY, A.Paulraj, and T.Kailath, "ESPRIT - a subspace rotation approach to estimation of parameters of cisoids in noise," IEEE Trans. Acoustics, Speech, Signal Process., vol.ASSP-34, pp.1340-1342, Oct.1986.

[68] Y.Chen, J.Lee, and C.Yeh, "Calibration free bearing estimation for arrays with randomly perturbed sensor locations," ICASSP 1988, pp. 2917-2920.

[69] A.J.Weiss and B.Friedlander, "Array shape calibration using sources in unknown locations a maximum likelihood approach," ICASSP 1988, pp. 2670-2673.

[70] T.Tollefsen and A.Nehorai, "Adaptive calibration of randomly perturbed sensor arrays," ICASSP 1988, pp.2646-2649. 
[71] Y.Rockah and P.M.Schultheiss, "Array shape calibration using sources in unknown locations part 1: far-field sources," IEEE Trans.

Acoustics, Speech, Signal Process., vol.Assp-35, pp.286-299, Mar.1987.

[72] Y.Rockah and P.M.Schultheiss, "Array shape calibration using sources in unknown locations part 2: near-field sources and estimator implementation," IEEE Trans. Acoustics, Speech, Signal Process., vol.ASSP-35, pp.724-735, Jun. 1987 .

[73] N.K.Jablon, "Effect of element errors on half-power beamwidth of the Capon adaptive beamformer," IEEE Trans. Circuits, Systems, vol.CAS-34, pp.743-752, Jul.1987.

[74] C.N.Dorny, "A self-survey technique for self-cohering of antenna systems," IEEE Trans. Antennas Propagat., vol.AP-26, pp.877-881, Nov. 1978 .

[75] J.T.Lo and S.L.Marple, "Eigenstructure methods for array sensor localization," ICASSP 1987, pp. 2260-2263.

[76] C.H.Knapp, "Signal detectors for arrays with randomly perturbed sensor locations," ICASSP 1987, pp.2336-2339.

[77] C.N.Dorny and B.S.Meagher, "Cohering of an experimental nonrigid array by self-survey," IEEE Trans. Antennas Propagat., vol.AP-28, pp.902-904, Nov. 1980 .

[78] M.J.Hinich and W.Rule, "Bearing estimation using a large towed array," J. Acoust. Soc. Amer., vol.58, pp.1023-1029, 1975.

[79] M.J.Hinich and W.Rule, "Bearing estimation using a perturbed linear array," $J$. Acoust. Soc. Amer., vol.61, pp.1540-1544, 1977 .

[80] G.C.Carter, "Passive ranging errors due to hydrophone position uncertainty," U.S. Navy, $J$. Underwater Acoust., vol.29, pp.79-89, 1979.

[81] P.M.Schultheiss and J.P.Ianniello, "Optimum range and bearing estimation with randomly perturbed arrays," J. Acoust. Soc. Amer., vol.68, pp.167-173, 1980. 
[82] S.Rotheram, "Ground-wave propagation - part 1: theory for short distances," Proc. IEE, vol.128, pt.F, pp.275-284, Oct.1981.

[83] S.Rotheram, "Ground-wave propagation - part 2: theory for medium and long distances," Proc. IEE, vol.128, pt.F, pp.285-295, oct.1981.

[84] J.E.Hudson, Adaptive Array Principles. Stevenage, UR: Peter Peregrinus, 1981.

[85] G.Strang, Linear Algebra and its Applications. New York: Academic Press, 1980.

[86] F.Ayres, Theory and Problems of Matrices. New York: McGraw-Hill, 1974.

[87] G.Bienvenu and L.Ropp, "Optimality of high resolution array processing using the eigensystem approach," IEEE Trans. Acoustics, Speech, Signal Process., vol.ASSP-31, pp.1235-1247, Oct.1983.

[88] M.Wax and I.Ziskind, "Detection of fully correlated signals by the MDL principle," ICASSP 1988, pp.2777-2780.

[89] B.W.Dahanayake and R.M.Wong, "Detection: a new approach," ICASSP 1988, pp.2773-2776.

[90] K.M.Wong, Q.T.zhang, J.P.Reilly, and P.Yip, "A new criterion for the determination of the number of signals in high-resolution array processing," ICASSP 1988, pp.2769-2772.

[91] D.B.Williams and D.H.Johnson, "Modifying the sphericity test for improved source detection with narrowband passive arrays," ICASSP 1987, pp. 2272-2275.

[92] F.Rozin and F.Nakajima, "The order determination problem for linear time-varying AR models," IEEE Trans. Automat. Contr., vol.AC-25, pp.250-257, Apr. 1980 .

[93] H.Tong, "Autoregressive model fitting with noisy data by Akaike's information criterion," IEEE Trans. Informa. Theory, pp.476-479, Jul.1975.

[94] H.Tong, "More on autoregressive model fitting with noisy data by Akaike's information criterion," IEEE Trans. Informa. Theory, pp.409-410, May.1977. 
[95] D.H.Brandwood, "A complex gradient operator and its application in adaptive array theory," Proc. IEE, vol.130, pts.F and H, pp.11-16, Feb.1983.

[96] Sun Microsystems, UNIX Interface Reference Manual. Mountain View, California: Sun

Microsystems, Part Number 800-1303-04, 1986.

[97] P.L.Meyer, Introductory Probability and Statistics. Reading, Massachusetts: Addison-Wesley, 1973.

[98] F.James, "Monte carlo theory and practice," CERN Data Handling Division, submitted to Reports on Progress in Physics, Feb.1980.

[99] L.Kaufman, "Algorithm 496 - the LZ algorithm to solve the generalized eigenvalue problem for complex matrices," ACM Trans. Math. Software, vol.1, pp.271-281, Sep.1975.

[100] E.Kreyszig, Advanced Engineering Mathematics. New York: John Wiley \& Sons, 1983.

[101] M.Abramowitz and I.A.Stegun, Eds., Handbook of Mathematical Functions. New York: Dover Publications, 1972 .

[102] B.Widrow and S.D.Stearns, Adaptive Signal Processing. Englewood Cliffs: Prentice-Hall, 1985.

[103] L.P.H.K.Seymour, C.F.N.Cowan, and P.M.Grant, "A new calibration algorithm to detect sensor positioning errors in direction finding arrays," IEE Colloquium on Digital Signal Processing, IEE Digest Number 1987/14, pp.10/1-10/4, 1987.

[104] L.P.H.K.Seymour, C.F.N.Cowan, and P.M.Grant, "Bearing estimation in the presence of sensor positioning errors," ICASSP 1987, pp.2264-2267. 


\section{RELEVANT PUBLICATIONS}

This section contains copies of two conference papers which the author presented during 1987.

The first paper, "A new calibration algorithm to detect sensor positioning errors in direction finding arrays" [103], was given at the IEE Colloquium on Digital Signal Processing on 26th January 1987 (IEE Digest Number $1987 / 14)$.

The second paper, "Bearing estimation in the presence of sensor positioning errors" [104], was presented at the 1987 International Conference on Acoustics, Speech, and Signal Processing (ICASSP). 


\title{
A NEW CALIBRATION ALGORITHM TO DETECT SENSOR POSITIONING ERRORS IN DIRECTION FINDING ARRAYS
}

\author{
L.P.H.K.Seymour, C.F.N.Cowan, and P.M.Grant
}

\section{Introduction}

In bearing estimation, the outputs from a set of sensors are analysed to determine the bearings of signals arriving at the array $[1,2,3]$. The positions of the sensors are normally assumed to be known precisely. However, if there are errors in the positions then these assumptions are false and the bearing estimation is less accurate. Brandwood [4] presents examples of bearing spectra obtained using incorrect positions.

This paper compares the effects of sensor positioning errors on the conventional beamforming, Minimum Energy, and MUSIC bearing estimation techniques. High resolution methods, such as MUSIC, are shown to be the most sensitive. A new calibration algorithm to compensate for such errors is outlined. The new method is an extension of MUSIC in which transmissions with known bearings are analysed to determine the true sensor positions. These are subsequently used by a conventional bearing estimation algorithm to determine the angles of arrival of unknown transmissions. Simulation results illustrate the effects of sensor positioning errors and the improvements obtained using the new algorithm

\section{Comparison of Bearing Estimation Techniques}

The simulation results described below were obtained using an array consisting of five sensors evenly spaced around a circle of radius $0.5 \lambda$ with a sixth sensor at the centre. These "theoretical" sensor positions describe the intended array geometry. A second set of positions was produced by adding small errors to the theoretical coordinates. The total sensor positioning error, expressed as the sum of the $X$ and $Y$ displacements over all of the sensors, was $0.05 \lambda$. These "true" sensor positions represent the actual array geometry in the presence of sensor positioning errors.

The covariance matrix $w$ as formed using 1500 snapshots based on the true sensor positions. The sampling rate was approximately 8.3 samples per cycle. Two transmissions, with bearings of $0^{\circ}$ and $123^{\circ}$, were present throughout the sampling period. Each had a power of $0 \mathrm{~dB}$. The SNR was $10 \mathrm{~dB}$. This is defined to be the ratio of the power in one of the transmissions to the power of the additive noise component in the output from one of the sensors. The noise level is the same for all of the sensors and is uncorrelated between sensors.

The conventional beamforming [2], Minimum Energy [2], and MUSIC [3] techniques were compared in the presence of sensor positioning errors. Bearing spectra were generated using the following expressions.

$$
\begin{aligned}
& P_{B F}(\theta)=\underline{e}^{H}(\theta) \underline{R} \underline{e}(\theta) \\
& P_{M E}(\theta)=\frac{1}{\underline{e}^{H}(\theta) \underline{R}^{-1} \underline{e}(\theta)} \\
& P_{M U}(\theta)=\frac{1}{\underline{e}^{H}(\theta) \underline{V} \underline{V} \underline{W} \underline{e}(\theta)}
\end{aligned}
$$

where $e(\theta)$ is the signal vector for bearing $\theta^{\circ}, \underline{R}$ is the covariance matrix, and $V_{W}$ is a matrix having the noise subspace eigenvectors as its columns.

The authors are with the Department of Electrical Engineering, University of Edinburgh, The King's Buildings, Edinburgh, EH9 3JL, Scotland. 
Normally, the true sensor positions are unknown and the theoretical ones must be used instead. The effect of this substitution was investigated by generating bearing spectra using signal vectors corresponding to the theoretical sensor positions. The results are plotted in Figures 1 (a), $1(b)$, and 1(c). These should be compared with Figures 1(d), 1(c), and 1(f), which show the corresponding spectra obtained using the true sensor positions.

The conventional beamforming and Minimum Energy techniques are seen to be relatively insensitive to sensor positioning errors. The use of the theoretical positions in place of the true ones has very little effect on the bearing spectra. When the true sensor positions are used, MUSIC performs significantly better than the other two methods. However, the use of the theoretical sensor positions has a dramatic effect on the MUSIC spectrum. The peak heights are reduced considerably and there are small errors in the measured bearings.

The poor performance in the presence of sensor positioning errors is not caused by the incorrect positions per se but by the inconsistency between these positions and the theoretical ones assumed in the bearing estimation process. If the true positions could be discovered and used in place of the theoretical ones then the performance of the system would be improved.

In the proposed calibration method, the array is illuminated with transmissions from known directions and the sensor outputs are analysed to determine the true sensor positions. This "position estimation" problem is closely related to the normal bearing estimation one.

The system thus has two modes of operation. In the calibration mode, transmissions with known bearings are provided and the new calibration algorithm is used to estimate the true positions of the sensors. In the normal operational mode, the estimated sensor positions are used by a bearing estimation algorithm to measure the bearings of unknown transmissions. Since the calibration algorithm is based on MUSIC, it is recommended that MUSIC should be used to perform the bearing estimation.

\section{Calibration Algorithm}

The calibration algorithm uses triangulation to determine the true positions of the sensors. A detailed mathematical description of the technique has been published elsewhere [5]. The following discussion presents a brief outline of the method.

In Figure 2, two transmissions with bearings $\theta_{1}$ and $\theta_{2}$ are being received simultaneously. The unit vectors $z_{1}$ and $z_{2}$ point towards the transmitters. Thus the directions of propagation are $-z_{1}$ and $-z_{2}$. Each sensor position is described by a vector giving the displacement from the origin. The position of sensor $n$ is $u_{n}$. To simplify the diagram, only one sensor is shown.

During calibration, the bearings of the transmissions, $\theta_{1}$ and $\theta_{2}$, are known precisely. Thus the vectors $\underline{z}_{1}$ and $z_{2}$ are available. The aim of the calibration process is to determine the true position, $\underline{u}_{n}$, of each sensor. A transmission with a bearing of $\theta_{1}$ can only reveal the component of $\underline{u}_{n}$ in the direction of the vector $z_{1}$. In order to measure the position completely we require components in at least two different directions. The process is thus one of triangulation. Clearly, the technique is more reliable if several directions are processed rather than only two. The maximum permissible number of calibration transmissions is limited by the number of sensors in the array.

The calibration algorithm exploits the fact that the signal vectors $\underline{e}\left(\theta_{1}\right)$ and $e\left(\theta_{2}\right)$ should lie entirely in the signal subspace [3]. However, this will only be the case if the signal vectors are calculated using the true sensor positions. Signal vectors corresponding to incorrect sensor positions will generally have some component in the noise subspace.

We define a cost function for a set of sensor positions by summing the lengths of the projections of the signal vectors onto the noise subspace. The cost function can be differentiated with respect to the $X$ and $Y$ coordinate of each sensor. The calibration algorithm manipulates the sensor positions in an attempt to minimise the cost function. The process is performed iteratively using a gradient search algorithm. The iteration is initialised using the theoretical sensor positions. In practice, these are likely to be very good estimates of the true positions. 
Relative Power (dB)

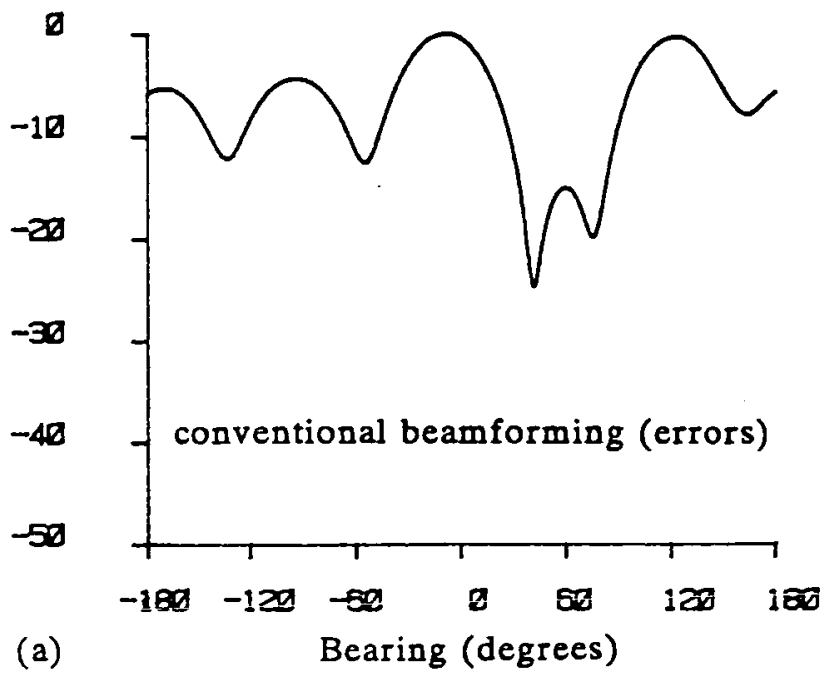

Relative Power (dB)

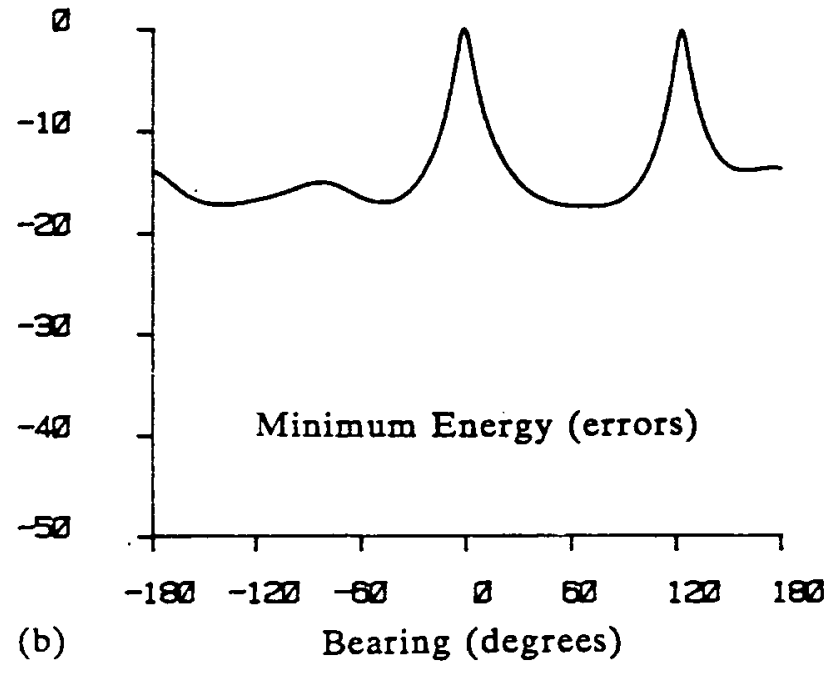

Relative Power (dB)

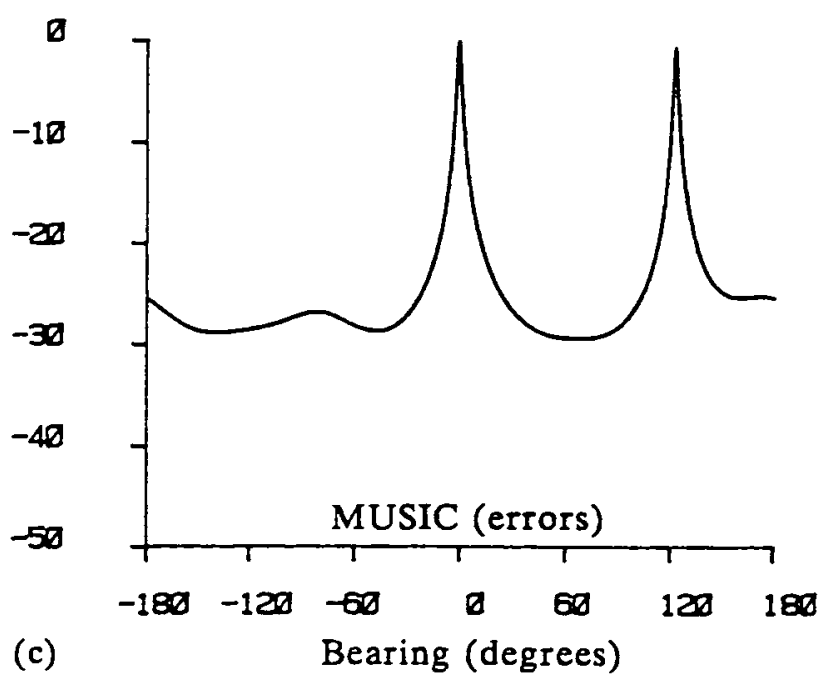

Relative Power (dB)

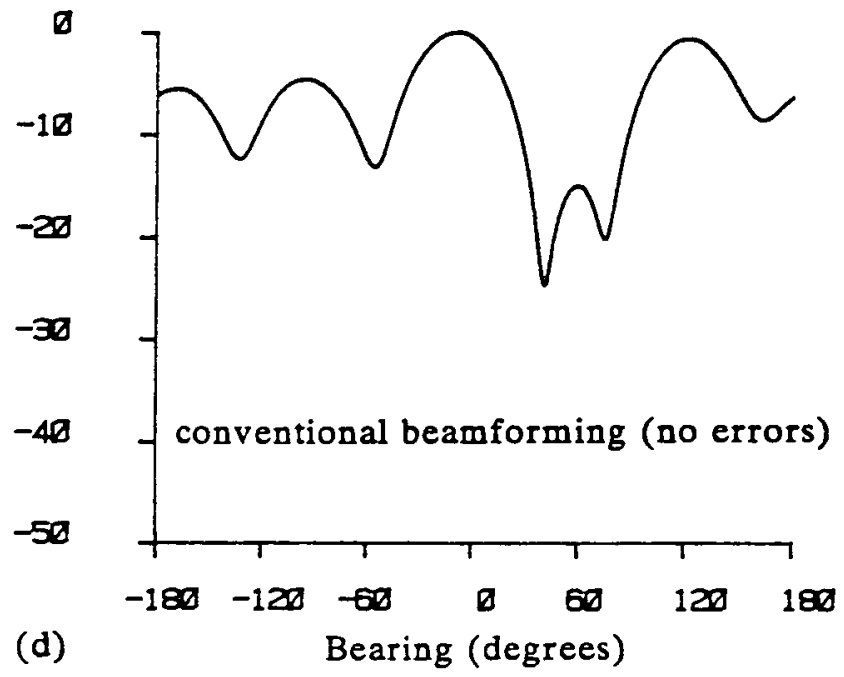

Relative Power (dB)

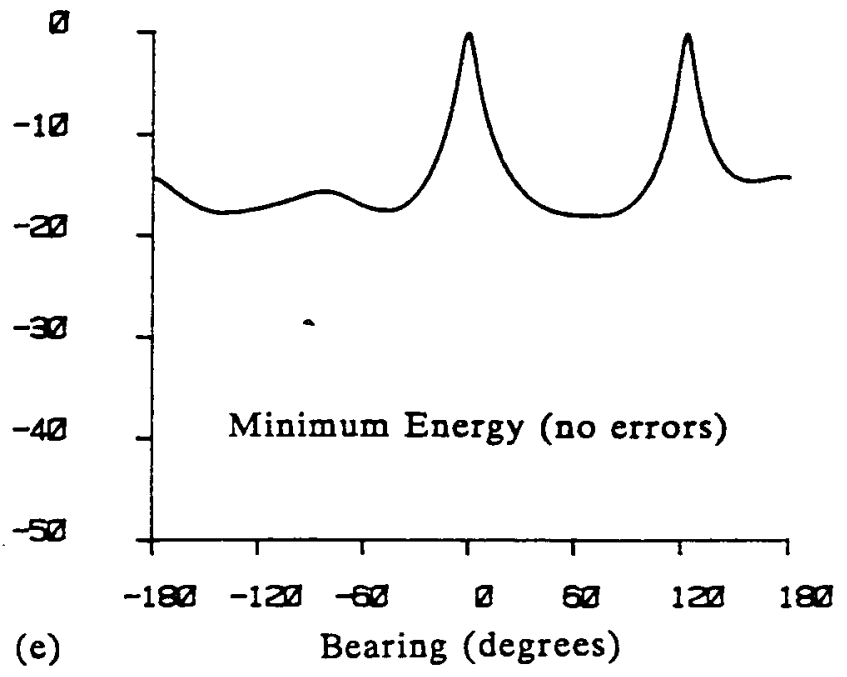

Relative Power (dB)

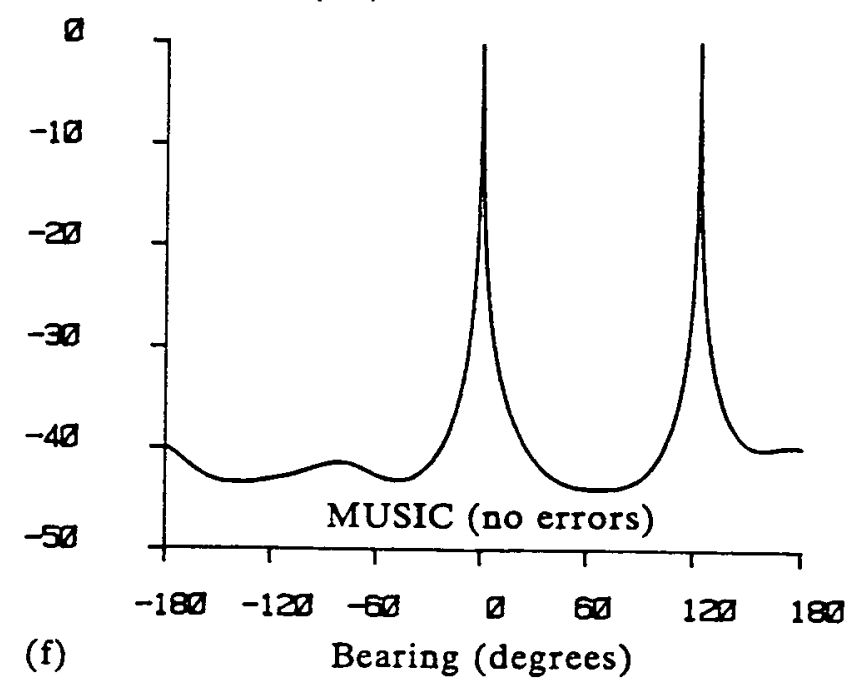




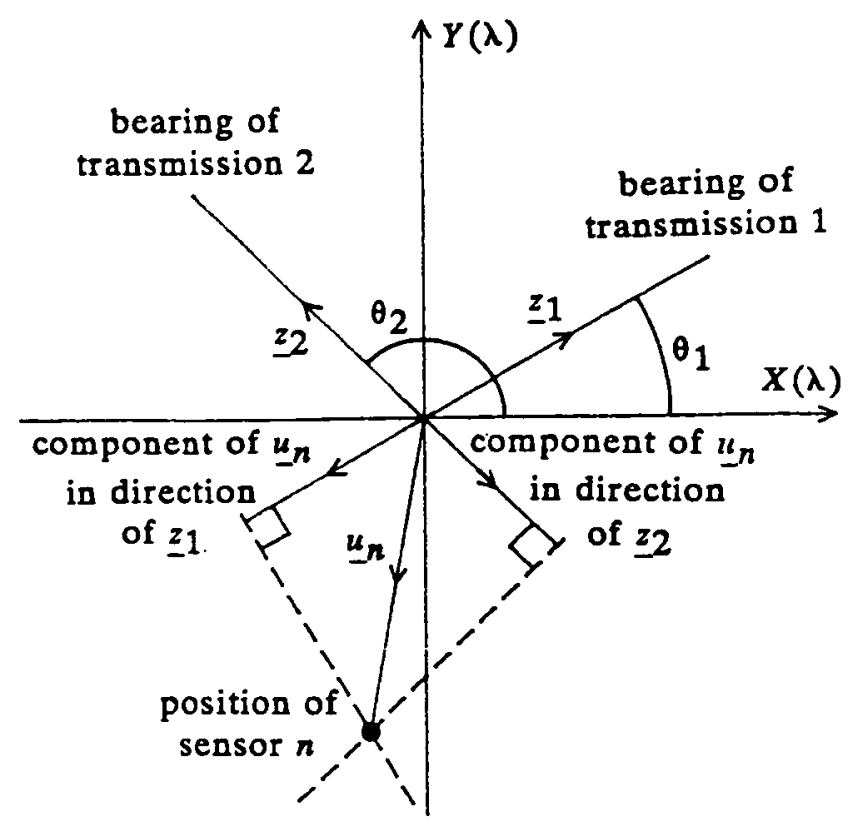

Figure 2. Triangulation

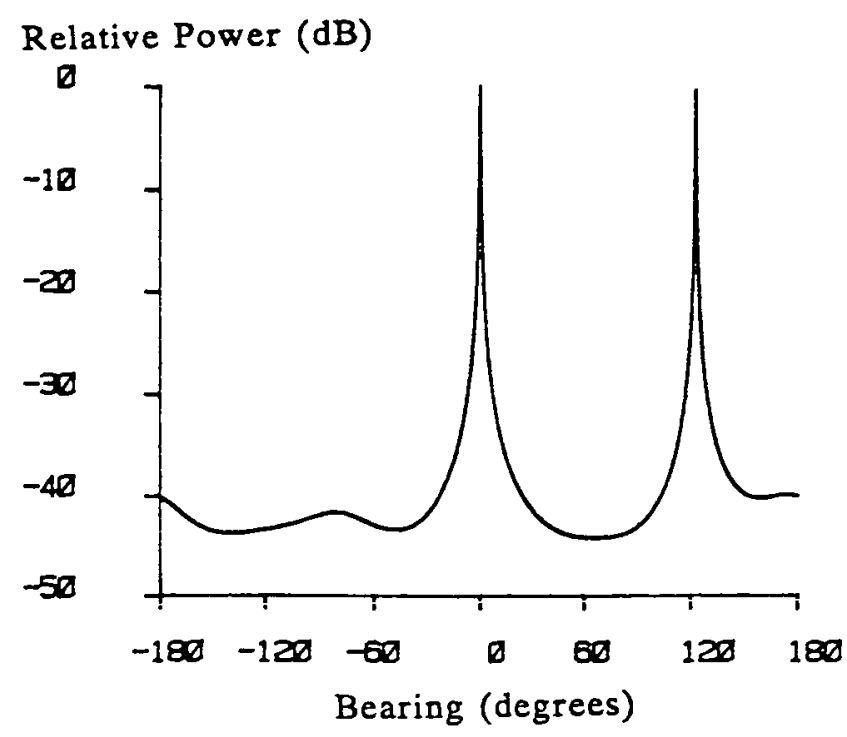

Figure 3. MUSIC with Calibration

Figure 3 shows the MUSIC spectrum obtained after calibration using the new algorithm. The array $w$ as calibrated using the signals at $0^{\circ}$ and $123^{\circ}$. The spectrum was generated using signal vectors corresponding to the estimated sensor positions produced by the calibration algorithm. The total sensor positioning error has been reduced from $0.05 \lambda$ to $0.002 \lambda$. The resulting spectrum is very similar to the one that would be produced using the true positions as shown in Figure $1(f)$. The improved performance is maintained for transmissions from other bearings.

\section{Conclusions}

The use of a calibration algorithm to compensate for sensor positioning errors in direction finding arrays has been considered. The method relies on calibration transmissions with known bearings and is related to MUSIC. Simulation results have been presented to demonstrate the improved performance of the bearing estimation process when the new algorithm is used.

\section{Acknowledgements}

The authors wish to acknowledge the sponsorship of this work by the Science \& Engineering Research Council and Racal Communications Equipment Limited.

\section{References}

[1] D.H.Johnson, "The application of spectral estimation methods to bearing estimation problems", Proc. IEEE 70, pp. 1018-1028, 1982.

[2] D.H.Johnson, S.R.DeGraff, "Improving the resolution of bearing in passive sonar arrays by eigenvalue analysis", IEEE Trans. ASSP-30, pp. 638-647, 1982.

[3] R.Schmidt, "Multiple emitter location and signal parameter estimation", Proc. RADC Spectrum Estimation Workshop, pp. 243-258, 1979.

[4] D.H.Brandwood, "Adaptive array techniques for direction finding", IEE International conference on antennas and propagation, pp. 246-250, 1985.

[5] L.P.H.K.Seymour, C.F.N.Cowan, and P.M.Grant, "Bearing estimation in the presence of sensor positioning errors", IEEE ICASSP, 1987. 


\begin{abstract}
A new calibration algorithm to compensate for sensor positioning errors in direction finding (DF) arrays is introduced. Transmissions with known bearings are analysed to determine the true sensor positions, which are subsequently used by a conventional bearing estimation algorithm to determine the angles of arrival of unknown transmissions. The proposed calibration algorithm is based on Schmidt's MUSIC method for bearing estimation. Simulation results illustrate the effects of sensor positioning errors, the improvements obtained using the new algorithm, and it robustness in the presence of other sources of error, such as uncorrelated noise and finite averaging.
\end{abstract}

\section{INTRODUCTION}

In bearing estimation, the outputs from a set of sensors are analysed to determine the bearings of signals arriving at the array $[1,2,3]$. The positions of the sensors are normally assumed to be known precisely. However, if there are errors in the positions then these assumptions are false and the bearing estimation is less accurate. Brandwood [4] presents examples of bearing spectra obtained using incorrect positions. However, no solution to the problem has been reported. This paper introduces a new algorithm to compensate for sensor positioning errors.

A comparison of the conventional beamforming [2], Minimum Energy [2], and MUSIC [3] bearing estimation techniques showed that the erross in the bearing spectrum depend on the choice of bearing estimation algorithm as well as factors such as the size of the positional errors and the bearings of the transmissions. Smail errors tend to cause a reduction in peak heights while larger ones can produce bias in the bearings as well. The algorithms have inferior resolution and detection properties in the presence of sensor positioniag errors. Unfortunately, methods such as MUSIC, which can offer excellent performance under favourable conditions, tend to be the least robust.

This effect is illustrated in Figures 1 and 2. Figure 1 shows the MUSIC spectum obtained under aormal conditions. In Figure 2, small errors have been introduced into the positions of some of the sensors. These results are described in detail below.

The poor performance in the presence of sensor positioning errors is not caused by the incorrect positions per se but by the inconsistency between these positions and the "theoretical" ones assumed in the bearing estimation process. If the true positions could be discovered and used in place of

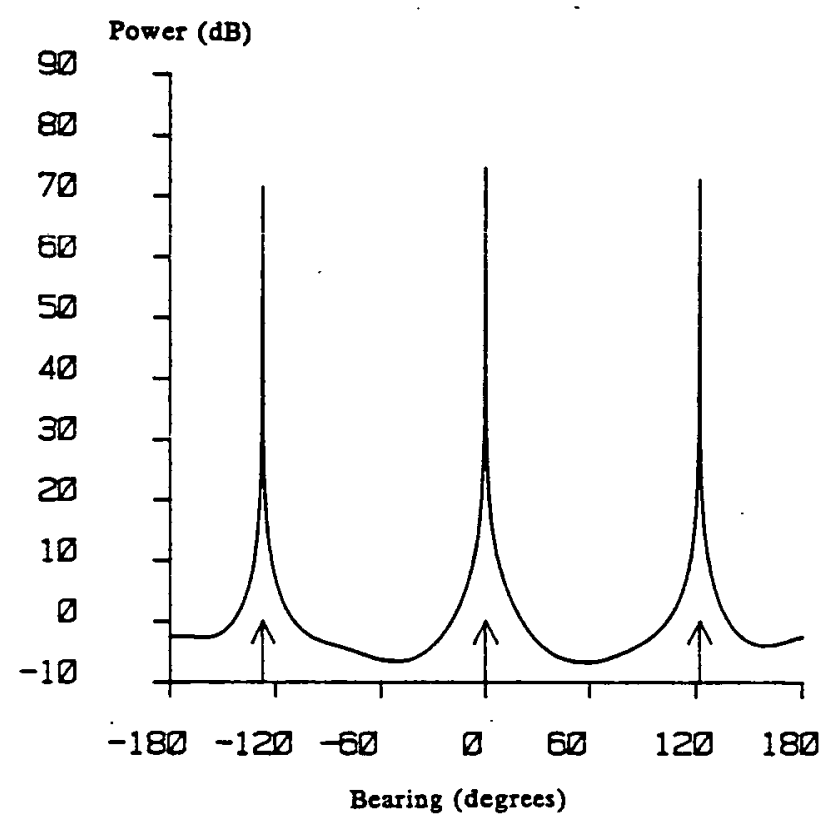

Figure 1. Bearing Spectrum using True Sensor Positions

the theoretical ones then the performance of the system would be improved.

In the proposed calibration method, the array is illuminated with transmissions from known directions and the sensor outputs are analysed to determine the true sensor positions. This "position estimation" problem is closely related to the normal bearing estimation one.

The system thus bas two modes of operation. In the calibration mode, transmissions with known bearings are provided and the new calibration algorithm is used to estimate the true positions of the sensors. In the normal operational mode, the estimated sensor positions are used by a bearing estimation algorithm to measure the bearings of unknown transmissions. Since the calibration algorithm is based on MUSIC, it is recommended that MUSIC should be used to perform the bearing estimation. 


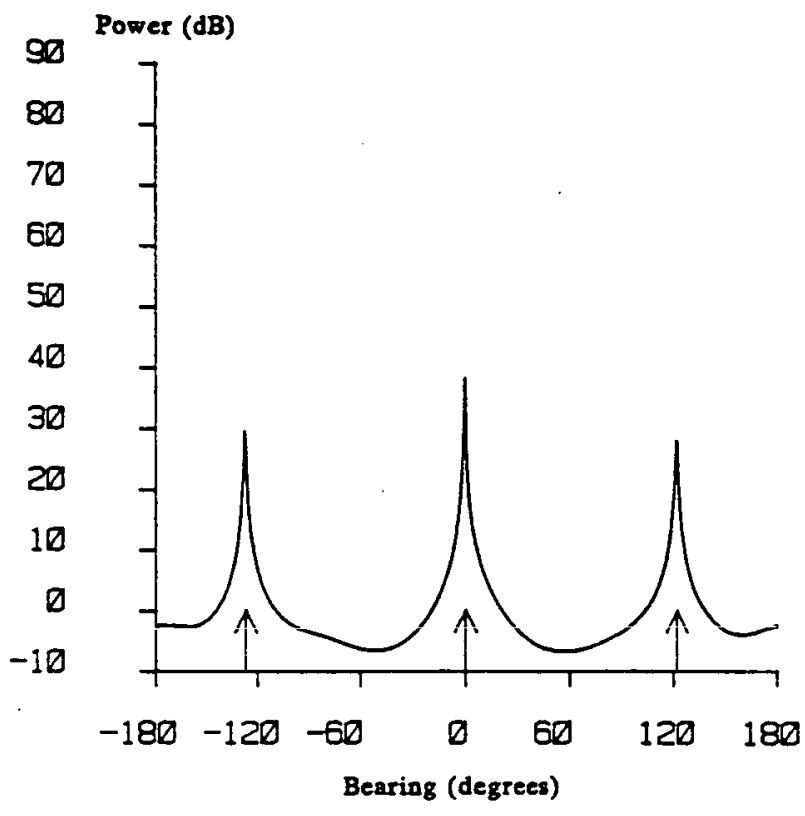

Figure 2. Bearing Spectrum using Theoretical Sensor Positions

\section{CALIBRATION ALGORITEM}

Figure 3 shows an array of $N$ sensors receiving $M$ calibration transmissions simultaneously. Each sensor position is described by a vector giving the displacement from the origin. The theoretical position of sensor $n$ is $r_{n}$ while $u_{n}$ is its true position. Additionally, we shall use $\hat{u}_{\pi}$ to denote an estimate of the true position. The array is always positioned so that sensor 1 lies at the origin.

$$
\underline{r}_{1}=\hat{u}_{1}=\underline{u}_{1}=\underline{0}
$$

The bearing of transmission $m$ is $\theta_{m}$. The unit vector $z_{m}$ points towards the transmitter. Thus the direction of propagation is $-z_{m}$. During calibration, the number of transmissions, $M$, and the bearing of each transmitter, $z_{m}$, are known precisely.

The following symbols are used to indicate the $X$ and $Y$ components of $\tau_{n}, u_{n}, \hat{u}_{n}$, and $z_{m}$.

$\tau_{\Omega}=\left[\begin{array}{c}x_{r_{n}} \\ y_{r_{n}}\end{array}\right], u_{\Omega}=\left[\begin{array}{c}x_{u_{n}} \\ y_{u_{n}}\end{array}\right], \hat{u}_{n}=\left[\begin{array}{l}\hat{x}_{u_{n}} \\ \dot{y}_{u_{n}}\end{array}\right], z_{m}=\left[\begin{array}{l}x_{z_{m}} \\ y_{z_{m}}\end{array}\right]$

The columa vectors $\hat{x}_{\mu}$ and $\hat{y}_{\mu}$ contain the $X$ and $Y$ components of the estimated sensor positions, $\hat{w}_{\lambda}$.

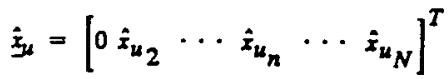

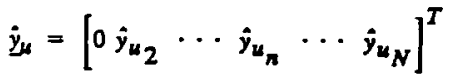

There are three possible signal vectors for transmission $m$. These correspond to the theoretical sensor positions $r_{n}$. the true ones $u_{j}$, and the estimates $\hat{u}_{R}$, and are denoted by $e_{m}, q_{m}$, and $q_{m}$ respectively. They are defined as follows.

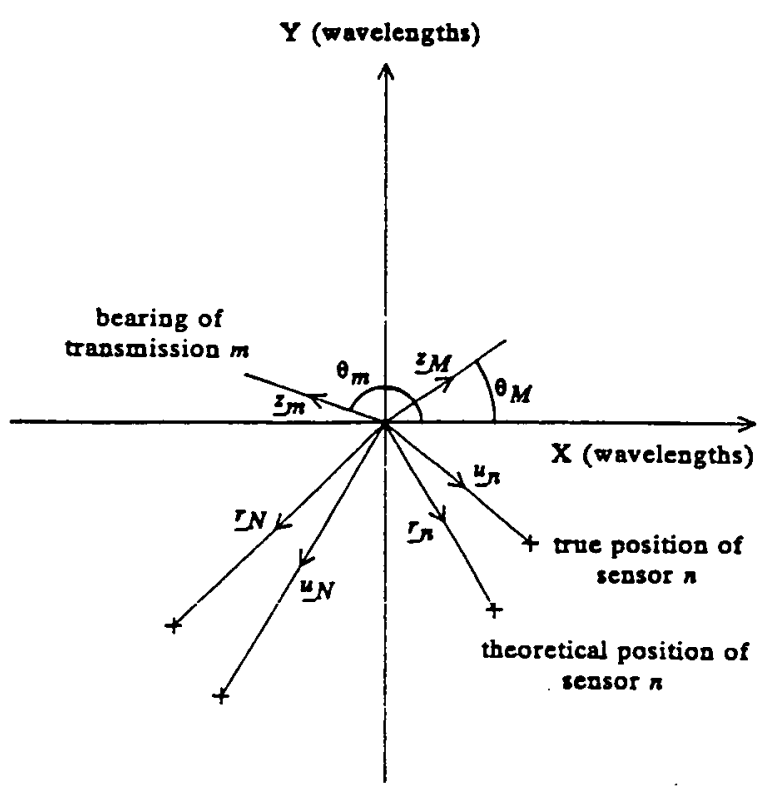

Figure 3. Sensor Positions and Transmission Bearings

$$
\begin{aligned}
& e_{m}=\left[\begin{array}{llllll}
1 & e 2 m & \cdots & e_{n m} & \cdots & e_{N m}
\end{array}\right]^{T} \\
& q_{m}=\left[\begin{array}{llllll}
1_{q_{2 m}} & \cdots & q_{n m} & \cdots & q_{N m}
\end{array}\right]^{T}
\end{aligned}
$$

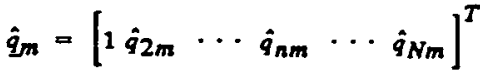

$$
\begin{aligned}
& e_{n m}=\exp \left\{\Omega \pi z_{m} \cdot r_{n}\right\} \\
& q_{n m}=\exp \left\{\Omega \pi z_{m} \cdot u_{n}\right\} \\
& \hat{q}_{n m}=\exp \left\{2 \pi z_{m} \cdot \hat{u}_{n}\right\}
\end{aligned}
$$

The matrix $\underline{V}_{w}$ bas the $N-M$ noise subspace eigenvectors as its columns.

$$
\underline{v}_{w}=\left[\begin{array}{llllll}
\underline{v}_{1} & v_{2} & \cdots & v_{h} & \cdots & v_{N-M}
\end{array}\right]
$$

where

$$
y_{h}=\left[\begin{array}{llll}
v_{1 h} & v_{2 h} & \cdots & v_{n h} \\
\cdots & v_{N h}
\end{array}\right]^{T}
$$

The length of the projection of an estimated signal vector, $\hat{q}_{m}$, onto the noise subspace is given by

$$
\left\|p_{m}\right\|^{2}=\dot{q}_{m}^{H} \underline{v}_{w} \underline{v}_{w}^{H} \dot{q}_{m}
$$

where $\boldsymbol{H}$ denotes the Hermitian transpose. 
The true signal rector, $q_{m}$, produce projections with a length of zero since they lie entirely in the tignal subspace [3].

$$
\quad\left\|p_{m} \mid\right\|^{2}=0 \text { with } \hat{q}_{n}=q_{n}
$$

However, an estimated signal vector corresponding to incorrect sensor positions will not lie entirely in the signal subspace. Therefore it must have some component in the noise subspace.

$$
|| p_{n}||^{2}>0 \text { with } \hat{q}_{n} \neq q_{n}
$$

In particular, conventional MUSIC processing assumes that the theoretical sensor positions are correct by serting $\hat{q}_{m}=\boldsymbol{\epsilon}_{m}$.

We can define a cost function for a set pf estimated sensor positions, $\hat{x}_{3 s}$ and $\dot{y}_{\mu}$, by summing ||$P_{n}||^{2}$ orer the $M$ calibration transmissions.

$$
J\left(\hat{x}_{u}, \hat{y}_{u}\right)=\sum_{m=1}^{M}|\cdot| p_{m}||^{2}
$$

The notation emphasizes that $J\left(\hat{x}_{\mu}, \hat{y}_{\mu}\right)$ is a function of the estimated sensor positions only. The transmitter bearings, $z_{n}$, are known constants. The eigenvectors, $y_{h}$, can also be regarded as constants once they have been computed.

If $J\left(\hat{x}_{\mu}, \hat{y}_{\mu}\right)=0$ then all of the estimated signal rectors, $\hat{q}_{m}$, lie entirely in the signal subspace. In this case, the estimated sensor positions, $\hat{x}_{\mu}$ and $\hat{y}_{\mu}$, producing those signal vectors are assumed to be correct provided that a number of conditions regarding the number of calibration transmissions, $M$, and their bearings, $z_{m}$, are satisfied.

From (4), if there is an error in the position of sensor $n$ then transmission $m$ can only reveal the component of that error in the direction of the vector $z_{m}$. In order to measure the error completely we require componeats in at least two different directions. The process is thus one of triangulation.

Clearly, the technique is more reliable if several directions are processed rather than only two. In the case of the calibration algorithm, the maximum permissible number of calibration transmissions is limited by the number of sensors in the array.

The algorithm manipulates the estimated sensor positions, $\dot{y}_{y}$ and $\dot{y}_{\mu}$, in an attempt to minimise the cost function, $J\left(\hat{x}_{\mu}, \hat{y}_{\mu}\right)$. The process is performed iteratively using a gradient search algorithm. The estimates at step $t$ are denoted by $\dot{x}_{u}(i)$ and $\hat{y}_{u}(l)$. The process is initialised using the theoretical sensor positions. In practice, these are likely to be very good estimates of the true positions.

$$
\dot{x}_{u_{n}}(0)=x_{r_{n}}, \quad \dot{y}_{u_{n}}(0)=y_{r_{n}}, n=1, \ldots, N
$$

At each step, the estimates are updated as follows.

$$
\begin{aligned}
& \dot{x}_{\mu}(i+1)=\dot{x}_{\mu}(i)+\mu\left[-\frac{\partial}{\partial \hat{x}_{\mu}} J\left(\hat{x}_{\mu}, \hat{y}_{\mu}\right)\right]_{\hat{x}_{\mu}=\hat{x}_{\mu}(i)} \\
& \hat{y}_{\mu}(i+1)=\hat{y}_{\mu}(i)+\mu\left[-\frac{\partial}{\partial \hat{y}_{\mu}} J\left(\hat{y}_{\mu}, \hat{y}_{\mu}\right)\right]_{\dot{y}_{\mu}=\hat{\underline{x}}_{\mu}(i)}
\end{aligned}
$$

$\mu$ is the step size in the iteration. The partial derivatives of $J\left(\hat{I}_{\mu}, \hat{y}_{\mu}\right)$ with respect to $\hat{j}_{\mu}$ and $\hat{y}_{\mu}$ are obtained analytically and are as follows.

$$
\begin{aligned}
& \frac{\partial}{\partial \hat{x}_{u}} J\left(\hat{s}_{\mu}, \hat{y}_{u}\right)=\sum_{m=1 h=1}^{M} \sum_{k=M}^{N-\mu \pi x_{m}} v_{n h} \dot{q}_{n m}^{*} \dot{q}_{m}^{H} v_{h} \\
& \left.+\Omega \pi x_{z_{m}} v_{n h} \hat{q}_{n m} v_{h}^{H} \dot{q}_{n}\right]
\end{aligned}
$$

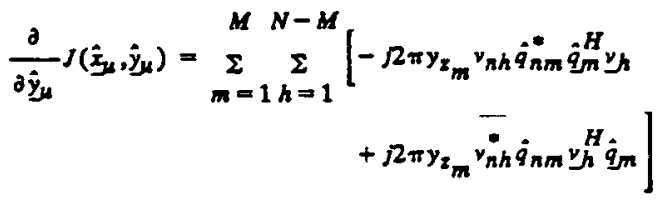

where denotes the complex conjugate.

\section{RESULTS}

\section{Scenario}

The simulation results deseribed below were obtained using an array consisting of five sensors eveny spaced around a circle of radius 0.5 wavelengths with a sixth sensor at the centre. These were the theoretical sensor positions.

SNRs of between $15 \mathrm{~dB}$ and $60 \mathrm{~dB}$ were investigated. The covariance matrix was formed using either 1600 snapshots 6400 snapshots, or analytically through mathematical expectations. When snapshot were used, the sampling rate 7 as approximately 8.21 samples per cycle.

The total sensor positioning error for a particular array was expressed as the sum of the $X$ and $Y$ displacements over all of the sensors. This measure is plotted on a logarithmic scale in the graphs.

Three calibration transmissions were present throughout the sampling period. The bearings of the transmissions were $-127,0$, and 123 degrees. Each bad a power of $0 \mathrm{~dB}$.

\section{MUSIC with Calibration.}

Figures 1, 2, and 4 illustrate the improved bearing estimation achieved by the new algorithm. The plots are all produced using MUSIC, bowever a different set of sensor positions has been used for each spectum as discussed below. The total sensor positioning error is 0.02 waveleagths. The SNR is $40 \mathrm{~dB}$ and the covariance matrix was formed using 6400 snapshots.

Figure 1 shows the spectrum obtained using the true sensor positions. This is a typical MUSIC spectrum with large, sharp peaks at the correct bearings. Clearly, this spectrum could not be obtained in practice since the true sensor positions are unknown!

The spectrum produced using the theoretical sensor positions is presented in Figure 2. This illustrates the performance that would be obtained using conventional MUSIC processing. The peak beights have been significantly reduced and there are small errors in the measured bearings.

Figure 4 shows the spectrum obtained after calibration using the new algorithm. The total sensor positioning error has been reduced from 0.02 to 0.0002 wavelengths. Since the error is so small, the resulting spectrum is very similar to the one that would be produced using the true positions as shown in Figure 1. 


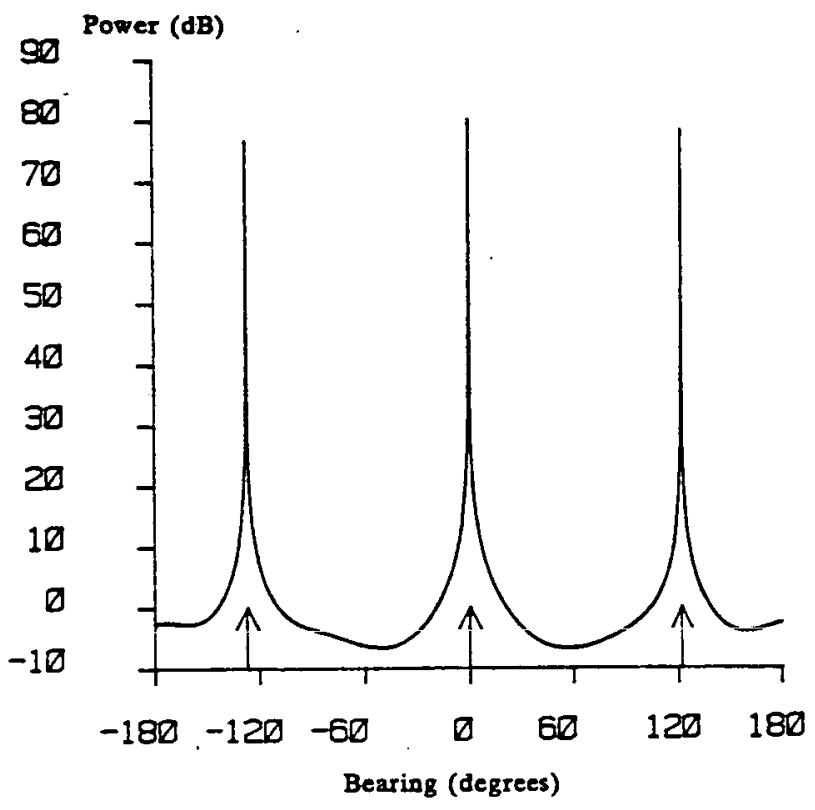

Figure 4. Bearing Spectrum using Estimated Sensor Positions

\section{Performsnce with Noise}

Figure 5 shows the relationship between the SNR and the performance of the calibration aigorithm. The SNR is defined to be the ratio of the power in one of the transmissions to the power of the additive noise component in the output from one of the sensors. The noise level is the same for all of the sensors and is uncorrelated between sensors. The vertical axis shows the total sensor positioning error after calibration. This is normalised so that zero corresponds to the original error of 0.02 wavelengths.

The three lines in Figure 5 were produced by computing the covariance matrix is different ways. When the mathematical expectation is used, the noise only affects the elements on the leading diagonal since the noise signals from different sensors are uncorreiated. A change in the SNR bas the effect of adding a constant to each of the eigenvalues. However, the eigenvectors are unaffected. Thus the performance of the calibration algorithm is indepeadent of the SNR. Note that this level of performance cannot be achieved in practice since an infinite number of snapshots would be required to approach the expectation whereas the signal eavironment is likely to be stationary only for a finite period of time.

The Figure also shows the performance obtained when the covariance matrix is formed using a relatively smail number of snapshots. Results are plotted for 1600 and 6400 snapsbots. The performance of the calibration algorithm improves with increasing SNR. The logarithmic scale reveals an approximately linear relationship.

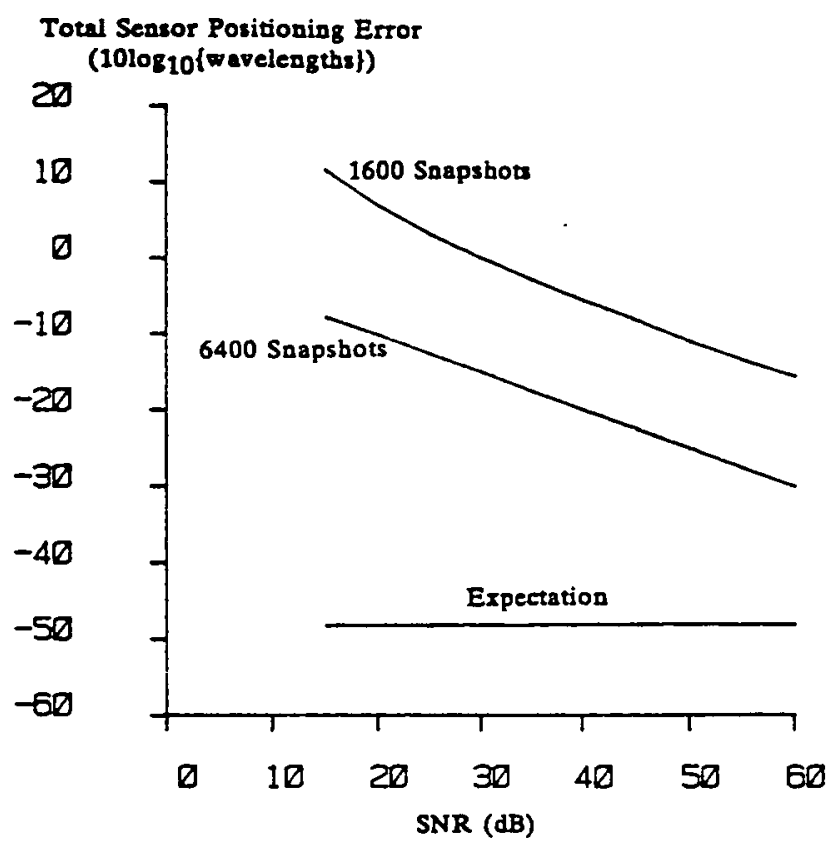

Figure 5. Performance with Noise

\section{CONCLUSIONS}

The use of a calibration algorithm to compensate for sensor positioning errors in direction finding arrays bas been considered. The method relies on calibration transmissions with known bearings and is related to Schmidt's MUSIC technique.

Simulation results have been presented to demonstrate the improved performance of the bearing estimation process when the new algorithm is used. The robustmess of the method in the presence of uncorrelated noise has also been illustrated.

\section{Acknowledgements}

The authors wish to acknowledge the sponsorship of this work by the Science Engineering Research Council and Racal Communications Equipment Limited.

\section{REFERENCES}

[1] D.H.Johnson, The application of spectral estimation methods to bearing estimation problems", Proc. IEEE 70, pp. 1018-1028, 1982.

[2] D.H.Johnson, S.R.DeGraff, "Improving the resolution of bearing in passive sonar arrays by eigenvalue analysis", IEEE Trans. ASSP-30, Pp. 638-647, 1982.

[3] R.Schmidt, "Multiple emitier location and signal parameter estimation", Proc. RADC Spectrum Estimation Workshop, pp. 243-258, 1979.

[4] D.H.Brandwood, -Adaptive array techniques for direction finding", IEE International conference on antennas and propagation, pp. 246-250, 1985. 